\title{
WATER QUALITY OF SOMERVILLE LAKE SOUTH-CENTRAL TEXAS
}

By Emma McPherson and H. B. Mendieta

U.S. GEOLOGICAL SURVEY

Water-Resources Investigations Report 82-4124

Prepared in cooperation with the U.S. ARMY CORPS OF ENGINNERS

Austin, Texas

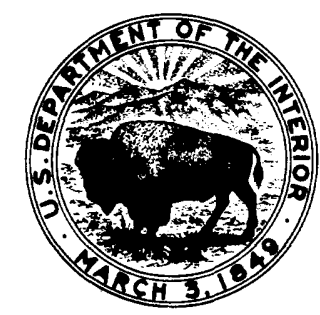




\section{UNITED STATES DEPARTMENT OF THE INTERIOR}

JAMES G. WATT, Sec retary

GEOLOGICAL SURVEY

Dallas L. Peck, Director

For additional information

write to:

District Chief

U.S. Geological Survey

649 Federal Building

300 E. Eighth Street

Austin, TX 78701
For sale by:

Open-File Services Section Western Distribution Branch U.S. Geological Survey Box 25425, Federal Center Lakewood, C0 80225 


\section{CONTENTS}

Page

Abstract-1-

Introduction-

Purpose of this report-and 2

Description of Somerville Lake and its environment-_.

Hydrologic data-

St reamflow records-10 6

Water quality of Somerville Lake-1 9

Thermal stratification- 9

Dissolved oxygen-1. 12

Dissolved iron and dissolved manganese-1

Total inorganic nitrogen and total phosphorus-_. 17

Dissolved solids, dissolved chloride, dissolved sulfate, and hardness-..... 29

Water transparency-1. 29

Phytoplankton-... 32

Summary-1-_-_- 32

Selected references 


\section{ILLUSTRATIONS}

Page

Figure 1. Map showing locations of water-quality data-collection

sites on Somerville Lake--..- 3

2. Map showing location of the Somerville Lake watershed

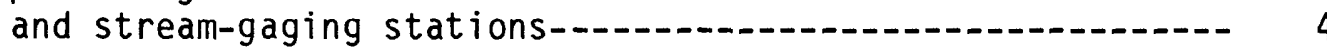

3-20. Graphs showing:

3. Monthly mean water discharges for Middle Yegua Creek near Dime Box, October 1971-September 1980

4. Monthly mean water discharges for East Yegua Creek near Dime Box, October 1971-September 1980

5. Monthly mean water discharges for Yegua Creek near Somerville, October 1971-September 1980

6. Variations in monthly mean air temperatures at Somerville Dam and water temperatures at sites $A_{C}$ and $F_{C}$ during

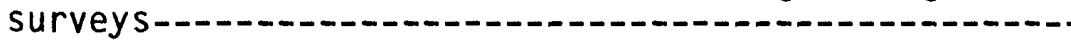

7. Seasonal profiles of water temperatures and dissolved oxygen for site Ac--

8. Variations in average concentrations of dissolved oxygen during summer and winter surveys....-..........-

9. Seasonal profiles of dissolved oxygen, dissolved iron, and dissolved manganese for site $A_{1}$

10. Variations in average concentrations of dissolved iron during summer and winter surveys-

11. Variations in average concentrations of dissolved manganese during summer and winter surveys-.....

12. Variations in concentrations of dissolved iron and dissolved manganese at site $A_{C}$, August 1975-August 1980---

13. Variations in average concentrations of total inorganic nitrogen during summer and winter surveys-_..-

14. Variations in average concentrations of total

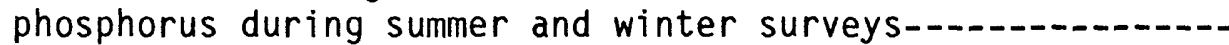

15. Seasonal profiles of water temperatures, total inorganic nitrogen, and total phosphorus for site Ac-

16. Seasonal profiles of water temperatures, total inorganic nitrogen, and total phosphorus for site $\mathrm{F}_{\mathrm{C}}$

17. Variations in concentrations of total inorganic nitrogen and total phosphorus at site AC, August 1975August 1980

18. Variations in concentrations of total inorganic nitrogen and total phosphorus at site $F_{C}$, August 1975August 1980

19. Variations in volume-weighted average concentrations of dissolved solids, dissolved chloride, dissolved sulfate, and hardness and in contents for Somerville Lake, August 1975-August 1980

20. Variations in average concentration of dissolved solids during summer and winter surveys-... 


\section{TABLES}

Page

Table 1. Chemical and physical characteristics of water from

Yegua Creek near Somerville (station 08110000),

October 1968-September 1980-1 36

2-17. Chemical-quality surveys of Somerville Lake:

2. August 29, 1975

3. February 3, $1976 \ldots$

4. April 29, 1976-_._. 43

5. August 20, 1976- 47

6. February 16, $1977 \ldots \ldots$

7. May 27, $1977 \ldots 5$

8. September $30,1977 \ldots \ldots$

9. March 13, 1978 61

10. July 14, 1978-_. 64

11. October 11, 1978 67

12. January 9, 1979_._. 70

13. June 19, 1979 73

14. August 3, 1979

15. January 30, $1980 \ldots$

16. May 21, 1980

17. August 29, 1980 
METRIC CONVERSIONS

Factors for converting inch-pound units to metric equivalents are given in the following table:

\begin{tabular}{|c|c|c|}
\hline From & Multiply by & To obtain \\
\hline acre & 4,047 & square meter \\
\hline acre-foot & 1,233 & cubic meter \\
\hline cubic foot per second $\left(\mathrm{ft}^{3} / \mathrm{s}\right)$ & .02832 & cubic meter per second \\
\hline foot & .3048 & meter $(\mathrm{m})$ \\
\hline mile & 1.609 & kilometer \\
\hline square mile & 2.590 & square kilometer \\
\hline ton & .9072 & tonne \\
\hline
\end{tabular}

National Geodetic Vertical Datum of 1929 (NGVD of 1929): A geodetic datum derived from a general adjustment of the first-order level nets of both the United States and Canada, formerly called "mean sea level." 


\title{
WATER QUALITY OF SOMERVILLE LAKE
}

SOUTH-CENTRAL TEXAS

By

Emma McPherson and H. B. Mendieta

\begin{abstract}
Somerville Lake in south-central Texas is a shallow lake, with a mean depth of 14 feet. The maximum depth of the submerged channel of Yegua Creek is usually less than 35 feet and in most areas of the lake the depth is 1 es than 10 feet.

Several factors including thermal circulation resulting from the cooling of surface water, wind action, and the large inflow volume in realtion to the lake vol ume combine to keep the lake well mixed throughout the year. The oxygen concentrations remain high areally and at depth because of good circulation of lake waters during most of the year. Even in summer most bottom oxygen concentrations were in excess of 50 percent of saturation.

Due to year-round high percent oxygen saturation from surface to bottom in most parts of the lake, caused by the frequent periods of circulation that occur during all seasons, concentrations of dissolved iron, and manganese reamin 10w. Dissovled iron concentrations were less than 50 micrograms per liter and dissolved manganese concentrations were less than 40 micrograms per liter. The total inorganic nitrogen concentrations varied little throughout the 1 ake. During the summer, concentrations were 0.01 milligram per 1 iter at the surface to 0.02 milligram per liter at the bottom; and during the winter 0.11 milligram per liter at the surface and 0.10 milligram per liter at the bottom. Concentrations in the headwaters were about double those in the lake. Surface and bottom total phosphorus concentrations, during summer and winter averaged about the same, 0.04 and 0.06 milligram per 1 iter throughout the lake, except in the headwaters where the concentrations were about double those in the lake.
\end{abstract}

Homogeneous or near homogeneous concentrations of total phosphorus and inorganic nitrogen can occur at any time of the year throughout the lake. Total phosphorus concentrations did not increase during the year or during the study period. On the other hand total inorganic nitrogen concentrations did show an annual cycle and were highest in the spring and lowest in late summer or fall. During periods of large releases of water, the more sol uble total inorganic nitrogen was flushed from the lake.

The concentration of dissolved solids ranged from 139 to 292 milligrams per liter and averaged about 220 milligrams per liter. Dissolved chloride concentrations ranged from 20 to 68 milligrams per liter and averaged 43 mill igrams per liter. Di ssolved sulfate concentrations ranged from 30 to 130 milligrams per liter and averaged 63 milligrams per liter. The total hardness of 
the water ranged from 75 to 140 milligrams per 1 iter, expressed as calcium carbonate, placing it in the moderately hard to hard (61 to 180 milligranis per liter) classification. The concentrations of principal dissolved constituents indicate that Somerville Lake is an excellent source of water for municipal, industrial, or agricultural use.

\section{INTRODUCTION}

The U.S. Geological Survey periodically has made comprehensive waterqual ity surveys of selected lakes and reservoirs in Texas since October 1961 as part of a continuing cooperative program with State, Federal and 1ocal agencies to inventory the surface-water resources of the State. During the 1975 water year, the program was expanded in cooperation with the U.S. Army Corps of Engineers to include more lakes for the number of years required to define a range of hydrologic conditions. Water-quality surveys during the spring, summer, and winter on Somerville Lake included onsite measurements of dissolved oxygen, specific conductance, ph, and temperature. Water samples also were collected and analyzed for dissolved chemical constituents and nutrients.

\section{Purpose of This Report}

This report summarizes the variations of selected water-quality constituents and properties for Somerville Lake based on water-qual ity records collected from August 1975 to August 1980. The report al so contains information on the watershed environment in relation to the quality of water in the impoundment.

\section{Description of Somerville Lake and Its Environment}

Somerville Dam, at the southwest edge of the city limits of Somerville in south-central Texas, is on Yegua Creek 20.0 miles upstream from its junction with the Brazos River. The impoundment formed by the dam is Somerville Lake, which is located in the south-central part of Burleson County and the northcentral part of Washington County, with the upper reaches extending into Lee County. The submerged channel of Yegua Creek is the boundary between Burleson and Washington Counties, dividing the main body of the lake surface almost equally between the two counties. In the upstream part of the lake, Yegua Creek al so forms the boundary between Burleson and Lee Counties. The largest part of the watershed is in Lee County, but only a small part of the 1 ake 1 ies in that county (figs. 1 and 2).

Yegua Creek, one of the principal tributaries of the Brazos River, is formed by the confl uence of the East and Middle Yegua Creeks at a point about 14 miles west of Somerville. The Yegua Creek watershed is about 62 miles 1 ong and 32 miles wide and has a drainage area of 1,320 square miles. About 76 percent of this drainage area is upstream from Somerville Dam (fig. 2). 


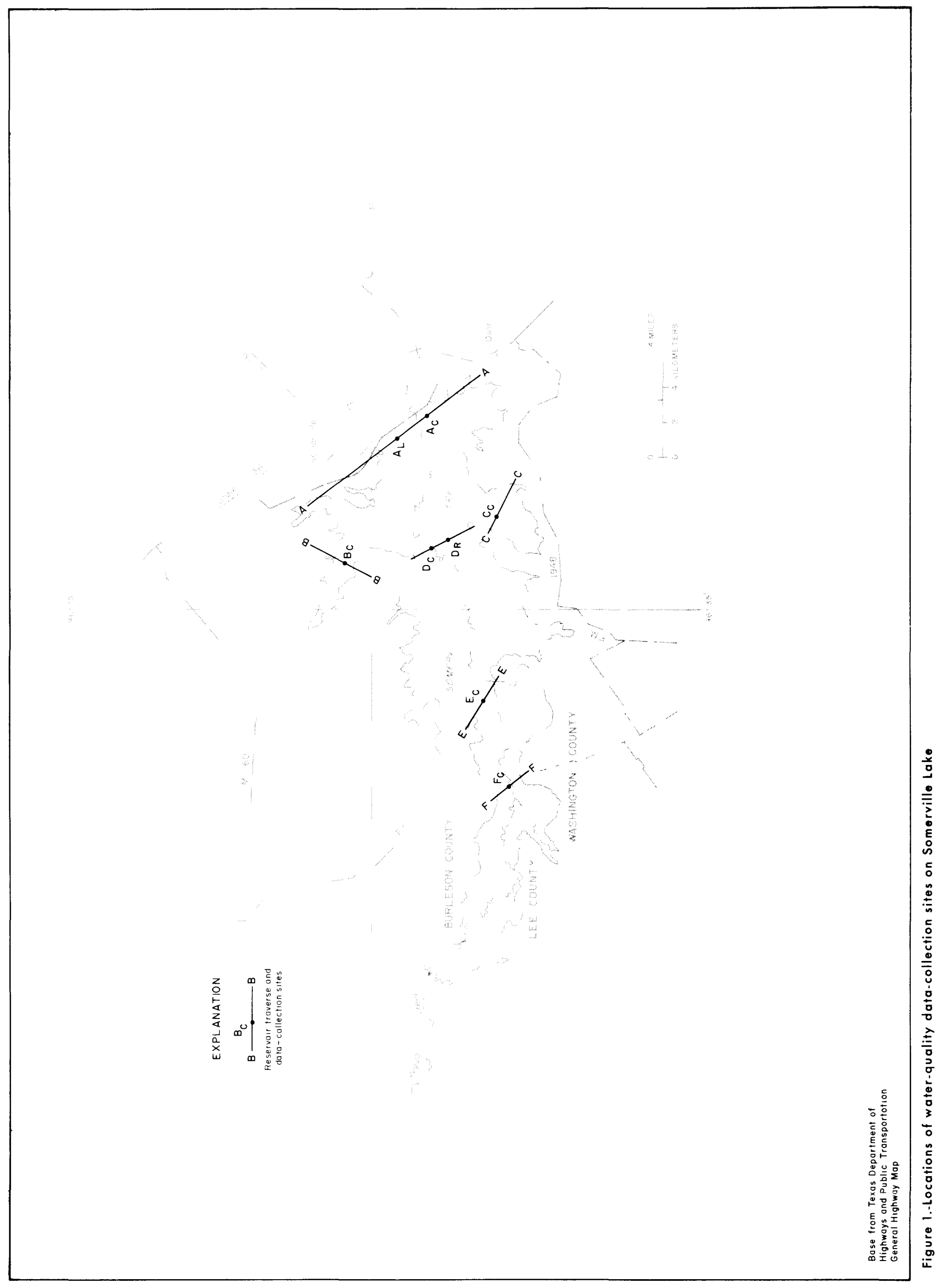




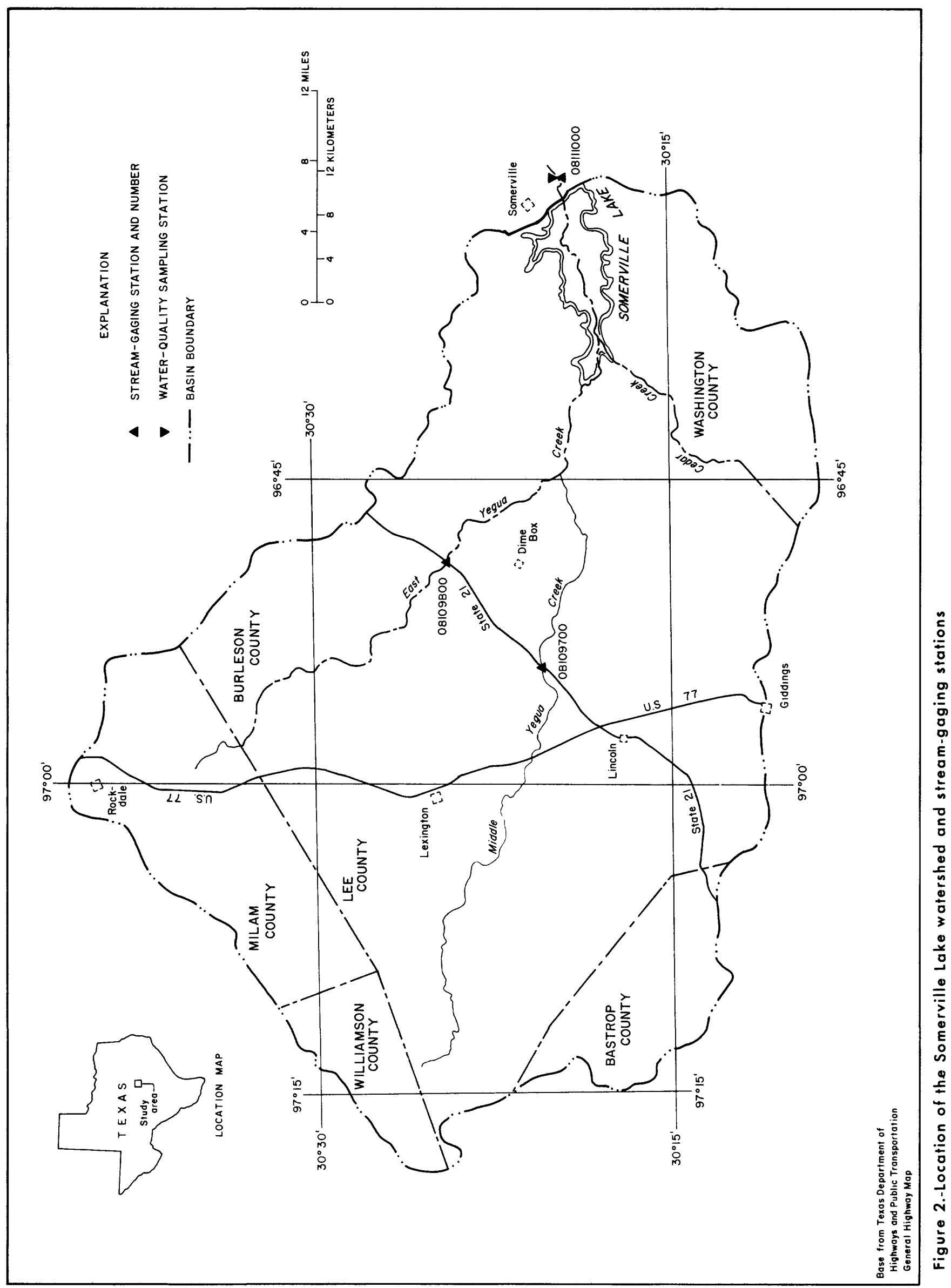


Somerville Lake was constructed and is operated by the Corps of Engineers for flood control, conservation storage, and other beneficial uses for the surrounding area. Construction of Somerville Dam began June 4, 1962, and was completed on October 27, 1967. The following data regarding the dam and lake were compiled by Dowell and Petty (1973, p. 12-29.0-A):

\begin{tabular}{lcrr}
\hline \multicolumn{1}{c}{ Feature } & $\begin{array}{c}\text { El evation } \\
\text { (feet above } \\
\text { NGVD of 1929) }\end{array}$ & $\begin{array}{c}\text { Capacity } \\
\text { (acre-feet) }\end{array}$ & $\begin{array}{c}\text { Surface } \\
\text { area } \\
\text { (acres) }\end{array}$ \\
\hline Top of dam & 280.0 & -- & -- \\
Maximum design water surface & 274.5 & $1,028,800$ & 39,800 \\
Spillway crest & 258.0 & 507,500 & 24,400 \\
Top of conservation storage space & 238.0 & 160,100 & 11,460 \\
Maximum tailwater & 243.8 & -- & -- \\
Streambed & 200.0 & 0 & 0 \\
Sediment reserve below & 238.0 & 16,200 & -- \\
Sediment reserve above & 238.0 & 9,700 & -- \\
Usable conservation storage space & -- & 143,900 & -- \\
\hline
\end{tabular}

Del iberate impoundment of water began on January 3, 1967. The conservation storage space reached its capacity for the first time on May 11, 1968. Somerville Lake is a shallow lake with a mean depth of 14 feet. The depth in the main channel usually is less than 35 feet, and in most other areas of the lake, depths less than 10 feet.

The lake as well as the entire drainage area of 1,007 square miles upstream from the dam is located on gently rolling terrain in the south-central section of Texas, which is part of the West Gulf Coastal Plain (Fenneman, 1938, p. 102). Locally the area is recognized as the Post Oak Belt, the western part of the East Texas Timber Belt. The land surface rises from the southeast to the northwest, ranging from 200 feet above sea level on the streambed at the dam to 760 feet above sea level in the Yegua Knobs area, 12 miles west-southwest of Lexington.

The soils of the drainage area are mostly sandy loams with smaller amounts of clay loams and gravelly clay loams. The soils are weathered Tertiary deposits. Several physiographic features trend northeastward to southwestward across the drainage area in conformance with the geological outcrops.

The geologic units range from the oldest in the west to the youngest in the east. All are of the Eocene Series except the youngest formation which is Miocene. The Wilcox Group, undifferentiated, crops out in the headwaters of 
the East, Middle, and West Yegua Creeks. Eastward, seven outcrops of the Claiborne Group follow in sequence: the Carrizo Sand, the Reklaw Formation, the Queen City Sand, the Weches Greensand, the Sparta Sand, the Cook Mountain Formation, and the Yegua Formation. The Jackson Group, undifferentiated, crops out parallel to both sides of the main stem of Yegua Creek. The Catahoula Sandstone, which borders the eastern boundary of the watershed, is the last and youngest unit in the sequence.

Development in the watershed is predominantly rural. The loamy, gently rolling terrain is used mostly by small family farms and stock ranches. About two-thirds of the area is used for cropland or improved pasture. Cattle, hogs, and poultry lead in livestock production. Peanuts, which grow well in sandy loams, are the chief cash crop. Wheat, oats, cotton, corn, grain sorghum, hay and watermelons al so contribute to the economy of the area.

There are only three centers of population within the watershed. Giddings (population 12,000) lies on the southern boundary of the watershed while almost all of Rockdale (population 5,611) is within the northern boundary. Lexington (population 1,065) lies in the central part of the watershed.

In the northwest part of the watershed, near Rockdale, the Alcoa al uminum plant has been strip mining about 2 million tons of 1 ignite per year since 1952 for its powerplant fuel (W. E. Michalke, Al uminum Company of America, oral commun., 1981). Lignite has been mined intermittently at this site since the turn of the century. Mining was continuous from 1920 to 1940, when cheap natural gas became available.

Since 1975, oil devel opment in the Austin Group, which underlies the study area, has made a drastic impact on the economy of the area. The environmental changes now taking place may result in significant changes in the qual ity of water draining into Somerville Lake in the future. The number of producing oil wells increased from 1 in 1974 to 150 by the end of 1978 . More than 2,500 wells were drilled between 1978-81. In 1981 there were 120 drilling rigs operating in the "Giddings trend," most of which lies within the Somerville Lake watershed. As a result of the oil boom, the population of the city of Giddings has grown from 3,200 in 1975 to an estimate of more than 12,000 in 1981. Thousands of oilfield workers, who live in trailers and follow the drilling rigs, are al so dispersed in the Yegua watershed ( $\mathrm{J}$. Socha, Giddings Chamber of Commerce, oral commun., 1981).

\section{HYDROLOGIC DATA}

\section{Streamfl ow Records}

Daily streamflow stations on Middle Yegua Creek near Dime Box (station 08109700) at mile 17.5 and on East Yegua Creek near Dime Box (station 08109800) at mile 12.2 have been operated by the Geological Survey since August 1962. Mean-annual discharges for these stations for $1962-80$ were $53.7 \mathrm{ft} 3 / \mathrm{s}$ for Middle Yegua Creek near Dime Box (fig. 3) and $59.9 \mathrm{ft} / \mathrm{s}$ for East Yegua Creek near Dime Box (fig. 4). No water-qual ity data have been collected at these stations. A streamflow station on Yegua Creek near Somerville (station 08110000) 1.0 mile downstream from Somerville Dam has been in operation since May 1924. 


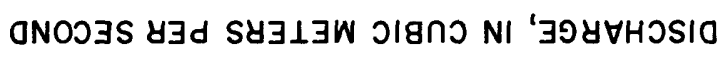

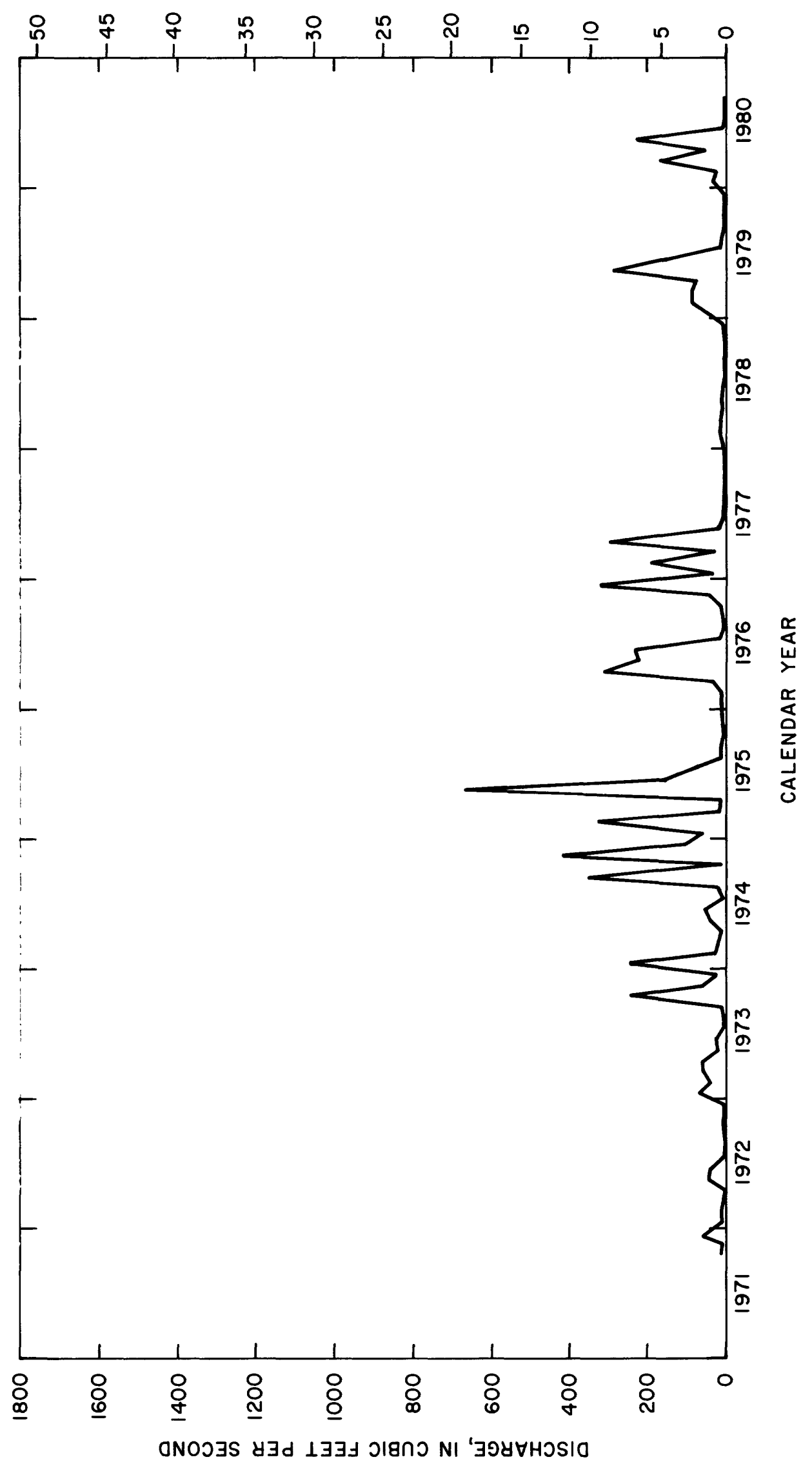

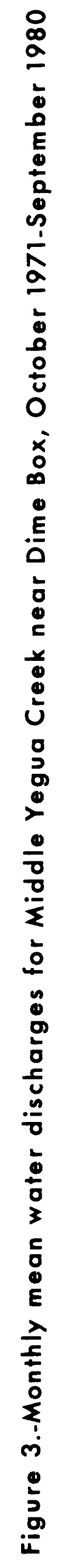




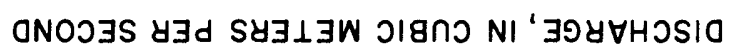

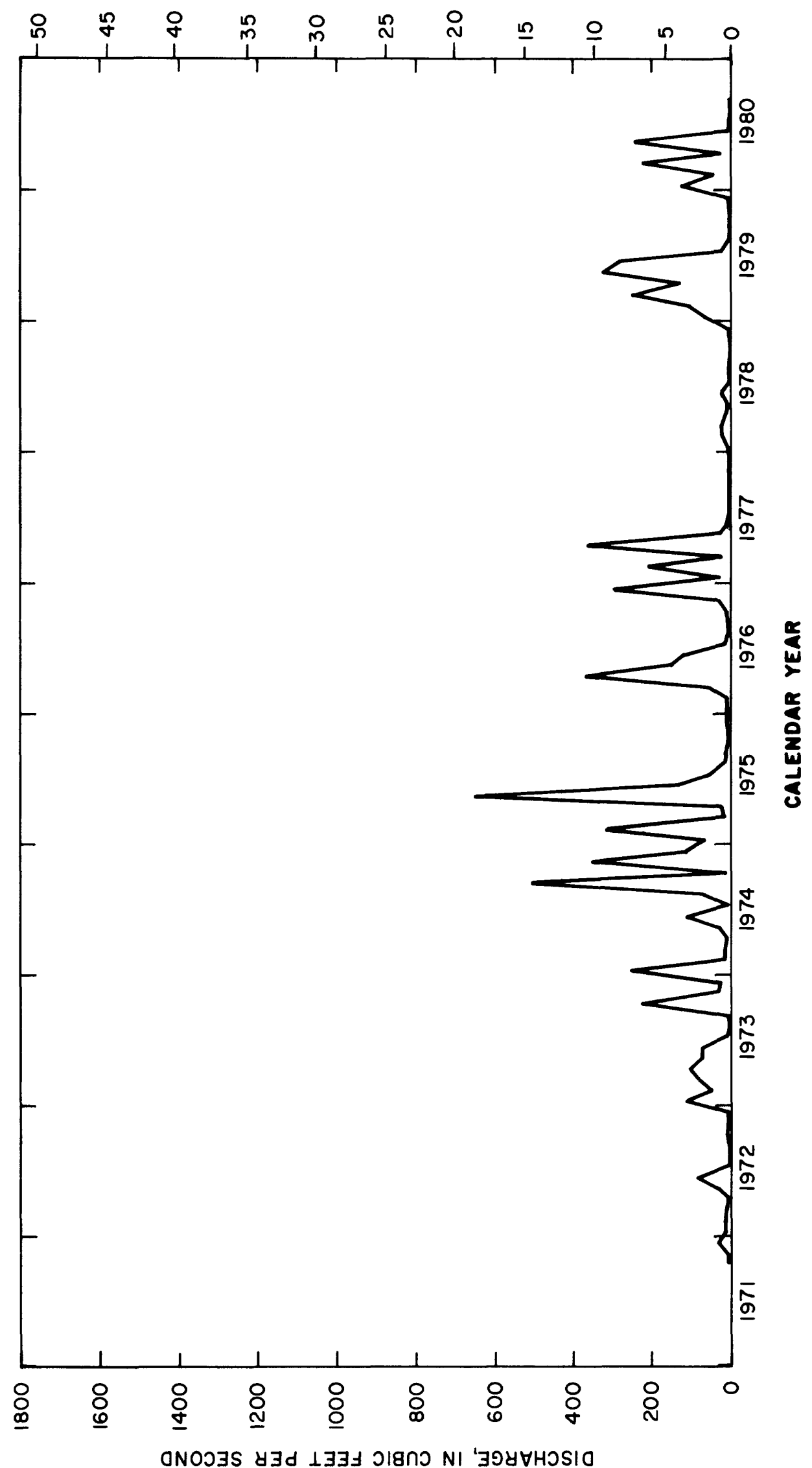

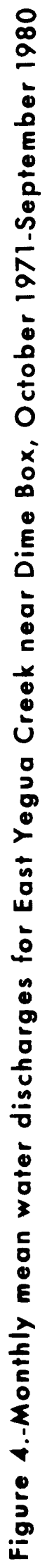


Unregulated mean-annual discharge for 1924-65 was $290 \mathrm{ft}^{3} / \mathrm{s}$. Regulated meanannual discharge for $1965-80$ was $314 \mathrm{ft} / \mathrm{s}$ (fig. 5). Water-qual ity data for this station have been collected from September 1961 to September 1967 and from October 1968 to the present and table 1 shows a statistical summary.

From October 1971 through September 1980, the combined discharge at the two upstream streamflow stations was about 38 percent of the discharge at Yegua Creek near Somerville. The mean-annual discharge at Yegua Creek near Somerville is greater than the combined flow of the Salt and Double Mountain Forks of the upper Brazos River watershed, both tributaries having much larger drainage areas. Streamflow and water-qual ity data collected by the Geol ogical Survey are publ ished annually in the Geological Survey series, "Water Resources Data for Texas."

Somerville Lake was proposed and construction started with minimal waterquality data available. In "Chemical Qual ity of Surface Waters in the Brazos River basin in Texas" (Irel an and Mendieta, 1964), the following statement was made about Yegua Creek: "The quality-of-water record for this stream consists of the analysis of only two samples--one in 1942 and another in 1959. Both of these samples were taken at low flow, and the water was of only fair quality. Much better water could be expected during high flows, and water stored in a reservoir on Yegua Creek probably would contain less than $500 \mathrm{mg} / \mathrm{L}$ (milligrams per liter) of dissolved solids. Water in Yegua Creek should be similar to that of the Navasota River, whose drainage area is directly across the Brazos, and is underlain by similar rocks. Available chemical-qual ity data indicate that all streams draining the belt of Tertiary rocks, which extends along the Texas gulf coast, have produced water of good quality." At that time U.S. Public Health Service "Drinking Water Standards" (1962) specified that the total dissolved solids should not exceed $500 \mathrm{mg} / \mathrm{L}$ if more suitable supplies were available.

The average total dissolved sol ids concentration for Somerville Lake during the $1975-80$ study was $220 \mathrm{mg} / \mathrm{L}$. This compares favorably with an average total dissolved solids concentration of $278 \mathrm{mg} / \mathrm{L}$ for Na vasota River near Bryan during the 1959-60 records that were available at the time of the water-quality projection. The conservative "less than $500 \mathrm{mg} / \mathrm{L}$ of dissolved sol ids" statement was used because that was the criteria set for a public supply.

\section{Water Qual ity of Lake Somerville}

\section{Thermal Stratification}

Impoundment of water in a lake or reservoir may result in significant changes in water quality. Some of the changes may be beneficial; other changes may be detrimental. Many of the detrimental changes can be related to thermal stratification--layering of the water due to temperatureinduced density differences. 
anOJ

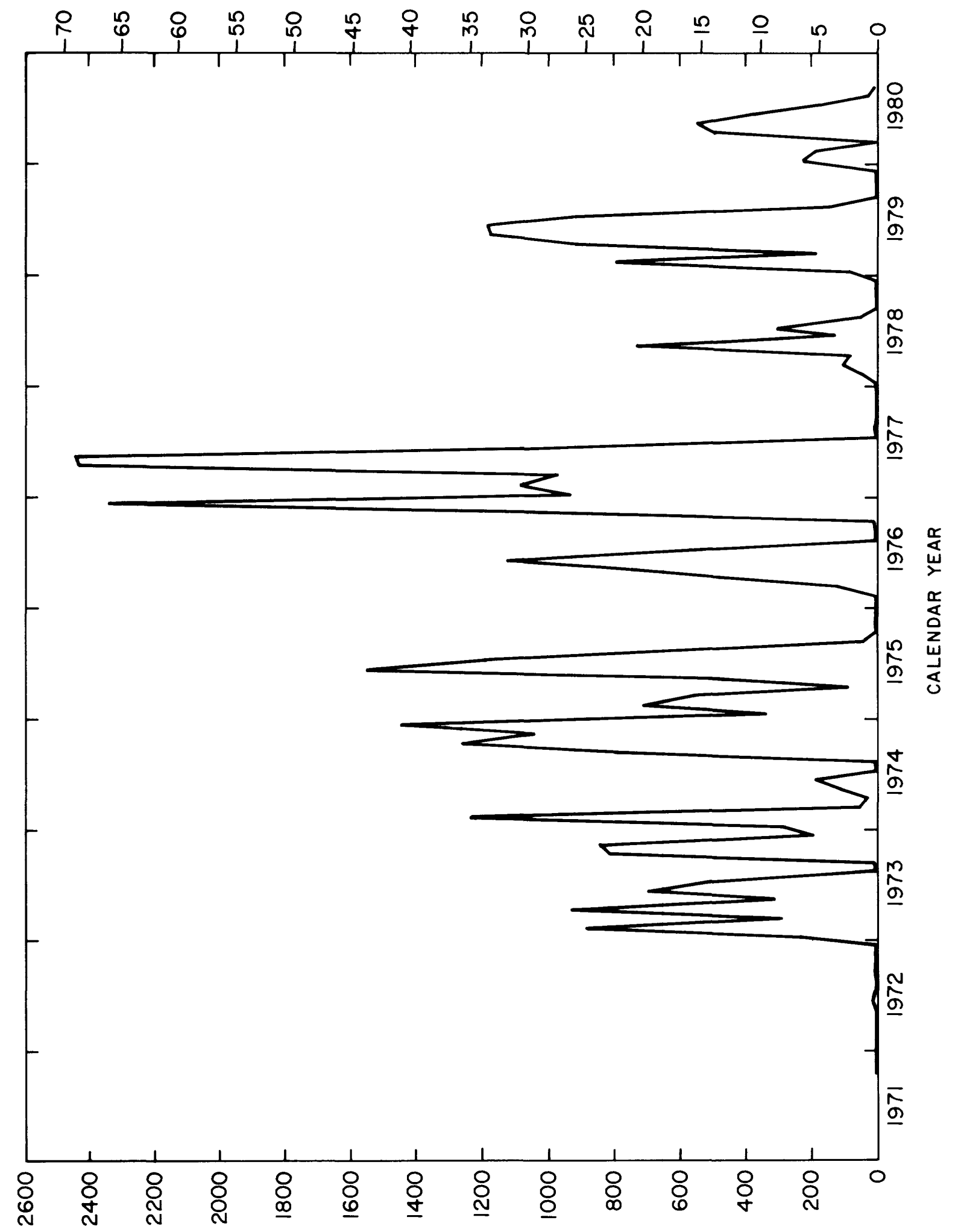

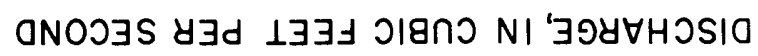


The following table (Weast, 1975, p. F-5) shows that pure water reaches its maximum density at a temperature of about $4^{\circ} \mathrm{C}$ (degrees Cel sius) and that the difference in density per $1^{\circ} \mathrm{C}$ is much greater at warmer temperatures than at cooler temperatures.

\begin{tabular}{cc}
\hline $\begin{array}{c}\text { Temperature } \\
\text { (degrees } \\
\text { Cel sius) }\end{array}$ & $\begin{array}{c}\text { Density } \\
\text { (grams per } \\
\text { milliliter) }\end{array}$ \\
\hline 0.0 & 0.999868 \\
4.0 & 1.000000 \\
5.0 & .999992 \\
10.0 & .999728 \\
15.0 & .999129 \\
20.0 & .998234 \\
25.0 & .997075 \\
30.0 & .995678 \\
35.0 & .994063 \\
\hline
\end{tabular}

For example, a change in temperature from $29^{\circ}$ to $30^{\circ} \mathrm{C}$ results in a change in density of about $0.0003 \mathrm{~g} / \mathrm{mL}$ (gram per milliliter). A change in temperature from $10^{\circ}$ to $11^{\circ} \mathrm{C}$ results in a density change of about $0.0001 \mathrm{~g} / \mathrm{mL}$. Stable stratification is common in lakes and reservoirs where the density of the upper and lower strata of water differs by as 1 ittle as 0.0001 to $0.002 \mathrm{~g} / \mathrm{mL}$. Thus, temperature differences of $3^{\circ}$ to $4^{\circ} \mathrm{C}$ during the summer may result in stable stratification.

Thermal stratification assumes many patterns, depending upon the geographical location, climatological conditions, depth, surface area, and configuration of the lake or reservoir. During the winter, many deep lakes or reservoirs in the temperate zone are characteristically isothermal--that is, the water has a uniform temperature and density, and circulates freely. With the onset of spring, solar heating warms the incoming water and the water at the lake or reservoir surface causing a decrease in density. This warm surface water floats on the colder and denser water. As the surface water becomes progressively warmer, the density gradient increases and the depth to which wind can mix the water is diminished. Thus, water in the lake or reservoir commonly is separated into three fairly distinct strata:

(1) the epilimnion--a warm freely circulating surface stratum,

(2) the metal imnion--a middle stratum characterized by a rapid decrease in temperature with increases in depth, and

(3) the hypolimnion--a cold, stagnant lower stratum. 
Thermal stratification in deep lakes or reservoirs usually persists until fall, when a decrease in atmospheric temperature cools both the surface water in the reservoir and the inflow from streams. When the temperatures and densities of the epilimnion and metalimnion approach those of the hypolimnion, the resistance to mixing is reduced and complete mixing or overturn of the water occurs.

Incoming water to a lake or water al ready stored will flow into a layer where the water is most similar to its density. The water density is governed by the temperature, dissolved solids, and suspended solids. The movement of water to achieve density equil ibrium is referred to as a density current. Density currents may move water as an overflow on top of the water in storage, as an underflow at the bottom of the lake, or as an interflow plume at an intermediate depth.

Many shallow lakes become stratified during periods of calm but may be completely mixed by moderate winds. Moreover, shallow lakes are more quickly heated or cooled by atmospheric influences than deep lakes. Because Somerville Lake is shallow and probably easily mixed by moderate winds, the classical three-1 ayered stratification pattern was not observed on any occasion during the 16 water-qual ity surveys made.

On May 27, 1977, March 13, 1978, June 19, 1979, and May 21, 1980, there was a temperature gradient in the lower depths of the lake caused by cold inflows. Discharges, both inflow and outflow, were much higher during those surveys than at other times. The maximum temperature difference, $7^{\circ} \mathrm{C}$, occurred on June 19, 1979, when the lake water surface was at a record el evation of 246.09 feet, with a depth of 37 feet at site $A_{C}$.

The lake can be completely mixed at any time of the year, and was completely mixed during 8 of the 16 surveys. Isothermal conditions have been observed during all four seasons of the year. Wind action is the predominant mixing force all year; wind-induced waves mix the water from top to bottom. Water-temperature data at sites $A_{C}$ and $F_{C}$ at the time of the surveys are shown in figure 6 . Monthly mean air-temperature data for Somerville Dam al so are shown in figure 6 . These data show that the air and water temperatures are very similar for the same time period, varying by 1 ess than $3^{\circ} \mathrm{C}$ most of the time.

\section{Dissolved 0xygen}

Fish and other aquatic organisms require oxygen to maintain the metabolic processes that produce energy for egg and larvae development and normal activities. Moreover, some of the chemical constituents dissolved in water are related to dissolved-oxygen concentrations; therefore, dissolved oxygen is one of the most important factors that influence the qual ity of water in a lake or reservoir.

Water entering a lake or reservoir contains organic material derived from both natural sources and from man's waste. Bacterial stabilization of this organic material requires oxygen. Decaying trees, brush, and other oxidizable 


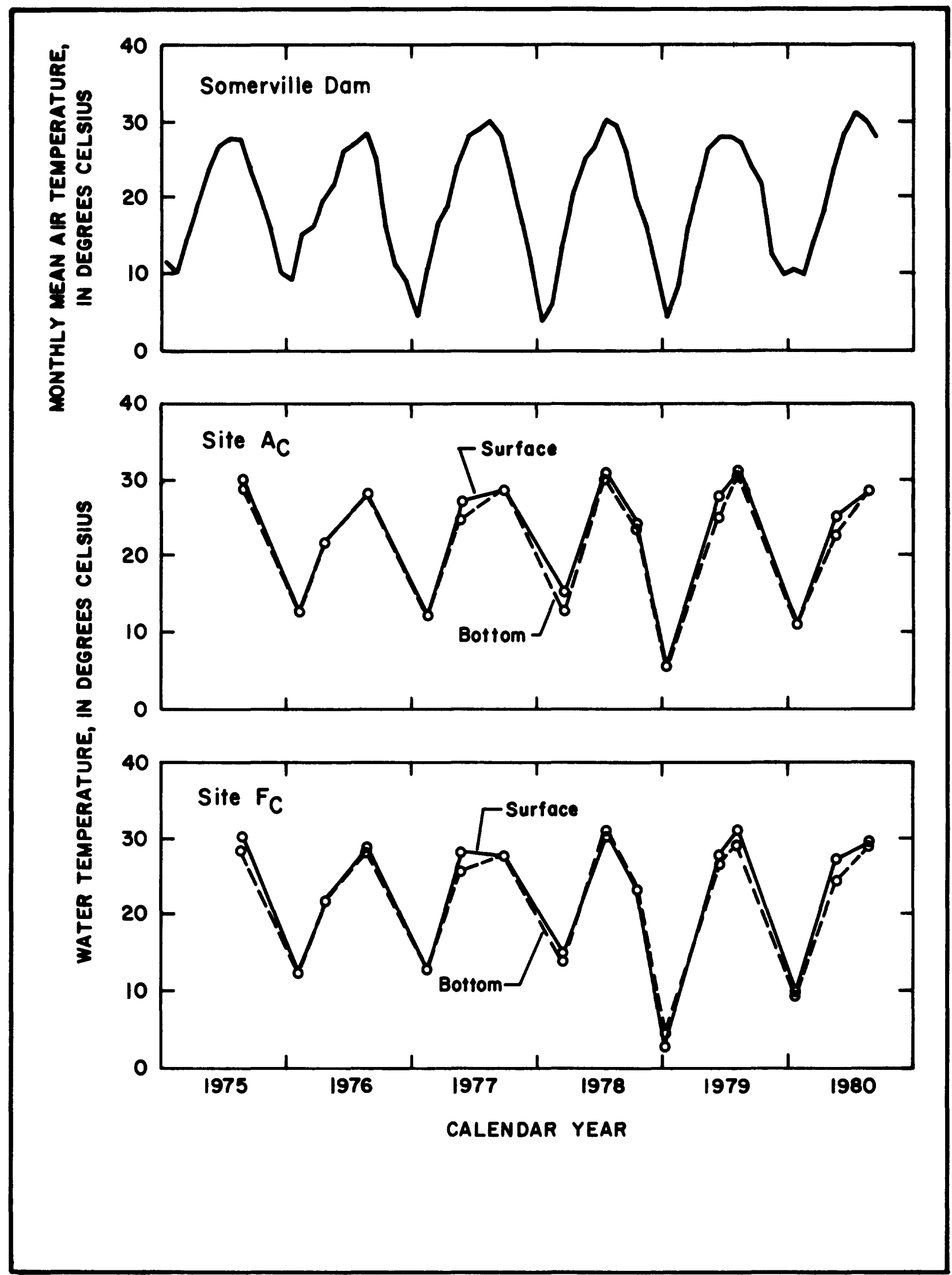

Figure 6. - Variations in monthiy mean air temperatures at Somervilie Dam and water temperatures at sites $A_{C}$ and $F_{C}$ during surveys 
material within the area inundated by a reservoir as well as decaying algae and other organic material produced within the reservoir exert an oxygen demand.

The distribution of dissolved oxygen in a lake or reservoir is related to thermal stratification. 0xygen enters the surface stratum by plant photosynthesis and by absorption from the atmosphere. During winter circulation, the water is continually circulated to the atmosphere, and dissolved oxygen used in the decomposition of organic matter is replenished. However, during spring and summer, thermal stratification results in a reduction of vertical circulation of the water. Oxygen used in the decomposition of organic material is not replaced in the hypolimnion, and a vertical dissolved-oxygen gradient develops.

Dissolved-oxygen data for Somerville Lake are given in figures 7 and 8 and in tables 2-17. The concentration of dissolved oxygen varied seasonally and areally as in most lakes in the temperate zone. The lake also follows a common pattern of rather high oxygen saturation during the winter. Even as spring approached during the survey of March 13,1978, no sample of water at any site, surface or bottom, showed less than 72-percent saturation with oxygen.

During the summer, especially in stratified lakes, it is common to observe high oxygen concentrations at the surface, a decline in the middle layer, and almost complete oxygen depletion at the bottom. All surveys in Somerville Lake showed a decline in oxygen concentrations from surface to bottom during the summer, and the rate of decline in the profiles varied considerably during each survey. However, most concentrations of dissolved oxygen at the bottom were in excess of 50-percent saturation during the summer surveys.

On August 20, 1976, at site $A_{C}$, the deepest sampling location on the lake, water near the surface had a dissolved oxygen concentration of $4.8 \mathrm{mg} / \mathrm{L}$ (62-percent saturation) while water near the bottom had a dissolved oxygen concentration of $4.4 \mathrm{mg} / \mathrm{L}$ (56-percent saturation). The temperature was $28^{\circ} \mathrm{C}$ from surface to bottom. This profile is similar to those obtained for the winter months.

The depth-averaged concentration of dissolved oxygen at site $A_{C}$, near the dam, was about $5.7 \mathrm{mg} / \mathrm{L}$ during the summer and about $10.6 \mathrm{mg} / \mathrm{L}$ during the winter. The depth-averaged concentrations of dissolved oxygen at site $F_{C}$, at the headwaters, were about $5.8 \mathrm{mg} / \mathrm{L}$ during the summer and $10.0 \mathrm{mg} / \mathrm{L}$ during the winter.

The varied, yet high dissolved oxygen concentrations show Somerville Lake to be a dynamic impoundment. During the winter the cooling of the upper layer creates density currents that result in circulation and aeration of the entire body of water. Wind action on the shallow lake, density currents caused by the daily heating and cooling of surface water, and the circulation of inflow and outflow water promote mixing in the lake during the entire year. The yearly volume of water flowing through the lake averages one and one-half times the volume of the lake. 


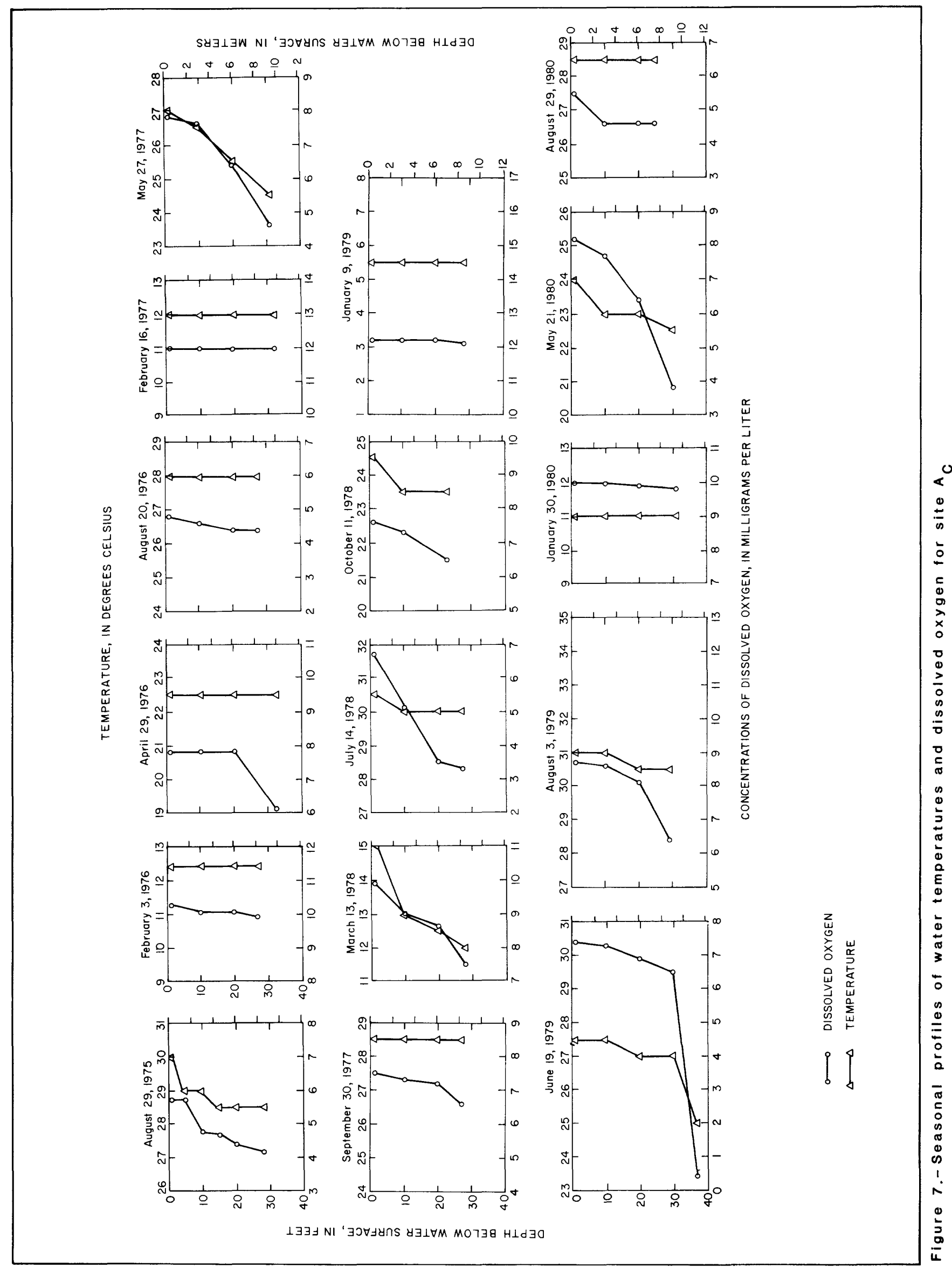




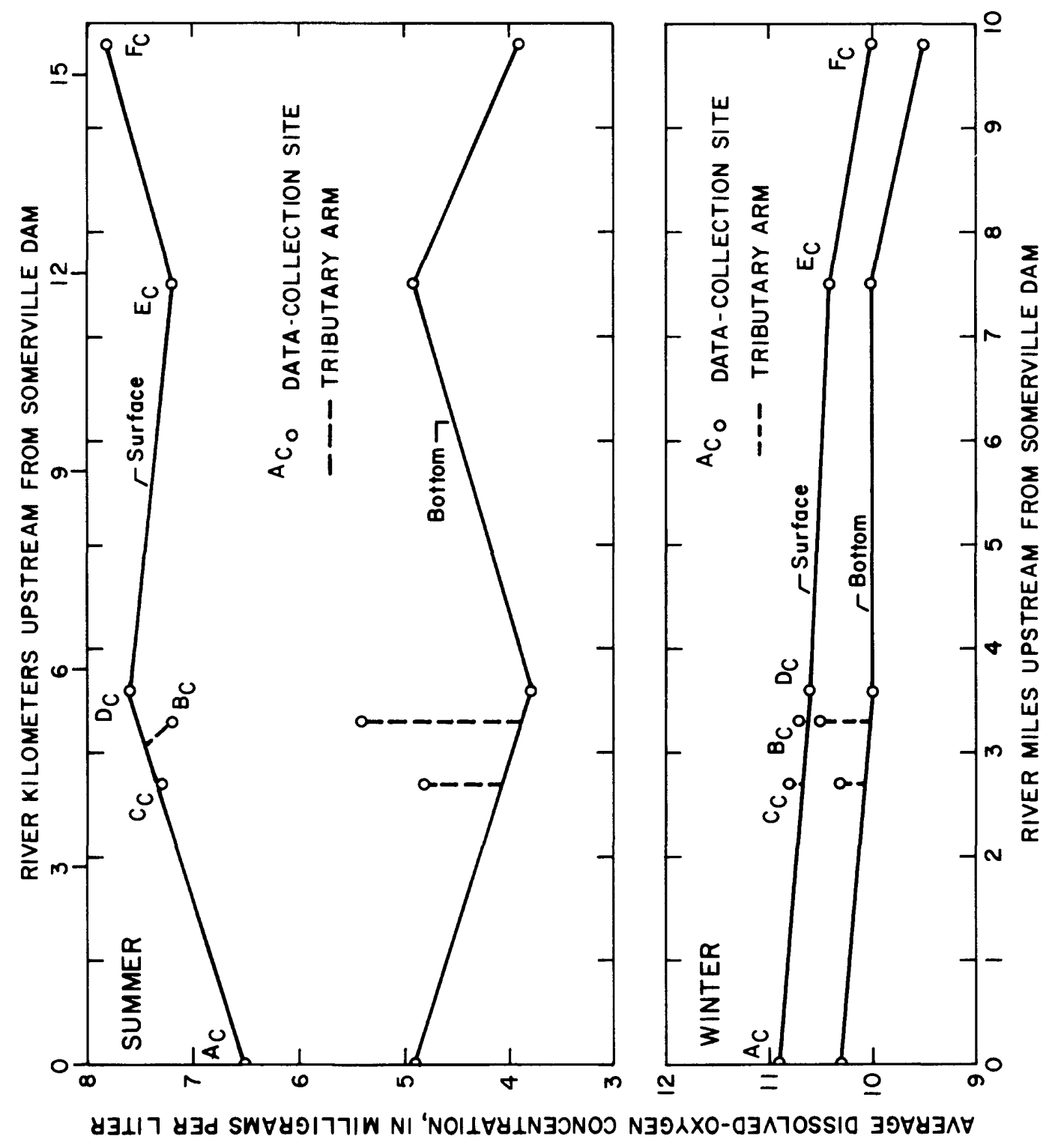


The occurrence and distribution of dissolved iron and dissolved manganese in lake waters are inversely related to the dissolved oxygen concentrations. During thermal stratification, the oxygen utilized in the decomposition of organic material is not replenished, thus in the period of anaerobic decomposition that follows, reducing conditions often result in the dissolution of 1 arge amounts of iron and manganese. The concentrations of iron and manganese in the bottom waters of Lake Somerville increased during periods of reduced circulation but decreased as soon as circulation increased. In any season throughout the year, water near the surface of the lake and water near the bottom during periods of circulation at all sites, except the headwaters at site $F_{C}$, usually contain less than $50 \mu \mathrm{g} / \mathrm{L}$ (micrograms per 1 iter) of dissolved iron and $40 \mu \mathrm{g} / \mathrm{L}$ of dissol ved manganese (figs. 9-11). However, during short periods of stagnation, the concentrations of both constituents near the bottom of the lake increased in the upstream direction in response to decreases in the concentration of dissolved oxygen. For example, on rare occasions at site $F_{C}$ when the dissolved oxygen concentration decreased significantly, as on August 29, $1975(1.7 \mathrm{mg} / \mathrm{L})$ and July 14, $1978(2.8 \mathrm{mg} / \mathrm{L})$, the dissolved iron concentrations were $790 \mu \mathrm{g} / \mathrm{L}$ and $1,100 \mu \mathrm{g} / \mathrm{L}$, and the dissolved manganese concentrations were $820 \mu \mathrm{g} / \mathrm{L}$ and $520 \mu \mathrm{g} / \mathrm{L}$. The concentration of oxygen decreases in the upstream direction probably when small amounts of organic material, brought by inflow water, are oxidized. The amounts are small enough that the oxygen demand is satisfied soon after entry into the lake.

The dissolved iron concentrations near the bottom at site $F_{C}$, a shallow site in the headwaters of the lake, ranged from 0 to $1,100 \mu \mathrm{g} / \mathrm{L}$ and averaged about $250 \mu \mathrm{g} / \mathrm{L}$. Di ssol ved manganese concentrations near the bottom at this site ranged from 0 to $820 \mu \mathrm{g} / \mathrm{L}$ and averaged about $180 \mu \mathrm{g} / \mathrm{L}$. These are the highest averages of all sites observed on this lake. The organic material in the slow moving inflow probably used most of the oxygen; under these reducing conditions, the iron and manganese at the bottom become soluble.

At site $A_{C}$ near Somerville Dam, the concentrations of dissolved iron in water near the bottom ranged from 0 to $230 \mu \mathrm{g} / \mathrm{L}$ and averaged about $50 \mu \mathrm{g} / \mathrm{L}$. The concentrations of dissolved manganese ranged from 0 to $440 \mu \mathrm{g} / \mathrm{L}$ and averaged about $90 \mu \mathrm{g} / \mathrm{L}$. There was no buildup of concentrations of dissolved iron or dissolved manganese at site $A_{C}$ during the period of record (fig. 12).

\section{Total Inorganic Nitrogen and Total Phosphorus}

The average summer concentrations of total inorganic nitrogen $\left(\mathrm{NO}_{2}+\mathrm{NO}_{3}+\mathrm{NH}_{4}\right.$ as $N$ ) varied little throughout the reservoir (fig. 13). At site $A_{C}$, the average surface concentration was $0.01 \mathrm{mg} / \mathrm{L}$ and the average bottom concentration was $0.02 \mathrm{mg} / \mathrm{L}$. At site $D_{C}$ in the central part of the lake, the average concentrations increased slightly to $0.03 \mathrm{mg} / \mathrm{L}$ at the surface and $0.04 \mathrm{mg} / \mathrm{L}$ at the bottom. At site $F_{C}$, the average concentrations were about the same as for the site near the dam, $0.01 \mathrm{mg} / \mathrm{L}$ at the surface and $0.02 \mathrm{mg} / \mathrm{L}$ at the bottom.

There al so was little difference in the average concentration of total inorganic nitrogen between the winter surface and bottom samples; however, 


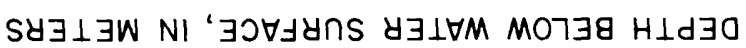

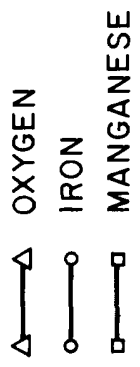
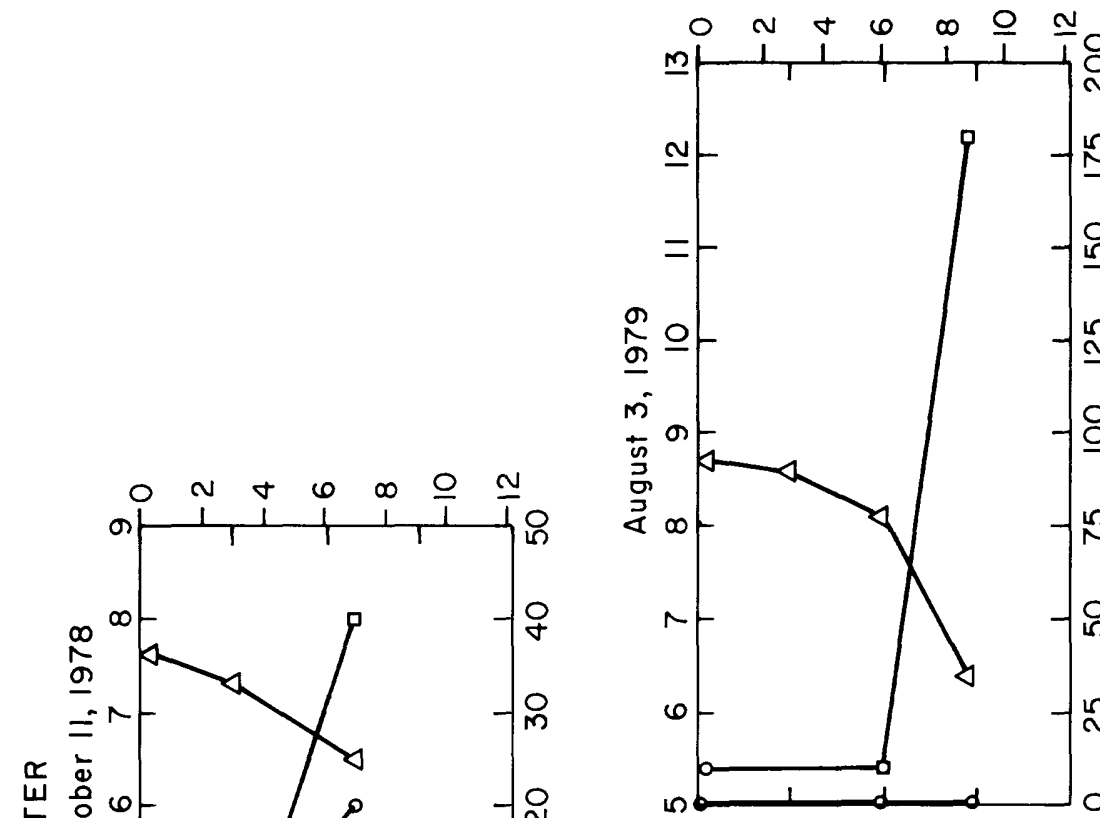

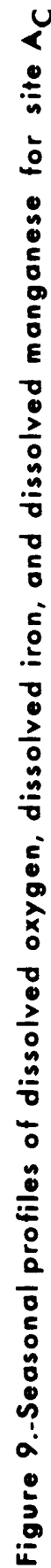




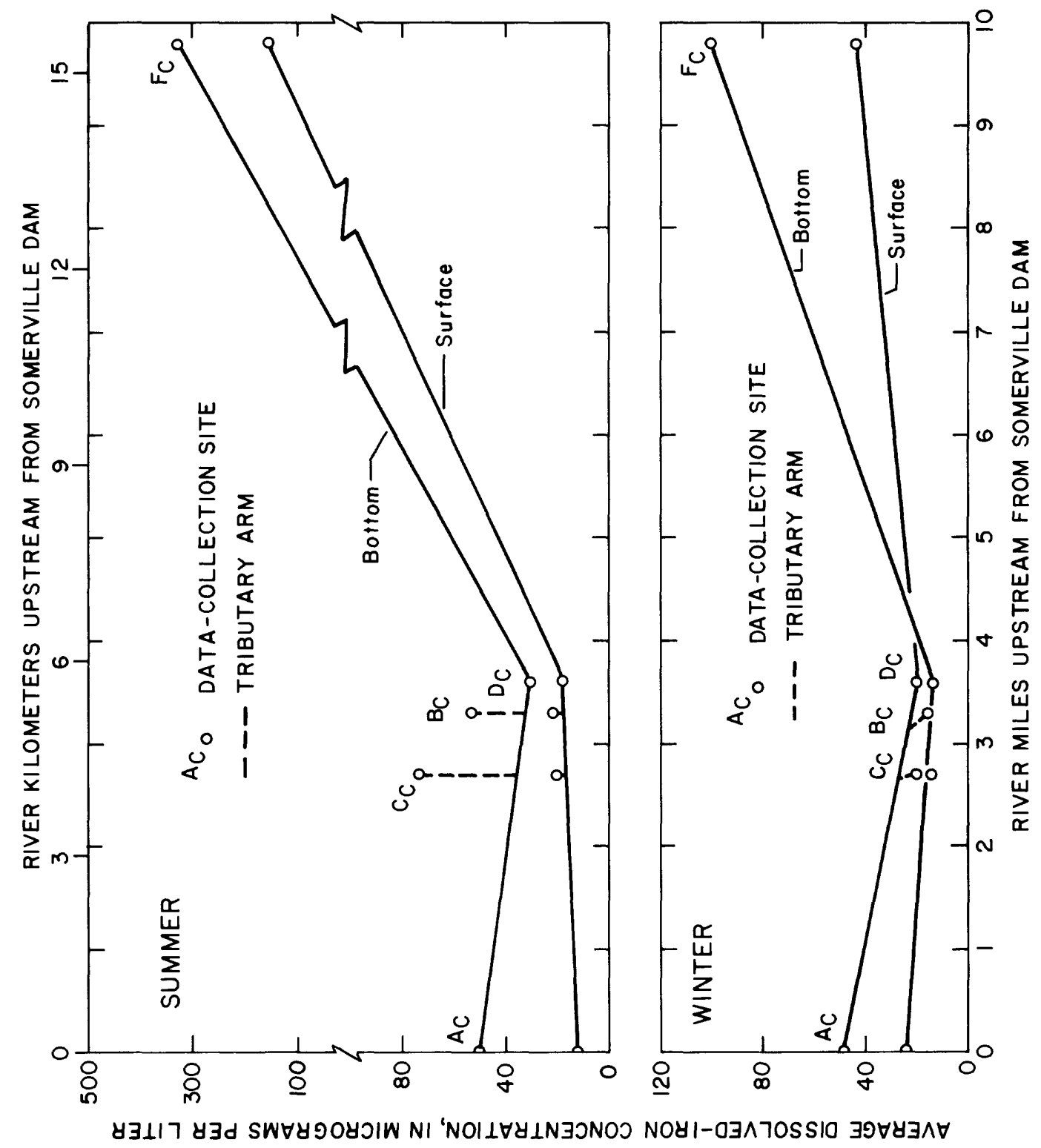

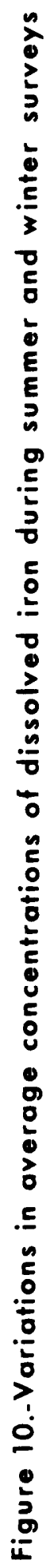




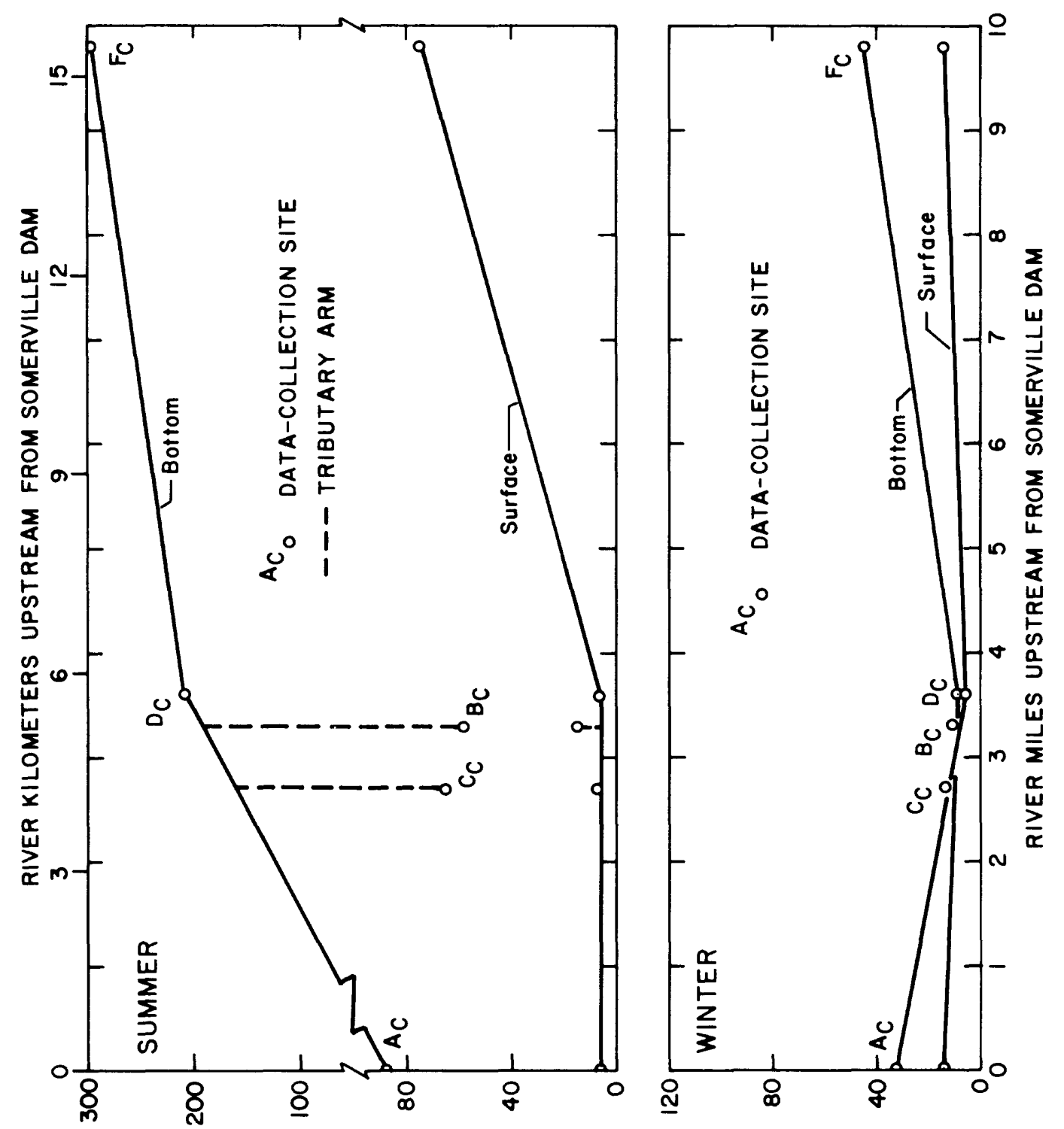

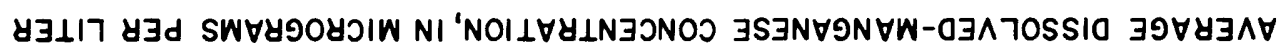



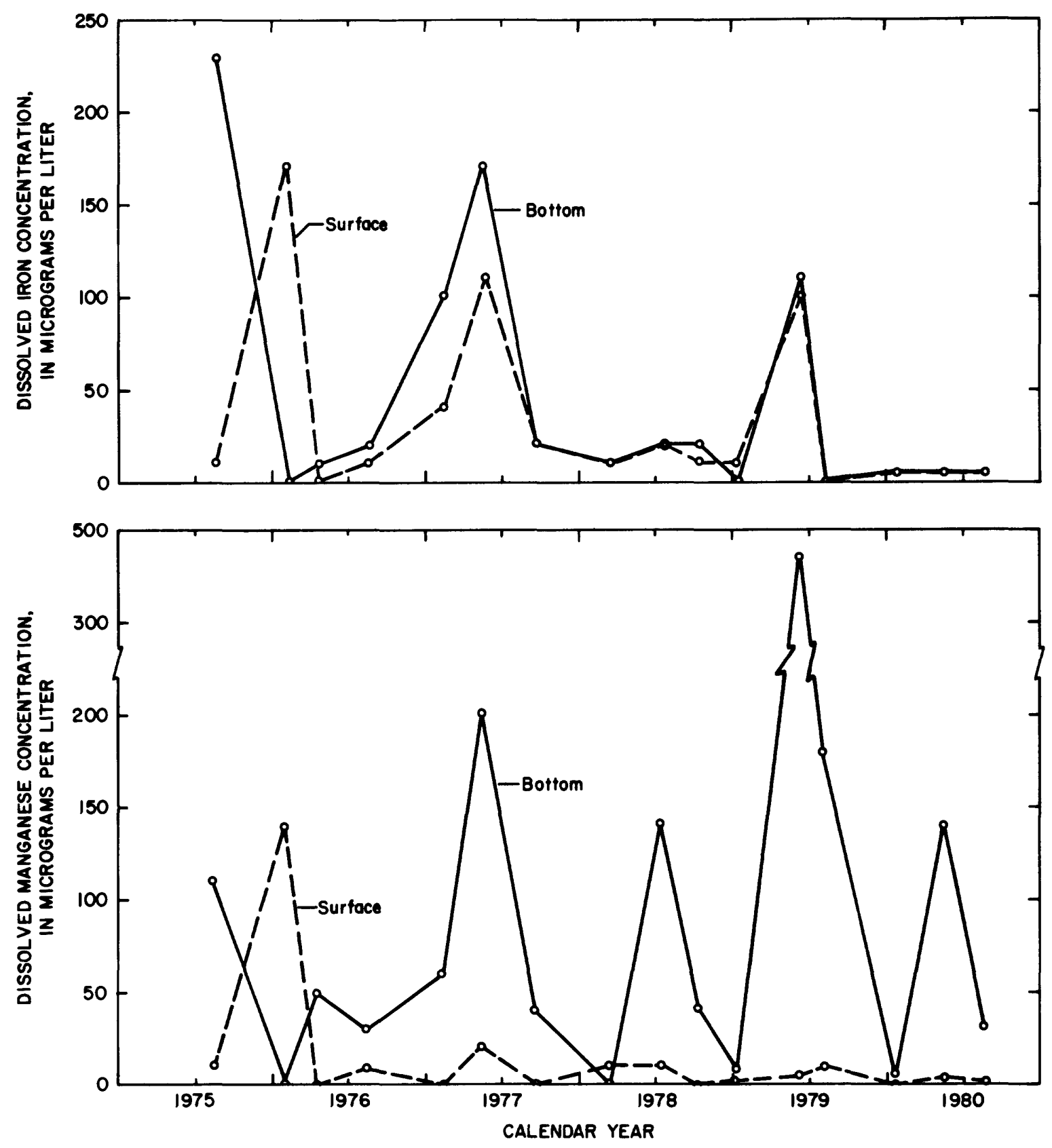

Figure 12.-Variations in concentrations of dissolved iron and dissolved manganese at site $A_{C}$, August 1975 -August 1980 


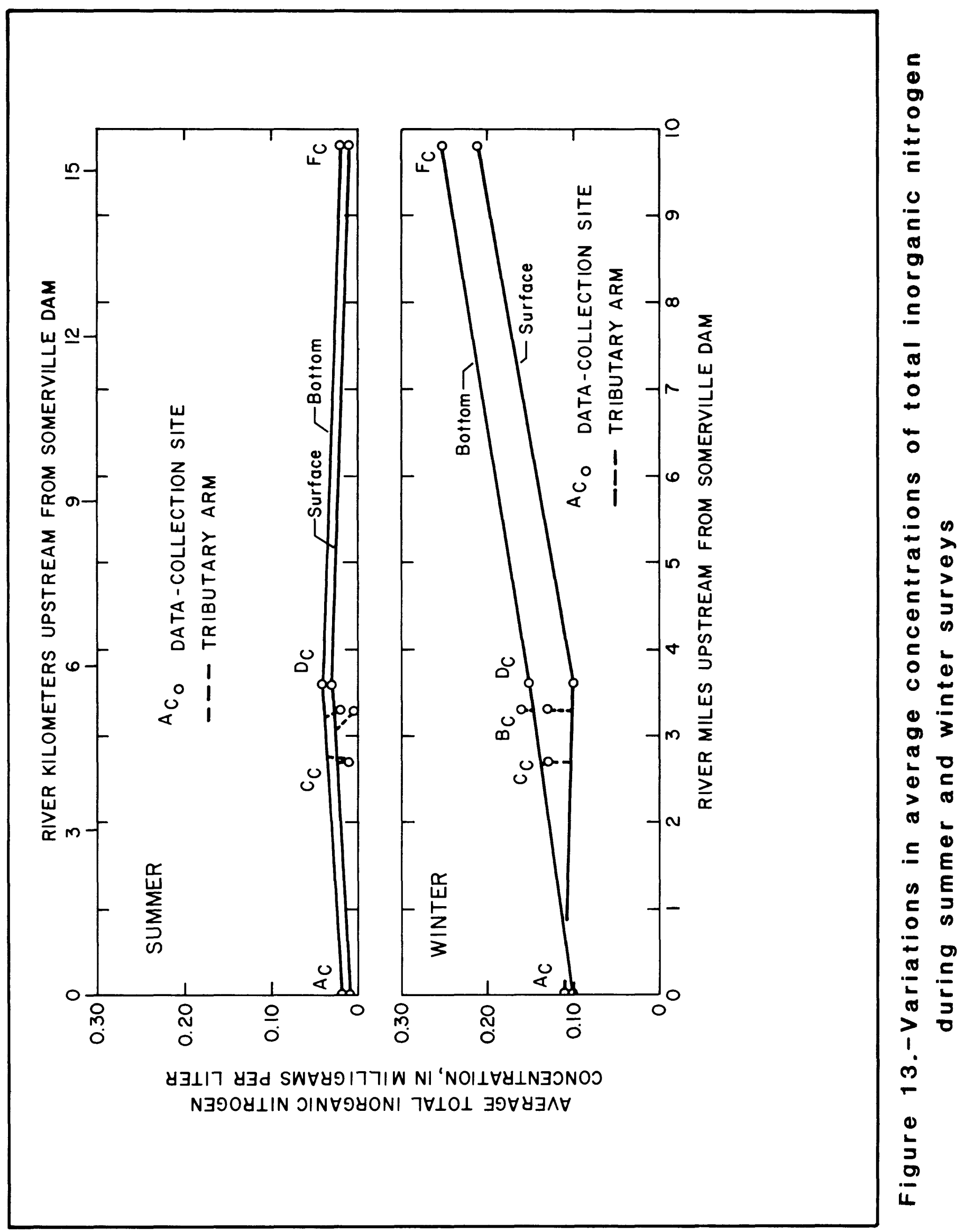


winter concentrations generally were larger than summer concentrations. During the winter, the average concentration of total inorganic nitrogen was 0.11 $\mathrm{mg} / \mathrm{L}$ for surface samples and $0.10 \mathrm{mg} / \mathrm{L}$ for bottom samples at site $A_{C}$. The trends were reversed at site $D_{C}$ with concentrations of $0.10 \mathrm{mg} / \mathrm{L}$ at the surface and $0.15 \mathrm{mg} / \mathrm{L}$ at the bottom. At site $F_{C}$, the concentrations were somewhat higher and averaged $0.21 \mathrm{mg} / \mathrm{L}$ for the surface samples and $0.25 \mathrm{mg} / \mathrm{L}$ for the bottom samples.

There was 1 ittle seasonal variation in the average concentration of total phosphorus from Somerville Lake waters (fig. 14). Surface and bottom total phosphorus concentrations during summer and winter averaged about the same, 0.04 and $0.06 \mathrm{mg} / \mathrm{L}$, near the dam, in the central body of the lake, and in the lower tributary arms. During the summer, the surface total phosphorus concentrations in this downstream area averaged $0.04 \mathrm{mg} / \mathrm{L}$ and the bottom average was 0.05 $\mathrm{mg} / \mathrm{L}$. At site $F_{C}$, the surface concentrations averaged $0.09 \mathrm{mg} / \mathrm{L}$ and the bottom concentrations averaged $0.11 \mathrm{mg} / \mathrm{L}$ during the summer, while during the winter, the surface concentrations averaged $0.12 \mathrm{mg} / \mathrm{L}$ and the bottom concentrations averaged $0.13 \mathrm{mg} / \mathrm{L}$. These concentrations are about double the concentrations that were found at site $A_{C}$.

Homogeneous or nearly homogeneous total inorganic nitrogen and total phosphorus concentrations (usually less than $0.03 \mathrm{mg} / \mathrm{L}$ ) can occur at any time of the year, from surface to bottom, either at the deepest water near the dam, site $A_{C}$, or at the shallow inflow site $F_{C}$. For example, on January 9, 1979, at site $A_{C}$, total inorganic nitrogen was $0.11 \mathrm{mg} / \mathrm{L}$ at surface and bottom, and total phosphorus was $0.04 \mathrm{mg} / \mathrm{L}$ at surface and bottom. At the same site on August 3, 1979, the concentration of total inorganic nitrogen was 0.00 $\mathrm{mg} / \mathrm{L}$ at surface and $0.01 \mathrm{mg} / \mathrm{L}$ at the bottom, while the concentration of total phosphorus was $0.04 \mathrm{mg} / \mathrm{L}$ at surface and bottom (figs. 15-18).

At site Ac from August 1975 to March 1978 there was an apparent trend of slightly increasing total phosphorus concentrations to about $0.10 \mathrm{mg} / \mathrm{L}$. There was a slight decrease from March 1978 to August 1980 to about $0.05 \mathrm{mg} / \mathrm{L}$. The apparent trend at site $F_{C}$ was a gradual increase of total phosphorus concentration to about $0.17 \mathrm{mg} / \mathrm{L}$ during the whole study except for one temporary decrease as inflow increased during the record elevation in the lake in June and July 1979.

Concentrations of total inorganic nitrogen fluctuated from zero to near zero in late summer or early fall to peaks of $0.1,0.2$ and $0.3 \mathrm{mg} / \mathrm{L}$ in the spring, while concentrations of total phosphorus showed 1 ittle change from one season to the next, usually less than $0.03 \mathrm{mg} / \mathrm{L}$. Though some of the changes in concentration of the nutrients can be attributed to plant assimilation and release, other changes may be attributed to their relative sol ubilities in well oxygenated water. Phosphorus compounds are less soluble, tend to absorb on the sediment, and remain available to go back into solution when the dissol ved-oxygen concentration is low. Their low solubilities in oxygen-rich water tend to keep concentrations at relatively low levels. On the other hand, nitrogen compounds are more abundant and more sol uble in oxygenated water. During periods of large releases from the lake, most of the total inorganic nitrogen, being in solution, is flushed out through the gates, while phosphorus is trapped in the bottom sediments of the lake. 


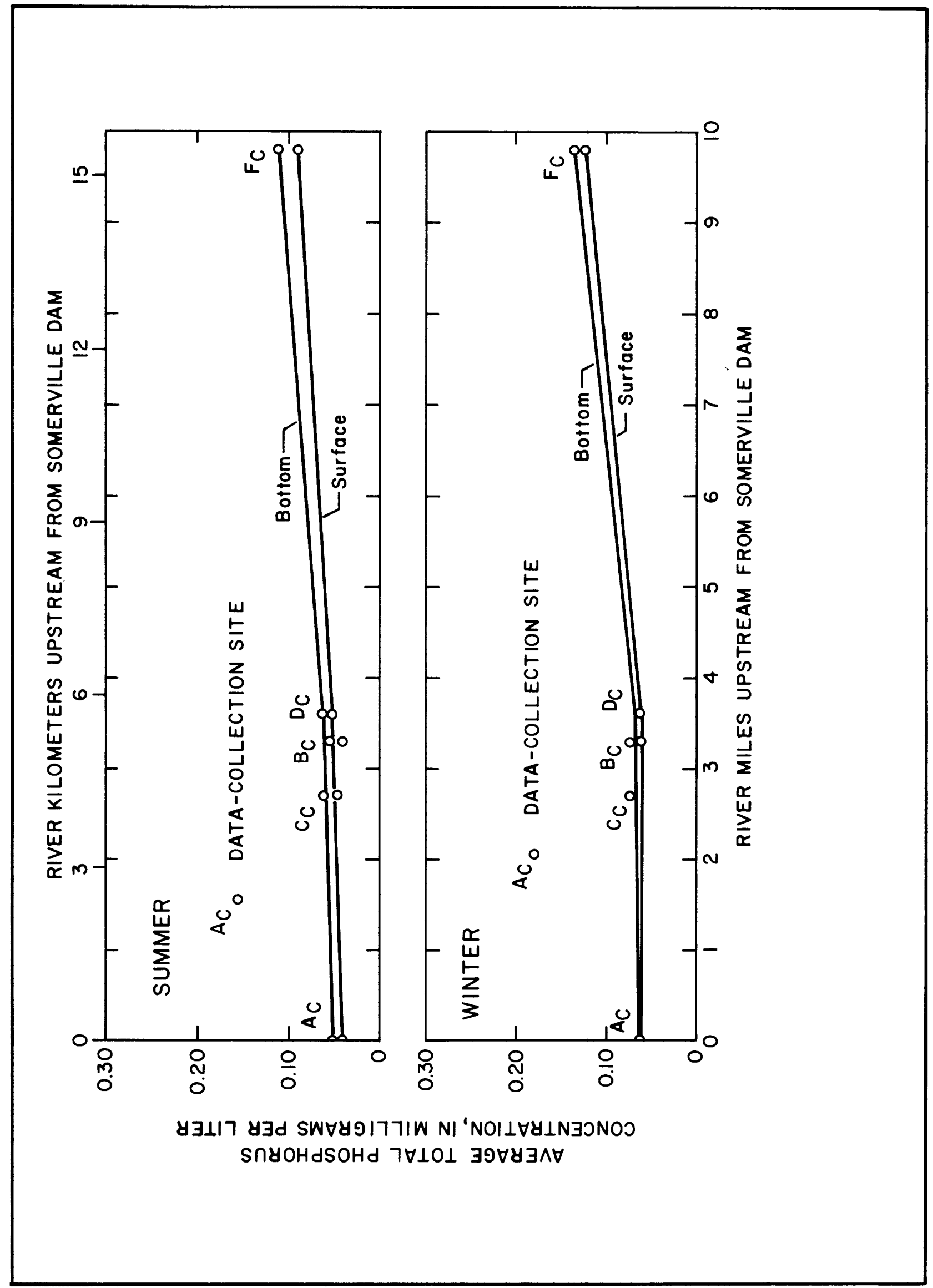

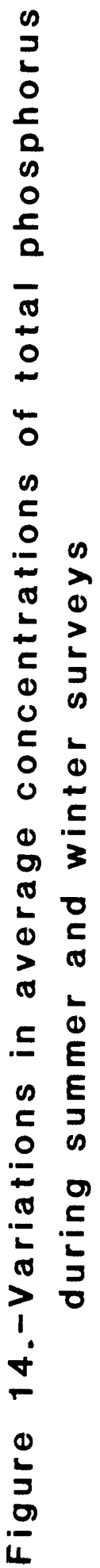


TEMPERATURE, IN DEGREES CELSIUS

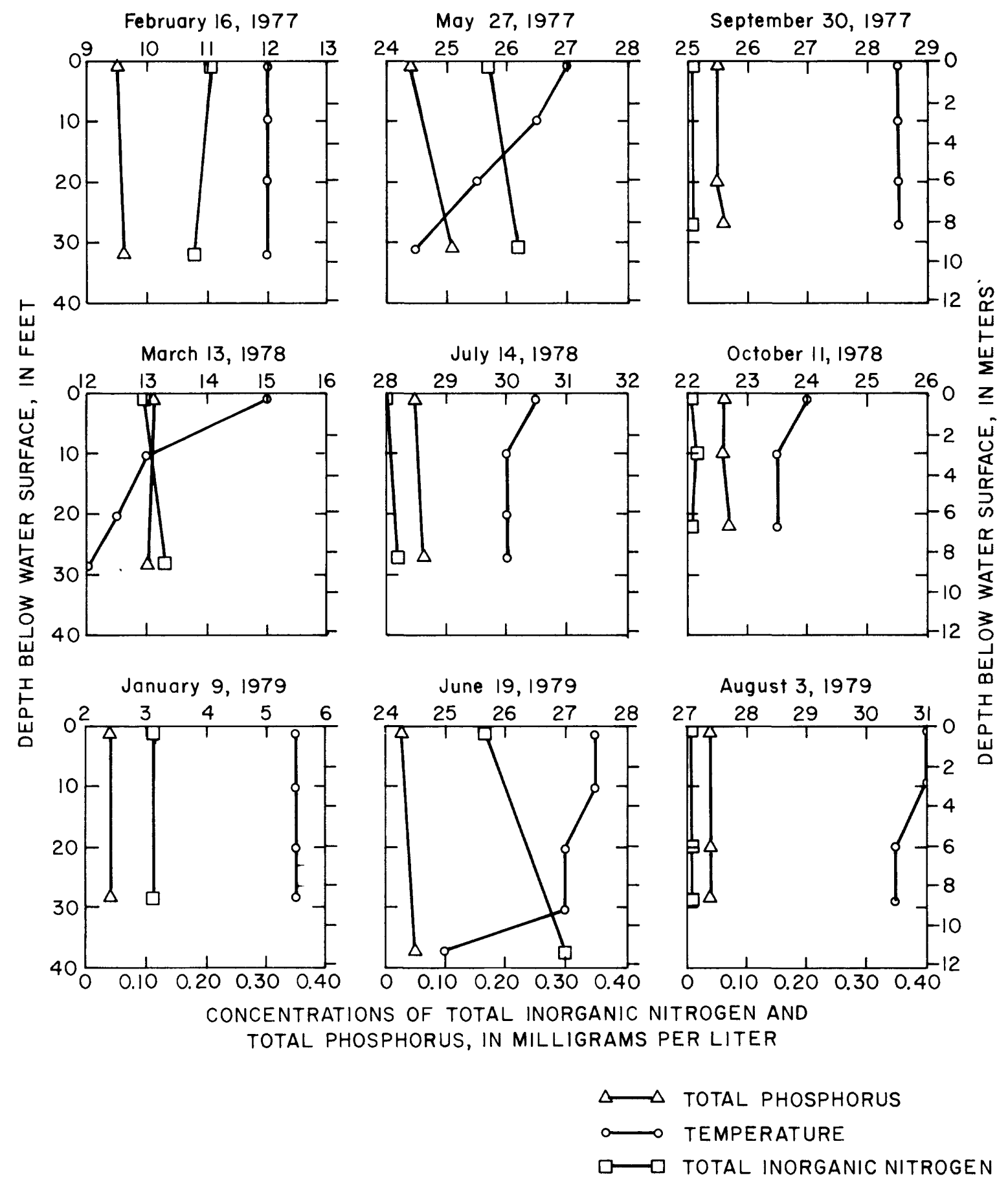

Figure 15.-Seasonal profiles of water temperatures, total inorganic nitrogen, and total phosphorus for site $A_{C}$ 
TEMPERATURE, IN DEGREES CELSIUS
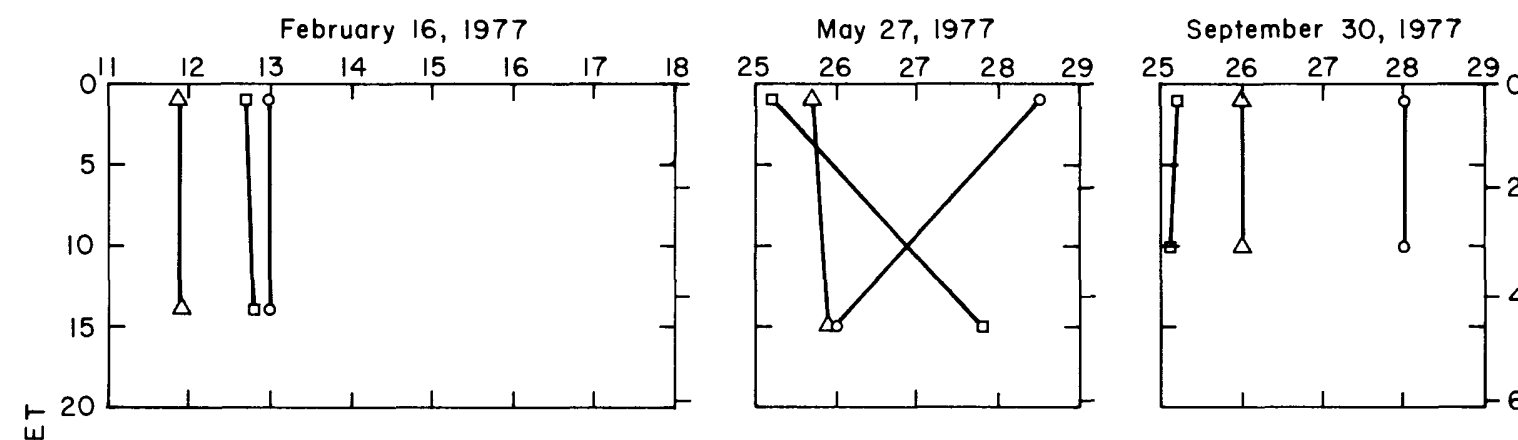

$$
\begin{aligned}
& u \\
& w \\
& \underline{u} \\
& \underline{z}
\end{aligned}
$$
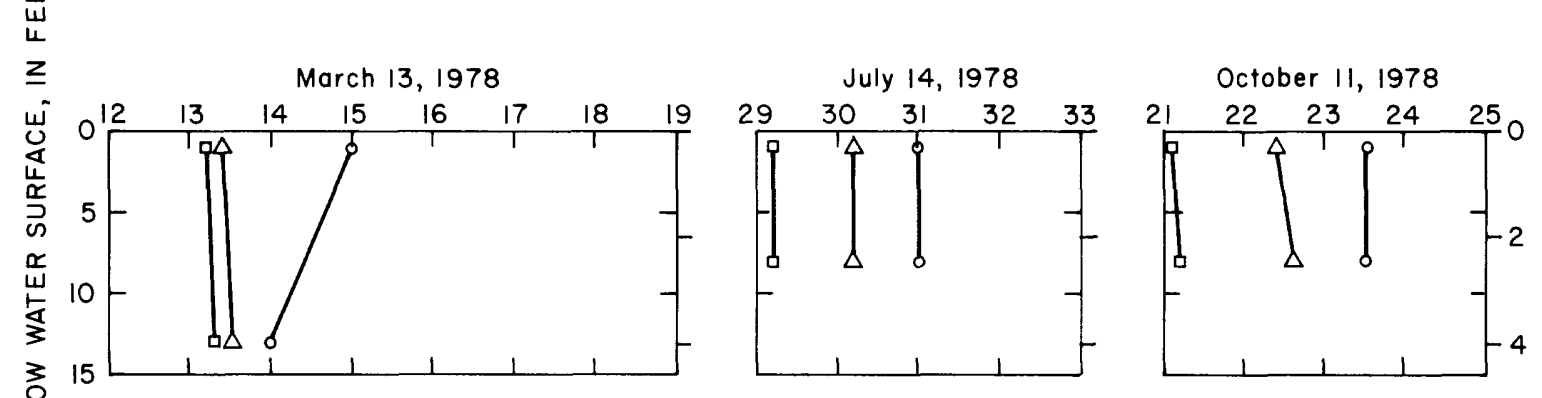

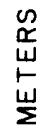

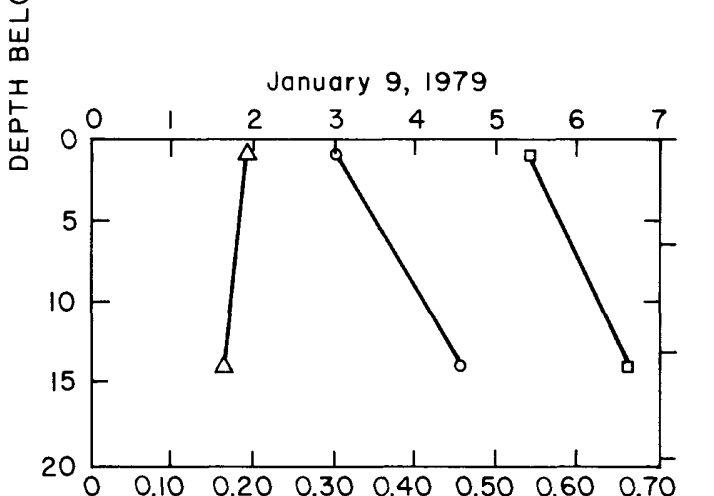

CONCENTRATIONS OF
TOTAL PHOSPHORUS,
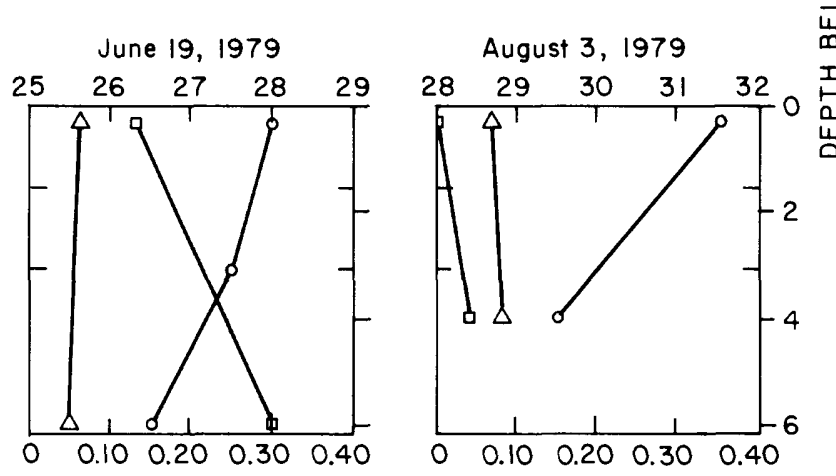

$\triangle$ TOTAL PHOSPHORUS

$\leadsto$ TEMPERATURE

$\square$ TOTAL INORGANIC NITROGEN

Figure 16.-Seasonal profiles of water temperatures, total inorganic nitrogen, and total phosphorus for site $F_{C}$ 

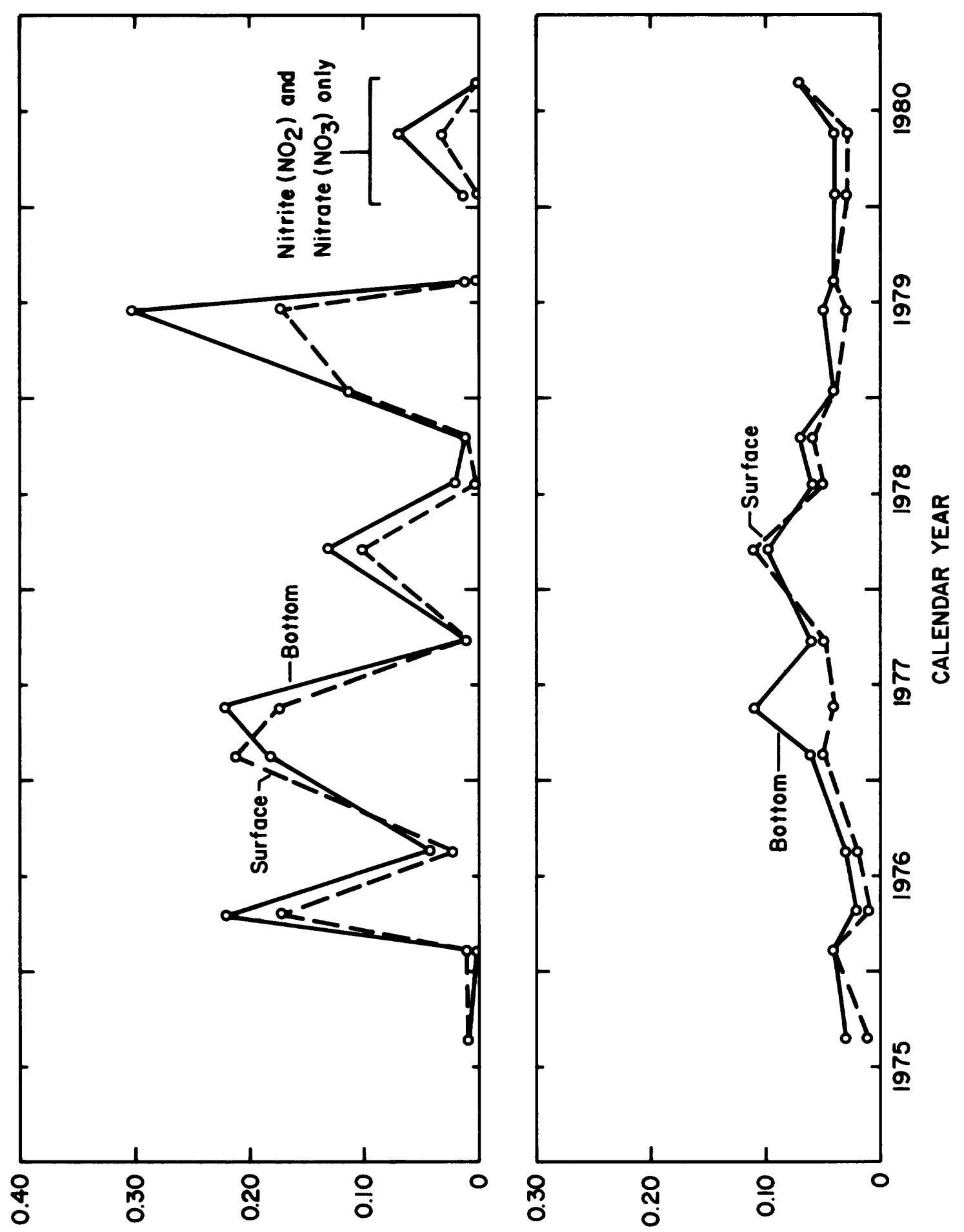

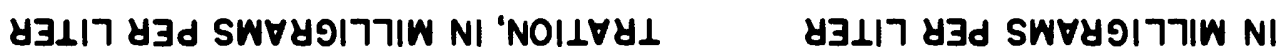

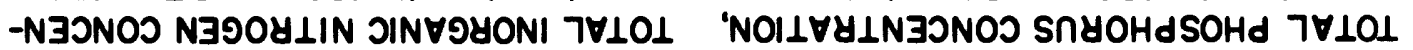




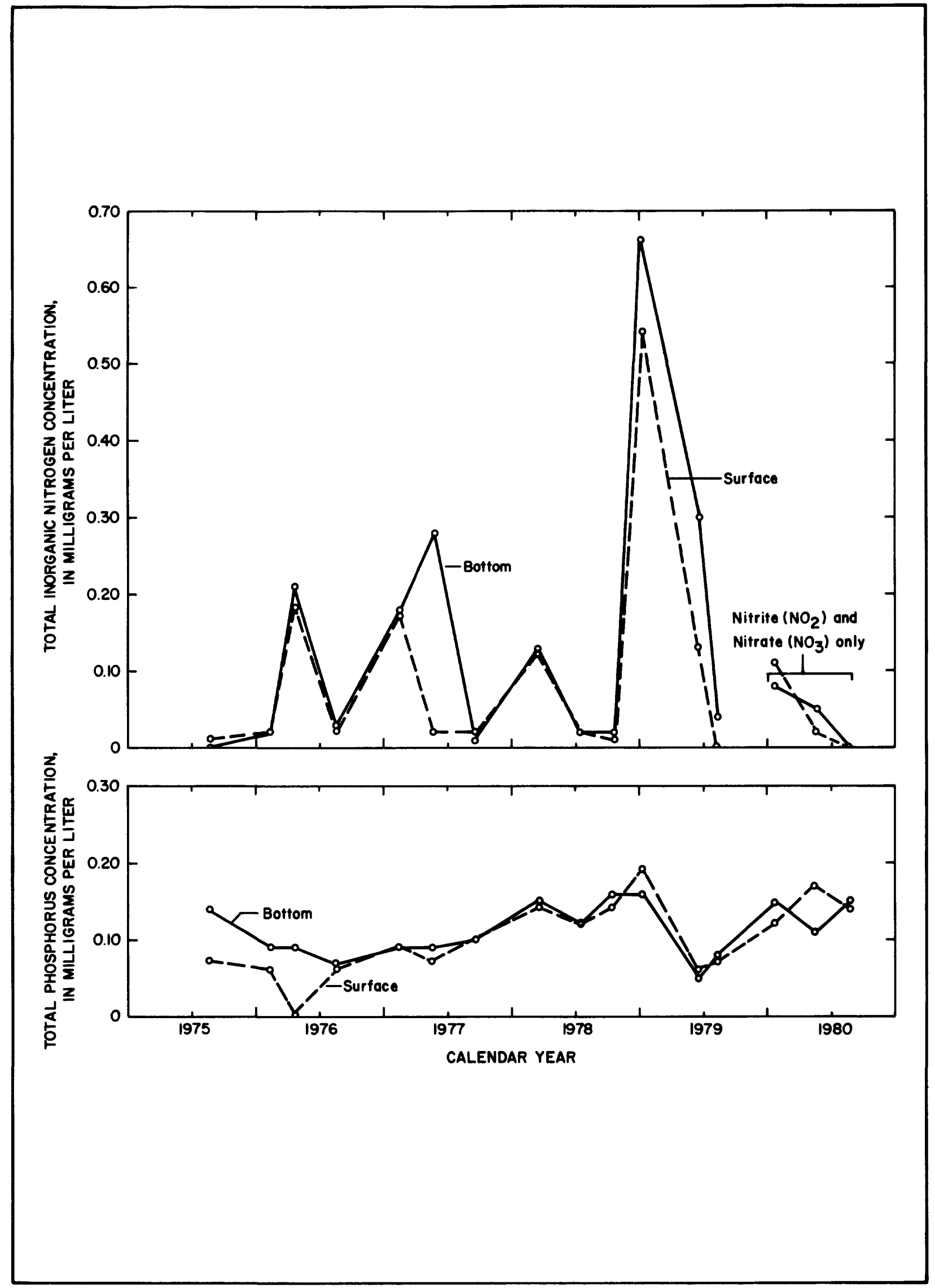

Figure 18.-Variations in concentrations of total inorganic nitrogen and total phosphorus at site FC, August 1975-August 1980 


\section{Dissolved Solids, Dissolved Chl oride,}

Dissol ved Sulfate, and Hardness

Some of the more important properties or constituents that affect the utility of a lake or reservoir as a public water supply include dissolved solids, dissolved chloride, dissolved sulfate, and hardness. Because dissolved constituents are in the ionic state, they conduct electricity. Therefore, a correlation can be made between the dissolved solids and the specific conductance of the water (Hem, 1970, p. 96-103). Over short time periods and in the absence of outside influences, the relative concentrations of the major constituents maintain a nearly constant ratio to specific conductance. Therefore specific conductance can be used to detect and document variations of selected constituents in the water of a lake. During each lake survey, the specific conductance of water at each data-collection site was determined at depth intervals of 5 to 10 feet. From these data and from results of analyses for dissolved solids, dissolved chloride, dissolved sulfate, and hardness for samples collected near the surface and bottom at selected sites (tables 2-17), 1 inear-regression equations were developed to estimate the volume-weighted average concentration of dissolved constituents. Data in figure 19 show that vol ume-weighted average concentrations of the total hardness in Somerville Lake ranged from 75 to $140 \mathrm{mg} / \mathrm{L}$ and averaged $110 \mathrm{mg} / \mathrm{L}$ expressed as calcium carbonate, placing the water in the moderately hard to hard (61 to $180 \mathrm{mg} / \mathrm{L}$ ) classification (Hem, 1970, p. 225). The vol ume-weighted average concentrations of dissolved solids ranged from 139 to $292 \mathrm{mg} / \mathrm{L}$ and averaged about 220 $\mathrm{mg} / \mathrm{L}$. Vol ume-weighted average concentrations of dissolved chloride ranged from 20 to $68 \mathrm{mg} / \mathrm{L}$ and averaged $43 \mathrm{mg} / \mathrm{L}$. Volume-weighted average concentrations of dissolved sulfate ranged from 30 to $130 \mathrm{mg} / \mathrm{L}$ and averaged $63 \mathrm{mg} / \mathrm{L}$.

The volume-weighted average concentrations of these constituents changed little throughout the period of record. The lowest concentrations were found on June 19, 1979, after a period of large inflow, when the el evation reached a record of 246.09 feet, and the lake held 270,000 acre-feet of water.

The variations in concentrations of dissolved solids differed only slightly during summer and winter surveys (figure 20). Data show that the average concentrations of dissolved solids in water at the surface differ by less than 10 $\mathrm{mg} / \mathrm{L}$ from water at the bottom. There was an increase of only about $20 \mathrm{mg} / \mathrm{L}$ in the average dissolved solids concentrations from site $A_{C}$ (at the dam) to site $\mathrm{F}_{C}$ (the headwater) during the summer and only about a $30-\mathrm{mg} / \mathrm{L}$ increase during the winter. Data show that significant stratification of the principle dissolved constituents within the lake does not occur, and the lake water is well mixed most of the time. The concentrations of the principal constituents are low enough that Somerville Lake is considered an excellent source of water for municipal, industrial, and agricultural use. In fact, they are lower than public drinking water standards for Texas: Chloride - $300 \mathrm{mg} / \mathrm{L}$, sulfate $300 \mathrm{mg} / \mathrm{L}$, and total dissolved solids - 1,000 mg/L (Texas Department of Heal th Drinking Water Standards, revised November 30, 1977).

\section{Water Transparency}

Aquatic plants require light for photosynthesis. The principal factors that affect the depth of light penetration in a lake include color and turbid- 


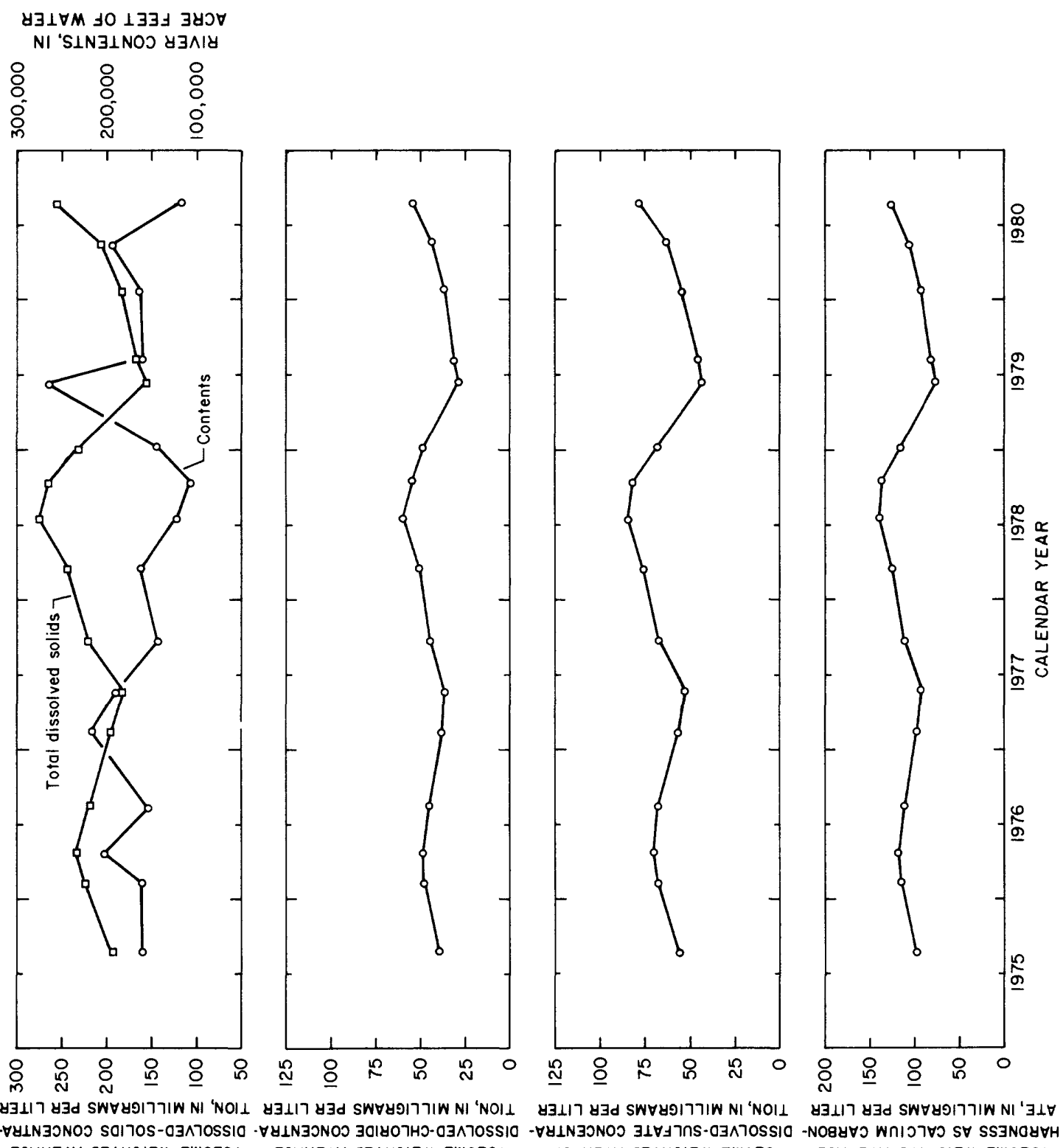

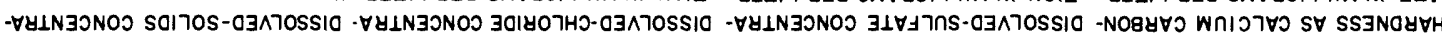

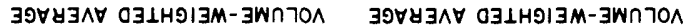



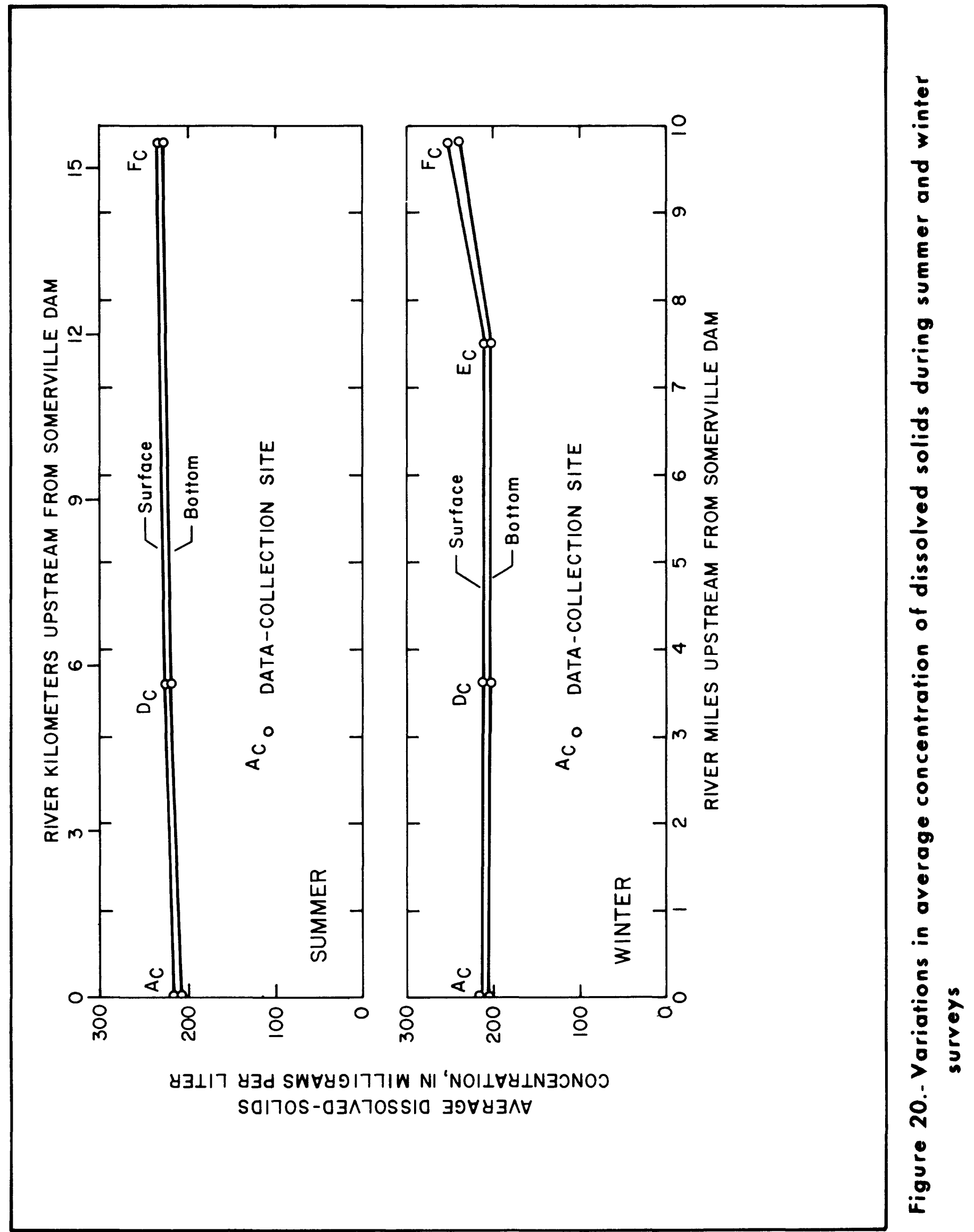
ity of the water. Turbidity is dependent upon both the concentration and size of suspended particles. The suspended materials may consist of suspended sediment from inflowing streams and living or dead microscopic plants and animals or their detritus.

Water transparency was measured in Somerville Lake by lowering a Secchi disc into the water and averaging the greatest depth at which it could be visually detected on lowering and raising it. The Secchi disc readings on the more turbid section of the headwaters, at site $F_{C}$, averaged $0.30 \mathrm{~m}$, increased to $0.63 \mathrm{~m}$ at site $D_{C}$, and were greatest near the dam at site $A_{C}$, where the average was $0.75 \mathrm{~m}$. An average reading of $0.59 \mathrm{~m}$ for the tributary site $C_{C}$ was comparable to the central site.

The increase in transparency toward the dam indicates that some suspended material is desposited in the upstream part of the lake. Some of the material desposited may again be placed in suspension either by wave action on the shallow lake or by currents during large inflows or discharges.

Phytopl ankton

Phytoplankton data were collected at sites $A_{C}$ and $F_{C}$ on two surveys during 1980. No interpretation can be made until much more phytoplankton data are collected.

\section{SUMMARY}

Somerville Lake is a shallow lake, with a mean depth of 14 feet. The depth of the submerged channel of Yegua Creek usually is less than 35 feet, and in most areas of the lake outside the submerged channel, the depth is less than 10 feet.

During winter the water near the surface cools, increases in density, and replaces the warmer and lighter water below the surface. Due to the rather shallow depth, this circulation pattern keeps the lake almost isothermal and well mixed during the winter. Throughout the year, wind action and density currents caused by daily heating and cooling of the surface water, and the large vol ume of inflow and discharge ratio to lake vol ume all contribute to keep Somerville Lake well mixed. Inlike deep lakes, the typical pattern of thermal stratification exists only for short periods. During the 161 ake surveys made, the classical three-l ayered stratification pattern of deeper lakes was not observed.

Like most lakes in the temperate zone, the concentration of dissolved oxygen varied seasonally and areally. The lake al so followed the common pattern of high oxygen saturation during the winter. During the summer the dissolved oxygen concentrations continued to be unseasonably high at greater depths of the lake. Most dissolved oxygen concentrations near the bottom were in excess of 50-percent saturation.

The depth-averaged concentration of dissolved oxygen at the deepest site $A_{C}$ near the dam, averaged $5.7 \mathrm{mg} / \mathrm{L}$ during the summer and about $10.6 \mathrm{mg} / \mathrm{L}$ during 
the winter. The dissolved oxygen concentrations at the headwaters site $\mathrm{F}_{\mathrm{C}}$ averaged about $5.8 \mathrm{mg} / \mathrm{L}$ during the summer and $10.0 \mathrm{mg} / \mathrm{L}$ during the winter.

The occurrence and distribution of dissolved iron and dissolved manganese in Somerville Lake are inversely related to the dissolved-oxygen concentrations of the water. With the year-round high dissolved oxygen saturation near the surface and near the bottom, dissolved iron concentrations were less than 50 $\mu \mathrm{g} / \mathrm{L}$ and dissolved manganese concentrations were less than $40 \mu \mathrm{g} / \mathrm{L}$. However during short periods of stagnation, the concentration of both constituents near the bottom increase in the upstream direction in response to decreases in dissolved oxygen. At site $F_{C}$, the concentrations of dissolved iron near the bottom ranged from 0 to $1,100 \mu \mathrm{g} / \mathrm{L}$ and averaged about $250 \mu \mathrm{g} / \mathrm{L}$. The dissolved manganese concentration near the bottom ranged from 0 to $820 \mu \mathrm{g} / \mathrm{L}$ and averaged about $180 \mu \mathrm{g} / \mathrm{L}$.

The average summer concentrations of total inorganic nitrogen varied little throughout the reservoir. The average surface concentration was 0.01 $\mathrm{mg} / \mathrm{L}$ and the average bottom concentration was $0.02 \mathrm{mg} / \mathrm{L}$, except for a sl ight increase at the central site $D_{C}$, to $0.03 \mathrm{mg} / \mathrm{L}$ at the surface and $0.04 \mathrm{mg} / \mathrm{L}$ at the bottom. During the winter the average total inorganic nitrogen concentration was $0.11 \mathrm{mg} / \mathrm{L}$ for surface samples and $0.10 \mathrm{mg} / \mathrm{L}$ for bottom samples at site $A_{C}$ near the dam. The average concentrations were about the same throughout the lake except at site $F_{C}$ where the average concentrations were 0.21 $\mathrm{mg} / \mathrm{L}$ at the surface and $0.25 \mathrm{mg} / \mathrm{L}$ at the bottom.

There was little seasonal variation in the average concentration of total phosphorus. Surface and bottom concentrations, during both summer and winter, averaged about the same, 0.04 and $0.06 \mathrm{mg} / \mathrm{L}$ near the dam, in the central body of the lake, and in the lower tributary arms. At site $F_{C}$, the summer average total phosphorus concentration was $0.09 \mathrm{mg} / \mathrm{L}$ at the surface and $0.11 \mathrm{mg} / \mathrm{L}$ at the bottom, while the winter concentrations were $0.12 \mathrm{mg} / \mathrm{L}$ at the surface and $0.13 \mathrm{mg} / \mathrm{L}$ at the bottom.

Total inorganic nitrogen concentrations fluctuated seasonally, with the highest concentrations during the spring and the lowest concentrations during late summer or early fall. Total phosphorus concentrations usually exhibited no major changes from one season to the next.

There were little seasonal, areal, or depth variations in the concentration of dissolved sol ids, dissolved chloride, dissolved sulfate, or total hardness. The concentrations of these constituents increase during sustained periods of low inflows and decrease during periods of large inflow.

The vol ume-weighted average concentration of dissolved solids ranged from 139 to $292 \mathrm{mg} / \mathrm{L}$ and averaged about $220 \mathrm{mg} / \mathrm{L}$. Vol ume-weighted average concentrations of dissolved chloride ranged from 20 to $68 \mathrm{mg} / \mathrm{L}$ and averaged $43 \mathrm{mg} / \mathrm{L}$. Vol ume-weighted concentrations of dissolved sulfate ranged from 30 to $130 \mathrm{mg} / \mathrm{L}$ and averaged $63 \mathrm{mg} / \mathrm{L}$. Total hardness of the water ranged from 75 to $140 \mathrm{mg} / \mathrm{L}$, expressed as calcium carbonate, placing the water in the moderately hard to hard (61 to $180 \mathrm{mg} / \mathrm{L}$ ) classification. The concentrations of principal dissolved constituents indicate that Somerville Lake is an excellient source of water for municipal, industrial, or agricultural use. 


\section{SELECTED REFERENCES}

Baker, F. E., 1979, Soil survey of Bastrop County, Texas: U.S. Department of Agricul ture Soil Conservation Service.

Britton, L. J., Averett, R. C., and Ferreira, R. F., 1975, An introduction to the processes, problems, and management of urban lakes: U.S. Geological Survey Circular 601-K, 22 p., 6 figs.

Chambers, W. T., 1952, Texas - its land and people: Austin, Texas, Steck Co., $264 \mathrm{p}$.

Dowel1, C. L., and Petty, R. G., 1973, Dams and reservoirs in Texas, part 2: Texas Water Devel opment Board Report 126, $327 \mathrm{p}$.

Fenneman, N. M., 1938, Physiography of eastern United States: New York, McGraw-Hill , 714 p.

Follett, C. R., 1974, Ground-water resources of Brazos and Burleson Counties, Texas: Texas Water Devel opment Board Report 185, 198 p., 27 figs.

Greeson, P. E., 1971, The 1 imnology of Oneida Lake with emphasis on factors contributing to algal blooms: U.S. Geological Survey open-file report, $185 \mathrm{p}$.

Hem, J. D., 1970, Study and interpretation of the chemical characteristics of natural water (2d ed.): U.S. Geological Survey Water-Supply Paper 1473, $363 \mathrm{p.}, 51$ figs.

Ingols, R. S., and Wil roy, R. D., 1963, Mechanism of manganese solution in lake waters: American Water Works Association Journal, v. 55, no. 3, p. 282-290.

Irelan, Burge, and Mendieta, H. B., 1964, Chemical quality of surface waters in the Brazos River basin in Texas: U.S. Geological Survey Water-Supply Paper 1779-K, p. K1-K70, 2 figs., 4 pls.

Kl inkovsky, E., and Moncrief, H., 1961, Generalized soil map of Washington U.S. Department of Agriculture Soil Conservation Service.

Leifeste, D. K., and Popkin, B. P., 1968, Qual ity of water and stratification of Possum Kingdom, Whitney, Hubbard Creek, Proctor, and Belton Reservoirs: Texas Water Development Board Report 85, $122 \mathrm{p}$.

Longwell, C. R., Fl int, F. F., and Sanders, J. E., 1969, Physical Geology: New York, John Wiley, $685 \mathrm{p}$.

Mendieta, H. B., and Bl akey, J. F., 1963, Brazos River basin reservoir studies, progess report, May 1962: Texas Water Commission Memorandum Report 63-01, $24 \mathrm{p} ., 9 \mathrm{pls}$.

Moncrief, H. A., 1960, General ized soil map of Lee County, Texas: U. S. Department of Agriculture Soil Conservation Service.

1960, Generalized soil map of Burleson County, Texas: U.S. Department of Agriculture Soil Conservation Service.

Oborn, E. T., and Hem, J. D., 1962, Some effects of the 1 arger types of aquatic vegetation on iron content of water: U.S. Geological Survey Water-Supply Paper 1459-I, p. I237-I238.

Rawson, Jack, 1967, Study and interpretation of chemical qual ity of surface waters in the Brazos River basin, Texas: Texas Water Development Board Report 55, 118 p., 10 figs.

Sandeen, W. M., 1972, Ground-water resources of Washington County, Texas: Texas Water Devel opment Board Report 162, 105 p., 21 figs.

Texas Department of Health, 1977, Drinking water standards governing water qual ity and reporting requirements for public water supply systems:

Texas Department of Health, Division of Water Hygiene, 17 p. 
Thompson, G. L., 1966, Ground-water resources of Lee County, Texas: Texas Water Development Board Report 20, 131 p., 22 figs.

U.S. Army Corps of Engineers, 1976, Final Environmental Statement for the operations and maintenance programs of Whitney Lake, Waco Lake, Proctor Lake, Still house Hollow Dam and Lake, and Somerville Lake, Brazos River Basin, Texas: Fort Worth District, 64 p., 6 app.

U.S. Envi ronmental Protection Agency, 1976 [1977], Qual ity criteria for water: U.S. Government Printing Office, 256 p.

U.S. Publ ic Health Service, 1962, Drinking Water Standards: U.S. Publ ic Health Service Publication 956, 61 p.

Weast, R. C., 1975, Handbook of chemistry and physics (56th ed.): Cl evel and, Ohio, CRC Press, 2350 p. 


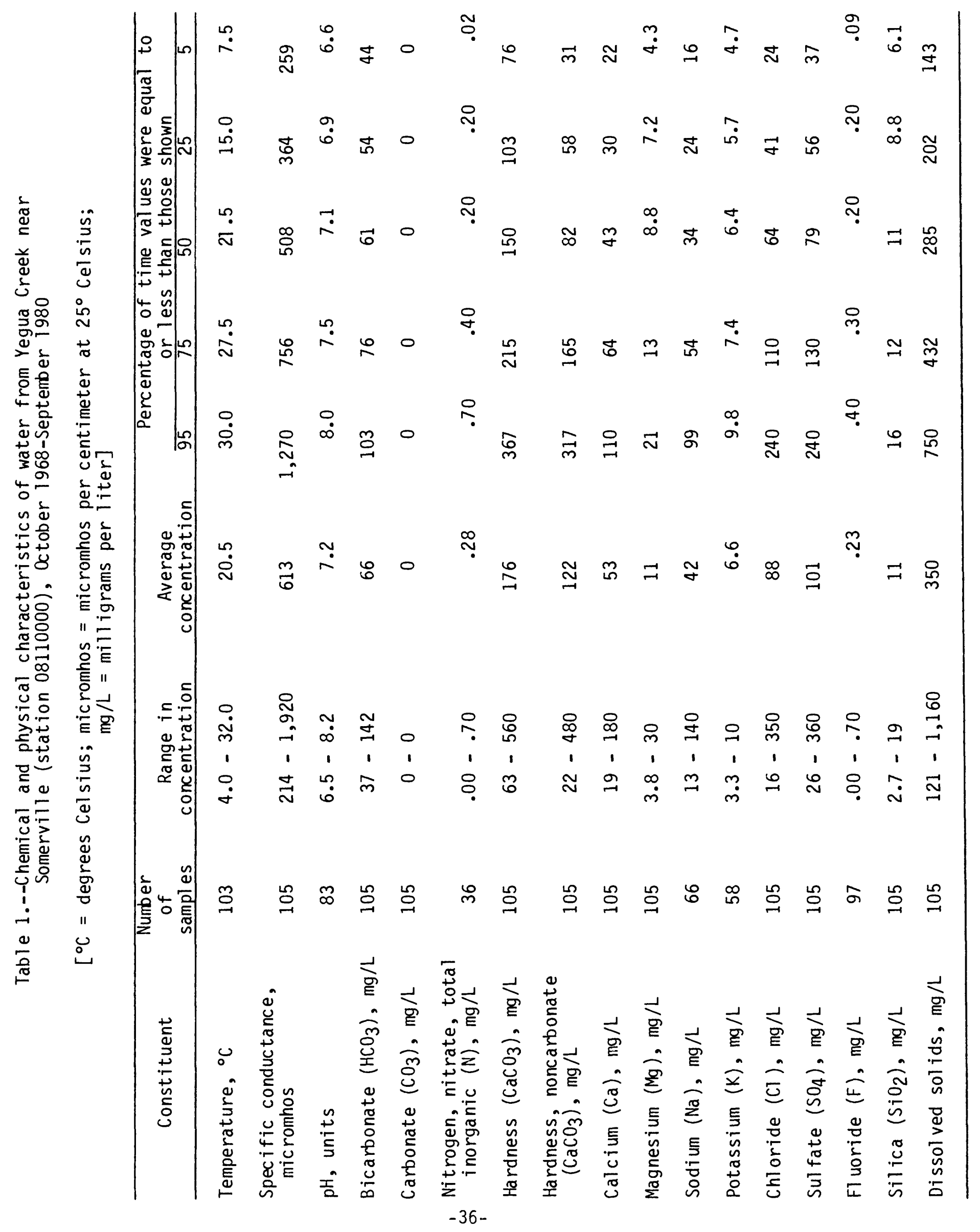


$\mathrm{FT}=$ feet $;$ MICROMHOS = micromhos per centimeter at $25^{\circ} \mathrm{Celsius;}{ }^{\circ} \mathrm{C}-$ degrees Celsius; $\mathrm{MG} / \mathrm{L}=$ milligrams per 1 iter; $\mathrm{UG} / \mathrm{L}=$ micrograms per liter

301908096313101 SOMERVILLE LAKE SITE AC

\begin{tabular}{|c|c|c|c|c|c|c|c|c|c|}
\hline DATE & TIME & $\begin{array}{l}\text { SAMP- } \\
\text { LING } \\
\text { DEPTH } \\
\text { (FT) }\end{array}$ & $\begin{array}{l}\text { SPE- } \\
\text { CIFIC } \\
\text { CON- } \\
\text { DUCT- } \\
\text { ANCE } \\
\text { (MICRO- } \\
\text { MHOS) }\end{array}$ & $\begin{array}{c}\text { PH } \\
\text { FIELD } \\
\text { (UNITS) }\end{array}$ & 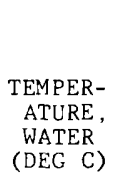 & $\begin{array}{l}\text { TRANS- } \\
\text { PAR- } \\
\text { ENCY } \\
\text { (SECCHI } \\
\text { DISK) } \\
(M)\end{array}$ & $\begin{array}{c}\text { OXYGEN, } \\
\text { DIS- } \\
\text { SOLVED } \\
(M G / L)\end{array}$ & $\begin{array}{c}\text { OXYGEN, } \\
\text { DIS- } \\
\text { SOLVED } \\
\text { (PER- } \\
\text { CENT } \\
\text { SATUR- } \\
\text { ATION) }\end{array}$ & $\begin{array}{l}\text { HARD- } \\
\text { NESS } \\
\text { (MG /L } \\
\text { AS } \\
\text { CACO3) }\end{array}$ \\
\hline $\begin{array}{l}\text { AUG } \\
29 \ldots \\
29 \ldots \\
29 \ldots \\
29 \ldots \\
29 \ldots \\
29 \ldots\end{array}$ & $\begin{array}{l}1120 \\
1122 \\
1124 \\
1126 \\
1128 \\
1130\end{array}$ & $\begin{array}{l}1.0 \\
5.0 \\
10 \\
15 \\
20 \\
28\end{array}$ & $\begin{array}{l}322 \\
322 \\
322 \\
322 \\
322 \\
322\end{array}$ & $\begin{array}{l}7.5 \\
7.2 \\
7.2 \\
7.1 \\
7.1 \\
7.1\end{array}$ & $\begin{array}{l}30.0 \\
29.0 \\
29.0 \\
28.5 \\
28.5 \\
28.5\end{array}$ & $\begin{array}{c}1.10 \\
-- \\
-- \\
-- \\
--\end{array}$ & $\begin{array}{l}5.8 \\
5.8 \\
4.8 \\
4.7 \\
4.4 \\
4.2\end{array}$ & $\begin{array}{l}76 \\
74 \\
62 \\
60 \\
56 \\
54\end{array}$ & $\begin{array}{l}93 \\
-- \\
-- \\
-- \\
-- \\
92\end{array}$ \\
\hline DATE & $\begin{array}{l}\text { HARD- } \\
\text { NESS, } \\
\text { NONCAR- } \\
\text { BONATE } \\
\text { (MG/L } \\
\text { CACO3) }\end{array}$ & $\begin{array}{l}\text { CALCIUM } \\
\text { DIS- } \\
\text { SOLVED } \\
\text { (MG/L } \\
\text { AS CA) }\end{array}$ & $\begin{array}{l}\text { MAGNE- } \\
\text { SIUM, } \\
\text { DIS- } \\
\text { SOLVED } \\
\text { (MG/L } \\
\text { AS MG) }\end{array}$ & $\begin{array}{l}\text { SODIUM, } \\
\text { DIS- } \\
\text { SOLVED } \\
\text { (MG/L } \\
\text { AS NA) }\end{array}$ & $\begin{array}{c}\text { SODIUM } \\
\text { AD- } \\
\text { SORP- } \\
\text { TION } \\
\text { RATIO }\end{array}$ & $\begin{array}{l}\text { POTAS- } \\
\text { SIUM, } \\
\text { DIS- } \\
\text { SOLVED } \\
\text { (MG/L } \\
\text { AS K) }\end{array}$ & $\begin{array}{c}\text { BICAR- } \\
\text { BONATE } \\
\text { (MG / L } \\
\text { AS } \\
\text { HCO3) }\end{array}$ & $\begin{array}{l}\text { SULFATE } \\
\text { DIS- } \\
\text { SOLVED } \\
\text { (MG/L } \\
\text { AS SO4) }\end{array}$ & $\begin{array}{l}\text { CHLO- } \\
\text { RIDE, } \\
\text { DIS- } \\
\text { SOLVED } \\
\text { (MG/L. } \\
\text { AS CL) }\end{array}$ \\
\hline
\end{tabular}

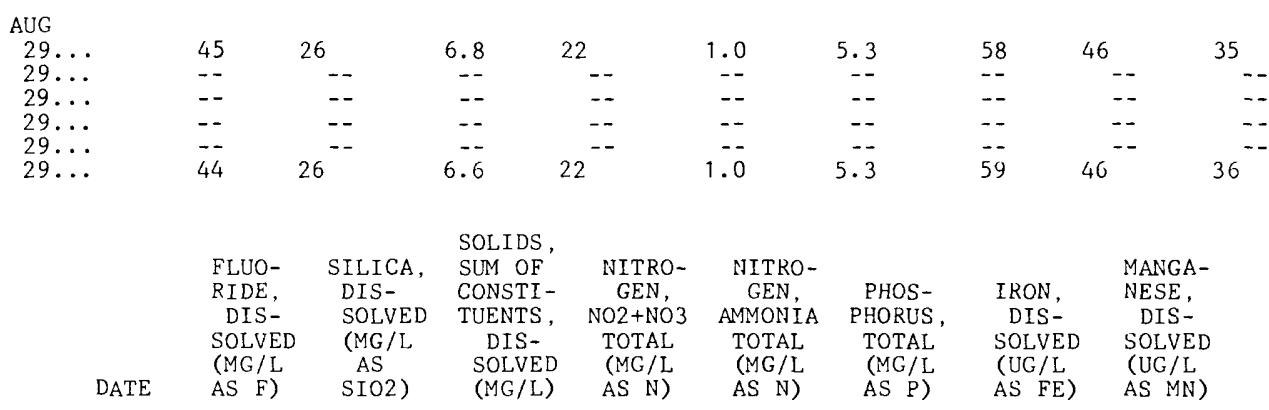

\begin{tabular}{|c|c|c|c|c|c|c|c|c|}
\hline AUG & & & & & & & & \\
\hline $29 \ldots$ & .2 & 9.0 & 179 & .01 & .000 & .010 & 10 & 10 \\
\hline $29 \ldots$ & -- & -- & -- & $\cdots$ & -- & -- & -- & $-\overline{0}$ \\
\hline $29 \ldots$ & -- & - & $\cdots$ & .00 & .000 & .030 & 30 & 0 \\
\hline $29 \ldots$ & -- & -- & -- & - & -- & -- & - & -- \\
\hline $29 \ldots$ & -- & - & -- & -- & -- & -- & - & \\
\hline $29 \ldots$ & .2 & 9.0 & 180 & .01 & .000 & .030 & 230 & 11 \\
\hline
\end{tabular}

301940096315801 SOMERVILLE LAKE SITE AL

\begin{tabular}{|c|c|c|c|c|c|c|c|}
\hline DATE & TIME & $\begin{array}{l}\text { SAMP- } \\
\text { LING } \\
\text { DEPTH } \\
\text { (FT) }\end{array}$ & $\begin{array}{l}\text { SPE- } \\
\text { CIFIC } \\
\text { CON- } \\
\text { DUCT- } \\
\text { ANCE } \\
\text { (MICRO- } \\
\text { MHOS) }\end{array}$ & $\begin{array}{c}\text { PH } \\
\text { FIELD } \\
\text { (UNITS) }\end{array}$ & $\begin{array}{c}\text { TEMPER- } \\
\text { ATURE, } \\
\text { WATER } \\
\text { (DEG C) }\end{array}$ & $\begin{array}{c}\text { OXYGEN, } \\
\text { DIS- } \\
\text { SOLVED } \\
\text { (MG/L) }\end{array}$ & $\begin{array}{c}\text { OXYGEN, } \\
\text { DIS- } \\
\text { SOLVED } \\
\text { (PER- } \\
\text { CENT } \\
\text { SATUR- } \\
\text { ATION) }\end{array}$ \\
\hline \multicolumn{8}{|l|}{ A.UG } \\
\hline $\begin{array}{l}29 \ldots \\
29 \ldots \\
29 \ldots \\
29 \ldots \\
29 \ldots\end{array}$ & $\begin{array}{l}1145 \\
1147 \\
1149 \\
1151 \\
1153\end{array}$ & $\begin{array}{l}1.0 \\
5.0 \\
10 \\
15 \\
24\end{array}$ & $\begin{array}{l}322 \\
322 \\
322 \\
322 \\
322\end{array}$ & $\begin{array}{l}7.6 \\
7.3 \\
7.2 \\
7.1 \\
6.9\end{array}$ & $\begin{array}{l}29.5 \\
29.0 \\
28.5 \\
28.5 \\
28.5\end{array}$ & $\begin{array}{l}5.4 \\
5.3 \\
4.7 \\
4.4 \\
3.6\end{array}$ & $\begin{array}{l}70 \\
68 \\
60 \\
56 \\
46\end{array}$ \\
\hline
\end{tabular}


Table 2--CHEMICAL-QUALITY SURVEYS OF SOMERVILLE LAKE AUGUST 29, 1975--Continued 302026096341501 SOMERVILLE LAKE SITE BC

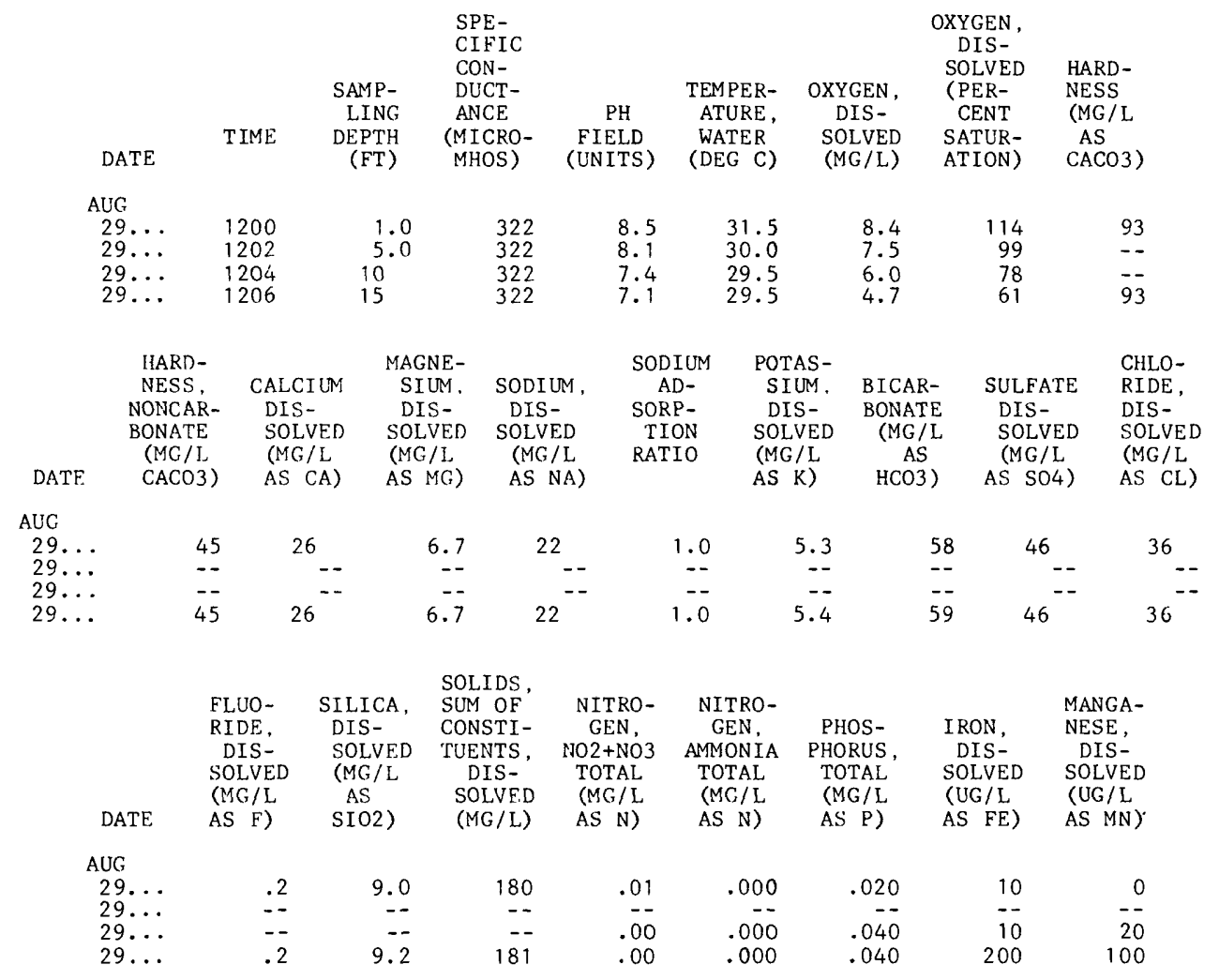

301805096332501 SOMERVILLE LAKE SITE CC

\begin{tabular}{|c|c|c|c|c|c|c|c|c|c|}
\hline DATE & TIME & $\begin{array}{l}\text { SAMP - } \\
\text { LING } \\
\text { DEPTH } \\
(\mathrm{FT})\end{array}$ & $\begin{array}{l}\text { SPE- } \\
\text { CIFIC } \\
\text { CON- } \\
\text { DUCT- } \\
\text { ANCE } \\
\text { (MICRO- } \\
\text { MHOS) }\end{array}$ & $\begin{array}{c}\text { PH } \\
\text { FIELD } \\
\text { (UNITS) }\end{array}$ & $\begin{array}{l}\text { TEMPER- } \\
\text { ATURE, } \\
\text { WATER } \\
(\text { DEG C) }\end{array}$ & $\begin{array}{l}\text { 'IRANS- } \\
\text { PAR- } \\
\text { ENCY } \\
\text { (SECCHI } \\
\text { DISK) } \\
\text { (M) }\end{array}$ & $\begin{array}{c}\text { OXYGEN , } \\
\text { DIS- } \\
\text { SOLVED } \\
\text { (MG/L) }\end{array}$ & $\begin{array}{c}\text { OXYGEN, } \\
\text { DIS- } \\
\text { SOLVED } \\
\text { (PER- } \\
\text { CENT } \\
\text { SATUR- } \\
\text { ATION) }\end{array}$ & $\begin{array}{l}\text { HARD- } \\
\text { NESS } \\
\text { (MG/L } \\
\text { AS } \\
\text { CACO3) }\end{array}$ \\
\hline $\begin{array}{l}\text { AUG } \\
29 \ldots \\
29 \ldots \\
29 \ldots\end{array}$ & $\begin{array}{l}1240 \\
1242 \\
1245\end{array}$ & $\begin{array}{l}1.0 \\
5.0 \\
9.0\end{array}$ & $\begin{array}{l}322 \\
322 \\
322\end{array}$ & $\begin{array}{l}8.5 \\
7.9 \\
7.3\end{array}$ & $\begin{array}{l}30.5 \\
29.0 \\
28.5\end{array}$ & $\begin{array}{l}.80 \\
-- \\
--\end{array}$ & $\begin{array}{l}8.8 \\
7.2 \\
5.7\end{array}$ & $\begin{array}{r}116 \\
92 \\
73\end{array}$ & $\begin{array}{l}93 \\
-- \\
91\end{array}$ \\
\hline DATE. & $\begin{array}{l}\text { HARD- } \\
\text { NESS } \\
\text { NONCAR- } \\
\text { BONATE } \\
\text { (MG/L } \\
\text { CACO3) }\end{array}$ & $\begin{array}{l}\text { CALCIUM } \\
\text { DIS- } \\
\text { SOLVED } \\
\text { (MG/L } \\
\text { AS CA) }\end{array}$ & $\begin{array}{l}\text { MAGNE- } \\
\text { SIUM , } \\
\text { DIS- } \\
\text { SOLVED } \\
\text { (MG/L } \\
\text { AS MG) }\end{array}$ & $\begin{array}{l}\text { SODIUM, } \\
\text { DIS- } \\
\text { SOLVED } \\
\text { (MG/L } \\
\text { AS NA) }\end{array}$ & $\begin{array}{c}\text { SODIUM } \\
\text { AD- } \\
\text { SORP- } \\
\text { TION } \\
\text { RATIO }\end{array}$ & $\begin{array}{l}\text { POTAS- } \\
\text { SIUM, } \\
\text { DIS- } \\
\text { SOLVED } \\
(M G / L \\
\text { AS K) }\end{array}$ & $\begin{array}{c}\text { BICAR- } \\
\text { BONATE } \\
\text { (MG / L } \\
\text { AS } \\
\text { HCO3) }\end{array}$ & $\begin{array}{l}\text { SULFATE } \\
\text { DIS- } \\
\text { SOLVED } \\
\text { (MG/L } \\
\text { AS SO4) }\end{array}$ & $\begin{array}{l}\text { CHLO- } \\
\text { RIDE, } \\
\text { DIS- } \\
\text { SOLVED } \\
\text { (MG/L } \\
\text { AS CL) }\end{array}$ \\
\hline
\end{tabular}

\begin{tabular}{|c|c|c|c|c|c|c|c|c|c|}
\hline AUG & & & & & & & & & \\
\hline $29 \ldots$ & 44 & 26 & 6.9 & 22 & 1.0 & 5.3 & 60 & 46 & 35 \\
\hline $29 \ldots$ & -- & $26^{--}$ & $6 .-$ & $22^{--}$ & 1.0 & $5 . \overline{3}$ & $-\overline{62}$ & $46^{--}$ & 35 \\
\hline
\end{tabular}


Table 2--CHEMICAL-QUALITY SURVEYS OF SOMERVILLE LAKE AUGUST 29, 1975--Continued

301805096332501 SOMERVILLE LAKE SITE CC--Continued

\begin{tabular}{|c|c|c|c|c|c|c|c|c|}
\hline & $\begin{array}{l}\text { FLUO- } \\
\text { RIDE, } \\
\text { DIS- } \\
\text { SOLVED } \\
\text { (MG/L }\end{array}$ & $\begin{array}{l}\text { SILICA, } \\
\text { DIS- } \\
\text { SOLVED } \\
\text { (MG/L } \\
\text { AS }\end{array}$ & $\begin{array}{l}\text { SOLIDS, } \\
\text { SUM OF } \\
\text { CONSTI- } \\
\text { TUENTS, } \\
\text { DIS- } \\
\text { SOLVED }\end{array}$ & $\begin{array}{c}\text { NITRO- } \\
\text { GEN, } \\
\text { NO2+NO3 } \\
\text { TOTAL } \\
\text { (MG/L }\end{array}$ & $\begin{array}{l}\text { NITRO- } \\
\text { GEN, } \\
\text { AMMONIA } \\
\text { TOTAL } \\
\text { (MG/L }\end{array}$ & $\begin{array}{c}\text { PHOS- } \\
\text { PHORUS, } \\
\text { TOTAL } \\
\text { (MG/L }\end{array}$ & $\begin{array}{l}\text { IRON, } \\
\text { DIS- } \\
\text { SOLVED } \\
\text { (UG / L }\end{array}$ & $\begin{array}{l}\text { MANGA- } \\
\text { NESE, } \\
\text { DIS- } \\
\text { SOLVED } \\
\text { (UG / L }\end{array}$ \\
\hline & & & & & & & & \\
\hline
\end{tabular}

AUG

\begin{tabular}{|c|c|c|c|c|c|c|c|c|}
\hline $29 \ldots$ & .2 & 9.3 & 181 & .00 & .000 & .060 & 30 & 0 \\
\hline $29 \ldots$ & -- & -- & $=-$ & -- & -- & -- & -- & -- \\
\hline$\ldots$ & .2 & 9.6 & 181 & .00 & .000 & .070 & 40 & 0 \\
\hline
\end{tabular}

301904096335601 SOMERVILLE LAKE SITE DC

\begin{tabular}{|c|c|c|c|c|c|c|c|}
\hline DATE & TIME & $\begin{array}{l}\text { SAMP - } \\
\text { LING } \\
\text { DEPTH } \\
(\mathrm{FT})\end{array}$ & $\begin{array}{l}\text { SPE- } \\
\text { CIFIC } \\
\text { CON- } \\
\text { DUCT- } \\
\text { ANCE } \\
\text { (MICRO- } \\
\text { MHOS) }\end{array}$ & $\begin{array}{c}\text { PH } \\
\text { FIELD } \\
\text { (UNITS) }\end{array}$ & $\begin{array}{l}\text { TEMPER- } \\
\text { ATURE, } \\
\text { WATER } \\
\text { (DEG C) }\end{array}$ & $\begin{array}{c}\text { OXYGEN, } \\
\text { DIS- } \\
\text { SOLVED } \\
(M G / L)\end{array}$ & $\begin{array}{c}\text { OXYGEN, } \\
\text { DIS- } \\
\text { SOLVED } \\
\text { (PER- } \\
\text { CENT } \\
\text { SATUR- } \\
\text { ATION) }\end{array}$ \\
\hline \multicolumn{8}{|l|}{ AUG } \\
\hline $\begin{array}{l}29 \ldots \\
29 \ldots \\
29 \ldots \\
29 \ldots \\
29 \ldots\end{array}$ & $\begin{array}{l}1300 \\
1302 \\
1304 \\
1307 \\
1310\end{array}$ & $\begin{array}{l}1.0 \\
5.0 \\
10 \\
15 \\
24\end{array}$ & $\begin{array}{l}322 \\
322 \\
322 \\
322 \\
322\end{array}$ & $\begin{array}{l}8.7 \\
8.4 \\
7.5 \\
7.3 \\
7.0\end{array}$ & $\begin{array}{l}30.5 \\
29.5 \\
29.0 \\
29.0 \\
29.0\end{array}$ & $\begin{array}{l}9.2 \\
8.1 \\
5.8 \\
5.1 \\
3.6\end{array}$ & $\begin{array}{r}121 \\
105 \\
74 \\
65 \\
46\end{array}$ \\
\hline
\end{tabular}

301754096380801 SOMERVILLE LAKE SITE FC

\begin{tabular}{|c|c|c|c|c|c|c|c|c|c|}
\hline DATE & TIME & $\begin{array}{l}\text { SAMP- } \\
\text { LING } \\
\text { DEPTH } \\
\text { (FT) }\end{array}$ & $\begin{array}{l}\text { SPE- } \\
\text { CIFIC } \\
\text { CON- } \\
\text { DUCT- } \\
\text { ANCE } \\
\text { (MICRO- } \\
\text { MHOS) }\end{array}$ & $\begin{array}{c}\text { PH } \\
\text { FIELD } \\
\text { (UNITS) }\end{array}$ & $\begin{array}{l}\text { TEMPER- } \\
\text { ATURE, } \\
\text { WATER } \\
\text { (DEG C) }\end{array}$ & $\begin{array}{l}\text { TRANS- } \\
\text { PAR- } \\
\text { ENCY } \\
\text { (SECCHI } \\
\text { DISK) } \\
\text { (M) }\end{array}$ & $\begin{array}{c}\text { OXYGEN, } \\
\text { DIS- } \\
\text { SOLVED } \\
\text { (MG/L) }\end{array}$ & $\begin{array}{c}\text { OXYGEN, } \\
\text { DIS- } \\
\text { SOLVED } \\
\text { (PER- } \\
\text { CENT } \\
\text { SATUR- } \\
\text { ATION) }\end{array}$ & $\begin{array}{l}\text { HARD - } \\
\text { NESS } \\
(\mathrm{MG} / \mathrm{L} \\
\text { AS } \\
\text { CACO3) }\end{array}$ \\
\hline $\begin{array}{l}\text { UUC } \\
29 \ldots \\
29 \ldots \\
29 \ldots \\
29 \ldots\end{array}$ & $\begin{array}{l}1325 \\
1327 \\
1329 \\
1333\end{array}$ & $\begin{array}{l}1.0 \\
5.0 \\
10 \\
15\end{array}$ & $\begin{array}{l}365 \\
399 \\
399 \\
399\end{array}$ & $\begin{array}{l}9.0 \\
7.4 \\
7.1 \\
6.9\end{array}$ & $\begin{array}{l}30.5 \\
29.0 \\
28.5 \\
28.5\end{array}$ & $\begin{array}{c}50 \\
-- \\
-- \\
--\end{array}$ & $\begin{array}{r}12.0 \\
5.4 \\
2.8 \\
1.7\end{array}$ & $\begin{array}{r}158 \\
69 \\
36 \\
22\end{array}$ & $\begin{array}{l}100 \\
- \\
110\end{array}$ \\
\hline DATE & $\begin{array}{l}\text { HARD- } \\
\text { NES S } \\
\text { NONCAR- } \\
\text { BONATE } \\
\text { (MG/L } \\
\text { CACO3) }\end{array}$ & $\begin{array}{l}\text { CALCIUM } \\
\text { DIS- } \\
\text { SOLVED } \\
\text { (MG/L } \\
\text { AS CA) }\end{array}$ & $\begin{array}{l}\text { MAGNE- } \\
\text { SIUM, } \\
\text { DIS- } \\
\text { SOLVED } \\
\text { (MG/L } \\
\text { AS MG) }\end{array}$ & $\begin{array}{l}\text { SODIUM, } \\
\text { DIS- } \\
\text { SOLVED } \\
\text { (MG/L } \\
\text { AS NA) }\end{array}$ & $\begin{array}{c}\text { SODIUM } \\
\text { AD- } \\
\text { SORP- } \\
\text { TION } \\
\text { RATIO }\end{array}$ & $\begin{array}{l}\text { POTAS- } \\
\text { SIUM, } \\
\text { DIS- } \\
\text { SOLVED } \\
\text { (MG/L } \\
\text { AS K) }\end{array}$ & $\begin{array}{c}\text { BICAR- } \\
\text { BONATE } \\
\text { (MG / L } \\
\text { AS } \\
\text { HCO3) }\end{array}$ & $\begin{array}{l}\text { SULFATE } \\
\text { DIS- } \\
\text { SOLVED } \\
\text { (MG/L } \\
\text { AS SO4) }\end{array}$ & $\begin{array}{l}\text { CHLO- } \\
\text { RIDE, } \\
\text { DIS- } \\
\text { SOLVED } \\
\text { (MG/L } \\
\text { AS CL) }\end{array}$ \\
\hline
\end{tabular}

\begin{tabular}{|c|c|c|c|c|c|c|c|c|c|}
\hline $\begin{array}{r}A \cup G \\
29\end{array}$ & 56 & & & & & & & & \\
\hline $29 \ldots$ & $\ldots$ & .. & 14 & 20 & 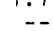 & 3.2 & I & -. & 42 \\
\hline $29 \ldots$ & -- & -- & -- & -- & - & -- & -- & -. & $\ldots$ \\
\hline $29 \ldots$ & 63 & 31 & 8.3 & 29 & 1.2 & 5.8 & 59 & 63 & 47 \\
\hline
\end{tabular}

\begin{tabular}{|c|c|c|c|c|c|c|c|c|}
\hline DATE & $\begin{array}{l}\text { FLUO- } \\
\text { RIDE, } \\
\text { DIS- } \\
\text { SOLVED } \\
\text { (MG/L } \\
\text { AS F) }\end{array}$ & $\begin{array}{l}\text { SILICA, } \\
\text { DIS- } \\
\text { SOLVED } \\
\text { (MG/L } \\
\text { AS } \\
\text { SIO2) }\end{array}$ & $\begin{array}{l}\text { SOLIDS, } \\
\text { SUM OF } \\
\text { CONSTI- } \\
\text { TUENTS, } \\
\text { DIS- } \\
\text { SOLVED } \\
(\mathrm{MG} / \mathrm{L})\end{array}$ & $\begin{array}{c}\text { NITRO- } \\
\text { GEN, } \\
\text { NO2+NO3 } \\
\text { TOTAL } \\
\text { (MC/L } \\
\text { AS N) }\end{array}$ & $\begin{array}{l}\text { NITRO- } \\
\text { GEN, } \\
\text { AMMONIA } \\
\text { TOTAL } \\
\text { (MG/L } \\
\text { AS N) }\end{array}$ & $\begin{array}{l}\text { PHOS- } \\
\text { PHORUS, } \\
\text { TOTAL } \\
\text { (MG/L } \\
\text { AS P) }\end{array}$ & $\begin{array}{l}\text { IRON, } \\
\text { DIS- } \\
\text { SOLVED } \\
\text { (UG/L } \\
\text { AS FE) }\end{array}$ & $\begin{array}{l}\text { MANGA- } \\
\text { NESE, } \\
\text { DIS- } \\
\text { SOLVED } \\
\text { (UG / L } \\
\text { AS MN) }\end{array}$ \\
\hline \multicolumn{9}{|l|}{ AUG } \\
\hline $29 \ldots$ & .2 & 11 & 204 & .01 & .000 & .070 & 10 & 10 \\
\hline $\begin{array}{l}29 \ldots \ldots \\
29 \ldots\end{array}$ & -- & -- & -- & $\overline{0}$ & סי & .080 & 40 & $3 \overline{0}$ \\
\hline $29 \ldots$ & .2 & 11 & 226 & .00 & .000 & .0140 & 790 & 820 \\
\hline
\end{tabular}


$\mathrm{FT}=$ feet; MICROMHOS = micromhos per centimeter at $25^{\circ}$ Celsius; ${ }^{\circ} \mathrm{C}$ - degrees Celsius; $\mathrm{MG} / \mathrm{L}=$ milligrams per liter; $\mathrm{UC} / \mathrm{L}=$ micrograms per liter

301908096313101 SOMERVILLE LAKE SITE AC

\begin{tabular}{|c|c|c|c|c|c|c|c|c|c|}
\hline DATE & TIME & $\begin{array}{l}\text { SAMP- } \\
\text { LING } \\
\text { DEPTH } \\
(\mathrm{FT})\end{array}$ & $\begin{array}{l}\text { SPE- } \\
\text { CIFIC } \\
\text { CON- } \\
\text { DUCT- } \\
\text { ANCE } \\
\text { (MICRO- } \\
\text { MHOS) }\end{array}$ & $\begin{array}{c}\text { PH } \\
\text { FIELD } \\
\text { (UNITS) }\end{array}$ & $\begin{array}{c}\text { TEMPER- } \\
\text { ATURE } \\
\text { WATER } \\
\text { (DEG C) }\end{array}$ & $\begin{array}{l}\text { TRANS- } \\
\text { PAR- } \\
\text { ENCY } \\
\text { (SECCHI } \\
\text { DISK) } \\
\text { (M) }\end{array}$ & $\begin{array}{c}\text { OXYGEN, } \\
\text { DIS- } \\
\text { SOLVED } \\
\text { (MG/L) }\end{array}$ & $\begin{array}{c}\text { OXYGEN, } \\
\text { DIS- } \\
\text { SOLVED } \\
\text { (PER- } \\
\text { CENT } \\
\text { SATUR- } \\
\text { ATION) }\end{array}$ & $\begin{array}{l}\text { HARD- } \\
\text { NESS } \\
\text { (MG/L } \\
\text { AS } \\
\text { CACO3) }\end{array}$ \\
\hline $\begin{array}{c}\text { FEB } \\
03 \ldots \\
03 \ldots \\
03 \ldots \\
03 \ldots\end{array}$ & $\begin{array}{l}1430 \\
1432 \\
1434 \\
1436\end{array}$ & $\begin{array}{l}1.0 \\
10 \\
20 \\
27\end{array}$ & $\begin{array}{l}369 \\
369 \\
369 \\
369\end{array}$ & $\begin{array}{l}7.9 \\
7.9 \\
7.9 \\
7.9\end{array}$ & $\begin{array}{l}12.5 \\
12.5 \\
12.5 \\
12.5\end{array}$ & $\begin{array}{c}.52 \\
-- \\
-- \\
--\end{array}$ & $\begin{array}{l}10.3 \\
10.1 \\
10.1 \\
10.0\end{array}$ & $\begin{array}{l}96 \\
94 \\
94 \\
93\end{array}$ & $\begin{array}{r}110 \\
-- \\
110\end{array}$ \\
\hline DATE & $\begin{array}{l}\text { HARD- } \\
\text { NESS } \\
\text { NONCAR- } \\
\text { BONATE } \\
\text { (MG / L } \\
\text { CACO3) }\end{array}$ & $\begin{array}{l}\text { CALCIUM } \\
\text { DIS- } \\
\text { SOLVED } \\
\text { (MG/L } \\
\text { AS CA) }\end{array}$ & $\begin{array}{l}\text { MAGNE- } \\
\text { SIUM, } \\
\text { DIS- } \\
\text { SOLVED } \\
\text { (MG/L } \\
\text { AS MG) }\end{array}$ & $\begin{array}{l}\text { SODIUM, } \\
\text { DIS- } \\
\text { SOLVED } \\
\text { (MG/L } \\
\text { AS NA) }\end{array}$ & $\begin{array}{c}\text { SODIUM } \\
\text { AD- } \\
\text { SORP- } \\
\text { TION } \\
\text { RATIO }\end{array}$ & $\begin{array}{l}\text { POTAS- } \\
\text { SIUM, } \\
\text { DIS- } \\
\text { SOLVED } \\
\text { (MG/L } \\
\text { AS K) }\end{array}$ & $\begin{array}{c}\text { BICAR- } \\
\text { BONATE } \\
(\mathrm{MG} / \mathrm{L} \\
\text { AS } \\
\mathrm{HCO} 3)\end{array}$ & $\begin{array}{l}\text { SULFATE } \\
\text { DIS- } \\
\text { SOLVED } \\
\text { (MG/L } \\
\text { AS SO4) }\end{array}$ & $\begin{array}{l}\text { CHLO- } \\
\text { RIDE, } \\
\text { DIS- } \\
\text { SOLVED } \\
\text { (MG/L } \\
\text { AS CL) }\end{array}$ \\
\hline
\end{tabular}

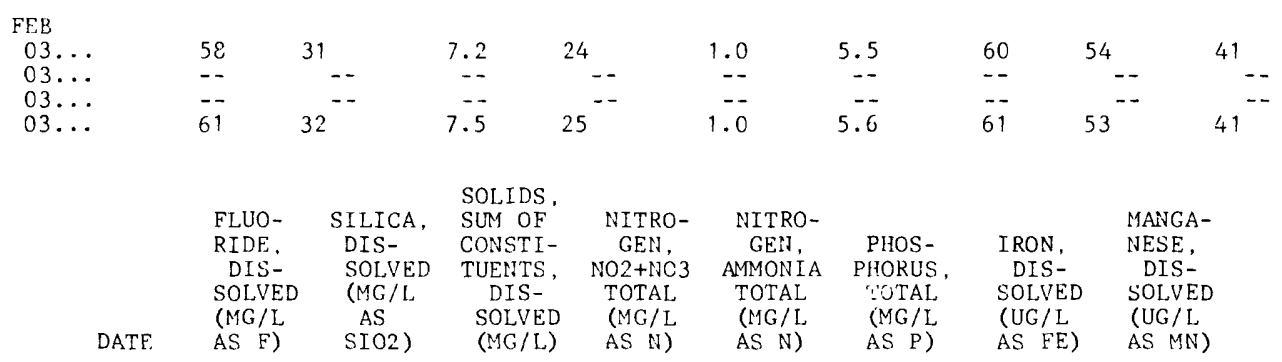

\begin{tabular}{|c|c|c|c|c|c|c|c|c|}
\hline \multicolumn{9}{|l|}{ FEB } \\
\hline $\begin{array}{l}03 \ldots \\
03 \ldots\end{array}$ & $\begin{array}{l}.2 \\
--\end{array}$ & $\begin{array}{r}9.9 \\
--\end{array}$ & $\begin{array}{r}203 \\
-\end{array}$ & $\begin{array}{l}.00 \\
.00\end{array}$ & $\begin{array}{r}.010 \\
.010\end{array}$ & $\begin{array}{l}.040 \\
.030\end{array}$ & $\begin{array}{r}170 \\
60\end{array}$ & $\begin{array}{r}140 \\
60\end{array}$ \\
\hline $03 \ldots$ & - & - & -. & - & - & -. & -. & $\ldots$ \\
\hline $03 \ldots$ & .2 & 9.9 & 204 & .00 & .000 & .040 & 0 & 0 \\
\hline
\end{tabular}

301940096315801 SOMERVILLE LAKE SITE AL

\begin{tabular}{|c|c|c|c|c|c|c|c|}
\hline DATE & TIME & $\begin{array}{c}\text { SAMP- } \\
\text { LING } \\
\text { DEPTH } \\
(F T)\end{array}$ & $\begin{array}{l}\text { SPE- } \\
\text { CIFIC } \\
\text { CON- } \\
\text { DUCT- } \\
\text { ANCE } \\
\text { (MICRO- } \\
\text { MHOS) }\end{array}$ & $\begin{array}{c}\text { PH } \\
\text { FIELD } \\
\text { (UNITS) }\end{array}$ & $\begin{array}{c}\text { TEMPER- } \\
\text { ATURE, } \\
\text { WATER } \\
\text { (DEG C) }\end{array}$ & $\begin{array}{c}\text { OXYGEN, } \\
\text { DIS- } \\
\text { SOLVED } \\
(\mathrm{MG} / \mathrm{L})\end{array}$ & $\begin{array}{c}\text { OXYGEN, } \\
\text { DIS- } \\
\text { SOLVED } \\
\text { (PER- } \\
\text { CENT } \\
\text { SATUR- } \\
\text { ATION) }\end{array}$ \\
\hline \multicolumn{8}{|l|}{ FEB } \\
\hline $\begin{array}{l}03 \ldots \\
03 \ldots \\
03 \ldots\end{array}$ & $\begin{array}{l}1450 \\
1452 \\
1454\end{array}$ & $\begin{array}{l}1.0 \\
10 \\
24\end{array}$ & $\begin{array}{l}369 \\
369 \\
369\end{array}$ & $\begin{array}{l}7.8 \\
7.8 \\
7.9\end{array}$ & $\begin{array}{l}12.5 \\
12.0 \\
12.0\end{array}$ & $\begin{array}{l}10.4 \\
10.3 \\
10.2\end{array}$ & $\begin{array}{l}97 \\
95 \\
94\end{array}$ \\
\hline
\end{tabular}

302026096341501 SOMERVILLE LAKE SITE BC

\begin{tabular}{|c|c|c|c|c|c|c|c|}
\hline DATE & TIME & $\begin{array}{l}\text { SAMP- } \\
\text { LING } \\
\text { DEPTH } \\
(F T)\end{array}$ & $\begin{array}{l}\text { SPE- } \\
\text { CIFIC } \\
\text { CON- } \\
\text { DUCT- } \\
\text { ANCE } \\
\text { (MICRO- } \\
\text { MHOS) }\end{array}$ & $\begin{array}{c}\text { PH } \\
\text { FIELD } \\
\text { (UNITS) }\end{array}$ & $\begin{array}{l}\text { TEMPER- } \\
\text { ATURE, } \\
\text { WATER } \\
\text { (DEG C) }\end{array}$ & $\begin{array}{c}\text { OXYGEN, } \\
\text { DIS- } \\
\text { SOLVED } \\
\text { (MG/L) }\end{array}$ & $\begin{array}{c}\text { OXYGEN, } \\
\text { DIS- } \\
\text { SOLVED } \\
\text { (PER- } \\
\text { CENT } \\
\text { SATUR- } \\
\text { ATION) }\end{array}$ \\
\hline $\begin{array}{l}\text { FEB } \\
03 \ldots \\
03 \ldots\end{array}$ & $\begin{array}{l}1500 \\
1502\end{array}$ & $13^{1.0}$ & $\begin{array}{l}369 \\
369\end{array}$ & $\begin{array}{l}7.9 \\
7.9\end{array}$ & $\begin{array}{l}12.0 \\
12.0\end{array}$ & $\begin{array}{l}10.3 \\
10.1\end{array}$ & $\begin{array}{l}95 \\
94\end{array}$ \\
\hline
\end{tabular}


Table 3--CHEMICAL-QUALITY SURVEYS OF SOMERVILLE LAKE FEBRUARY 3, 1976--Continued

301805096332501 SOMERVILLE LAKE SITE CC

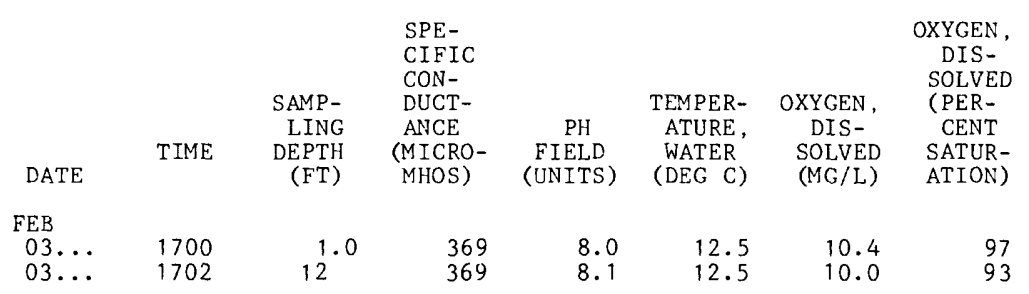

$30184709633460^{7}$ SOMERVILLE LAKE SITE DR

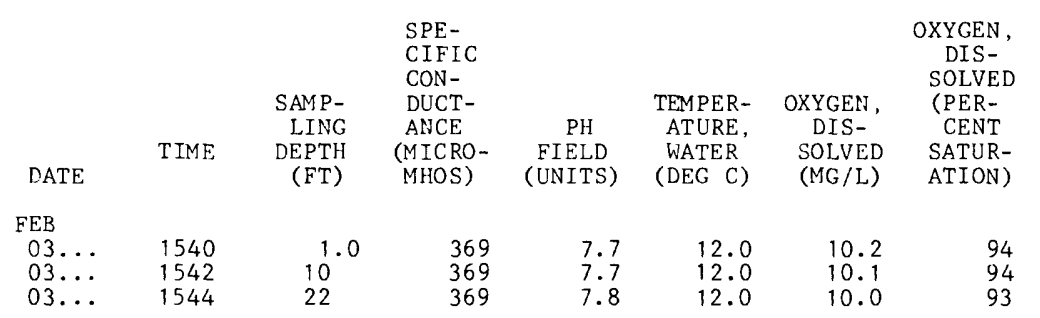

301904096335601 SOMERVILLE LAKE SITE DC

\begin{tabular}{|c|c|c|c|c|c|c|c|c|c|}
\hline DATE & TIMF & $\begin{array}{l}\text { SAMP- } \\
\text { LING } \\
\text { DEPTH } \\
\text { (FT) }\end{array}$ & $\begin{array}{l}\text { SPE- } \\
\text { CIFIC } \\
\text { CON- } \\
\text { DUCT- } \\
\text { ANCE } \\
\text { (MICRO- } \\
\text { MHOS) }\end{array}$ & $\begin{array}{c}\text { PH } \\
\text { FIELD } \\
\text { (UNITS) }\end{array}$ & 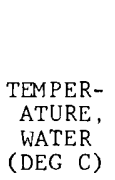 & $\begin{array}{l}\text { TRANS- } \\
\text { PAR- } \\
\text { ENCY } \\
\text { (SECCHI } \\
\text { DISK) } \\
\text { (M) }\end{array}$ & $\begin{array}{c}\text { OXYGEN, } \\
\text { DIS- } \\
\text { SOLVED } \\
\text { (MG/L) }\end{array}$ & $\begin{array}{c}\text { OXYGEN, } \\
\text { DIS- } \\
\text { SOLVED } \\
\text { (PER- } \\
\text { CENT } \\
\text { SATUR- } \\
\text { ATION) }\end{array}$ & $\begin{array}{l}\text { HARD- } \\
\text { NESS } \\
(\mathrm{MG} / \mathrm{L} \\
\mathrm{AS} \\
\mathrm{CACO} 3)\end{array}$ \\
\hline $\begin{array}{l}\text { EB } \\
03 \ldots \\
03 \ldots \\
03 \ldots \\
03 \ldots\end{array}$ & $\begin{array}{l}1520 \\
1522 \\
1524 \\
1526\end{array}$ & $\begin{array}{l}1.0 \\
10 \\
20 \\
24\end{array}$ & $\begin{array}{l}369 \\
369 \\
369 \\
369\end{array}$ & $\begin{array}{l}7.8 \\
7.7 \\
7.7 \\
7.7\end{array}$ & $\begin{array}{l}12.0 \\
12.0 \\
12.0 \\
12.0\end{array}$ & $\begin{array}{c}.61 \\
-- \\
-- \\
--\end{array}$ & $\begin{array}{r}10.3 \\
10.3 \\
10.2 \\
9.8\end{array}$ & $\begin{array}{l}95 \\
95 \\
94 \\
91\end{array}$ & $\begin{array}{l}110 \\
-- \\
110\end{array}$ \\
\hline DATE & $\begin{array}{l}\text { HARD- } \\
\text { NESS } \\
\text { NONCAR- } \\
\text { BONATE } \\
\text { (MG/L } \\
\text { CACO3) }\end{array}$ & $\begin{array}{l}\text { CALCI UM } \\
\text { DIS- } \\
\text { SOLVED } \\
\text { (MG/L } \\
\text { AS CA) }\end{array}$ & $\begin{array}{l}\text { MAGNE- } \\
\text { SIUM, } \\
\text { DIS- } \\
\text { SOLVED } \\
\text { (MG/L } \\
\text { AS MG) }\end{array}$ & $\begin{array}{l}\text { SODIUM, } \\
\text { DIS- } \\
\text { SOLVED } \\
\text { (MG/L } \\
\text { AS NA) }\end{array}$ & $\begin{array}{c}\text { SODIUM } \\
\text { AD- } \\
\text { SORP- } \\
\text { TION } \\
\text { PATIO }\end{array}$ & $\begin{array}{l}\text { POTAS- } \\
\text { SIUM, } \\
\text { DIS- } \\
\text { SOLVED } \\
\text { (MG/L } \\
\text { AS K) }\end{array}$ & $\begin{array}{c}\text { BICAR- } \\
\text { BONATE } \\
\text { (MG/L } \\
\text { AS } \\
\text { HCO3) }\end{array}$ & $\begin{array}{l}\text { SULFATE } \\
\text { DIS- } \\
\text { SOLVED } \\
\text { (MG/L } \\
\text { AS SO4) }\end{array}$ & $\begin{array}{l}\text { CHLO- } \\
\text { RIDE, } \\
\text { DIS- } \\
\text { SOLVED } \\
\text { (MG/L } \\
\text { AS CL) }\end{array}$ \\
\hline
\end{tabular}

\begin{tabular}{|c|c|c|c|c|c|c|c|c|c|}
\hline $\begin{array}{l}\mathrm{FEB} \\
03 \ldots\end{array}$ & 58 & 31 & 7.3 & 24 & 1.0 & 5.5 & 60 & 52 & 41 \\
\hline $03 \ldots$ & -- & -- & -- & -- & - & -- & -- & -- & -- \\
\hline $03 \ldots$ & -- & -- & -- & -- & -- & -- & -- & -- & - \\
\hline $03 \ldots$ & 56 & 31 & 7.0 & 24 & 1.0 & 5.5 & 61 & 54 & 42 \\
\hline
\end{tabular}

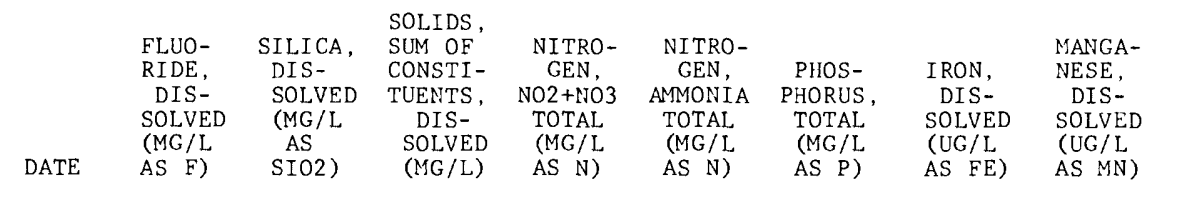

\begin{tabular}{|c|c|c|c|c|c|c|c|c|}
\hline FEB & & & & & & & & \\
\hline $03 \ldots$ & .2 & 10 & 201 & .00 & .010 & .030 & 20 & 0 \\
\hline $03 \ldots$ & -- & -- & -- & .00 & .010 & .040 & 0 & 0 \\
\hline $03 \ldots$ & -- & -- & -- & -- & $\cdots$ & -- & -- & -- \\
\hline $03 \ldots$ & .2 & 9.7 & 203 & .01 & .010 & .040 & 0 & 0 \\
\hline
\end{tabular}


301817096364101 SOMERVILLE LAKE SITE EC

\begin{tabular}{|c|c|c|c|c|c|c|c|c|c|}
\hline DATE & TIME & $\begin{array}{l}\text { SAMP- } \\
\text { LING } \\
\text { DEPTH } \\
\text { (FT) }\end{array}$ & $\begin{array}{l}\text { SPE- } \\
\text { CIFIC } \\
\text { CON- } \\
\text { DUCT- } \\
\text { ANCE } \\
\text { (MICRO- } \\
\text { MHOS) }\end{array}$ & $\begin{array}{c}\text { PH } \\
\text { FIELD } \\
\text { (UNITS) }\end{array}$ & $\begin{array}{l}\text { TEMPER- } \\
\text { ATURE, } \\
\text { WATER } \\
\text { (DEG C) }\end{array}$ & $\begin{array}{l}\text { TRANS- } \\
\text { PAR- } \\
\text { ENCY } \\
\text { (SECCHI } \\
\text { DISK) } \\
\text { (M) }\end{array}$ & $\begin{array}{c}\text { OXYGEN, } \\
\text { DIS- } \\
\text { SOLVED } \\
\text { (MG/L) }\end{array}$ & $\begin{array}{c}\text { OXYGEN, } \\
\text { DIS- } \\
\text { SOLVED } \\
\text { (PER- } \\
\text { CENT } \\
\text { SATUR- } \\
\text { ATION) }\end{array}$ & $\begin{array}{l}\text { HARD- } \\
\text { NESS } \\
\text { (MG/L } \\
\text { AS } \\
\text { CACO3) }\end{array}$ \\
\hline $\begin{array}{l}\text { FEB } \\
03 \ldots \\
03 \ldots \\
03 \ldots\end{array}$ & $\begin{array}{l}1600 \\
1602 \\
1604\end{array}$ & $\begin{array}{l}1.0 \\
10 \\
19\end{array}$ & $\begin{array}{l}370 \\
370 \\
370\end{array}$ & $\begin{array}{l}7.7 \\
7.7 \\
7.7\end{array}$ & $\begin{array}{l}12.0 \\
12.0 \\
12.0\end{array}$ & $\begin{array}{l}.61 \\
-- \\
-.\end{array}$ & $\begin{array}{l}10.3 \\
10.3 \\
10.3\end{array}$ & $\begin{array}{l}95 \\
95 \\
95\end{array}$ & $\begin{array}{l}110 \\
110\end{array}$ \\
\hline DATE & $\begin{array}{l}\text { HARD- } \\
\text { NESS } \\
\text { NONCAR- } \\
\text { BONATE } \\
\text { (MG/L } \\
\text { CACO3) }\end{array}$ & $\begin{array}{l}\text { CALCIUM } \\
\text { DIS- } \\
\text { SOLVED } \\
\text { (MG/L } \\
\text { AS CA) }\end{array}$ & $\begin{array}{l}\text { MAGNE- } \\
\text { SIUM } \\
\text { DIS- } \\
\text { SOLVED } \\
\text { (MG/L } \\
\text { AS MG) }\end{array}$ & $\begin{array}{l}\text { SODIUM, } \\
\text { DIS- } \\
\text { SOLVED } \\
\text { (MG/L } \\
\text { AS NA) }\end{array}$ & $\begin{array}{l}\text { SODIUM } \\
\text { AD- } \\
\text { SORP- } \\
\text { TION } \\
\text { RATIO }\end{array}$ & $\begin{array}{l}\text { POTAS- } \\
\text { SIUM, } \\
\text { DIS- } \\
\text { SOLVED } \\
\text { (MG / L } \\
\text { AS K) }\end{array}$ & $\begin{array}{c}\text { BICAR- } \\
\text { BONATE } \\
\text { (MC/L } \\
\text { AS } \\
\text { HCO3) }\end{array}$ & $\begin{array}{l}\text { SULFATE } \\
\text { DIS- } \\
\text { SOLVED } \\
\text { (MG/L } \\
\text { AS S04) }\end{array}$ & $\begin{array}{l}\text { CHLO- } \\
\text { RIDE, } \\
\text { DIS- } \\
\text { SOLVED } \\
\text { (MG/L } \\
\text { AS CL) }\end{array}$ \\
\hline
\end{tabular}

\begin{tabular}{|c|c|c|c|c|c|c|c|c|c|}
\hline $\mathrm{FEB}$ & & & & & & & & & \\
\hline $03 \ldots$ & 58 & 31 & 7.3 & 25 & 1.1 & 5.5 & 60 & 54 & 42 \\
\hline $\begin{array}{l}03 \ldots \\
03 \ldots\end{array}$ & $-\overline{59}$ & $31^{--}$ & $7 . \overline{4}$ & $24^{--}$ & $1 . \overline{0}$ & $5 . \overline{5}$ & 60 & $54^{--}$ & 41 \\
\hline
\end{tabular}

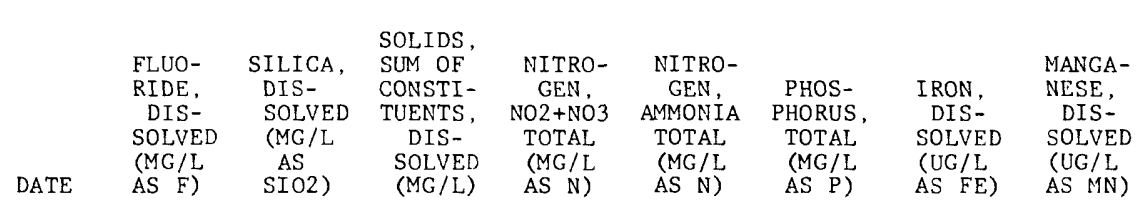

\begin{tabular}{|c|c|c|c|c|c|c|c|c|}
\hline \multicolumn{9}{|l|}{ FEB } \\
\hline $03 \ldots$ & .2 & 10 & 205 & .01 & .020 & .050 & 10 & 0 \\
\hline $03 \ldots$ & -- & & -- & .00 & .000 & .050 & 60 & 50 \\
\hline $03 \ldots$ & .3 & 10 & 203 & .01 & .020 & .050 & 0 & 20 \\
\hline
\end{tabular}

301754096380801 SOMERVILLE LAKE SITE FC

\begin{tabular}{|c|c|c|c|c|c|c|c|c|c|}
\hline DATE & TIME & $\begin{array}{l}\text { SAMP - } \\
\text { LING } \\
\text { DEPTH } \\
\text { (FT) }\end{array}$ & $\begin{array}{l}\text { SPE- } \\
\text { CIFIC } \\
\text { CON- } \\
\text { DUCT- } \\
\text { ANCE } \\
\text { (MICRO- } \\
\text { MHOS) }\end{array}$ & $\begin{array}{c}\text { PH } \\
\text { FIELD } \\
\text { (UNITS) }\end{array}$ & $\begin{array}{c}\text { TEMPER- } \\
\text { ATURE, } \\
\text { WATER } \\
\left(\begin{array}{lll}\text { DEG }\end{array}\right)\end{array}$ & $\begin{array}{l}\text { TRANS- } \\
\text { PAR- } \\
\text { ENCY } \\
\text { (SECCHI } \\
\text { DISK) } \\
(M)\end{array}$ & $\begin{array}{c}\text { OXYGEN, } \\
\text { DIS- } \\
\text { SOLVED } \\
\text { (MG/L) }\end{array}$ & $\begin{array}{c}\text { OXYGEN, } \\
\text { DIS- } \\
\text { SOLVED } \\
\text { (PER- } \\
\text { CENT } \\
\text { SATUR- } \\
\text { ATION) }\end{array}$ & $\begin{array}{l}\text { HARD- } \\
\text { NESS } \\
\text { (MG/L } \\
\text { AS } \\
\text { CACO3) }\end{array}$ \\
\hline $\begin{array}{l}\text { FEB } \\
03 \ldots \\
03 \ldots\end{array}$ & $\begin{array}{l}1630 \\
1632\end{array}$ & $14^{1.0}$ & $\begin{array}{l}545 \\
536\end{array}$ & $\begin{array}{l}7.7 \\
7.6\end{array}$ & $\begin{array}{l}12.5 \\
12.5\end{array}$ & .43 & $\begin{array}{l}10.3 \\
10.2\end{array}$ & $\begin{array}{l}96 \\
95\end{array}$ & $\begin{array}{l}160 \\
150\end{array}$ \\
\hline DATE & $\begin{array}{l}\text { HARD- } \\
\text { NESS, } \\
\text { NONCAR- } \\
\text { BONATE } \\
\text { (MG/L } \\
\text { CACO3) }\end{array}$ & $\begin{array}{l}\text { CALCIUM } \\
\text { DIS- } \\
\text { SOLVED } \\
\text { (MG/L } \\
\text { AS CA) }\end{array}$ & $\begin{array}{l}\text { MAGNE- } \\
\text { SIUM, } \\
\text { DIS- } \\
\text { SOLVED } \\
\text { (MG/L } \\
\text { AS MC) }\end{array}$ & $\begin{array}{l}\text { SODIUM, } \\
\text { DIS- } \\
\text { SOLVED } \\
\text { (MG/L } \\
\text { AS NA) }\end{array}$ & $\begin{array}{c}\text { SODIUM } \\
\text { AD- } \\
\text { SORP- } \\
\text { TION } \\
\text { RATIO }\end{array}$ & $\begin{array}{l}\text { POTAS- } \\
\text { SIUM, } \\
\text { DIS- } \\
\text { SOLVED } \\
\text { (MG/L } \\
\text { AS K) }\end{array}$ & $\begin{array}{c}\text { BICAR- } \\
\text { BONATE } \\
\text { (MG/L } \\
\text { AS } \\
\text { HCO3) }\end{array}$ & $\begin{array}{l}\text { SULFATE } \\
\text { DIS- } \\
\text { SOLVED } \\
\text { (MG/L } \\
\text { AS S04) }\end{array}$ & $\begin{array}{l}\text { CHLO- } \\
\text { RIDE, } \\
\text { DIS- } \\
\text { SOLVED } \\
\text { (MG/L } \\
\text { AS CL) }\end{array}$ \\
\hline
\end{tabular}

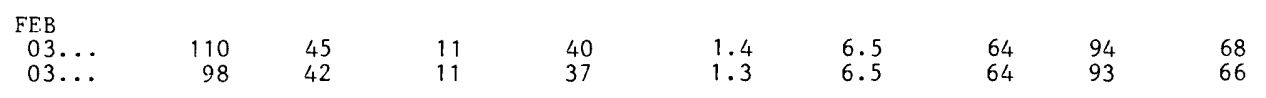

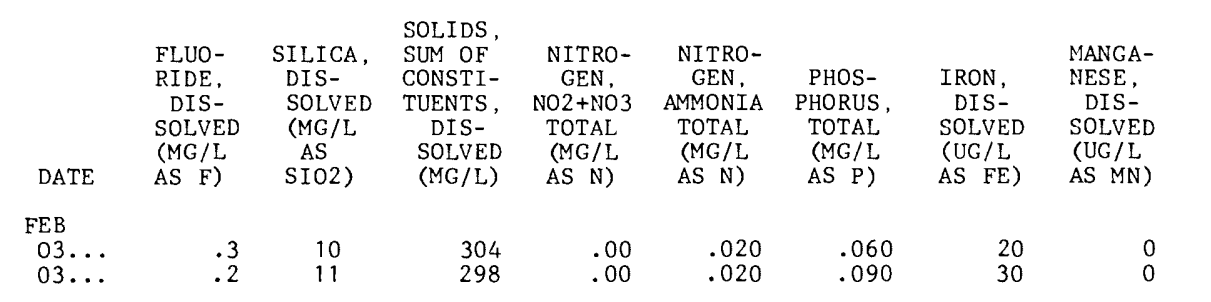


$\mathrm{FT}=$ feet $;$ MICROMHOS = micromhos per centimeter at $25^{\circ}$ Celsius; ${ }^{\circ} \mathrm{C}$ - degrees Celsius; $\mathrm{MC} / \mathrm{L}=\mathrm{milligrams}$ per liter; $\mathrm{UG} / \mathrm{L}=$ micrograms per liter

301908096313101 SOMERVILLE LAKE SITE AC

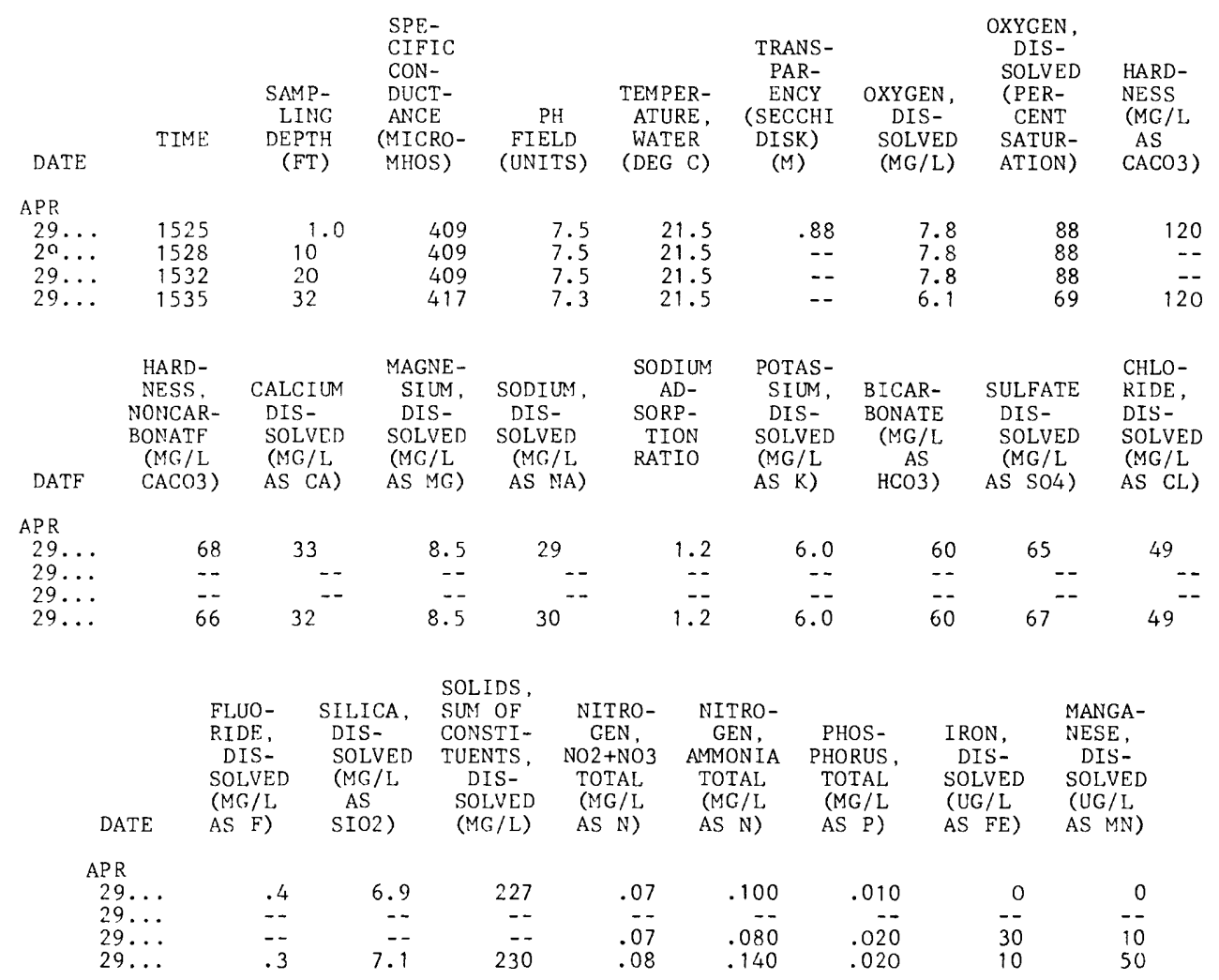

301940096315801 SOMERVILLE LAKE SITE AL

\begin{tabular}{|c|c|c|c|c|c|c|c|}
\hline DATE & TIME & $\begin{array}{l}\text { SAMP- } \\
\text { LING } \\
\text { DEPTH } \\
(\text { FT })\end{array}$ & $\begin{array}{l}\text { SPE- } \\
\text { CIFIC } \\
\text { CON- } \\
\text { DUCT- } \\
\text { ANCE } \\
\text { (MICRO- } \\
\text { MHOS) }\end{array}$ & $\begin{array}{c}\text { PH } \\
\text { FIELD } \\
\text { (UNITS) }\end{array}$ & $\begin{array}{l}\text { TEMPER- } \\
\text { ATURE, } \\
\text { WATER } \\
\text { (DEG C) }\end{array}$ & $\begin{array}{c}\text { OXYGEN, } \\
\text { DIS- } \\
\text { SOLVED } \\
\text { (MG/L) }\end{array}$ & $\begin{array}{c}\text { OXYGEN, } \\
\text { DIS- } \\
\text { SOLVED } \\
\text { (PER- } \\
\text { CENT } \\
\text { SATUR- } \\
\text { ATION) }\end{array}$ \\
\hline \multicolumn{8}{|l|}{ APR } \\
\hline $\begin{array}{l}29 \ldots \\
29 \ldots \\
29 \ldots\end{array}$ & $\begin{array}{l}1520 \\
1522 \\
1523\end{array}$ & $\begin{array}{l}1.0 \\
10^{\circ} \\
18\end{array}$ & $\begin{array}{l}409 \\
409 \\
409\end{array}$ & $\begin{array}{l}7.7 \\
7.7 \\
7.8\end{array}$ & $\begin{array}{l}22.0 \\
22.0 \\
21.5\end{array}$ & $\begin{array}{l}8.6 \\
8.6 \\
9.0\end{array}$ & $\begin{array}{r}98 \\
98 \\
101\end{array}$ \\
\hline
\end{tabular}

302026096341501 SOMERVILLE LAKE SITE BC

\begin{tabular}{|c|c|c|c|c|c|c|c|}
\hline DATE & TIME & $\begin{array}{c}\text { SAMP- } \\
\text { LINC } \\
\text { DEPTH } \\
\text { (FT) }\end{array}$ & $\begin{array}{l}\text { SPE- } \\
\text { CIFIC } \\
\text { CON- } \\
\text { DUCT- } \\
\text { ANCE } \\
\text { (MICRO- } \\
\text { MHOS) }\end{array}$ & $\begin{array}{c}\text { PH } \\
\text { FIELD } \\
\text { (UNITS) }\end{array}$ & $\begin{array}{c}\text { TEMPER- } \\
\text { ATURE, } \\
\text { WATER } \\
\text { (DEG C) }\end{array}$ & $\begin{array}{l}\text { TRANS- } \\
\text { PAR- } \\
\text { ENCY } \\
\text { (SECCHI } \\
\text { DISK) } \\
\text { (M) }\end{array}$ & $\begin{array}{c}\text { OXYGEN, } \\
\text { DIS- } \\
\text { SOLVED } \\
\text { (MG/L) }\end{array}$ \\
\hline $\begin{array}{l}\mathrm{APR} \\
29 \ldots \\
29 \ldots \\
29 \ldots\end{array}$ & $\begin{array}{l}1500 \\
1505 \\
1507\end{array}$ & $\begin{array}{l}1.0 \\
10^{1.0} \\
15\end{array}$ & $\begin{array}{l}404 \\
404 \\
404\end{array}$ & $\begin{array}{l}7.7 \\
7.7 \\
7.7\end{array}$ & $\begin{array}{l}22.5 \\
22.5 \\
22.0\end{array}$ & $\begin{array}{c}.67 \\
-- \\
--\end{array}$ & $\begin{array}{l}8.3 \\
8.3 \\
8.4\end{array}$ \\
\hline
\end{tabular}

$-43-$ 
Table 4--CHEMICAL-QUALITY SURVEYS OF SOMERVILLE LAKE APRIL 29, 1976--Continued

302026096341501 SOMERVILLE LAKE SITE BC--Continued

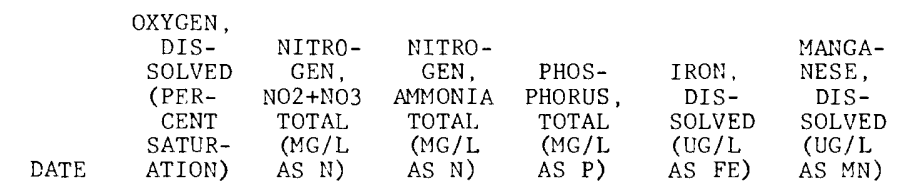

\begin{tabular}{|c|c|c|c|c|c|c|}
\hline $\begin{array}{l}\text { APR } \\
29 \ldots\end{array}$ & 94 & .04 & .080 & .010 & 30 & 0 \\
\hline $29 \ldots$ & 94 & .- & -. & -. & -- & - - \\
\hline $29 \ldots$ & 95 & .04 & .060 & .020 & 0 & 0 \\
\hline
\end{tabular}

301805096332501 SOMERVILLE LAKE SITE CC

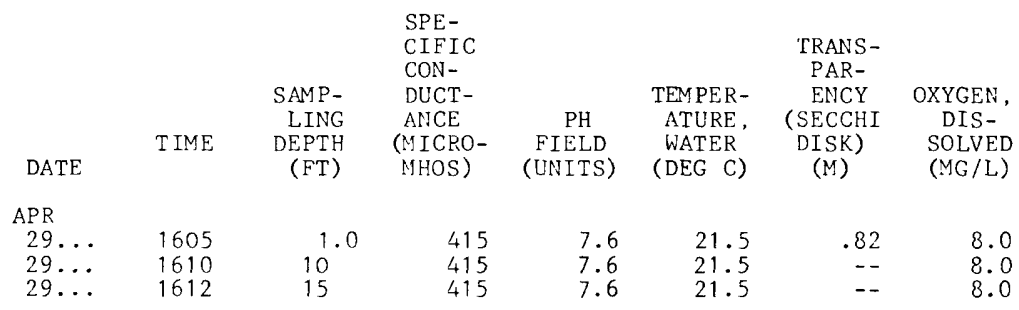

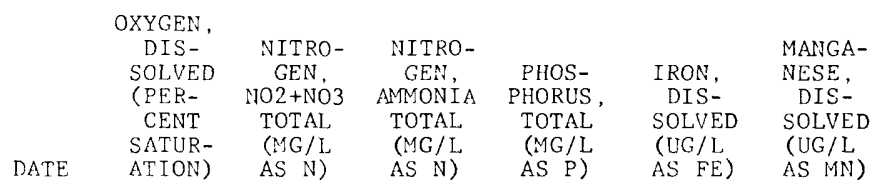

\begin{tabular}{|c|c|c|c|c|c|c|}
\hline \multicolumn{7}{|l|}{$A P R$} \\
\hline $29 \ldots$ & 90 & .08 & .080 & .020 & 20 & 0 \\
\hline $29 \ldots$ & 90 & -- & $\cdots$ & $\cdots$ & -- & -- \\
\hline $29 \ldots$ & 90 & .08 & .080 & .010 & 0 & 0 \\
\hline
\end{tabular}

301847096334601 SOMERVILLE LAKE SITE DR

\begin{tabular}{|c|c|c|c|c|c|c|c|}
\hline DATE & TIME & $\begin{array}{r}\text { SAMP- } \\
\text { LING } \\
\text { DEPTH } \\
(\mathrm{FT})\end{array}$ & $\begin{array}{l}\text { SPE- } \\
\text { CIFIC } \\
\text { CON- } \\
\text { DUCI- } \\
\text { ANCE } \\
\text { (MICRO- } \\
\text { MHOS) }\end{array}$ & $\begin{array}{c}\text { PH } \\
\text { FIELD } \\
\text { (UNI'TS) }\end{array}$ & $\begin{array}{c}\text { TEMPER- } \\
\text { ATURE, } \\
\text { WATER } \\
\text { (DEG C) }\end{array}$ & $\begin{array}{c}\text { OXYGEN, } \\
\text { DIS- } \\
\text { SOLVED } \\
\text { (MG/L) }\end{array}$ & $\begin{array}{l}\text { OXYGEN, } \\
\text { DIS- } \\
\text { SOLVED } \\
\text { (PER- } \\
\text { CENT } \\
\text { SATUR- } \\
\text { ATION) }\end{array}$ \\
\hline $\begin{array}{l}A P R \\
29 \ldots \\
29 \ldots \\
29 \ldots\end{array}$ & $\begin{array}{l}1630 \\
1633 \\
1635\end{array}$ & $\begin{array}{l}1.0 \\
10^{1.0} \\
24\end{array}$ & $\begin{array}{l}419 \\
419 \\
419\end{array}$ & $\begin{array}{l}7.7 \\
7.7 \\
7.7\end{array}$ & $\begin{array}{l}22.0 \\
22.0 \\
21.5\end{array}$ & $\begin{array}{l}8.1 \\
8.1 \\
8.6\end{array}$ & $\begin{array}{l}92 \\
92 \\
97\end{array}$ \\
\hline
\end{tabular}

301904096335601 SOMERVILLE LAKE SITE DC

\begin{tabular}{|c|c|c|c|c|c|c|c|c|c|}
\hline DATE & TIME & $\begin{array}{l}\text { SAMP- } \\
\text { LING } \\
\text { DEPTH } \\
(F T)\end{array}$ & $\begin{array}{l}\text { SPE- } \\
\text { CIFIC } \\
\text { CON- } \\
\text { DUCT- } \\
\text { ANCE } \\
\text { (MICRO- } \\
\text { MHOS) }\end{array}$ & $\begin{array}{c}\text { PH } \\
\text { FIELD } \\
\text { (UNITS) }\end{array}$ & $\begin{array}{l}\text { TEMPER- } \\
\text { ATURE, } \\
\text { WATER } \\
\left(\begin{array}{ll}\text { DEG } & \text { C) }\end{array}\right.\end{array}$ & $\begin{array}{l}\text { TRANS- } \\
\text { PAR- } \\
\text { ENCY } \\
\text { (SECCHI } \\
\text { DISK) } \\
\text { (M) }\end{array}$ & $\begin{array}{c}\text { OXYGEN, } \\
\text { DIS - } \\
\text { SOLVED } \\
\text { (MG/L) }\end{array}$ & $\begin{array}{c}\text { OXYGEN, } \\
\text { DIS- } \\
\text { SOLVED } \\
\text { (PER- } \\
\text { CENT } \\
\text { SATUR- } \\
\text { ATION) }\end{array}$ & $\begin{array}{l}\text { HARD- } \\
\text { NESS } \\
\text { (MG/L } \\
\text { AS } \\
\text { CACO3) }\end{array}$ \\
\hline $\begin{array}{l}\text { APR } \\
29 \ldots \\
29 \ldots \\
29 \ldots \\
29 \ldots\end{array}$ & $\begin{array}{l}1640 \\
1643 \\
1646 \\
1649\end{array}$ & $\begin{array}{l}1.0 \\
10 \\
20 \\
27\end{array}$ & $\begin{array}{l}419 \\
419 \\
419 \\
419\end{array}$ & $\begin{array}{l}7.7 \\
7.7 \\
7.7 \\
7.7\end{array}$ & $\begin{array}{l}22.0 \\
22.0 \\
22.0 \\
22.0\end{array}$ & $\begin{array}{l}.73 \\
-- \\
--\end{array}$ & $\begin{array}{l}8.0 \\
8.0 \\
8.0 \\
8.0\end{array}$ & $\begin{array}{l}91 \\
91 \\
91 \\
91\end{array}$ & $\begin{array}{r}120 \\
-- \\
120\end{array}$ \\
\hline
\end{tabular}


Table 4--CHEMICAL-QUALITY SURVEYS OF SOMERVILLE LAKE APRIL 29, 1976--Continued

301904096335601 SOMERVILLE LAKE DC--Continued

\begin{tabular}{|c|c|c|c|c|c|c|c|c|c|}
\hline ATE & $\begin{array}{c}\text { HARD- } \\
\text { NESS, } \\
\text { NONCAR- } \\
\text { BONATE } \\
\text { (MG/L } \\
\text { CACO3) }\end{array}$ & $\begin{array}{l}\text { CALCIUM } \\
\text { DIS- } \\
\text { SOLVED } \\
\text { (MG/L } \\
\text { AS CA) }\end{array}$ & $\begin{array}{l}\text { MAGNE- } \\
\text { SIUM, } \\
\text { DIS- } \\
\text { SOLVED } \\
\text { (MG/L } \\
\text { AS MG) }\end{array}$ & $\begin{array}{l}\text { SODIUM, } \\
\text { DIS- } \\
\text { SOLVED } \\
\text { (MG/L } \\
\text { AS NA) }\end{array}$ & $\begin{array}{l}\text { SODIUM } \\
\text { AD- } \\
\text { SORP- } \\
\text { TION } \\
\text { RATIO }\end{array}$ & $\begin{array}{l}\text { POTAS- } \\
\text { SIUM, } \\
\text { DIS- } \\
\text { SOLVED } \\
\text { (MG/L } \\
\text { AS K) }\end{array}$ & $\begin{array}{c}\text { BICAR- } \\
\text { BONATE } \\
\text { (MG/L } \\
\text { AS } \\
\mathrm{HCO} 3 \text { ) }\end{array}$ & $\begin{array}{l}\text { SULFATE } \\
\text { DIS- } \\
\text { SOLVED } \\
\text { (MG/L } \\
\text { AS S04) }\end{array}$ & $\begin{array}{l}\text { CHLO- } \\
\text { RIDE, } \\
\text { DIS- } \\
\text { SOLVED } \\
\text { (MG/L } \\
\text { AS CL) }\end{array}$ \\
\hline
\end{tabular}

$29 \ldots$

$29 \ldots$

$29 .$.

$\begin{array}{lr}67 & 32 \\ -- & --\end{array}$

8.730

1.26 .0

$6.0 \quad 60$

$67 \quad 50$

$8 . \overline{1} 29^{--}$

$\overline{1.2}$

$6 . \overline{0}$

60

$69^{--}$

50

$50^{--}$

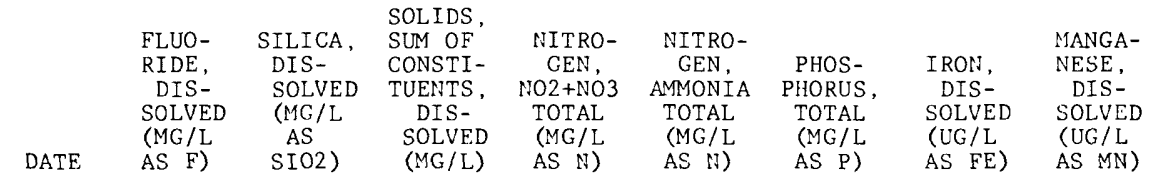

\begin{tabular}{|c|c|c|c|c|c|c|c|c|}
\hline $\begin{array}{l}\text { APR } \\
29 \ldots\end{array}$ & .3 & 7.1 & 231 & .06 & .090 & .020 & 10 & 10 \\
\hline $29 \ldots$ & -- & -- & -- & -- & -- & -- & -- & -- \\
\hline $29 \ldots$ & - & -- & - & .06 & .070 & .020 & 20 & 0 \\
\hline $29 \ldots$ & .2 & 7.1 & 232 & .06 & .080 & .020 & 30 & 0 \\
\hline
\end{tabular}

301817096364101 SOMERVILLE LAKE SITE EC

\begin{tabular}{|c|c|c|c|c|c|c|c|c|c|}
\hline DATE. & T IME & $\begin{array}{r}\text { SAMP- } \\
\text { LING } \\
\text { DEPTH } \\
\text { (FT) }\end{array}$ & $\begin{array}{l}\text { SPE- } \\
\text { CIFIC } \\
\text { CON- } \\
\text { DUCT- } \\
\text { ANCE } \\
\text { (MICRO- } \\
\text { MHOS) }\end{array}$ & $\begin{array}{c}\text { PH } \\
\text { FIELD } \\
\text { (UNITS) }\end{array}$ & $\begin{array}{l}\text { TEMPER- } \\
\text { ATURE, } \\
\text { WATER } \\
\text { (DEG C) }\end{array}$ & $\begin{array}{l}\text { TRANS- } \\
\text { PAR- } \\
\text { ENCY } \\
\text { (SECCHI } \\
\text { DISK) } \\
\text { (M) }\end{array}$ & $\begin{array}{c}\text { OXYGEN } \\
\text { DIS- } \\
\text { SOLVED } \\
(M G / L)\end{array}$ & $\begin{array}{c}\text { OXYGEN, } \\
\text { DIS- } \\
\text { SOLVED } \\
\text { (PER- } \\
\text { CENT } \\
\text { SATUR- } \\
\text { ATIOII) }\end{array}$ & $\begin{array}{l}\text { HARD- } \\
\text { NESS } \\
\text { (MG / L } \\
\text { AS } \\
\text { CACO3) }\end{array}$ \\
\hline $\begin{array}{c}\mathrm{APR} \\
29 \ldots \\
29 \ldots \\
29 \ldots\end{array}$ & $\begin{array}{l}1715 \\
1717 \\
1720\end{array}$ & $\begin{array}{l}10^{1.0} \\
21\end{array}$ & $\begin{array}{l}395 \\
390 \\
386\end{array}$ & $\begin{array}{l}7.4 \\
7.3 \\
7.3\end{array}$ & $\begin{array}{l}22.0 \\
22.0 \\
22.0\end{array}$ & $\begin{array}{r}.52 \\
-- \\
--\end{array}$ & $\begin{array}{l}7.2 \\
7.2 \\
7.0\end{array}$ & $\begin{array}{l}82 \\
82 \\
80\end{array}$ & $\begin{array}{r}110 \\
-- \\
100\end{array}$ \\
\hline ATE & $\begin{array}{l}\text { HARD- } \\
\text { NESS } \\
\text { NONCAR- } \\
\text { BONATE } \\
\text { (MG /L } \\
\text { CACO3) }\end{array}$ & $\begin{array}{l}\text { CALCIUM } \\
\text { DIS- } \\
\text { SOLVED } \\
\text { (MG/L } \\
\text { AS CA) }\end{array}$ & $\begin{array}{l}\text { MAGNE- } \\
\text { SIUM, } \\
\text { DIS- } \\
\text { SOLVED } \\
\text { (MG/L } \\
\text { AS MG) }\end{array}$ & $\begin{array}{l}\text { SODIUM, } \\
\text { DIS- } \\
\text { SOLVED } \\
\text { (MG/L } \\
\text { AS NA) }\end{array}$ & $\begin{array}{c}\text { SODIUM } \\
\text { AD- } \\
\text { SORP- } \\
\text { TION } \\
\text { PATIO }\end{array}$ & $\begin{array}{l}\text { POTAS- } \\
\text { SIUM, } \\
\text { DIS- } \\
\text { SOLVED } \\
(M G / L \\
\text { AS K) }\end{array}$ & $\begin{array}{c}\text { BICAR- } \\
\text { BONATE } \\
\text { (MG/L } \\
\text { AS } \\
\text { HCO3) }\end{array}$ & $\begin{array}{l}\text { SULFATE } \\
\text { DIS- } \\
\text { SOLVED } \\
\text { (MG/L } \\
\text { AS SO4) }\end{array}$ & $\begin{array}{l}\text { CHLO- } \\
\text { RIDE } \\
\text { DIS- } \\
\text { SOLVED } \\
\text { (MG/L } \\
\text { AS CL) }\end{array}$ \\
\hline
\end{tabular}

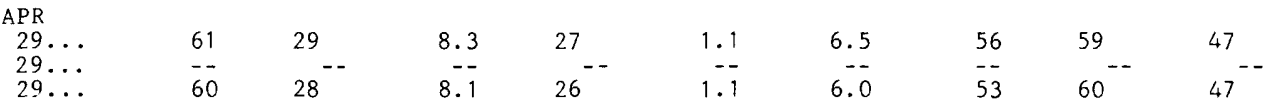

\begin{tabular}{|c|c|c|c|c|c|c|c|c|}
\hline & $\begin{array}{l}\text { FLUO- } \\
\text { RIDE, } \\
\text { DIS- } \\
\text { SOLVED } \\
\text { (MG/L }\end{array}$ & $\begin{array}{l}\text { SILICA, } \\
\text { DIS- } \\
\text { SOLVED } \\
\text { (MG/L } \\
\text { AS }\end{array}$ & $\begin{array}{l}\text { SOLIDS, } \\
\text { SUM OF } \\
\text { CONSTI- } \\
\text { TUENTS, } \\
\text { DIS- } \\
\text { SOLVED }\end{array}$ & $\begin{array}{l}\text { NITRO- } \\
\text { GEN } \\
\text { NO2+NO3 } \\
\text { TOTAL } \\
\text { (MG/L }\end{array}$ & $\begin{array}{l}\text { NITRO- } \\
\text { GEN, } \\
\text { AMMONIA } \\
\text { TOTAL } \\
\text { (MG/L }\end{array}$ & $\begin{array}{l}\text { PHOS- } \\
\text { PHORUS } \\
\text { TOTAL } \\
\text { (MG/L }\end{array}$ & $\begin{array}{l}\text { IRON, } \\
\text { DIS- } \\
\text { SOLVED } \\
\text { (UG/L }\end{array}$ & $\begin{array}{l}\text { MANGA- } \\
\text { NESE, } \\
\text { DIS- } \\
\text { SOLVED } \\
\text { (UG/L }\end{array}$ \\
\hline DATE & & & & & & & & \\
\hline
\end{tabular}

\begin{tabular}{|c|c|c|c|c|c|c|c|c|}
\hline APR & & & & & & & & \\
\hline $29 \ldots$ & $\begin{array}{l}.4 \\
--\end{array}$ & 10 & $\begin{array}{r}215 \\
--\end{array}$ & $\begin{array}{l}.10 \\
.09\end{array}$ & $\begin{array}{l}.110 \\
.170\end{array}$ & $\begin{array}{l}.050 \\
.050\end{array}$ & $\begin{array}{l}80 \\
90\end{array}$ & $\begin{array}{r}0 \\
10\end{array}$ \\
\hline $29 \ldots$ & .3 & 10 & 212 & .09 & .110 & .050 & 80 & 0 \\
\hline
\end{tabular}


Table 4--CHEMICAL-QUALITY SURVEYS OF SOMERVILLE LAKE APRIL 29, 1976--Continued

301754096380801 SOMERVILLE LAKE SITE FC

\begin{tabular}{|c|c|c|c|c|c|c|c|c|c|}
\hline DATE & TIME & $\begin{array}{l}\text { SAMP- } \\
\text { LING } \\
\text { DEPTH } \\
(\mathrm{FT})\end{array}$ & $\begin{array}{l}\text { SPE- } \\
\text { CIFIC } \\
\text { CON- } \\
\text { DUCT- } \\
\text { ANCE } \\
\text { (MICRO- } \\
\text { MHOS) }\end{array}$ & $\begin{array}{c}\text { PH } \\
\text { FIELD } \\
\text { (UNITS) }\end{array}$ & $\begin{array}{c}\text { TEMPER- } \\
\text { ATURE, } \\
\text { WATER } \\
\text { (DEG C) }\end{array}$ & $\begin{array}{l}\text { TRANS- } \\
\text { PAR- } \\
\text { ENCY } \\
\text { (SECCHI } \\
\text { DISK) } \\
\text { (M) }\end{array}$ & $\begin{array}{l}\text { OXYGEN, } \\
\text { DIS- } \\
\text { SOLVED } \\
\text { (MG/L) }\end{array}$ & $\begin{array}{c}\text { OXYGEN, } \\
\text { DIS- } \\
\text { SOLVED } \\
\text { (PER- } \\
\text { CENT } \\
\text { SATUR- } \\
\text { ATION) }\end{array}$ & $\begin{array}{l}\text { HARD- } \\
\text { NESS } \\
(\mathrm{MG} / \mathrm{L} \\
\text { AS } \\
\text { CACO3) }\end{array}$ \\
\hline $\begin{array}{l}A P R \\
29 \ldots \\
29 \ldots \\
29 \ldots\end{array}$ & $\begin{array}{l}1740 \\
1745 \\
1747\end{array}$ & $\begin{array}{l}1.0 \\
10^{17}\end{array}$ & $\begin{array}{l}343 \\
346 \\
346\end{array}$ & $\begin{array}{l}7.2 \\
7.2 \\
7.2\end{array}$ & $\begin{array}{l}22.0 \\
22.0 \\
22.0\end{array}$ & $\begin{array}{l}.27 \\
-- \\
--\end{array}$ & $\begin{array}{l}6.8 \\
6.8 \\
7.2\end{array}$ & $\begin{array}{l}77 \\
77 \\
82\end{array}$ & $\begin{array}{l}93 \\
-1 \\
94\end{array}$ \\
\hline DATE & $\begin{array}{l}\text { HARD- } \\
\text { NESS } \\
\text { NONCAR- } \\
\text { BONATE } \\
\text { (MG/L } \\
\text { CACO3) }\end{array}$ & $\begin{array}{l}\text { CALCIUM } \\
\text { DIS- } \\
\text { SOLVED } \\
\text { (MG/L } \\
\text { AS CA) }\end{array}$ & $\begin{array}{l}\text { MAGNE- } \\
\text { SIUM, } \\
\text { DIS- } \\
\text { SOLVED } \\
\text { (MG/L } \\
\text { AS MG) }\end{array}$ & $\begin{array}{l}\text { SODIUM, } \\
\text { DIS- } \\
\text { SOLVED } \\
\text { (MG/L } \\
\text { AS NA) }\end{array}$ & $\begin{array}{c}\text { SODIUM } \\
\text { AD- } \\
\text { SORP- } \\
\text { TION } \\
\text { RATIO }\end{array}$ & $\begin{array}{l}\text { POTAS- } \\
\text { SIUM, } \\
\text { DIS- } \\
\text { SOLVED } \\
\text { (MG/L } \\
\text { AS K) }\end{array}$ & $\begin{array}{c}\text { BICAR- } \\
\text { BONATE } \\
\text { (MG /L } \\
\text { AS } \\
\text { HCO3) }\end{array}$ & $\begin{array}{l}\text { SULFATE } \\
\text { DIS- } \\
\text { SOLVED } \\
\text { (MG/L } \\
\text { AS S04) }\end{array}$ & $\begin{array}{l}\text { CHLO- } \\
\text { RIDE, } \\
\text { DIS- } \\
\text { SOLVED } \\
\text { (MG/L } \\
\text { AS CL) }\end{array}$ \\
\hline
\end{tabular}

\begin{tabular}{|c|c|c|c|c|c|c|c|c|c|}
\hline APR & & & & & & & & & \\
\hline $29 \ldots$ & 50 & 25 & 7.3 & 24 & 1.1 & 6.5 & 52 & 51 & 42 \\
\hline $\begin{array}{l}29 \ldots \\
29 \ldots\end{array}$ & 51 & $25^{--}$ & $7 . \overline{7}$ & $24^{--}$ & $1 . \overline{1}$ & $6 . \overline{5}$ & $\overline{52}$ & $53^{--}$ & 42 \\
\hline
\end{tabular}

\begin{tabular}{|c|c|c|c|c|c|c|c|c|}
\hline DATE & $\begin{array}{l}\text { FLUUO- } \\
\text { RIDF, } \\
\text { DIS- } \\
\text { SOLVED } \\
\text { (MG/L } \\
\text { AS F) }\end{array}$ & $\begin{array}{l}\text { SILICA, } \\
\text { DIS- } \\
\text { SOLVED } \\
\text { (MG/L } \\
\text { AS } \\
\text { SIO2) }\end{array}$ & $\begin{array}{l}\text { SOLIDS, } \\
\text { SUM OF } \\
\text { CONSTI- } \\
\text { TUENTS, } \\
\text { DIS- } \\
\text { SOLVED } \\
\text { (MG/L) }\end{array}$ & $\begin{array}{c}\text { NITRO- } \\
\text { GEN, } \\
\text { NO2+NO3 } \\
\text { TOTAL } \\
\text { (MG/I } \\
\text { AS N) }\end{array}$ & $\begin{array}{l}\text { NITRO- } \\
\text { GEN, } \\
\text { AMMONIA } \\
\text { TOTAL } \\
\text { (MG/L } \\
\text { AS N) }\end{array}$ & $\begin{array}{c}\text { PHOS- } \\
\text { PHORUS, } \\
\text { TOTAL } \\
\text { (MG/L } \\
\text { AS P) }\end{array}$ & $\begin{array}{l}\text { IRON, } \\
\text { DIS- } \\
\text { SOLVED } \\
\text { (UG/L } \\
\text { AS FE) }\end{array}$ & $\begin{array}{l}\text { MANGA- } \\
\text { NESE, } \\
\text { DIS- } \\
\text { SOLVED } \\
\text { (UG / L } \\
\text { AS MN) }\end{array}$ \\
\hline \multicolumn{9}{|l|}{ APR } \\
\hline $29 \ldots$ & .4 & 12 & 194 & .05 & .130 & .000 & 90 & 10 \\
\hline $29 \ldots$ & -- & -- & -- & .05 & .140 & .070 & 130 & 0 \\
\hline $29 \ldots$ & .3 & 11 & 195 & .06 & .150 & .090 & 90 & 0 \\
\hline
\end{tabular}




\begin{tabular}{|c|c|c|c|c|c|c|c|c|c|}
\hline & & & 90809631 & 3101 SOM & ERVILLE I & AKE SITE & $A C$ & & \\
\hline DATE & TIME & $\begin{array}{l}\text { SAMP- } \\
\text { LING } \\
\text { DEPTH } \\
\text { (FT) }\end{array}$ & $\begin{array}{l}\text { SPE- } \\
\text { CIFIC } \\
\text { CON- } \\
\text { DUCT- } \\
\text { ANCE } \\
\text { (MICRO- } \\
\text { MHOS) }\end{array}$ & $\begin{array}{c}\text { PH } \\
\text { FIELD } \\
\text { (UNITS) }\end{array}$ & $\begin{array}{l}\text { TEMPER- } \\
\text { ATURE, } \\
\text { WATER } \\
\left(\begin{array}{ll}\text { DEG } & \text { C) }\end{array}\right.\end{array}$ & $\begin{array}{l}\text { TRANS- } \\
\text { PAR- } \\
\text { ENCY } \\
\text { (SECCHI } \\
\text { DISK) } \\
\text { (M) }\end{array}$ & $\begin{array}{c}\text { OXYCEN, } \\
\text { DIS- } \\
\text { SOLVED } \\
(\text { MG } / L)\end{array}$ & $\begin{array}{l}\text { OXYGEN, } \\
\text { DIS- } \\
\text { SOLVED } \\
\text { (PER- } \\
\text { CENT } \\
\text { SATUR- } \\
\text { ATION) }\end{array}$ & $\begin{array}{l}\text { HARD- } \\
\text { NESS } \\
\text { (MG/L } \\
\text { AS } \\
\text { CACO3) }\end{array}$ \\
\hline $\begin{array}{l}\text { AUG } \\
20 \ldots \\
20 \ldots \\
20 \ldots \\
20 \ldots\end{array}$ & $\begin{array}{l}0900 \\
0904 \\
0908 \\
0910\end{array}$ & $\begin{array}{l}1.0 \\
10 \\
20 \\
27\end{array}$ & $\begin{array}{l}375 \\
375 \\
375 \\
375\end{array}$ & $\begin{array}{l}7.3 \\
7.3 \\
7.2 \\
7.2\end{array}$ & $\begin{array}{l}28.0 \\
28.0 \\
28.0 \\
28.0\end{array}$ & $\begin{array}{l}.58 \\
-- \\
-- \\
--\end{array}$ & $\begin{array}{l}4.8 \\
4.6 \\
4.4 \\
4.4\end{array}$ & $\begin{array}{l}62 \\
59 \\
56 \\
56\end{array}$ & $\begin{array}{r}98 \\
-- \\
100\end{array}$ \\
\hline DATE & $\begin{array}{c}\text { HARD- } \\
\text { NESS, } \\
\text { NONCAR- } \\
\text { BONATE } \\
\text { (MG/L } \\
\text { CACO3) }\end{array}$ & $\begin{array}{l}\text { CALCIUM } \\
\text { DIS- } \\
\text { SOLVED } \\
\text { (MG/L } \\
\text { AS CA) }\end{array}$ & $\begin{array}{l}\text { MAGNE- } \\
\text { SIUM, } \\
\text { DIS- } \\
\text { SOLVED } \\
\text { (MG/L } \\
\text { AS MG) }\end{array}$ & $\begin{array}{l}\text { SODIUM, } \\
\text { DIS- } \\
\text { SOLVED } \\
\text { (MG/L } \\
\text { AS NA) }\end{array}$ & $\begin{array}{l}\text { SODIUM } \\
\text { AD- } \\
\text { SORP- } \\
\text { TION } \\
\text { RATIO }\end{array}$ & $\begin{array}{l}\text { POTAS- } \\
\text { SIUM, } \\
\text { DIS- } \\
\text { SOLVED } \\
\text { (MG/L } \\
\text { AS K) }\end{array}$ & $\begin{array}{c}\text { BICAR- } \\
\text { BONATE } \\
\text { (MG/L } \\
\text { AS } \\
\text { HCO3) }\end{array}$ & $\begin{array}{l}\text { SULFATE } \\
\text { DIS- } \\
\text { SOLVED } \\
\text { (MG/L } \\
\text { AS SO4) }\end{array}$ & $\begin{array}{l}\text { CHLO- } \\
\text { RIDE, } \\
\text { DIS- } \\
\text { SOLVED } \\
\text { (MG/L } \\
\text { AS CL) }\end{array}$ \\
\hline
\end{tabular}

\begin{tabular}{|c|c|c|c|c|c|c|c|c|c|}
\hline AUG & & & & & & & & & \\
\hline $20 \ldots$ & 46 & 27 & 7.4 & 26 & 1.1 & 5.8 & 63 & 52 & 44 \\
\hline $\begin{array}{l}20 \ldots \ldots \\
20 \ldots\end{array}$ & $=-$ & -- & $=-$ & -- & -- & -- & $=$ & $=-$ & \\
\hline & 52 & 29 & 7.3 & 26 & 1.1 & 5.8 & 62 & 51 & 44 \\
\hline
\end{tabular}

\begin{tabular}{|c|c|c|c|c|c|c|c|c|}
\hline DATE & $\begin{array}{l}\text { FLUO- } \\
\text { RIDE, } \\
\text { DIS- } \\
\text { SOLVED } \\
\text { (MG/L } \\
\text { AS F) }\end{array}$ & $\begin{array}{l}\text { SILICA, } \\
\text { DIS- } \\
\text { SOLVED } \\
\text { (MG/L } \\
\text { AS } \\
\text { SIO2) }\end{array}$ & $\begin{array}{c}\text { SOLIDS, } \\
\text { SUM OF } \\
\text { CONSTI- } \\
\text { TUENTS, } \\
\text { DIS- } \\
\text { SOLVED } \\
(\text { MG/L) }\end{array}$ & $\begin{array}{c}\text { MI'TRO- } \\
\text { GEN, } \\
\text { NO2+NO3 } \\
\text { TOTAL } \\
\text { (MG/L } \\
\text { AS N) }\end{array}$ & $\begin{array}{l}\text { NITRO- } \\
\text { CEN, } \\
\text { AMMONIA } \\
\text { TOTAL } \\
\text { (MG/L } \\
\text { AS N) }\end{array}$ & $\begin{array}{l}\text { PHOS- } \\
\text { PHORUS, } \\
\text { TOTAL } \\
\text { (MG/L } \\
\text { AS P) }\end{array}$ & $\begin{array}{l}\text { IRON, } \\
\text { DIS- } \\
\text { SOLVED } \\
\text { (UC/L } \\
\text { AS FE) }\end{array}$ & $\begin{array}{l}\text { MANGA- } \\
\text { NESE, } \\
\text { DIS- } \\
\text { SOLVED } \\
\text { (UG/L } \\
\text { AS MN) }\end{array}$ \\
\hline \multicolumn{9}{|l|}{ AUC } \\
\hline $20 \ldots$ & .2 & 12 & 205 & .01 & .010 & .020 & 10 & 10 \\
\hline $20 \ldots$ & -. & -- & - & .0 & .08 &. & -- & 20 \\
\hline $20 \ldots$ & .3 & 12 & 206 & .01 & .030 & .030 & 20 & 30 \\
\hline
\end{tabular}

301940096315801 SOMERVILLE LAKE SITE AL

\begin{tabular}{|c|c|c|c|c|c|c|c|}
\hline DATE & TIME & $\begin{array}{c}\text { SAMP- } \\
\text { LING } \\
\text { DEPTH } \\
(\text { FT })\end{array}$ & $\begin{array}{l}\text { SPE- } \\
\text { CIFIC } \\
\text { CON- } \\
\text { DUCT- } \\
\text { ANCE } \\
\text { (MICRO- } \\
\text { MHOS) }\end{array}$ & $\begin{array}{c}\text { PH } \\
\text { FIELD } \\
\text { (UNITS) }\end{array}$ & $\begin{array}{l}\text { TEMPER- } \\
\text { ATURE, } \\
\text { WATER } \\
\text { (DEG C) }\end{array}$ & $\begin{array}{c}\text { OXYGEN, } \\
\text { DIS- } \\
\text { SOLVED } \\
\text { (MG/L) }\end{array}$ & $\begin{array}{c}\text { OXYGEN, } \\
\text { DIS- } \\
\text { SOLVED } \\
\text { (PER- } \\
\text { CENT } \\
\text { SATUR- } \\
\text { ATION) }\end{array}$ \\
\hline $\begin{array}{l}\text { AUG } \\
20 \ldots \\
20 \ldots \\
20 \ldots \\
20 \ldots\end{array}$ & $\begin{array}{l}0920 \\
0924 \\
0928 \\
0930\end{array}$ & $\begin{array}{l}1.0 \\
10 \\
20 \\
26\end{array}$ & $\begin{array}{l}375 \\
375 \\
375 \\
375\end{array}$ & $\begin{array}{l}7.5 \\
7.4 \\
7.4 \\
7.4\end{array}$ & $\begin{array}{l}28.5 \\
28.5 \\
28.5 \\
28.0\end{array}$ & $\begin{array}{l}5.2 \\
4.9 \\
4.8 \\
4.8\end{array}$ & $\begin{array}{l}68 \\
64 \\
62 \\
62\end{array}$ \\
\hline
\end{tabular}

302026096341501 SOMERVILLE LAKE SITE BC

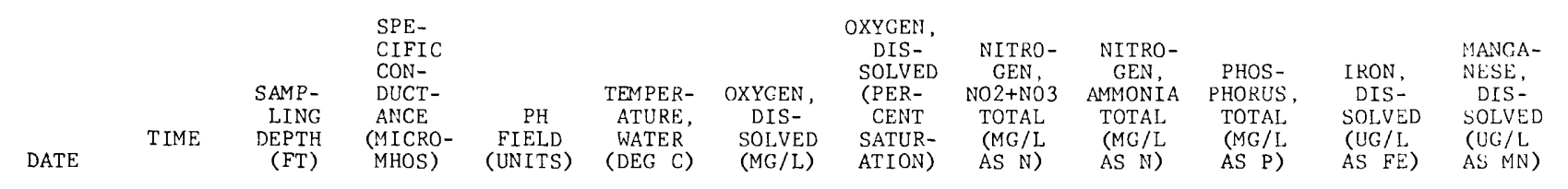

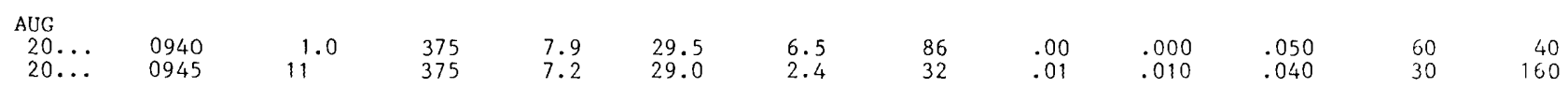


Table 5--CHEMICAL-QUALITY SURVEYS OF SOMERVILLE LAKE AUGUST 20, 1976--Continued

301805096332501 SOMERVILLE LAKE SITE CC

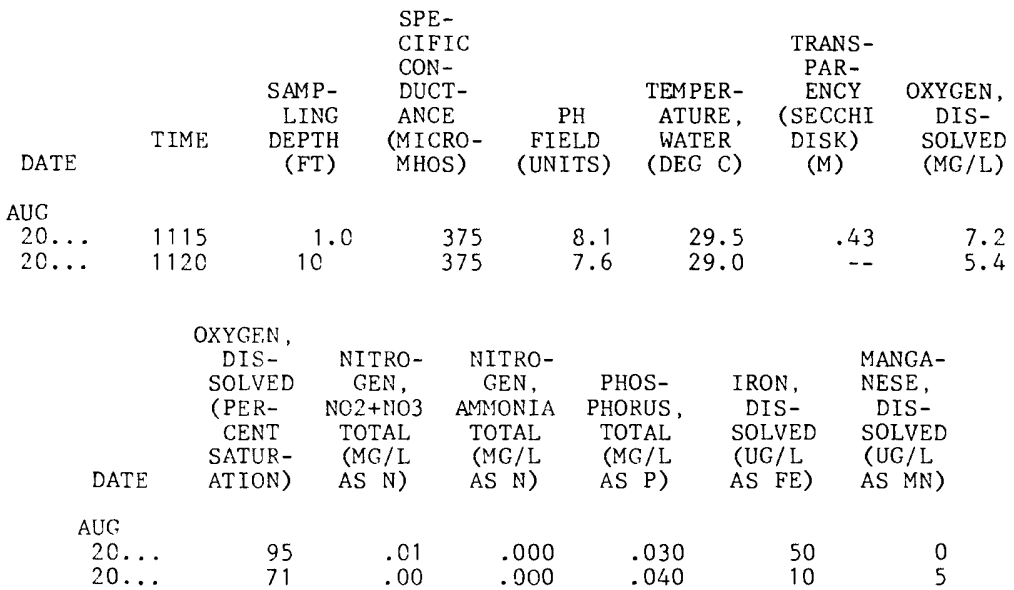

301817096364101 SOMERVILLE LAKE SITE EC

\begin{tabular}{|c|c|c|c|c|c|c|c|}
\hline DATE & TIME & $\begin{array}{l}\text { SAMP- } \\
\text { LING } \\
\text { DEPTH } \\
(\mathrm{FT})\end{array}$ & $\begin{array}{l}\text { SPE- } \\
\text { CIFIC } \\
\text { CON- } \\
\text { DUCT- } \\
\text { ANCE } \\
\text { (MICRO- } \\
\text { MHOS) }\end{array}$ & $\begin{array}{c}\text { PH } \\
\text { FIELD } \\
\text { (UNITS) }\end{array}$ & 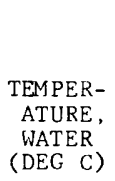 & $\begin{array}{c}\text { OXYGEN, } \\
\text { DIS- } \\
\text { SOLVED } \\
\text { (MG/L) }\end{array}$ & $\begin{array}{c}\text { OXYGEN, } \\
\text { DIS- } \\
\text { SOLVED } \\
\text { (PER- } \\
\text { CENT } \\
\text { SATUR- } \\
\text { ATION) }\end{array}$ \\
\hline AUG & & & & & & & \\
\hline $\begin{array}{l}20 \ldots \\
20 \ldots \\
20 \ldots\end{array}$ & $\begin{array}{l}1017 \\
1020 \\
1025\end{array}$ & $\begin{array}{l}1.0 \\
10 \\
21\end{array}$ & $\begin{array}{l}380 \\
380 \\
380\end{array}$ & $\begin{array}{l}8.2 \\
7.9 \\
7.5\end{array}$ & $\begin{array}{l}29.0 \\
29.0 \\
29.0\end{array}$ & $\begin{array}{l}7.4 \\
6.6 \\
5.4\end{array}$ & $\begin{array}{l}97 \\
87 \\
71\end{array}$ \\
\hline
\end{tabular}

301847096334601 SOMERVILLE LAKE SITE DR

\begin{tabular}{|c|c|c|c|c|c|c|c|}
\hline DATE & TIME & $\begin{array}{l}\text { SAMP- } \\
\text { LING } \\
\text { DEPTH } \\
\text { (FT) }\end{array}$ & $\begin{array}{l}\text { SPE- } \\
\text { CIFIC } \\
\text { CON- } \\
\text { DUCT- } \\
\text { ANCE } \\
\text { (MICRO- } \\
\text { MHOS) }\end{array}$ & $\begin{array}{c}\text { PH } \\
\text { FIELD } \\
\text { (UNITS) }\end{array}$ & $\begin{array}{c}\text { TEMPER- } \\
\text { ATURE, } \\
\text { WATER } \\
\text { (DEG C) }\end{array}$ & $\begin{array}{c}\text { OXYGEN, } \\
\text { DIS- } \\
\text { SOLVED } \\
\text { (MG/L) }\end{array}$ & $\begin{array}{l}\text { OXYGEN, } \\
\text { DIS- } \\
\text { SOLVED } \\
\text { (PER- } \\
\text { CENT } \\
\text { SATUR- } \\
\text { ATION) }\end{array}$ \\
\hline AUC & & & & & & & \\
\hline $\begin{array}{l}20 \ldots \\
20 \ldots \\
20 \ldots\end{array}$ & $\begin{array}{l}1010 \\
1012 \\
1014\end{array}$ & $\begin{array}{l}1.0 \\
10 \\
21\end{array}$ & $\begin{array}{l}378 \\
378 \\
378\end{array}$ & $\begin{array}{l}7.9 \\
7.6 \\
7.5\end{array}$ & $\begin{array}{l}29.0 \\
28.5 \\
28.5\end{array}$ & $\begin{array}{l}6.6 \\
5.8 \\
4.4\end{array}$ & $\begin{array}{l}87 \\
75 \\
57\end{array}$ \\
\hline
\end{tabular}

301904096335601 SOMERVILLE LAKE SITE DC

\begin{tabular}{|c|c|c|c|c|c|c|c|c|c|}
\hline DATE & TIME & $\begin{array}{l}\text { SAMP- } \\
\text { LING } \\
\text { DEPTH } \\
\text { (FT) }\end{array}$ & $\begin{array}{l}\text { SPE- } \\
\text { CIFIC } \\
\text { CON- } \\
\text { DUCT- } \\
\text { ANCE } \\
\text { (MICRO- } \\
\text { MHOS) }\end{array}$ & $\begin{array}{c}\text { PH } \\
\text { FIELD } \\
\text { (UNITS) }\end{array}$ & $\begin{array}{c}\text { TEMPER- } \\
\text { ATURE, } \\
\text { WATER } \\
\left(\begin{array}{ll}\text { DEG } & \text { C) }\end{array}\right.\end{array}$ & $\begin{array}{l}\text { TRANS- } \\
\text { PAR- } \\
\text { ENCY } \\
\text { (SECCHI } \\
\text { DISK) } \\
\text { (M) }\end{array}$ & $\begin{array}{c}\text { OXYGEN, } \\
\text { DIS- } \\
\text { SOLVED } \\
\text { (MG/L) }\end{array}$ & $\begin{array}{l}\text { OXYGEN, } \\
\text { DIS- } \\
\text { SOLVED } \\
\text { (PER- } \\
\text { CENT } \\
\text { SATUR- } \\
\text { ATION) }\end{array}$ & $\begin{array}{l}\text { HARD- } \\
\text { NESS } \\
\text { (MG/L } \\
\text { AS } \\
\text { CACO3) }\end{array}$ \\
\hline \multicolumn{10}{|l|}{ AUG } \\
\hline $\begin{array}{l}20 \ldots \\
20 \ldots \\
20 \ldots\end{array}$ & $\begin{array}{l}1000 \\
1002 \\
1004\end{array}$ & $\begin{array}{l}1.0 \\
10^{\circ} \\
22\end{array}$ & $\begin{array}{l}378 \\
378 \\
378\end{array}$ & $\begin{array}{l}7.7 \\
7.5 \\
7.4\end{array}$ & $\begin{array}{l}29.0 \\
28.5 \\
28.5\end{array}$ & $\begin{array}{c}.52 \\
-- \\
--\end{array}$ & $\begin{array}{l}6.2 \\
5.4 \\
4.7\end{array}$ & $\begin{array}{l}82 \\
70 \\
61\end{array}$ & $\begin{array}{r}100 \\
100\end{array}$ \\
\hline
\end{tabular}


Table 5--CHEMICAL-QUALITY SURVEYS OF SOMERVILLE LAKE AUGUST 20, 1976--Continued

301904096335601 SOMERVILLE LAKE SITE DC--Continued

\begin{tabular}{|c|c|c|c|c|c|c|c|c|c|}
\hline $\mathrm{TI}$ & $\begin{array}{c}\text { HARD- } \\
\text { NESS } \\
\text { NONCAR- } \\
\text { BONATE } \\
\text { (MG/L } \\
\text { CACO3) }\end{array}$ & $\begin{array}{l}\text { CALCI UM } \\
\text { DIS- } \\
\text { SOLVED } \\
\text { (MG/L } \\
\text { AS CA) }\end{array}$ & $\begin{array}{l}\text { MAGNE- } \\
\text { SIUM, } \\
\text { DIS- } \\
\text { SOLVED } \\
\text { (MG/L } \\
\text { AS MG) }\end{array}$ & $\begin{array}{l}\text { SODIUM, } \\
\text { DIS- } \\
\text { SOLVED } \\
\text { (MG/L } \\
\text { AS NA) }\end{array}$ & $\begin{array}{c}\text { SODIUM } \\
\text { AD- } \\
\text { SORP- } \\
\text { TION } \\
\text { RATIO }\end{array}$ & $\begin{array}{l}\text { POTAS- } \\
\text { SIUM, } \\
\text { DIS- } \\
\text { SOLVED } \\
\text { (MG/L } \\
\text { AS K) }\end{array}$ & $\begin{array}{c}\text { BICAR- } \\
\text { BONATE } \\
\text { (MG / L } \\
\text { AS } \\
\text { HCO3) }\end{array}$ & $\begin{array}{l}\text { SULFATE } \\
\text { DIS- } \\
\text { SOLVED } \\
\text { (MG/L } \\
\text { AS SO4) }\end{array}$ & $\begin{array}{l}\text { CHLO- } \\
\text { RIDE, } \\
\text { DIS- } \\
\text { SOLVED } \\
(1 \mathrm{G} / \mathrm{L} \\
\text { AS CL) }\end{array}$ \\
\hline
\end{tabular}

\begin{tabular}{|c|c|c|c|c|c|c|c|c|c|}
\hline \multicolumn{10}{|l|}{ AUG } \\
\hline $20 \ldots$ & 48 & 28 & 7.5 & 26 & 1.1 & 5.8 & 64 & 52 & 43 \\
\hline $20 \ldots$ & -- & -- & $\ldots$ & - & -- & -- & $\ldots$ & & 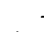 \\
\hline $20 \ldots$ & 48 & 28 & 7.4 & 26 & 1.1 & 5.8 & 64 & 51 & 44 \\
\hline
\end{tabular}

\begin{tabular}{|c|c|c|c|c|c|c|c|c|}
\hline & $\begin{array}{l}\text { FLUO- } \\
\text { RIDE, } \\
\text { DIS- } \\
\text { SOLVED } \\
\text { (MG/L }\end{array}$ & $\begin{array}{l}\text { SILICA, } \\
\text { DIS- } \\
\text { SOLVED } \\
\text { (MC/L } \\
\text { AS }\end{array}$ & $\begin{array}{l}\text { SOLIDS, } \\
\text { SUM OF } \\
\text { CONSTI- } \\
\text { TUENTS, } \\
\text { DIS- } \\
\text { SOLVED }\end{array}$ & $\begin{array}{l}\text { NITRO- } \\
\text { GEN, } \\
\text { NO2+NO3 } \\
\text { TOTAL } \\
\text { (MG/L }\end{array}$ & $\begin{array}{l}\text { NITRO- } \\
\text { GEN, } \\
\text { AMMONIA } \\
\text { TOTAL } \\
\text { (MG / L }\end{array}$ & $\begin{array}{l}\text { PHOS- } \\
\text { PHORUS, } \\
\text { TOTAL } \\
(\mathrm{MG} / \mathrm{L}\end{array}$ & $\begin{array}{l}\text { IRON, } \\
\text { DIS- } \\
\text { SOLVED } \\
\text { (UG/L }\end{array}$ & $\begin{array}{l}\text { MANGA- } \\
\text { NESE, } \\
\text { DIS- } \\
\text { SOLVED } \\
\text { (UG/L }\end{array}$ \\
\hline TE & AS F) & SIO2) & $(M G / L)$ & AS $N$ ) & AS $N$ ) & $A S \quad P)$ & AS FE) & AS MN) \\
\hline
\end{tabular}

\begin{tabular}{|c|c|c|c|c|c|c|c|c|}
\hline \multicolumn{9}{|l|}{ AUG } \\
\hline $20 \ldots$ & .2 & 12 & 206 & .01 & .010 & .020 & 30 & 5 \\
\hline $20 \ldots$ & -- & & -- & -- & -- & -- & -- & -- \\
\hline $20 \ldots$ & .2 & 12 & 206 & .01 & .000 & .030 & 20 & 90 \\
\hline
\end{tabular}

301754096380801 SOMERVILLE LAKE SITE FC

\begin{tabular}{|c|c|c|c|c|c|c|c|c|c|}
\hline DATE & T IME & $\begin{array}{l}\text { SAMP- } \\
\text { LING } \\
\text { DEPTH } \\
(\text { FT })\end{array}$ & $\begin{array}{l}\text { SPE- } \\
\text { CIFIC } \\
\text { CON- } \\
\text { DUCI- } \\
\text { ANCE } \\
\text { (MICRO- } \\
\text { MHOS) }\end{array}$ & $\begin{array}{c}\text { PH } \\
\text { FIELD } \\
\text { (UNITS) }\end{array}$ & $\begin{array}{l}\text { TEMPER- } \\
\text { ATURE } \\
\text { WATER } \\
\text { (DEG } \\
\text { (D) }\end{array}$ & $\begin{array}{c}\text { TRANS- } \\
\text { PAR- } \\
\text { ENCY } \\
\text { (SECCHI } \\
\text { DISK) } \\
(M)\end{array}$ & $\begin{array}{c}\text { OXYGEN, } \\
\text { DIS- } \\
\text { SOLVED } \\
(M G / L)\end{array}$ & $\begin{array}{c}\text { OXYGEN, } \\
\text { DIS- } \\
\text { SOLVED } \\
\text { (PER- } \\
\text { CENT } \\
\text { SATUR- } \\
\text { ATION) }\end{array}$ & $\begin{array}{l}\text { HARD- } \\
\text { NESS } \\
(\mathrm{MG} / \mathrm{L} \\
\text { AS } \\
\mathrm{CACO3)}\end{array}$ \\
\hline $\begin{array}{l}\text { AUG } \\
20 \ldots \\
20 \ldots\end{array}$ & $\begin{array}{l}1035 \\
1040\end{array}$ & $13^{1.0}$ & $\begin{array}{l}408 \\
418\end{array}$ & $\begin{array}{l}8.3 \\
7.6\end{array}$ & $\begin{array}{l}29.0 \\
28.5\end{array}$ & .34 & $\begin{array}{l}7.3 \\
5.4\end{array}$ & $\begin{array}{l}96 \\
70\end{array}$ & $\begin{array}{l}110 \\
110\end{array}$ \\
\hline DATE & $\begin{array}{l}\text { HARD- } \\
\text { NESS, } \\
\text { NONCAR- } \\
\text { BONATE } \\
\text { (MG/L } \\
\text { CACO3) }\end{array}$ & $\begin{array}{l}\text { CALCIUM } \\
\text { DIS- } \\
\text { SOLVED } \\
\text { (MG/L } \\
\text { AS CA) }\end{array}$ & $\begin{array}{l}\text { MAGNE- } \\
\text { SIUM, } \\
\text { DIS- } \\
\text { SOLVED } \\
\text { (MG/L } \\
\text { AS MG) }\end{array}$ & $\begin{array}{l}\text { SODIUM, } \\
\text { DIS- } \\
\text { SOLVED } \\
\text { (MG/L } \\
\text { AS NA) }\end{array}$ & $\begin{array}{c}\text { SODIUM } \\
\text { AD- } \\
\text { SORP- } \\
\text { TION } \\
\text { RATIO }\end{array}$ & $\begin{array}{l}\text { POTAS- } \\
\text { SIUM, } \\
\text { DIS- } \\
\text { SOLVED } \\
(M G / L \\
\text { AS K) }\end{array}$ & $\begin{array}{c}\text { BICAR- } \\
\text { BONATE } \\
\text { (MG/L } \\
\text { AS } \\
\text { HCO3) }\end{array}$ & $\begin{array}{l}\text { SULFATE } \\
\text { DIS- } \\
\text { SOLVED } \\
\text { (MC/L } \\
\text { AS SO4) }\end{array}$ & $\begin{array}{l}\text { CHLO- } \\
\text { RIDE, } \\
\text { DIS- } \\
\text { SOLVED } \\
\text { (MG/L } \\
\text { AS CL) }\end{array}$ \\
\hline
\end{tabular}

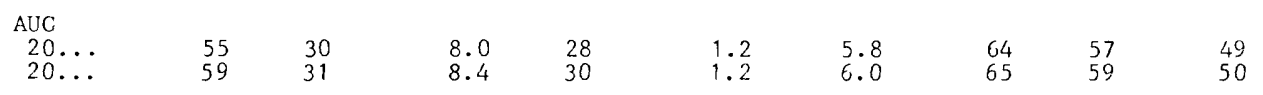

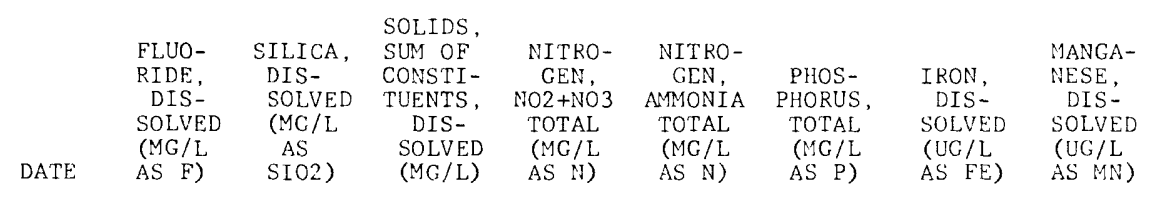

\begin{tabular}{|c|c|c|c|c|c|c|c|c|}
\hline AUG & & & & & & & & \\
\hline $20 \ldots$ & .3 & 13 & 223 & .01 & .010 & .060 & 20 & 5 \\
\hline $20 \ldots$ & .3 & 13 & 230 & .01 & .020 & .070 & 30 & 50 \\
\hline
\end{tabular}


Table 6--CHEMICAL-QUALITY SURVEYS OF SOMERVILLE LAKE FEBRUARY 16, 1977

\begin{tabular}{|c|c|c|c|c|c|c|c|c|c|}
\hline & & & 190809631 & 3101 & ERVILLE & AKE SITE & $\mathrm{AC}$ & & \\
\hline DATE & TIME & $\begin{array}{l}\text { SAMP- } \\
\text { LINC } \\
\text { DEPTH } \\
\text { (FT) }\end{array}$ & $\begin{array}{l}\text { SPE- } \\
\text { CIFIC } \\
\text { CON- } \\
\text { DUCT- } \\
\text { ANCE } \\
\text { (MICRO- } \\
\text { MHOS) }\end{array}$ & $\begin{array}{c}\text { PH } \\
\text { FIELD } \\
\text { (UNITS) }\end{array}$ & $\begin{array}{c}\text { TEMPER- } \\
\text { ATURE, } \\
\text { WATER } \\
\left(\begin{array}{ll}\text { DEG } & \text { C) }\end{array}\right.\end{array}$ & $\begin{array}{l}\text { TRANS- } \\
\text { PAR- } \\
\text { ENCY } \\
\text { (SECCHI } \\
\text { DISK) } \\
\text { (M) }\end{array}$ & $\begin{array}{c}\text { OXYGEN, } \\
\text { DIS- } \\
\text { SOLVED } \\
\text { (MG /L) }\end{array}$ & $\begin{array}{c}\text { OXYGEN, } \\
\text { DIS- } \\
\text { SOLVED } \\
\text { (PER- } \\
\text { CENT } \\
\text { SATUR- } \\
\text { ATION) }\end{array}$ & $\begin{array}{l}\text { HARD- } \\
\text { NESS } \\
\text { (MG/L } \\
\text { AS } \\
\text { CACO3) }\end{array}$ \\
\hline $\begin{array}{l}\text { EB } \\
16 \ldots \\
16 \ldots \\
16 \ldots \\
16 \ldots\end{array}$ & $\begin{array}{l}1535 \\
1537 \\
1540 \\
1545\end{array}$ & $\begin{array}{l}1.0 \\
10 \\
20 \\
32\end{array}$ & $\begin{array}{l}336 \\
335 \\
335 \\
336\end{array}$ & $\begin{array}{l}7.7 \\
7.7 \\
7.7 \\
7.6\end{array}$ & $\begin{array}{l}12.0 \\
12.0 \\
12.0 \\
12.0\end{array}$ & $\begin{array}{c}.43 \\
-- \\
-- \\
--\end{array}$ & $\begin{array}{l}12.0 \\
12.0 \\
12.0 \\
12.0\end{array}$ & $\begin{array}{l}115 \\
115 \\
115 \\
115\end{array}$ & $\begin{array}{l}93 \\
-- \\
92\end{array}$ \\
\hline NATE & $\begin{array}{l}\text { HARD- } \\
\text { NESS } \\
\text { NONCAR- } \\
\text { BONATE } \\
\text { (MG/L } \\
\text { CACO3) }\end{array}$ & $\begin{array}{l}\text { CALCIUM } \\
\text { DIS- } \\
\text { SOLVED } \\
\text { (MG/L } \\
\text { AS CA) }\end{array}$ & $\begin{array}{l}\text { MAGNE- } \\
\text { SIUM, } \\
\text { DIS- } \\
\text { SOLVED } \\
\text { (MG/L } \\
\text { AS MG) }\end{array}$ & $\begin{array}{l}\text { SODIUM, } \\
\text { DIS- } \\
\text { SOLVED } \\
\text { (MG/L } \\
\text { AS NA) }\end{array}$ & $\begin{array}{c}\text { SODIUM } \\
\text { AD- } \\
\text { SORP- } \\
\text { TION } \\
\text { RATIO }\end{array}$ & $\begin{array}{l}\text { POTAS- } \\
\text { SIUM, } \\
\text { DIS- } \\
\text { SOLVED } \\
(M G / L \\
\text { AS K) }\end{array}$ & $\begin{array}{c}\text { BICAR- } \\
\text { BONATE } \\
\text { (MG /L } \\
\text { AS } \\
\text { HCO3) }\end{array}$ & $\begin{array}{l}\text { SULFATE } \\
\text { DIS- } \\
\text { SOLVED } \\
\text { (MG/L } \\
\text { AS SO4) }\end{array}$ & $\begin{array}{l}\text { CHLO- } \\
\text { RIDE, } \\
\text { DIS- } \\
\text { SOLVED } \\
\text { (MG/L } \\
\text { AS CL) }\end{array}$ \\
\hline
\end{tabular}

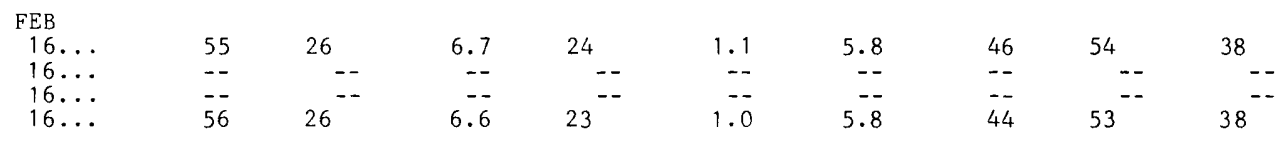

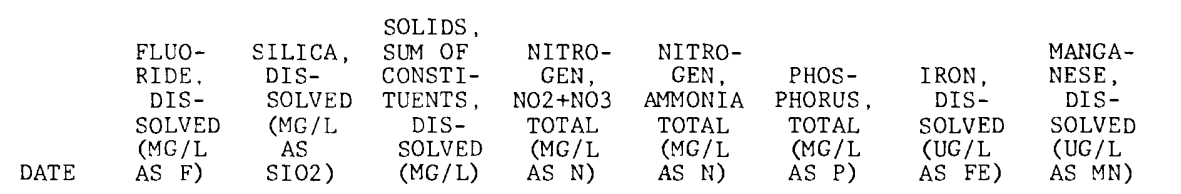

\begin{tabular}{|c|c|c|c|c|c|c|c|c|}
\hline FEB & & & & & & & & \\
\hline $16 \ldots$ & .1 & 10 & 187 & .13 & .080 & .050 & 40 & 0 \\
\hline $16 \ldots$ & -- & -- & -- & $\rightarrow$ & $\cdots$ & -- & -- & - \\
\hline $16 \ldots$ & -- & -- & -- & -- & -- & -- & -- & -- \\
\hline $16 \ldots$ & .1 & 10 & 184 & .13 & .050 & .060 & 100 & 60 \\
\hline
\end{tabular}

301940096315801 SOMERVILLE LAKE SITE AL

\begin{tabular}{|c|c|c|c|c|c|c|c|}
\hline DATE & T IMF & $\begin{array}{l}\text { SAMP- } \\
\text { LING } \\
\text { DEPTH } \\
\text { (ET) }\end{array}$ & $\begin{array}{l}\text { SPE- } \\
\text { CIFIC } \\
\text { CON- } \\
\text { DUCT- } \\
\text { ANCE } \\
\text { (MICRO- } \\
\text { MHOS) }\end{array}$ & $\begin{array}{c}\text { PH } \\
\text { FIELD } \\
\text { (UNITS) }\end{array}$ & $\begin{array}{l}\text { TEMPER- } \\
\text { ATURE, } \\
\text { WATER } \\
\left(\begin{array}{lll}\text { DEG }\end{array}\right)\end{array}$ & $\begin{array}{c}\text { OXYGEN, } \\
\text { DIS- } \\
\text { SOLVED } \\
(\mathrm{MG} / \mathrm{L})\end{array}$ & $\begin{array}{c}\text { OXYGEN, } \\
\text { DIS- } \\
\text { SOLVED } \\
\text { (PER- } \\
\text { CENT } \\
\text { SATUR- } \\
\text { ATION) }\end{array}$ \\
\hline \multicolumn{8}{|l|}{$\mathrm{FEB}$} \\
\hline $\begin{array}{l}16 \ldots \\
16 \ldots \\
16 \ldots \\
16 \ldots\end{array}$ & $\begin{array}{l}1355 \\
1357 \\
1359 \\
1402\end{array}$ & $\begin{array}{l}1.0 \\
10 \\
20 \\
29\end{array}$ & $\begin{array}{l}335 \\
335 \\
335 \\
335\end{array}$ & $\begin{array}{l}7.6 \\
7.6 \\
7.6 \\
7.5\end{array}$ & $\begin{array}{l}12.0 \\
12.0 \\
12.0 \\
12.0\end{array}$ & $\begin{array}{l}12.0 \\
11.9 \\
11.6 \\
11.6\end{array}$ & $\begin{array}{l}115 \\
114 \\
112 \\
112\end{array}$ \\
\hline
\end{tabular}

302026096341501 SOMERVILLE LAKE SITE BC

\begin{tabular}{|c|c|c|c|c|c|c|c|}
\hline DATE & TIME & $\begin{array}{l}\text { SAMP- } \\
\text { LING } \\
\text { DEPTH } \\
\text { (FT) }\end{array}$ & $\begin{array}{l}\text { SPE- } \\
\text { CIFIC } \\
\text { CON- } \\
\text { DUCT- } \\
\text { ANCE } \\
\text { (MICRO- } \\
\text { MHOS) }\end{array}$ & $\begin{array}{c}\text { PH } \\
\text { FIELD } \\
\text { (UNITS) }\end{array}$ & 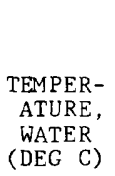 & $\begin{array}{l}\text { TRANS - } \\
\text { PAR- } \\
\text { ENCY } \\
\text { (SECCHI } \\
\text { DISK) } \\
(\mathrm{M})\end{array}$ & $\begin{array}{c}\text { OXYGEN, } \\
\text { DIS- } \\
\text { SOLVED } \\
\text { (MG/L) }\end{array}$ \\
\hline $\begin{array}{l}\mathrm{FEB} \\
16 \ldots \\
16 \ldots\end{array}$ & $\begin{array}{l}1610 \\
1615\end{array}$ & $15^{1.0}$ & $\begin{array}{l}295 \\
295\end{array}$ & $\begin{array}{l}7.4 \\
7.4\end{array}$ & $\begin{array}{l}11.0 \\
11.0\end{array}$ & .40 & $\begin{array}{l}11.2 \\
11.2\end{array}$ \\
\hline
\end{tabular}


302026096341501 SOMERVILLE LAKE SITE BC--Continued

\begin{tabular}{ccccccc}
\multicolumn{7}{c}{ OXYGEN, } \\
DIS- & NITRO- & NITRO- & & & \\
& SOLVED & GEN, & GEN, & PHOS- & IRON, & MANGA- \\
& (PER- & NO2+NO3 & AMMONIA & PHORUS, & DIS- & DIS- \\
& CENT & TOTAL & TOTAL & TOTAL & SOLVED & SOLVED \\
& SATUR- & (MG/L & (MG/L & (MG/L & (UG/L & (UG/L \\
DATE & ATION) & AS N) & AS N) & AS P) & AS FE) & AS MN)
\end{tabular}

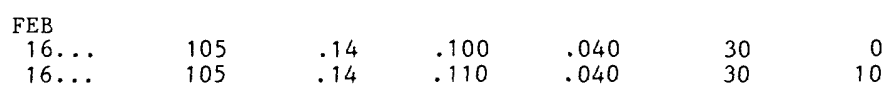

301805096332501 SOMERVILLE LAKE SITE CC

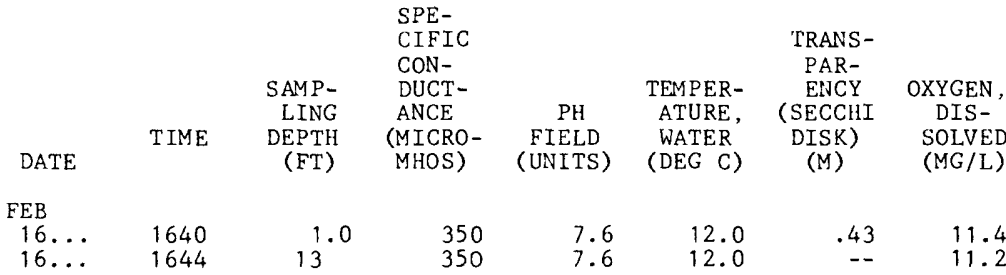

\begin{tabular}{ccccccc}
\multicolumn{7}{c}{ OXYGEN, } \\
DIS- & NITRO- & NITRO- & & & \\
& SOLVED & GEN, & GEN, & PHOS- & IRON, & NANGA- \\
& (PER- & NO2+NO3 & AMMONIA & PHORUS, & DIS- & DIS- \\
& CENT & TOTAL & TOTAL & TOTAL & SOLVED & SOLVED \\
& SATUR- & (MG/L & (MG/L & (MG/L & (UG/L & (UG/L \\
DATE & ATION) & AS N) & AS N) & AS P) & AS FE) & AS MN)
\end{tabular}

\begin{tabular}{|c|c|c|c|c|c|c|}
\hline FEB & & & & & & \\
\hline $\begin{array}{l}16 \ldots \\
16 \ldots\end{array}$ & $\begin{array}{l}110 \\
108\end{array}$ & $\begin{array}{r}.13 \\
.13\end{array}$ & $\begin{array}{r}.080 \\
.070\end{array}$ & $\begin{array}{l}.050 \\
.050\end{array}$ & $\begin{array}{l}40 \\
40\end{array}$ & $\begin{array}{r}10 \\
0\end{array}$ \\
\hline
\end{tabular}

301847096334601 SOMERVILLE LAKE SITE DR

\begin{tabular}{|c|c|c|c|c|c|c|c|}
\hline DATE & T IME & $\begin{array}{l}\text { SAMP- } \\
\text { LING } \\
\text { DEPTH } \\
\text { (FT) }\end{array}$ & $\begin{array}{l}\text { SPE- } \\
\text { CIFIC } \\
\text { CON- } \\
\text { DUCT- } \\
\text { ANCE } \\
\text { (MICRO- } \\
\text { MHOS) }\end{array}$ & $\begin{array}{c}\text { PH } \\
\text { FIELD } \\
\text { (UNITS) }\end{array}$ & 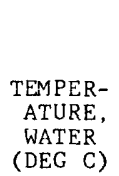 & $\begin{array}{c}\text { OXYGEN, } \\
\text { DIS- } \\
\text { SOLVED } \\
\text { (MG /L) }\end{array}$ & $\begin{array}{c}\text { OXYGEN, } \\
\text { DIS- } \\
\text { SOLVED } \\
\text { (PER- } \\
\text { CENT } \\
\text { SATUR- } \\
\text { ATION) }\end{array}$ \\
\hline \multicolumn{8}{|l|}{ FEB } \\
\hline $\begin{array}{l}16 \ldots \\
16 \ldots \\
16 \ldots \\
16 \ldots\end{array}$ & $\begin{array}{l}1650 \\
1652 \\
1655 \\
1658\end{array}$ & $\begin{array}{l}1.0 \\
10 \\
20 \\
28\end{array}$ & $\begin{array}{l}363 \\
363 \\
363 \\
320\end{array}$ & $\begin{array}{l}7.4 \\
7.4 \\
7.3 \\
7.3\end{array}$ & $\begin{array}{l}11.5 \\
11.5 \\
11.5 \\
10.5\end{array}$ & $\begin{array}{l}11.0 \\
11.0 \\
11.0 \\
10.9\end{array}$ & $\begin{array}{l}104 \\
104 \\
104 \\
101\end{array}$ \\
\hline
\end{tabular}

301904096335601 SOMERVILLE LAKE SITE DC

\begin{tabular}{|c|c|c|c|c|c|c|c|c|}
\hline DATE & TIME & $\begin{array}{c}\text { SAMP- } \\
\text { LING } \\
\text { DEPTH } \\
(\mathrm{FT})\end{array}$ & $\begin{array}{l}\text { SPE- } \\
\text { CIFIC } \\
\text { CON- } \\
\text { DUCT- } \\
\text { ANCE } \\
\text { (MICRO- } \\
\text { MHOS) }\end{array}$ & $\begin{array}{c}\text { PH } \\
\text { FIELD } \\
\text { (UNITS) }\end{array}$ & 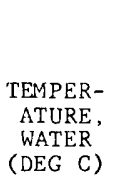 & $\begin{array}{l}\text { TRANS- } \\
\text { PAR- } \\
\text { ENCY } \\
\text { (SECCHI } \\
\text { DISK) } \\
\text { (M) }\end{array}$ & $\begin{array}{c}\text { OXYGEN, } \\
\text { DIS- } \\
\text { SOLVED } \\
(M G / L)\end{array}$ & $\begin{array}{c}\text { OXYGEN, } \\
\text { DIS- } \\
\text { SOLVED } \\
\text { (PER- } \\
\text { CENT } \\
\text { SATUR- } \\
\text { ATION) }\end{array}$ \\
\hline H & & & & & & & & \\
\hline $\begin{array}{l}16 \ldots \\
16 \ldots \\
16 \ldots \\
16 \ldots\end{array}$ & $\begin{array}{l}1625 \\
1627 \\
1630 \\
1633\end{array}$ & $\begin{array}{l}1.0 \\
10 \\
20 \\
28\end{array}$ & $\begin{array}{l}363 \\
345 \\
320 \\
320\end{array}$ & $\begin{array}{l}7.3 \\
7.3 \\
7.5 \\
7.5\end{array}$ & $\begin{array}{l}12.0 \\
11.0 \\
10.5 \\
10.5\end{array}$ & $\begin{array}{c}.49 \\
-- \\
-- \\
--\end{array}$ & $\begin{array}{l}10.8 \\
10.9 \\
11.1 \\
11.0\end{array}$ & $\begin{array}{l}104 \\
102 \\
103 \\
102\end{array}$ \\
\hline
\end{tabular}


Table 6--CHEMICAL-QUALITY SURVEYS OF SOMERVILLE LAKE FEBRUARY 16, 1977--Continued

301904096335601 SOMERVILLE LAKE SITE DC--Continued

\begin{tabular}{|c|c|c|c|c|c|c|c|c|c|}
\hline ATE & $\begin{array}{l}\text { HARD- } \\
\text { NESS } \\
(\mathrm{MG} / \mathrm{L} \\
\text { AS } \\
\text { CACO3) }\end{array}$ & $\begin{array}{l}\text { HARD- } \\
\text { NESS } \\
\text { NONCAR- } \\
\text { BONATE } \\
\text { (MG /L } \\
\text { CACO3) }\end{array}$ & $\begin{array}{l}\text { CALCIUM } \\
\text { DIS- } \\
\text { SOLVED } \\
\text { (MG/L } \\
\text { AS CA) }\end{array}$ & $\begin{array}{l}\text { MAGNE- } \\
\text { SIUM, } \\
\text { DIS- } \\
\text { SOLVED } \\
\text { (MG/L } \\
\text { AS MG) }\end{array}$ & $\begin{array}{l}\text { SODIUM, } \\
\text { DIS- } \\
\text { SOLVED } \\
\text { (MG/L } \\
\text { AS NA) }\end{array}$ & $\begin{array}{c}\text { SODIUM } \\
\text { AD- } \\
\text { SORP- } \\
\text { TION } \\
\text { RATIO }\end{array}$ & $\begin{array}{l}\text { POTAS- } \\
\text { SIUM, } \\
\text { DIS- } \\
\text { SOLVED } \\
(M G / L \\
\text { AS K) }\end{array}$ & $\begin{array}{c}\text { BICAR- } \\
\text { BONATE } \\
\text { (MG /L } \\
\text { AS } \\
\text { HCO3) }\end{array}$ & $\begin{array}{l}\text { SULFATE } \\
\text { DIS- } \\
\text { SOLVED } \\
\text { (MG/L } \\
\text { AS SO4) }\end{array}$ \\
\hline
\end{tabular}

\begin{tabular}{|c|c|c|c|c|c|c|c|c|}
\hline FEB & & & & & & & & \\
\hline $16 \ldots$ & 100 & 64 & 8 & 7.4 & 26 & 1.1 & 5.6 & 44 \\
\hline $16 \ldots$ & -- & -- & -- & -- & -- & -- & -- & -- \\
\hline $16 \ldots$ & - & -- & -- & - - & -- & -- & - & -- \\
\hline $16 \ldots$ & 86 & 49 & 4 & 6.4 & 23 & 1.1 & 6.2 & 46 \\
\hline DATE & $\begin{array}{l}\text { CHLO- } \\
\text { RIDE, } \\
\text { DIS- } \\
\text { SOLVED } \\
\text { (MG/L. } \\
\text { AS CI) }\end{array}$ & $\begin{array}{l}\text { SILICA, } \\
\text { DIS- } \\
\text { SOLVED } \\
\text { (MG/L } \\
\text { AS } \\
\text { SIO2) }\end{array}$ & $\begin{array}{c}\text { SOLIDS, } \\
\text { SUM OF } \\
\text { CONSTI- } \\
\text { TUENTS, } \\
\text { DIS- } \\
\text { SOLVED } \\
\text { (MG/I) }\end{array}$ & $\begin{array}{c}\text { NITRO- } \\
\text { GEN, } \\
\text { NO2+NO3 } \\
\text { TOTAL } \\
\text { (MG / L } \\
\text { AS N) }\end{array}$ & $\begin{array}{l}\text { NITRO- } \\
\text { GEN, } \\
\text { AMMONIA } \\
\text { TOTAL } \\
\text { (MG/L } \\
\text { AS N) }\end{array}$ & $\begin{array}{c}\text { PHOS- } \\
\text { PHORUS, } \\
\text { TOTAL } \\
\text { (MG/L } \\
\text { AS P) }\end{array}$ & $\begin{array}{l}\text { IRON, } \\
\text { DIS- } \\
\text { SOLVED } \\
\text { (UG/L } \\
\text { AS FF) }\end{array}$ & $\begin{array}{l}\text { MANGA- } \\
\text { NESE, } \\
\text { DIS- } \\
\text { SOLVED } \\
\text { (UG/L } \\
\text { AS MN) }\end{array}$ \\
\hline
\end{tabular}

\begin{tabular}{|c|c|c|c|c|c|c|c|c|}
\hline FEB & & & & & & & & \\
\hline $16 \ldots$ & 42 & 10 & 202 & .11 & .100 & .050 & 40 & 10 \\
\hline $16 \ldots$ & -- & - & -- & -- & -- & -- & - & - \\
\hline $16 \ldots$ & $36^{--}$ & $10^{--}$ & 178 & 13 & $1 \overline{0}$ & 040 & $\overline{40}$ & \\
\hline
\end{tabular}

301817096364101 SOMERVILLE LAKE SITE EC

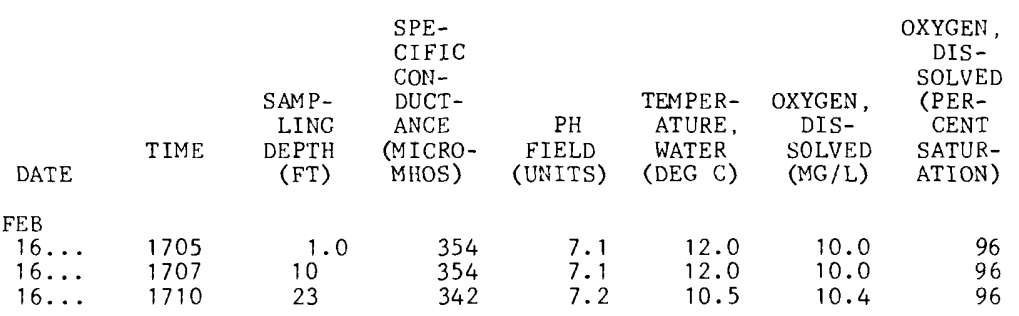

301754096380801 SOMERVILLE LAKE SITE FC

\begin{tabular}{|c|c|c|c|c|c|c|c|c|}
\hline DATE & TIME & $\begin{array}{l}\text { SAMP- } \\
\text { LING } \\
\text { DEPTH } \\
(\mathrm{FT})\end{array}$ & $\begin{array}{l}\text { SPE- } \\
\text { CIFIC } \\
\text { CON- } \\
\text { DUCT- } \\
\text { ANCE } \\
\text { (MICRO- } \\
\text { MHOS) }\end{array}$ & $\begin{array}{c}\text { PH } \\
\text { FIELD } \\
\text { (UNITS) }\end{array}$ & 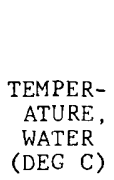 & $\begin{array}{l}\text { TRANS- } \\
\text { PAR- } \\
\text { ENCY } \\
\text { (SECCHI } \\
\text { DISK) } \\
\text { (M) }\end{array}$ & $\begin{array}{c}\text { OXYGEN, } \\
\text { DIS- } \\
\text { SOLVED } \\
\text { (MG/L) }\end{array}$ & $\begin{array}{c}\text { OXYGEN, } \\
\text { DIS- } \\
\text { SOLVED } \\
\text { (PER- } \\
\text { CENT } \\
\text { SATUR- } \\
\text { ATION) }\end{array}$ \\
\hline $\begin{array}{l}E B \\
16 \ldots \\
16 \ldots\end{array}$ & $\begin{array}{l}1720 \\
1725\end{array}$ & $14^{1.0}$ & $\begin{array}{l}313 \\
313\end{array}$ & $\begin{array}{l}6.8 \\
6.8\end{array}$ & $\begin{array}{l}13.0 \\
13.0\end{array}$ & .15 & $\begin{array}{l}8.5 \\
8.5\end{array}$ & $\begin{array}{l}83 \\
83\end{array}$ \\
\hline
\end{tabular}


Table 6--CHEMICAL-QUALITY SURVEYS OF SOMERVILLE LAKE FEBRUARY 16, 1977--Continued

301754096380801 SOMERVILLE LAKE SITE FC--Continued

\begin{tabular}{|c|c|c|c|c|c|c|c|c|c|}
\hline & $\begin{array}{l}\text { HARD- } \\
\text { NESS } \\
\text { (MG/L } \\
\text { AS }\end{array}$ & $\begin{array}{c}\text { HARD- } \\
\text { NESS, } \\
\text { NONCAR- } \\
\text { BONATE } \\
\text { (MG/L }\end{array}$ & $\begin{array}{l}\text { CALCIUM } \\
\text { DIS- } \\
\text { SOLVED } \\
\text { (MG/L }\end{array}$ & $\begin{array}{l}\text { MAGNE- } \\
\text { SIUM, } \\
\text { DIS- } \\
\text { SOLVED } \\
\text { (MG/L }\end{array}$ & $\begin{array}{l}\text { SODIUM, } \\
\text { DIS- } \\
\text { SOLVED } \\
\text { (MG/L }\end{array}$ & $\begin{array}{c}\text { SODIUM } \\
\text { AD- } \\
\text { SORP- } \\
\text { TION } \\
\text { RATIO }\end{array}$ & $\begin{array}{l}\text { POTAS- } \\
\text { SIUM, } \\
\text { DIS- } \\
\text { SOLVED } \\
\text { (MG/L }\end{array}$ & $\begin{array}{c}\text { BICAR- } \\
\text { BONATE } \\
\text { (MG/L } \\
\text { AS }\end{array}$ & $\begin{array}{l}\text { SULFATE } \\
\text { DIS- } \\
\text { SOLVED } \\
\text { (MG/L }\end{array}$ \\
\hline & $\mathrm{CACO} 3)$ & (ACO3) & AS $(A)$ & AS MG) & AS NA) & & AS $K)$ & $\mathrm{HCO} 3)$ & AS SO4) \\
\hline
\end{tabular}

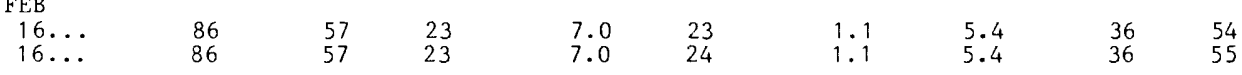

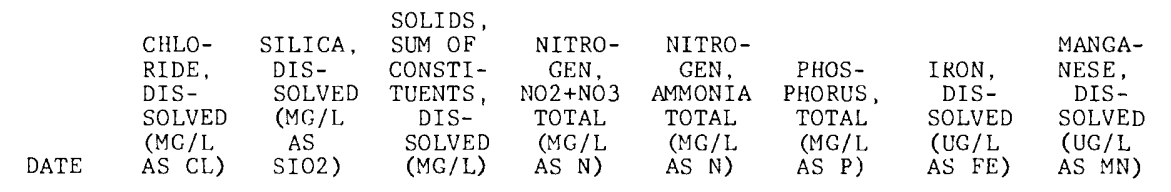

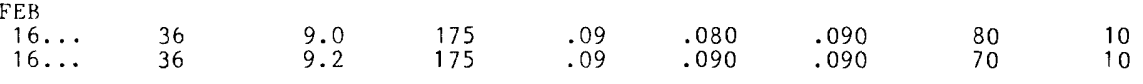




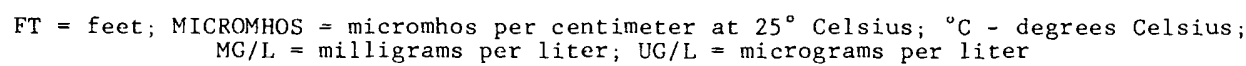

301908096313101 SOMERVILLE LAKE SITE AC

\begin{tabular}{|c|c|c|c|c|c|c|c|c|c|}
\hline DATE & TIME & $\begin{array}{l}\text { SAMP- } \\
\text { LING } \\
\text { DEPTH } \\
\text { (FT) }\end{array}$ & $\begin{array}{l}\text { SPE- } \\
\text { CIFIC } \\
\text { CON- } \\
\text { DUCT- } \\
\text { ANCE } \\
\text { (MICRO- } \\
\text { MHOS) }\end{array}$ & $\begin{array}{c}\text { PH } \\
\text { FIELD } \\
\text { (UNITS) }\end{array}$ & $\begin{array}{c}\text { TEMPER- } \\
\text { ATURE, } \\
\text { WATER } \\
\text { (DEG C) }\end{array}$ & $\begin{array}{c}\text { TRANS- } \\
\text { PAR- } \\
\text { ENCY } \\
\text { (SECCHI } \\
\text { DISK) } \\
(M)\end{array}$ & $\begin{array}{c}\text { OXYGEN, } \\
\text { DIS- } \\
\text { SOLVED } \\
\text { (MG/L) }\end{array}$ & $\begin{array}{c}\text { OXYGEN, } \\
\text { DIS- } \\
\text { SOLVED } \\
\text { (PER- } \\
\text { CENT } \\
\text { SATUR- } \\
\text { ATION) }\end{array}$ & $\begin{array}{l}\text { HARD- } \\
\text { NESS } \\
\text { (MG/L } \\
\text { AS } \\
\text { CACO3) }\end{array}$ \\
\hline $\begin{array}{r}\text { MAY } \\
27 \ldots \\
27 \ldots \\
27 \ldots \\
27 \ldots\end{array}$ & $\begin{array}{l}1025 \\
1028 \\
1030 \\
1035\end{array}$ & $\begin{array}{l}1.0 \\
10 \\
20 \\
31\end{array}$ & $\begin{array}{l}312 \\
312 \\
312 \\
312\end{array}$ & $\begin{array}{l}8.5 \\
8.2 \\
7.6 \\
7.3\end{array}$ & $\begin{array}{l}27.0 \\
26.5 \\
25.5 \\
24.5\end{array}$ & $\begin{array}{l}.70 \\
-- \\
-- \\
--\end{array}$ & $\begin{array}{l}7.8 \\
7.6 \\
6.4 \\
4.6\end{array}$ & $\begin{array}{l}99 \\
96 \\
80 \\
56\end{array}$ & $\begin{array}{l}89 \\
-- \\
-\overline{87}\end{array}$ \\
\hline DATE. & $\begin{array}{l}\text { HARD- } \\
\text { NESS, } \\
\text { NONCAR- } \\
\text { BONATE } \\
\text { (MG/L } \\
\text { CACO3) }\end{array}$ & $\begin{array}{l}\text { CALCIUM } \\
\text { DIS- } \\
\text { SOLVED } \\
\text { (MG/L } \\
\text { AS CA) }\end{array}$ & $\begin{array}{l}\text { MAGNE- } \\
\text { SIUM, } \\
\text { DIS- } \\
\text { SOLVED } \\
\text { (MC/L } \\
\text { AS MC) }\end{array}$ & $\begin{array}{l}\text { SODIUM, } \\
\text { DIS- } \\
\text { SOLVED } \\
\text { (MG/L } \\
\text { AS NA) }\end{array}$ & $\begin{array}{c}\text { SODIUM } \\
\text { AD- } \\
\text { SORP- } \\
\text { TION } \\
\text { RATIO }\end{array}$ & $\begin{array}{l}\text { POTAS- } \\
\text { SIUM, } \\
\text { DIS- } \\
\text { SOLVED } \\
\text { (MG/L } \\
\text { AS K) }\end{array}$ & $\begin{array}{c}\text { BICAR- } \\
\text { BONATE } \\
\text { (MG / L } \\
\text { AS } \\
\text { HCO3) }\end{array}$ & $\begin{array}{l}\text { SULFATE } \\
\text { DIS- } \\
\text { SOLVED } \\
\text { (MG/L } \\
\text { AS SO4) }\end{array}$ & $\begin{array}{l}\text { CHLO- } \\
\text { RIDE, } \\
\text { DIS- } \\
\text { SOLVED } \\
\text { (MG/L } \\
\text { AS CL) }\end{array}$ \\
\hline
\end{tabular}

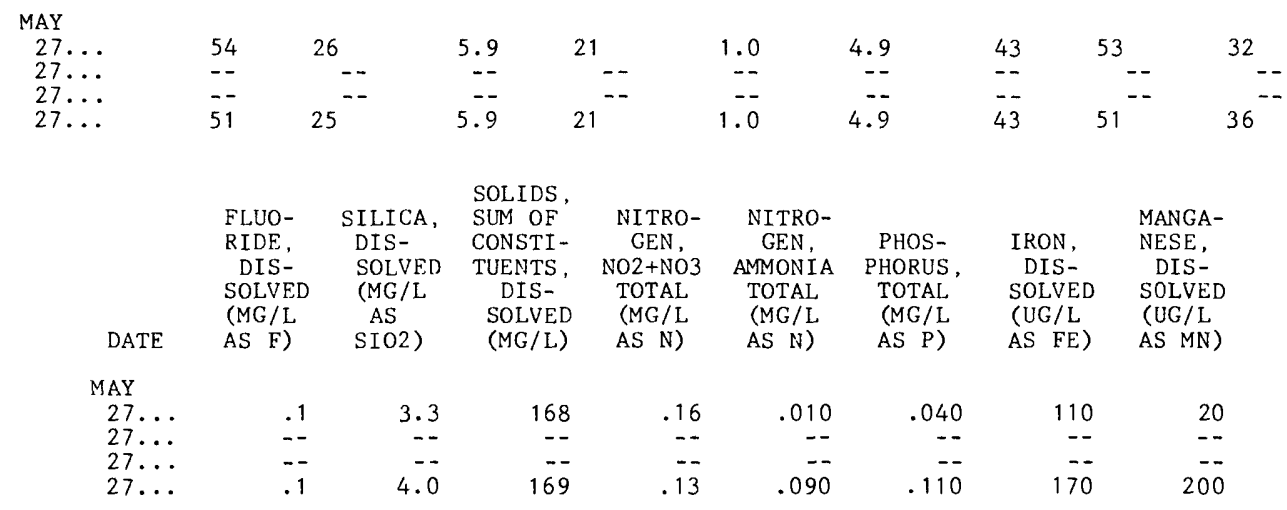

301940096315801 SOMERVILLE LAKE SITE AL

\begin{tabular}{|c|c|c|c|c|c|c|c|}
\hline DATE & TIME & $\begin{array}{l}\text { SAMP- } \\
\text { LING } \\
\text { DEPTH } \\
\text { (FT) }\end{array}$ & $\begin{array}{l}\text { SPE- } \\
\text { CIFIC } \\
\text { CON- } \\
\text { DUCT- } \\
\text { ANCE } \\
\text { (MICRO- } \\
\text { MHOS) }\end{array}$ & $\begin{array}{c}\text { PH } \\
\text { FIELD } \\
\text { (UNITS) }\end{array}$ & $\begin{array}{l}\text { TEMPER- } \\
\text { ATURE, } \\
\text { WATER } \\
\text { (DEG C) }\end{array}$ & $\begin{array}{c}\text { OXYGEN, } \\
\text { DIS- } \\
\text { SOLVED } \\
(M G / L)\end{array}$ & $\begin{array}{c}\text { OXYGEN, } \\
\text { DIS- } \\
\text { SOLVED } \\
\text { (PER- } \\
\text { CENT } \\
\text { SATUR- } \\
\text { ATION) }\end{array}$ \\
\hline $\begin{array}{r}\text { MAY } \\
27 \ldots \\
27 \ldots \\
27 \ldots \\
27 \ldots\end{array}$ & $\begin{array}{l}1012 \\
1014 \\
1016 \\
1018\end{array}$ & $\begin{array}{l}1.0 \\
10^{1.0} \\
20 \\
27\end{array}$ & $\begin{array}{l}312 \\
312 \\
312 \\
312\end{array}$ & $\begin{array}{l}8.5 \\
8.0 \\
7.6 \\
7.2\end{array}$ & $\begin{array}{l}27.0 \\
26.0 \\
25.0 \\
24.5\end{array}$ & $\begin{array}{l}7.7 \\
7.5 \\
6.4 \\
5.1\end{array}$ & $\begin{array}{l}97 \\
94 \\
79 \\
62\end{array}$ \\
\hline
\end{tabular}

302026096341501 SOMERVILLE LAKE SITE BC

\begin{tabular}{|c|c|c|c|c|c|c|c|}
\hline DATE & TIME & $\begin{array}{l}\text { SAMP- } \\
\text { LING } \\
\text { DEPTH } \\
\text { (FT) }\end{array}$ & $\begin{array}{l}\text { SPE- } \\
\text { CIFIC } \\
\text { CON- } \\
\text { DUCT- } \\
\text { ANCE } \\
\text { (MICRO- } \\
\text { MHOS) }\end{array}$ & $\begin{array}{c}\text { PH } \\
\text { FIELD } \\
\text { (UNITS) }\end{array}$ & $\begin{array}{c}\text { TEMPER- } \\
\text { ATURE, } \\
\text { WATER } \\
\left(\begin{array}{ll}\text { DEG }\end{array}\right.\end{array}$ & $\begin{array}{l}\text { TRANS- } \\
\text { PAR- } \\
\text { ENCY } \\
\text { (SECCHI } \\
\text { DISK) } \\
\text { (M) }\end{array}$ & $\begin{array}{c}\text { OXYGEN, } \\
\text { DIS- } \\
\text { SOLVED } \\
\text { (MG/L) }\end{array}$ \\
\hline $\begin{array}{l}\text { MAY } \\
27 \ldots \\
27 \ldots \\
27 \ldots\end{array}$ & $\begin{array}{l}1055 \\
1057 \\
1059\end{array}$ & $\begin{array}{l}1.0 \\
10^{17} \\
17\end{array}$ & $\begin{array}{l}308 \\
308 \\
308\end{array}$ & $\begin{array}{l}8.6 \\
7.3 \\
7.1\end{array}$ & $\begin{array}{l}28.5 \\
26.0 \\
25.0\end{array}$ & $\begin{array}{r}.64 \\
-- \\
--\end{array}$ & $\begin{array}{l}7.6 \\
5.6 \\
4.8\end{array}$ \\
\hline
\end{tabular}


Table 7--CHEMICAL-QUALITY SURVEYS OF SOMERVILLE LAKE MAY 27, 1977--Continued

302026096341501 SOMERVILLE LAKE SITE BC--Continued

\begin{tabular}{|c|c|c|c|c|c|c|}
\hline & $\begin{array}{l}\text { OXYGEN, } \\
\text { DIS- } \\
\text { SOLVED } \\
\text { (PER- } \\
\text { CENT } \\
\text { SATUR- }\end{array}$ & $\begin{array}{l}\text { NITRO- } \\
\text { GEN, } \\
\text { NO2+NO3 } \\
\text { TOTAL } \\
\text { (MG/L }\end{array}$ & $\begin{array}{l}\text { NITRO- } \\
\text { GEN, } \\
\text { AMMONIA } \\
\text { TOTAL } \\
\text { (MG/L }\end{array}$ & $\begin{array}{l}\text { PHOS- } \\
\text { PHORUS, } \\
\text { TOTAL } \\
\text { (MG/L }\end{array}$ & $\begin{array}{l}\text { IRON, } \\
\text { DIS- } \\
\text { SOLVED } \\
\text { (UG/L }\end{array}$ & $\begin{array}{l}\text { MANGA- } \\
\text { NESE, } \\
\text { DIS- } \\
\text { SOLVED } \\
\text { (UG / L }\end{array}$ \\
\hline $\mathrm{TT}$ & ATION) & AS N) & AS N) & AS P) & AS FE) & AS $M N$ ) \\
\hline
\end{tabular}

$\begin{array}{rrrrrrr}\text { MAY } & & & & \\ 27 \ldots & 99 & .06 & .010 & .040 & 70 & 20 \\ 27 \ldots & 70 & -\overline{-1} & .100 & .050 & 70 & 170 \\ 27 \ldots & 59 & .15 & .100\end{array}$

301805096332501 SOMERVILLE LAKE SITE CC

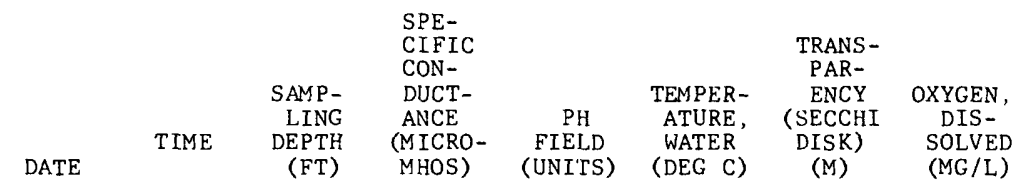

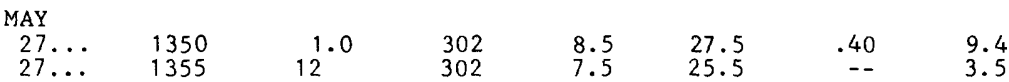

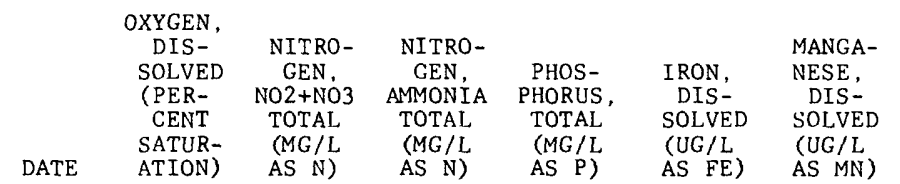

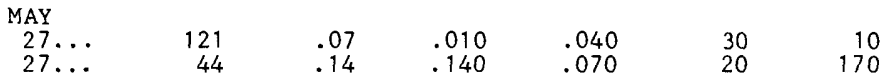

301847096334601 SOMERVILLE LAKE SITE DR

\begin{tabular}{|c|c|c|c|c|c|c|c|}
\hline & & & $\begin{array}{l}\text { SPE- } \\
\text { CIFIC } \\
\text { CON- }\end{array}$ & & & & $\begin{array}{c}\text { OXYGEN } \\
\text { DIS- } \\
\text { SOLVED }\end{array}$ \\
\hline DATE & T IME & $\begin{array}{r}\text { SAMP- } \\
\text { LING } \\
\text { DEPTH } \\
\text { (FT) }\end{array}$ & $\begin{array}{l}\text { DUCT- } \\
\text { ANCE } \\
\text { (MICRO- } \\
\text { MHOS) }\end{array}$ & $\begin{array}{c}\text { PH } \\
\text { FIELD } \\
\text { (UNITS) }\end{array}$ & $\begin{array}{c}\text { TFMPER- } \\
\text { ATURE, } \\
\text { WATER } \\
\text { (DEG C) }\end{array}$ & $\begin{array}{c}\text { OXYGEN, } \\
\text { DIS- } \\
\text { SOLVED } \\
\text { (MG/L) }\end{array}$ & $\begin{array}{c}\text { (PER- } \\
\text { CENT } \\
\text { SATUR- } \\
\text { ATION) }\end{array}$ \\
\hline
\end{tabular}

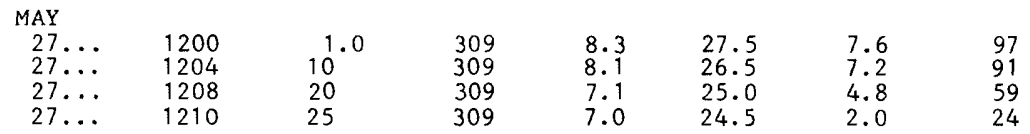

301904096335601 SOMERVILLE LAKE SITE DC

\begin{tabular}{|c|c|c|c|c|c|c|c|c|}
\hline DATE & TIME & $\begin{array}{l}\text { SAMP- } \\
\text { LING } \\
\text { DEPTH } \\
\text { (FT) }\end{array}$ & $\begin{array}{l}\text { SPE- } \\
\text { CIFIC } \\
\text { CON- } \\
\text { DUCT- } \\
\text { ANCE } \\
\text { (MICRO- } \\
\text { MHOS) }\end{array}$ & $\begin{array}{c}\text { PH } \\
\text { FIELD } \\
\text { (UNI'TS) }\end{array}$ & 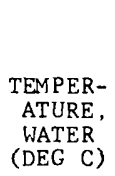 & $\begin{array}{l}\text { TRANS- } \\
\text { PAR- } \\
\text { ENCY } \\
\text { (SECCHI } \\
\text { DISK) } \\
\text { (M) }\end{array}$ & $\begin{array}{c}\text { OXYGEN, } \\
\text { DIS- } \\
\text { SOLVED } \\
\text { (MG/L) }\end{array}$ & $\begin{array}{l}\text { OXYGEN, } \\
\text { DIS- } \\
\text { SOLVED } \\
\text { (PER- } \\
\text { CENT } \\
\text { SATUR- } \\
\text { ATION) }\end{array}$ \\
\hline $\begin{array}{r}\text { MAY } \\
27 \ldots \\
27 \ldots \\
27 \ldots \\
27 \ldots \\
27 \ldots\end{array}$ & $\begin{array}{l}1125 \\
1128 \\
1132 \\
1134 \\
1136\end{array}$ & $\begin{array}{l}1.0 \\
10^{\circ} \\
20 \\
25 \\
30\end{array}$ & $\begin{array}{l}309 \\
309 \\
309 \\
309 \\
309\end{array}$ & $\begin{array}{l}8.3 \\
7.4 \\
7.3 \\
7.1 \\
7.0\end{array}$ & $\begin{array}{l}28.0 \\
26.5 \\
25.0 \\
25.0 \\
24.5\end{array}$ & $\begin{array}{c}.64 \\
-- \\
-- \\
--\end{array}$ & $\begin{array}{r}7.2 \\
6.2 \\
5.5 \\
3.7 \\
.8\end{array}$ & $\begin{array}{l}92 \\
78 \\
68 \\
46 \\
10\end{array}$ \\
\hline
\end{tabular}


Table 7--CHEMICAL-QUALITY SURVEYS OF SOMERVILLE LAKE MAY 27, 1977--Continued

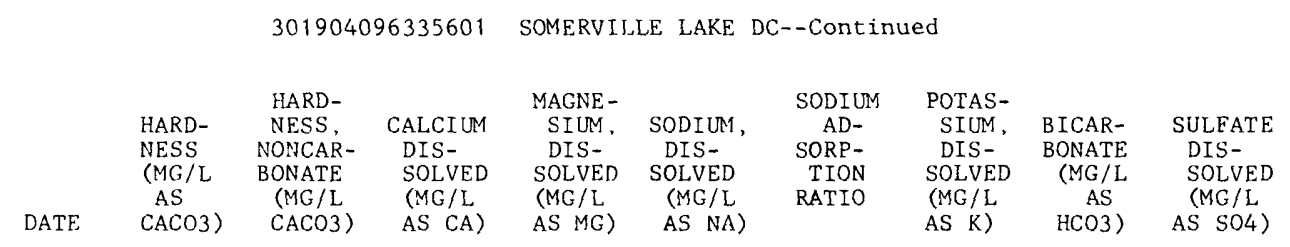

\begin{tabular}{|c|c|c|c|c|c|c|c|c|c|}
\hline \multicolumn{10}{|l|}{ MAY } \\
\hline $27 \ldots$ & -- & -- & -. & - & -- & -. & - & -- & -. \\
\hline $27 \ldots$ & -- & - & - & -. & -. & - & -- & -- & -- \\
\hline $27 \ldots$ & 87 & 51 & 25 & 5.9 & 21 & 1.0 & 4.9 & 44 & 52 \\
\hline $27 \ldots$ & -- & -- & -- & -- & -- & -- & -- & -- & -- \\
\hline DATE. & $\begin{array}{l}\text { CHLO- } \\
\text { RIDE, } \\
\text { DIS- } \\
\text { SOLVED } \\
\text { (MG/L } \\
\text { AS CL) }\end{array}$ & $\begin{array}{l}\text { SILICA, } \\
\text { DIS- } \\
\text { SOLVED } \\
\text { (MG/L } \\
\text { AS } \\
\text { SIO2) }\end{array}$ & $\begin{array}{c}\text { SOLIDS, } \\
\text { SUM OF } \\
\text { CONSTI- } \\
\text { TUENTS, } \\
\text { DIS- } \\
\text { SOLVED } \\
\text { (MG/L) }\end{array}$ & $\begin{array}{l}\text { NITRO- } \\
\text { GEN, } \\
\text { NO2+NO3 } \\
\text { TOTAL } \\
\text { (MG/L } \\
\text { AS N) }\end{array}$ & $\begin{array}{l}\text { NITRO- } \\
\text { GEN, } \\
\text { AMMONIA } \\
\text { TOTAL } \\
\text { (MG/L } \\
\text { AS N) }\end{array}$ & $\begin{array}{l}\text { PHOS- } \\
\text { PHORUS, } \\
\text { TOTAL } \\
\text { (MG/L } \\
\text { AS P) }\end{array}$ & $\begin{array}{l}\text { IRON, } \\
\text { DIS- } \\
\text { SOLVED } \\
\text { (UG/L } \\
\text { AS FE) }\end{array}$ & & \\
\hline
\end{tabular}

\begin{tabular}{|c|c|c|c|c|c|c|c|c|}
\hline \multicolumn{9}{|l|}{ MAY } \\
\hline $27 \ldots$ & 33 & 3.7 & 170 & .08 & .010 & .040 & 50 & 10 \\
\hline $27 \ldots$ & -- & - & -- & -- & $\cdots$ & - & -- & -- \\
\hline $27 \ldots$ & - & -. & -- & - & - & -- & -- & - \\
\hline $27 \ldots$ & 33 & 4.4 & 168 & .18 & .110 & .100 & 130 & 200 \\
\hline $27 \ldots$ & -- & -- & - & -- & -- & -- & - & -- \\
\hline
\end{tabular}

301817096364101 SOMERVILLE LAKE SITE EC

\begin{tabular}{|c|c|c|c|c|c|c|c|}
\hline & & & $\begin{array}{l}\text { SPE- } \\
\text { CIFIC } \\
\text { CON- } \\
\text { DUIC- }\end{array}$ & & & & $\begin{array}{c}\text { OXYGEN, } \\
\text { DIS- } \\
\text { SOLVED } \\
\text { (PER- }\end{array}$ \\
\hline DATE & TIME & $\begin{array}{c}\text { LING } \\
\text { DEPTH } \\
\text { (FT) }\end{array}$ & $\begin{array}{l}\text { ANCE } \\
\text { (MICRO- } \\
\text { MHOS) }\end{array}$ & $\begin{array}{c}\text { PH } \\
\text { FIELD } \\
\text { (UNITS) }\end{array}$ & $\begin{array}{l}\text { ATURE, } \\
\text { WATER } \\
\left(\begin{array}{lll}\text { DEG } & \text { C) }\end{array}\right.\end{array}$ & $\begin{array}{c}\text { OXYGEN, } \\
\text { DIS- } \\
\text { SOLVED } \\
(M G / L)\end{array}$ & $\begin{array}{l}\text { CENT- } \\
\text { CATUR- } \\
\text { ATION) }\end{array}$ \\
\hline \multicolumn{8}{|l|}{ MAY } \\
\hline $\begin{array}{l}27 \ldots \\
27 \ldots \\
27 \ldots\end{array}$ & $\begin{array}{l}1220 \\
1223 \\
1226\end{array}$ & $\begin{array}{l}1.0 \\
10 \\
23\end{array}$ & $\begin{array}{l}309 \\
309 \\
309\end{array}$ & $\begin{array}{l}8.8 \\
7.5 \\
7.2\end{array}$ & $\begin{array}{l}28.0 \\
25.5 \\
25.0\end{array}$ & $\begin{array}{l}7.8 \\
5.2 \\
3.1\end{array}$ & $\begin{array}{r}100 \\
65 \\
38\end{array}$ \\
\hline
\end{tabular}

301754096380801 SOMERVILLE LAKE SITE FC

\begin{tabular}{|c|c|c|c|c|c|c|c|c|c|c|c|c|}
\hline DATE & TIME & $\begin{array}{l}\text { SAMP- } \\
\text { LING } \\
\text { DEPTH } \\
(F T)\end{array}$ & $\begin{array}{l}\text { SPE- } \\
\text { CIFIC } \\
\text { CON- } \\
\text { DUCT- } \\
\text { ANCE } \\
\text { (MICRO- } \\
\text { MHOS) }\end{array}$ & $\begin{array}{c}\text { PH } \\
\text { FIELD } \\
\text { (UNITS) }\end{array}$ & $\begin{array}{c}\text { TEMPER- } \\
\text { ATURE, } \\
\text { WATER } \\
\left(\begin{array}{ll}\text { DEG } & \text { C) }\end{array}\right.\end{array}$ & $\begin{array}{c}\text { OXYGEN, } \\
\text { DIS- } \\
\text { SOLVED } \\
\text { (MG/L) }\end{array}$ & $\begin{array}{l}\text { OXYGEN, } \\
\text { DIS- } \\
\text { SOLVED } \\
\text { (PER- } \\
\text { CENT } \\
\text { SATUR- } \\
\text { ATION) }\end{array}$ & $\begin{array}{l}\text { HARD- } \\
\text { NESS } \\
\text { (MG/L } \\
\text { AS } \\
\text { CACO3) }\end{array}$ & $\begin{array}{l}\text { HARD- } \\
\text { NESS } \\
\text { NONCAR- } \\
\text { BONATE } \\
\text { (MC/L } \\
\text { CACO3) }\end{array}$ & $\begin{array}{l}\text { CALCIUN } \\
\text { DIS- } \\
\text { SOLVED } \\
\text { (MG/L } \\
\text { AS CA) }\end{array}$ & $\begin{array}{l}\text { MAGNE- } \\
\text { SIUM, } \\
\text { DIS- } \\
\text { SOLVED } \\
\text { (MG/L } \\
\text { AS MG) }\end{array}$ & $\begin{array}{l}\text { SODIUM, } \\
\text { DIS- } \\
\text { SOLVED } \\
\text { (MG/L } \\
\text { AS NA) }\end{array}$ \\
\hline \multicolumn{13}{|l|}{ MAY } \\
\hline $\begin{array}{l}27 \ldots \\
27 \ldots\end{array}$ & $\begin{array}{l}1240 \\
1245\end{array}$ & $15^{1.0}$ & $\begin{array}{l}399 \\
375\end{array}$ & $\begin{array}{l}8.1 \\
7.2\end{array}$ & $\begin{array}{l}28.5 \\
26.0\end{array}$ & $\begin{array}{l}6.8 \\
1.5\end{array}$ & $\begin{array}{l}88 \\
19\end{array}$ & $\begin{array}{l}120 \\
110\end{array}$ & $\begin{array}{l}67 \\
60\end{array}$ & $\begin{array}{l}33 \\
31\end{array}$ & $\begin{array}{l}8.0 \\
7.5\end{array}$ & $\begin{array}{l}27 \\
26\end{array}$ \\
\hline
\end{tabular}


Table 7--CHEMICAL-QUALITY SURVEYS OF SOMERVILLE LAKE MAY 27, 1977--Continued

301754096380801 SOMERVILLE LAKE SITE FC--Continued

\begin{tabular}{|c|c|c|c|c|c|c|c|c|c|c|c|c|}
\hline DATE & $\begin{array}{c}\text { SODIUM } \\
\text { AD- } \\
\text { SORP- } \\
\text { TION } \\
\text { RATIO }\end{array}$ & $\begin{array}{l}\text { POTAS- } \\
\text { SIUM } \\
\text { DIS- } \\
\text { SOLVED } \\
(M G / L \\
\text { AS K) }\end{array}$ & $\begin{array}{c}\text { BICAR- } \\
\text { BONATE } \\
\text { (MG/L } \\
\text { AS } \\
\mathrm{HCO} 3 \text { ) }\end{array}$ & $\begin{array}{l}\text { SULFATE } \\
\text { DIS- } \\
\text { SOLVED } \\
\text { (MG/L } \\
\text { AS SO4) }\end{array}$ & $\begin{array}{l}\text { CHLO- } \\
\text { RIDE, } \\
\text { DIS- } \\
\text { SOLVED } \\
\text { (MC/L } \\
\text { AS CL) }\end{array}$ & $\begin{array}{l}\text { SILICA. } \\
\text { DIS- } \\
\text { SOLVED } \\
\text { (MG/L } \\
\text { AS } \\
\text { SIO2) }\end{array}$ & $\begin{array}{l}\text { SOLIDS, } \\
\text { SUM OF } \\
\text { CONSTI- } \\
\text { TUENTS, } \\
\text { DIS- } \\
\text { SOLVED } \\
\text { (MG/L) }\end{array}$ & $\begin{array}{c}\text { NITRO- } \\
\text { GEN, } \\
\text { NO2+NO3 } \\
\text { TOTAL } \\
\text { (MG/L } \\
\text { AS N) }\end{array}$ & $\begin{array}{l}\text { NITRO- } \\
\text { GEN, } \\
\text { AMMONIA } \\
\text { TOTAL } \\
\text { (MG/L } \\
\text { AS N) }\end{array}$ & $\begin{array}{c}\text { PHOS- } \\
\text { PHORUS, } \\
\text { TOTAL } \\
\text { (MG / L } \\
\text { AS P) }\end{array}$ & $\begin{array}{l}\text { IRON, } \\
\text { DIS- } \\
\text { SOLVED } \\
\text { (UG / } \\
\text { AS FE) }\end{array}$ & $\begin{array}{l}\text { MANGA- } \\
\text { NESE, } \\
\text { DIS- } \\
\text { SOLVED } \\
\text { (UG/L } \\
\text { AS MN) }\end{array}$ \\
\hline \multirow[t]{3}{*}{$\begin{array}{l}\text { MAY } \\
27 \ldots \\
27 \ldots\end{array}$} & $\begin{array}{l}1.1 \\
1.1\end{array}$ & $\begin{array}{l}5.3 \\
5.3\end{array}$ & $\begin{array}{l}59 \\
59\end{array}$ & $\begin{array}{l}66 \\
62\end{array}$ & $\begin{array}{l}43 \\
41\end{array}$ & $\begin{array}{l}6.2 \\
7.1\end{array}$ & $\begin{array}{l}218 \\
209\end{array}$ & $\begin{array}{l}.01 \\
.03\end{array}$ & $\begin{array}{l}.010 \\
.250\end{array}$ & $\begin{array}{l}.070 \\
.090\end{array}$ & $\begin{array}{l}30 \\
70\end{array}$ & $\begin{array}{r}30 \\
430\end{array}$ \\
\hline & & DATE & TIME & $\begin{array}{l}\text { SAMP- } \\
\text { LING } \\
\text { DEPTH } \\
(\text { FT })\end{array}$ & $\begin{array}{l}\text { SPE- } \\
\text { CIFIC } \\
\text { CON- } \\
\text { DUCT- } \\
\text { ANCE } \\
\text { (MICRO- } \\
\text { MHOS) }\end{array}$ & $\begin{array}{c}\text { PH } \\
\text { FIELD } \\
\text { (UNITS) }\end{array}$ & $\begin{array}{l}\text { TEMPER- } \\
\text { ATURE, } \\
\text { WATER } \\
\left(\begin{array}{lll}\text { DEG } & \text { C) }\end{array}\right.\end{array}$ & $\begin{array}{l}\text { TRANS- } \\
\text { PAR- } \\
\text { ENCY } \\
\text { (SECCHI } \\
\text { DISK) } \\
\text { (M) }\end{array}$ & $\begin{array}{c}\text { OXYGEN, } \\
\text { DIS- } \\
\text { SOLVED } \\
\text { (MG / L ) }\end{array}$ & $\begin{array}{c}\text { OXYGEN, } \\
\text { DIS- } \\
\text { SOLVED } \\
\text { (PER- } \\
\text { CENT } \\
\text { SATUR- } \\
\text { ATION) }\end{array}$ & & \\
\hline & & $\begin{array}{l}\text { SEP } \\
30 \ldots \\
30 \ldots\end{array}$ & $\begin{array}{l}1250 \\
1255\end{array}$ & $10^{1.0}$ & $\begin{array}{l}398 \\
398\end{array}$ & $\begin{array}{l}8.3 \\
8.2\end{array}$ & $\begin{array}{l}28.0 \\
28.0\end{array}$ & .43 & $\begin{array}{l}7.7 \\
7.0\end{array}$ & $\begin{array}{l}99 \\
90\end{array}$ & & \\
\hline
\end{tabular}


$\mathrm{FT}=$ feet $;$ MICROMHOS $=$ micromhos per centimeter at $25^{\circ}$ Celsius; ${ }^{\circ} \mathrm{C}-$ degrees Celsius; $\mathrm{MG} / \mathrm{L}=$ milligrams per liter; UG/L = micrograms per liter

301908096313101 SOMERVILLE LAKE SITE AC

\begin{tabular}{|c|c|c|c|c|c|c|c|c|c|}
\hline DATE & TIME & $\begin{array}{c}\text { SAMP- } \\
\text { LING } \\
\text { DEPTH } \\
(\mathrm{FT})\end{array}$ & $\begin{array}{l}\text { SPE- } \\
\text { CIFIC } \\
\text { CON- } \\
\text { DUCT- } \\
\text { ANCE } \\
\text { (MICRO- } \\
\text { MHOS) }\end{array}$ & $\begin{array}{c}\text { PH } \\
\text { FIELD } \\
\text { (UNITS) }\end{array}$ & $\begin{array}{c}\text { TEMPER- } \\
\text { ATURE, } \\
\text { WATER } \\
\text { (DEG C) }\end{array}$ & $\begin{array}{c}\text { TRANS- } \\
\text { PAR- } \\
\text { ENCY } \\
\text { (SECCHI } \\
\text { DISK) } \\
(M)\end{array}$ & $\begin{array}{c}\text { OXYGEN, } \\
\text { DIS- } \\
\text { SOLVED } \\
\text { (MG/L) }\end{array}$ & $\begin{array}{c}\text { OXYGEN, } \\
\text { DIS- } \\
\text { SOLVED } \\
\text { (PER- } \\
\text { CENT } \\
\text { SATUR- } \\
\text { ATION) }\end{array}$ & $\begin{array}{l}\text { HARD- } \\
\text { NESS } \\
\text { (MG/L } \\
\text { AS } \\
\text { CACO3) }\end{array}$ \\
\hline $\begin{array}{l}\text { EP } \\
30 \ldots \\
30 \ldots \\
30 \ldots \\
30 \ldots\end{array}$ & $\begin{array}{l}1045 \\
1050 \\
1055 \\
1059\end{array}$ & $\begin{array}{l}1.0 \\
10^{\circ} \\
20 \\
27\end{array}$ & $\begin{array}{l}377 \\
377 \\
377 \\
377\end{array}$ & $\begin{array}{l}8.2 \\
8.1 \\
8.1 \\
7.9\end{array}$ & $\begin{array}{l}28.5 \\
28.5 \\
28.5 \\
28.5\end{array}$ & $\begin{array}{l}.79 \\
-- \\
--\end{array}$ & $\begin{array}{l}7.5 \\
7.3 \\
7.2 \\
6.6\end{array}$ & $\begin{array}{l}97 \\
95 \\
94 \\
86\end{array}$ & $\begin{array}{r}110 \\
-- \\
110\end{array}$ \\
\hline DATE & $\begin{array}{l}\text { HARD- } \\
\text { NESS, } \\
\text { NONCAR- } \\
\text { BONATE } \\
\text { (MG / L } \\
\text { CACO3) }\end{array}$ & $\begin{array}{l}\text { CALCIUM } \\
\text { DIS- } \\
\text { SOLVED } \\
\text { (MG/L } \\
\text { AS CA) }\end{array}$ & $\begin{array}{l}\text { MAGNE- } \\
\text { SIUM, } \\
\text { DIS- } \\
\text { SOLVED } \\
\text { (MG/L } \\
\text { AS MG) }\end{array}$ & $\begin{array}{l}\text { SODIUM, } \\
\text { DIS- } \\
\text { SOLVED } \\
\text { (MC/L } \\
\text { AS NA) }\end{array}$ & $\begin{array}{c}\text { SODIUM } \\
\text { AD- } \\
\text { SORP- } \\
\text { TION } \\
\text { RATIO }\end{array}$ & $\begin{array}{l}\text { POTAS- } \\
\text { SIUM, } \\
\text { DIS- } \\
\text { SOLVED } \\
\text { (MG/L } \\
\text { AS K) }\end{array}$ & $\begin{array}{c}\text { BICAR- } \\
\text { BONATE } \\
\text { (MG / L } \\
\text { AS } \\
\text { HCO3) }\end{array}$ & $\begin{array}{l}\text { SULFATE } \\
\text { DIS- } \\
\text { SOLVED } \\
\text { (MG/L } \\
\text { AS SO4) }\end{array}$ & $\begin{array}{l}\text { CHLO- } \\
\text { RIDE, } \\
\text { DIS- } \\
\text { SOLVED } \\
\text { (MG/L } \\
\text { AS CL) }\end{array}$ \\
\hline
\end{tabular}

\begin{tabular}{|c|c|c|c|c|c|c|c|c|c|}
\hline $\begin{array}{l}\text { SEP } \\
30 \ldots\end{array}$ & 59 & 30 & 7.6 & 26 & 1.1 & 5.9 & 57 & 63 & 43 \\
\hline $30 \ldots$ & -- & - & -- & -- & -- & - & -- & -- & -- \\
\hline $\begin{array}{l}30 \ldots \\
30 \ldots\end{array}$ & 59 & $30^{--}$ & $7 . \overline{5}$ & $27^{--}$ & 1.1 & 4.0 & $\overline{57}$ & $58^{--}$ & $44^{--}$ \\
\hline
\end{tabular}

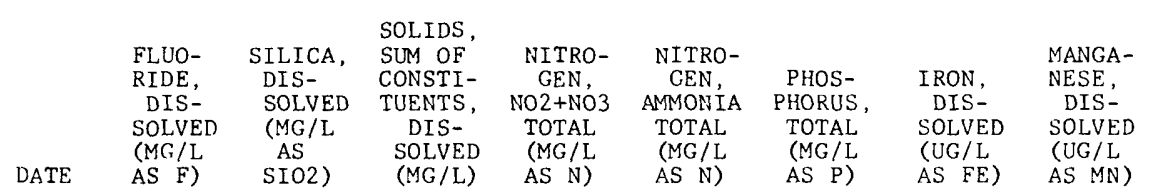

\begin{tabular}{|c|c|c|c|c|c|c|c|c|}
\hline \multicolumn{9}{|l|}{ SEP } \\
\hline $30 \ldots$ & .2 & 11 & 215 & .00 & .010 & .050 & 20 & 0 \\
\hline $30 \ldots$ & -- & -- & $\cdots$ & -- & -- & $=-$ & -- & -- \\
\hline $30 \ldots$ & -- & -- & - & .00 & .010 & .050 & 20 & 40 \\
\hline $30 \ldots$ & .3 & 8.0 & 207 & .00 & .010 & .060 & 20 & 40 \\
\hline
\end{tabular}

301940096315801 SOMERVILLE LAKE SITE AL

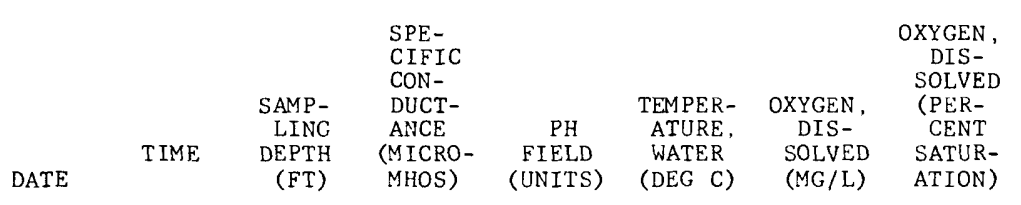

$\begin{array}{cccccccc}\text { SEP } & & & & & & \\ 30 \ldots & 1105 & 1.0 & 377 & 8.2 & 28.5 & 7.5 & 97 \\ 30 \ldots & 1110 & 10 & 377 & 8.2 & 28.5 & 7.4 & 96 \\ 30 \ldots & 1115 & 20 & 377 & 8.1 & 28.5 & 7.3 & 95\end{array}$

302026096341501 SOMERVILLE LAKE SITE BC

\begin{tabular}{ccccccc} 
SPE- & \multicolumn{3}{c}{$\begin{array}{c}\text { TRANS- } \\
\text { CIFIC } \\
\text { CON- }\end{array}$} \\
PAMP- \\
DUCT- \\
LING \\
DATE
\end{tabular}


Table 8--CHEMICAL-QUALITY SURVEYS OF SOMERVILLE LAKE SEPTEMBER 30, 1977--Continued

302026096341501 SOMERVILLE LAKE SITE BC--Continued

\begin{tabular}{|c|c|c|c|c|c|c|}
\hline DATE & $\begin{array}{l}\text { OXYGEN, } \\
\text { DIS- } \\
\text { SOLVED } \\
\text { (PER- } \\
\text { CENT } \\
\text { SATUR- } \\
\text { ATION) }\end{array}$ & $\begin{array}{c}\text { NITRO- } \\
\text { GEN } \\
\text { NO2+NO3 } \\
\text { TOTAL } \\
\text { (MG/L } \\
\text { AS N) }\end{array}$ & $\begin{array}{l}\text { NITRO- } \\
\text { GEN, } \\
\text { AMMONIA } \\
\text { TOTAL } \\
\text { (MG/L } \\
\text { AS N) }\end{array}$ & $\begin{array}{c}\text { PHOS- } \\
\text { PHORUS, } \\
\text { TOTAL } \\
\text { (MG/L } \\
\text { AS P) }\end{array}$ & $\begin{array}{l}\text { IRON, } \\
\text { DIS- } \\
\text { SOLVED } \\
\text { (UG/L } \\
\text { AS FE) }\end{array}$ & $\begin{array}{l}\text { MANGA- } \\
\text { NESE, } \\
\text { DIS- } \\
\text { SOLVED } \\
\text { (UG/L } \\
\text { AS MN) }\end{array}$ \\
\hline $\begin{array}{l}\text { SEP } \\
30 \ldots \\
30 \ldots\end{array}$ & $\begin{array}{l}96 \\
94\end{array}$ & $\begin{array}{l}.00 \\
.00\end{array}$ & $\begin{array}{l}.000 \\
.010\end{array}$ & $\begin{array}{l}.050 \\
.080\end{array}$ & $\begin{array}{r}20 \\
0\end{array}$ & $\begin{array}{l}20 \\
20\end{array}$ \\
\hline
\end{tabular}

301805096332501 SOMERVILLE LAKE SITE CC

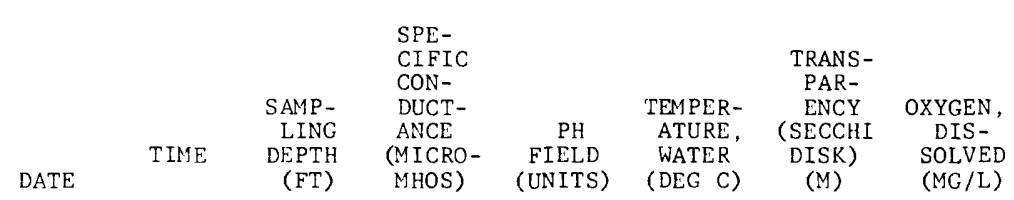

\begin{tabular}{|c|c|c|c|c|c|c|c|}
\hline SEP & & & & & & & \\
\hline $\begin{array}{l}30 \ldots \\
30 \ldots\end{array}$ & $\begin{array}{l}1315 \\
1320\end{array}$ & $12^{1 \cdot 0}$ & $\begin{array}{l}377 \\
377\end{array}$ & $\begin{array}{l}8.2 \\
7.9\end{array}$ & $\begin{array}{l}28.0 \\
28.0\end{array}$ & .58 & $\begin{array}{l}7.5 \\
6.9\end{array}$ \\
\hline
\end{tabular}

\begin{tabular}{ccccccc}
\multicolumn{7}{c}{ OXYGEN, } \\
DIS- & NITRO- & NITRO- & & & \\
& SOLVED & GEN, & GEN, & PHOS- & IRON, & MANGA- \\
& (PER- & NO2+NO3 & AMMONIA & PHORUS, & DIS- & DIS- \\
& CENT & TOTAL & TOTAL & TOTAL & SOLVED & SOLVED \\
& SATUR- & (MG/L & (MG/L & (MG/L & (UG/L & (UG/L \\
DATE & ATION) & AS N) & AS N) & AS P) & AS FE) & AS MN)
\end{tabular}

$\begin{array}{lllllll}\text { SEP } & & & & & \\ 30 \ldots & 96 & .01 & .010 & .050 & 10 & 0 \\ 30 \ldots & 88 & .00 & .010 & .080 & 10 & 0\end{array}$

301847096334601 SOMEKVILLE LAKE SITE DR

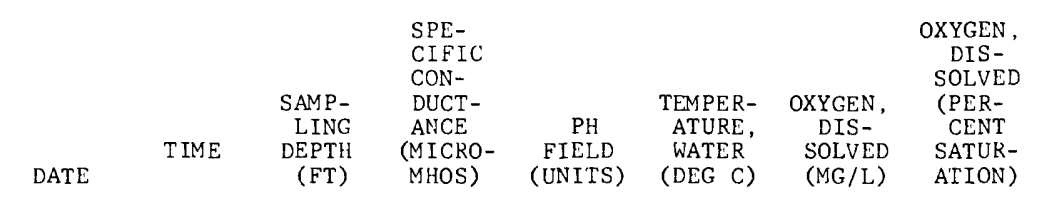

\begin{tabular}{|c|c|c|c|c|c|c|c|}
\hline \multicolumn{8}{|l|}{ SEP } \\
\hline $\begin{array}{l}30 \ldots \\
30 \ldots \\
30 \ldots\end{array}$ & $\begin{array}{l}1205 \\
1210 \\
1215\end{array}$ & $\begin{array}{l}1.0 \\
10^{\circ} \\
20\end{array}$ & $\begin{array}{l}382 \\
382 \\
382\end{array}$ & $\begin{array}{l}8.0 \\
8.0 \\
7.7\end{array}$ & $\begin{array}{l}28.0 \\
28.0 \\
28.0\end{array}$ & $\begin{array}{l}7.2 \\
7.0 \\
6.1\end{array}$ & $\begin{array}{l}92 \\
90 \\
78\end{array}$ \\
\hline
\end{tabular}

301904096335601 SOMERVILLE LAKE SITE DC

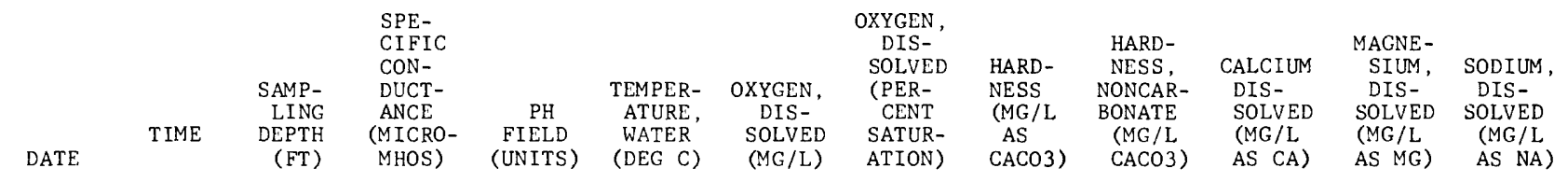

\begin{tabular}{|c|c|c|c|c|c|c|c|c|c|c|c|c|}
\hline \multicolumn{13}{|l|}{ SEP } \\
\hline $30 \ldots$ & 1150 & 1.0 & 382 & 8.3 & 28.0 & 7.7 & 99 & 110 & 59 & 30 & 7.8 & 27 \\
\hline & $\begin{array}{l}1153 \\
1157\end{array}$ & $\begin{array}{l}10 \\
20\end{array}$ & $\begin{array}{l}382 \\
382\end{array}$ & $\begin{array}{l}8.1 \\
8.1\end{array}$ & $\begin{array}{l}28.0 \\
28.0\end{array}$ & $\begin{array}{l}7.4 \\
7.2\end{array}$ & $\begin{array}{l}95 \\
92\end{array}$ & $\begin{array}{l}-- \\
--\end{array}$ & -- & -- & -- & -- \\
\hline $30 \ldots$ & 1159 & 25 & 382 & 8.1 & 28.5 & 7.0 & 91 & 110 & 61 & 31 & 7.6 & 25 \\
\hline
\end{tabular}


Table 8--CHEMICAL-QUALITY SURVEYS OF SOMERVILLE LAKE SEPTEMBER 30, 1977--Continued

301904096335601 SOMERVILLE LAKE SITE DC--Continued

\begin{tabular}{|c|c|c|c|c|c|c|c|c|c|c|c|c|}
\hline DATE & $\begin{array}{l}\text { SODIUM } \\
\text { AD- } \\
\text { SORP- } \\
\text { TION } \\
\text { RATIO }\end{array}$ & $\begin{array}{l}\text { POTAS- } \\
\text { SIUM, } \\
\text { DIS- } \\
\text { SOLVED } \\
\text { (MG/L } \\
\text { AS K) }\end{array}$ & $\begin{array}{c}\text { BICAR- } \\
\text { BONATE } \\
\text { (MG/L } \\
\text { AS } \\
\text { HCO3) }\end{array}$ & $\begin{array}{l}\text { SULFATE } \\
\text { DIS- } \\
\text { SOLVED } \\
\text { (MG/L } \\
\text { AS SO4) }\end{array}$ & $\begin{array}{l}\text { CHLO- } \\
\text { RIDE, } \\
\text { DIS- } \\
\text { SOLVED } \\
\text { (MG/L } \\
\text { AS CL) }\end{array}$ & $\begin{array}{l}\text { SILICA. } \\
\text { DIS- } \\
\text { SOLVED } \\
\text { (MG/L } \\
\text { AS } \\
\text { SIO2) }\end{array}$ & $\begin{array}{c}\text { SOLIDS, } \\
\text { SUM OF } \\
\text { CONSTI- } \\
\text { TUENTS, } \\
\text { DIS- } \\
\text { SOLVED } \\
\text { (MG/L) }\end{array}$ & $\begin{array}{l}\text { NITRO- } \\
\text { CEN, } \\
\text { NO2+NO3 } \\
\text { TOTAL } \\
\text { (MG /L } \\
\text { AS N) }\end{array}$ & $\begin{array}{c}\text { NITKO- } \\
\text { GEN, } \\
\text { AMMONIA } \\
\text { TOTAL } \\
(\text { MG /L } \\
\text { AS N) }\end{array}$ & $\begin{array}{c}\text { PHOS- } \\
\text { PHORUS, } \\
\text { TOTAL } \\
\text { (MG/L } \\
\text { AS P) }\end{array}$ & $\begin{array}{l}\text { IRON, } \\
\text { DIS- } \\
\text { SOLVED } \\
\text { (UG /L } \\
\text { AS FE) }\end{array}$ & $\begin{array}{l}\text { MANGA- } \\
\text { NESE, } \\
\text { DIS- } \\
\text { SOLVED } \\
\text { (UG/L } \\
\text { AS MN) }\end{array}$ \\
\hline$E P$ & & & & & & & & & & & & \\
\hline & 1.1 & 5.7 & 58 & 65 & 45 & 11 & 220 & .03 & .040 & .070 & 20 & 0 \\
\hline & -- & -- & -- & -- & -- & -- & - & -- & $=$ & -- & -- & - \\
\hline 30. & $1 . \overline{0}$ & $5 . \overline{9}$ & 58 & $54^{-}$ & $45^{\circ}$ & $11^{-}$ & 208 & $.0 \overline{1}$ & $.0 \overline{10}$ & .060 & $\overline{10}$ & $\overline{0}$ \\
\hline
\end{tabular}

301817096364101 SOMERVILLE LAKE SITE EC

\begin{tabular}{|c|c|c|c|c|c|c|c|}
\hline DATE & TIME & $\begin{array}{l}\text { SAMP- } \\
\text { LING } \\
\text { DEPTH } \\
(F T)\end{array}$ & $\begin{array}{l}\text { SPE- } \\
\text { CIFIC } \\
\text { CON- } \\
\text { DUCT- } \\
\text { ANCE } \\
\text { (MICRO- } \\
\text { MHOS) }\end{array}$ & $\begin{array}{c}\text { PH } \\
\text { FIELD } \\
\text { (UNITS) }\end{array}$ & $\begin{array}{l}\text { TEMPER- } \\
\text { ATURE. } \\
\text { WATER } \\
\text { (DEG C) }\end{array}$ & $\begin{array}{c}\text { OXYGEN, } \\
\text { DIS- } \\
\text { SOLVED } \\
\text { (MG/L) }\end{array}$ & $\begin{array}{c}\text { OXYGEN, } \\
\text { DIS- } \\
\text { SOLVED } \\
\text { (PER- } \\
\text { CENT } \\
\text { SATUR- } \\
\text { ATION) }\end{array}$ \\
\hline \multicolumn{8}{|l|}{ SEP } \\
\hline $\begin{array}{l}30 \ldots \\
30 \ldots \\
30 \ldots\end{array}$ & $\begin{array}{l}1230 \\
1235 \\
1240\end{array}$ & $\begin{array}{l}1.0 \\
10^{1.0} \\
21\end{array}$ & $\begin{array}{l}382 \\
382 \\
382\end{array}$ & $\begin{array}{l}7.9 \\
7.8 \\
7.6\end{array}$ & $\begin{array}{l}28.0 \\
28.0 \\
28.0\end{array}$ & $\begin{array}{l}7.2 \\
6.9 \\
6.3\end{array}$ & $\begin{array}{l}92 \\
88 \\
81\end{array}$ \\
\hline
\end{tabular}

301754096380801 SOMERVILLE LAKE SITE FC

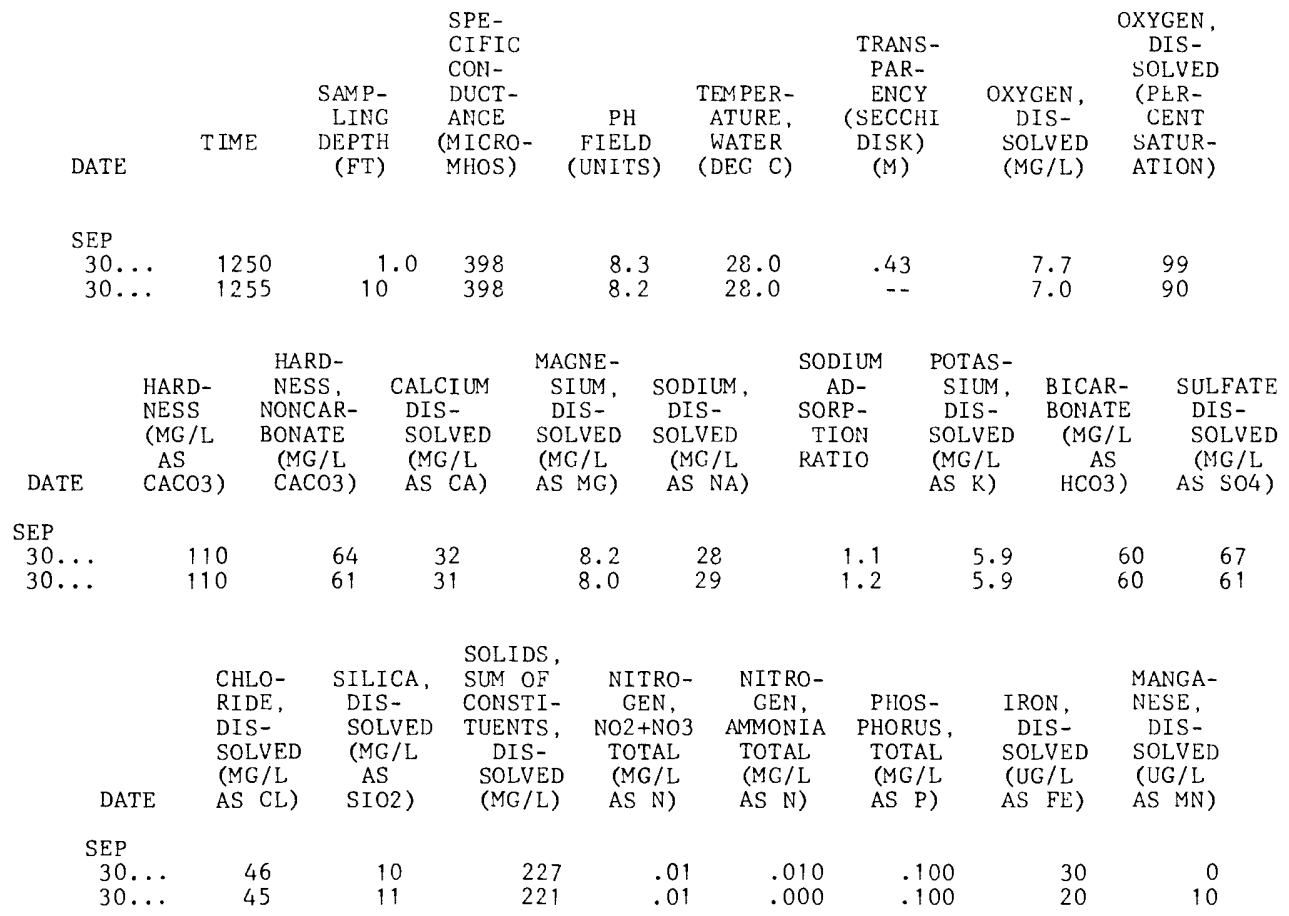


$\mathrm{FT}=$ feet $;$ MICROMHOS = micromhos per centimeter at $25^{\circ}$ Celsius; ${ }^{\circ} \mathrm{C}$ - degrees Celsius; $\mathrm{MG} / \mathrm{L}=$ milligrams per liter; UG/L = micrograms per liter

301908096313101 SOMERVILLE LAKE SITE AC

\begin{tabular}{|c|c|c|c|c|c|c|c|c|c|}
\hline DATE & TIME & $\begin{array}{l}\text { SAMP- } \\
\text { LING } \\
\text { DEPTH } \\
\text { (FT) }\end{array}$ & $\begin{array}{l}\text { SPE- } \\
\text { CIFIC } \\
\text { CON- } \\
\text { DUCT- } \\
\text { ANCE } \\
\text { (MICRO- } \\
\text { MHOS) }\end{array}$ & $\begin{array}{c}\text { PH } \\
\text { FIELD } \\
\text { (UNITS) }\end{array}$ & $\begin{array}{c}\text { TEMPER- } \\
\text { ATURE, } \\
\text { WATER } \\
\text { (DEG C) }\end{array}$ & $\begin{array}{l}\text { TRANS- } \\
\text { PAR- } \\
\text { ENCY } \\
\text { (SECCHI } \\
\text { DISK) } \\
\text { (M) }\end{array}$ & $\begin{array}{c}\text { OXYGEN, } \\
\text { DIS- } \\
\text { SOLVED } \\
\text { (MG/L) }\end{array}$ & $\begin{array}{c}\text { OXYGEN, } \\
\text { DIS- } \\
\text { SOLVED } \\
\text { (PER- } \\
\text { CENT } \\
\text { SATUR- } \\
\text { ATION) }\end{array}$ & $\begin{array}{l}\text { HARD- } \\
\text { NESS } \\
(\mathrm{MG} / \mathrm{L} \\
\mathrm{AS} \\
\mathrm{CACO} 3)\end{array}$ \\
\hline $\begin{array}{l}\text { MAR } \\
13 \ldots \\
13 \ldots \\
13 \ldots \\
13 \ldots\end{array}$ & $\begin{array}{l}1330 \\
1333 \\
1336 \\
1339\end{array}$ & $\begin{array}{r}1.0 \\
10.0 \\
20.0 \\
28.0\end{array}$ & $\begin{array}{l}401 \\
401 \\
401 \\
401\end{array}$ & $\begin{array}{l}8.2 \\
7.7 \\
7.6 \\
7.5\end{array}$ & $\begin{array}{l}15.0 \\
13.0 \\
12.5 \\
12.0\end{array}$ & $\begin{array}{l}.80 \\
-- \\
-- \\
--\end{array}$ & $\begin{array}{l}9.9 \\
9.0 \\
8.6 \\
7.5\end{array}$ & $\begin{array}{r}101 \\
88 \\
83 \\
72\end{array}$ & $\begin{array}{r}120 \\
-- \\
120\end{array}$ \\
\hline DATE & $\begin{array}{l}\text { HARD- } \\
\text { NESS } \\
\text { NONCAR- } \\
\text { BONATE } \\
\text { (MG/L } \\
\text { CACO3) }\end{array}$ & $\begin{array}{l}\text { CALCIUM } \\
\text { DIS- } \\
\text { SOLVED } \\
\text { (MG/L } \\
\text { AS CA) }\end{array}$ & $\begin{array}{l}\text { MAGNE- } \\
\text { SIUM, } \\
\text { DIS- } \\
\text { SOLVED } \\
\text { (MG/L } \\
\text { AS MG) }\end{array}$ & $\begin{array}{l}\text { SODIUM, } \\
\text { DIS- } \\
\text { SOLVED } \\
\text { (MG/L } \\
\text { AS NA) }\end{array}$ & $\begin{array}{c}\text { SODIUM } \\
\text { AD- } \\
\text { SORP- } \\
\text { TION } \\
\text { RATIO }\end{array}$ & $\begin{array}{l}\text { POTAS- } \\
\text { SIUM, } \\
\text { DIS- } \\
\text { SOLVED } \\
(\text { MG/L } \\
\text { AS K) }\end{array}$ & $\begin{array}{c}\text { BICAR- } \\
\text { BONATE } \\
\text { (MG /L } \\
\text { AS } \\
\text { HCO3) }\end{array}$ & $\begin{array}{c}\text { CAR- } \\
\text { BONATE } \\
\text { (MG/L } \\
\text { AS CO3) }\end{array}$ & $\begin{array}{l}\text { SULFATE } \\
\text { DIS- } \\
\text { SOLVED } \\
\text { (MG/I. } \\
\text { AS S04) }\end{array}$ \\
\hline
\end{tabular}

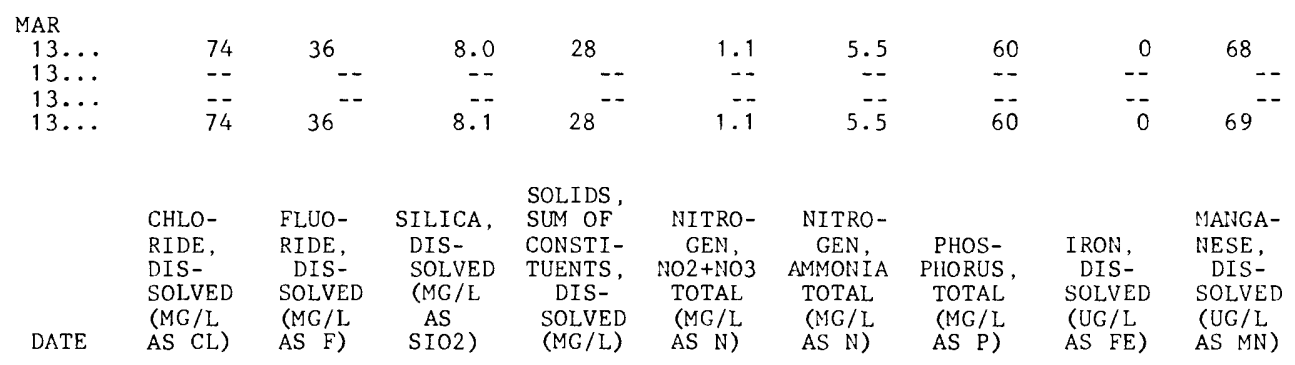

\begin{tabular}{|c|c|c|c|c|c|c|c|c|c|}
\hline $\begin{array}{c}\text { MAR } \\
13 \ldots\end{array}$ & 48 & .2 & 7.8 & 231 & .07 & .030 & .110 & 10 & 10 \\
\hline $13 \ldots$ & $=$ & -- & -- & -- & -- & -- & -- & -- & - \\
\hline $13 \ldots$ & -- & -- & $\cdots$ & -- & -- & -- & -- & - & $\ldots$ \\
\hline $13 \ldots$ & 50 & .2 & 7.9 & 234 & .08 & .050 & .100 & 10 & 0 \\
\hline
\end{tabular}

301940096315801 SOMERVILLE IJAKE SITE AL

\begin{tabular}{|c|c|c|c|c|c|c|c|}
\hline DATE & TIME & $\begin{array}{l}\text { SAMP- } \\
\text { LING } \\
\text { DEPTH } \\
\text { (FT) }\end{array}$ & $\begin{array}{l}\text { SPE- } \\
\text { CIFIC } \\
\text { CON- } \\
\text { DUCT- } \\
\text { ANCE } \\
\text { (MICRO- } \\
\text { MHOS) }\end{array}$ & $\begin{array}{c}\text { PH } \\
\text { FIELD } \\
\text { (UNI'TS) }\end{array}$ & $\begin{array}{l}\text { TEMPER- } \\
\text { ATURE, } \\
\text { WATER } \\
\left(\begin{array}{ll}\text { DEG } & \text { C) }\end{array}\right.\end{array}$ & $\begin{array}{c}\text { OXYGEN, } \\
\text { DIS- } \\
\text { SOLVED } \\
\text { (MG/L) }\end{array}$ & $\begin{array}{c}\text { OXYGEN, } \\
\text { DIS- } \\
\text { SOLVED } \\
\text { (PER- } \\
\text { CENT } \\
\text { SATUR- } \\
\text { ATION) }\end{array}$ \\
\hline MAR & & & & & & & \\
\hline $\begin{array}{l}13 \ldots \\
13 \ldots \\
13 \ldots \\
13 \ldots\end{array}$ & $\begin{array}{l}1350 \\
1353 \\
1356 \\
1358\end{array}$ & $\begin{array}{r}1.0 \\
10.0 \\
20.0 \\
27.0\end{array}$ & $\begin{array}{l}401 \\
401 \\
401 \\
401\end{array}$ & $\begin{array}{l}8.2 \\
7.9 \\
7.7 \\
7.5\end{array}$ & $\begin{array}{l}15.5 \\
13.0 \\
12.5 \\
12.5\end{array}$ & $\begin{array}{l}9.9 \\
9.4 \\
8.6 \\
8.4\end{array}$ & $\begin{array}{r}102 \\
92 \\
83 \\
82\end{array}$ \\
\hline
\end{tabular}

302026096341501 SOMERVILLE LAKE SITE BC

\begin{tabular}{|c|c|c|c|c|c|c|c|c|c|c|c|c|}
\hline DATE & T IME & $\begin{array}{c}\text { SAMP- } \\
\text { LING } \\
\text { DEPTH } \\
\text { (FT) }\end{array}$ & $\begin{array}{l}\text { SPE- } \\
\text { CIFIC } \\
\text { CON- } \\
\text { DUCT- } \\
\text { ANCE } \\
\text { (MICRO- } \\
\text { MHOS) }\end{array}$ & $\begin{array}{c}\text { PH } \\
\text { FIELD } \\
\text { (UNITS) }\end{array}$ & 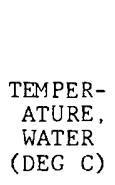 & $\begin{array}{c}\text { OXYGEN, } \\
\text { DIS- } \\
\text { SOLVED } \\
(\mathrm{MG} / \mathrm{L})\end{array}$ & $\begin{array}{c}\text { OXYGEN, } \\
\text { DIS- } \\
\text { SOLVED } \\
\text { (PER- } \\
\text { CENT } \\
\text { SATUR- } \\
\text { ATION) }\end{array}$ & $\begin{array}{c}\text { NITRO- } \\
\text { GEN } \\
\text { NO2+NO3 } \\
\text { TOTAL } \\
\text { (MG/L } \\
\text { AS N) }\end{array}$ & $\begin{array}{l}\text { NITRO- } \\
\text { GEN, } \\
\text { AMMONIA } \\
\text { TOTAL } \\
\text { (MG/L } \\
\text { AS N) }\end{array}$ & $\begin{array}{l}\text { PHOS- } \\
\text { PHORUS, } \\
\text { TOTAL } \\
\text { (MG/L } \\
\text { AS P) }\end{array}$ & $\begin{array}{l}\text { IRON, } \\
\text { DIS- } \\
\text { SOLVED } \\
\text { (UG/L } \\
\text { AS FE) }\end{array}$ & $\begin{array}{l}\text { MAINGA- } \\
\text { NLSE, } \\
\text { DIS- } \\
\text { SOLVED } \\
\text { (UG/L } \\
\text { AS MN) }\end{array}$ \\
\hline MAR & & & & & & & & & & & & \\
\hline $\begin{array}{l}13 \ldots \\
13 \ldots\end{array}$ & $\begin{array}{l}1405 \\
1408\end{array}$ & $\begin{array}{r}1.0 \\
13.0\end{array}$ & $\begin{array}{l}398 \\
398\end{array}$ & $\begin{array}{l}8.3 \\
7.9\end{array}$ & $\begin{array}{l}14.5 \\
13.5\end{array}$ & $\begin{array}{l}9.7 \\
8.9\end{array}$ & $\begin{array}{l}98 \\
88\end{array}$ & $\begin{array}{l}.02 \\
.06\end{array}$ & $\begin{array}{l}.000 \\
.010\end{array}$ & $\begin{array}{l}.100 \\
.100\end{array}$ & $\begin{array}{l}10 \\
10\end{array}$ & $\begin{array}{r}10 \\
0\end{array}$ \\
\hline
\end{tabular}


Table 9--CHFMICAL-QUALITY SURVEYS OF SOMERVILLE LAKE MARCH 13, 1978--Continued

301805096332501 SOMERVILLE LAKE SITE CC

\begin{tabular}{|c|c|c|c|c|c|c|c|}
\hline DATE & TIME & $\begin{array}{c}\text { SAMP- } \\
\text { LING } \\
\text { DEPTH } \\
\text { (FT) }\end{array}$ & $\begin{array}{l}\text { SPE- } \\
\text { CIFIC } \\
\text { CON- } \\
\text { DUCT- } \\
\text { ANCE } \\
\text { (MICRO- } \\
\text { MHOS) }\end{array}$ & $\begin{array}{c}\text { PH } \\
\text { FIELD } \\
\text { (UNITS) }\end{array}$ & $\begin{array}{l}\text { TEMPER- } \\
\text { ATURE, } \\
\text { WATER } \\
\text { (DEG C) }\end{array}$ & $\begin{array}{c}\text { TRANS- } \\
\text { PAR- } \\
\text { ENCY } \\
\text { (SECCHI } \\
\text { DISK) } \\
\text { (M) }\end{array}$ & $\begin{array}{c}\text { OXYGEN, } \\
\text { DIS- } \\
\text { SOLVED } \\
\text { (MG/L) }\end{array}$ \\
\hline \multicolumn{8}{|l|}{ AAR } \\
\hline & 1550 & 1.0 & 401 & 8. & 13. & .70 & 9.6 \\
\hline & 555 & 14.0 & 401 & 7.7 & 12.0 & -- & 7.9 \\
\hline
\end{tabular}

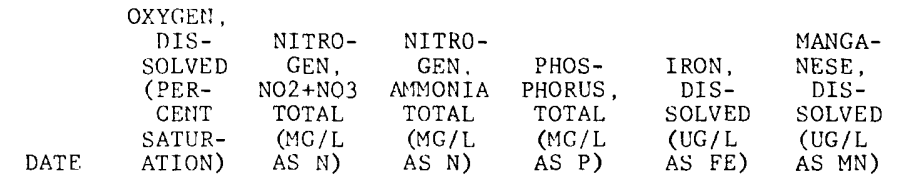

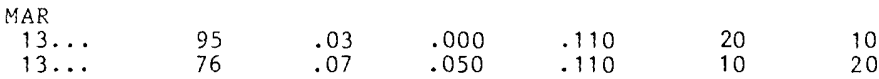

301847096334601 SOMERVILLE LAKE SITE DR

\begin{tabular}{|c|c|c|c|c|c|c|c|}
\hline & & & $\begin{array}{l}\text { SPE- } \\
\text { CIFIC } \\
\text { CON- }\end{array}$ & & & & $\begin{array}{c}\text { OXYGEN, } \\
\text { DIS- } \\
\text { SOLVED }\end{array}$ \\
\hline DATE & TIME & $\begin{array}{l}\text { SAMP- } \\
\text { LINC } \\
\text { DEPTH } \\
\text { (FT) }\end{array}$ & $\begin{array}{l}\text { DUCT- } \\
\text { ANCE } \\
\text { (MICRO- } \\
\text { MHOS) }\end{array}$ & $\begin{array}{c}\text { PH } \\
\text { FIELD } \\
\text { (UNITS) }\end{array}$ & $\begin{array}{c}\text { TEMPLR- } \\
\text { ATURE, } \\
\text { WATER } \\
\text { (DEG C) }\end{array}$ & $\begin{array}{c}\text { OXYGEN, } \\
\text { DIS- } \\
\text { SOLVED } \\
\text { (MG/L) }\end{array}$ & $\begin{array}{l}\text { (PER- } \\
\text { CENT } \\
\text { SATUR- } \\
\text { ATION) }\end{array}$ \\
\hline \multicolumn{8}{|l|}{ MAR } \\
\hline $\begin{array}{l}13 \ldots \\
13 \ldots \\
13 \ldots\end{array}$ & $\begin{array}{l}1450 \\
1452 \\
1455\end{array}$ & $\begin{array}{r}1.0 \\
10.0 \\
23.0\end{array}$ & $\begin{array}{l}401 \\
401 \\
401\end{array}$ & $\begin{array}{l}8.5 \\
7.9 \\
7.7\end{array}$ & $\begin{array}{l}14.5 \\
12.5 \\
12.0\end{array}$ & $\begin{array}{r}10.1 \\
9.1 \\
7.9\end{array}$ & $\begin{array}{r}102 \\
88 \\
76\end{array}$ \\
\hline
\end{tabular}

301904096335601 SOMERVILLE LAKE SITE DC

\begin{tabular}{|c|c|c|c|c|c|c|c|c|c|}
\hline DATE & TIME & $\begin{array}{r}\text { SAMP- } \\
\text { LING } \\
\text { DEPTH } \\
\text { (FT) }\end{array}$ & $\begin{array}{l}\text { SPE- } \\
\text { CIFIC } \\
\text { CON- } \\
\text { DUCT- } \\
\text { ANCE } \\
\text { (MICRO- } \\
\text { MHOS) }\end{array}$ & $\begin{array}{c}\text { PH } \\
\text { FIELD } \\
\text { (UNITS) }\end{array}$ & $\begin{array}{c}\text { TEMPER- } \\
\text { ATURE, } \\
\text { WATER } \\
\text { (DEC C) }\end{array}$ & $\begin{array}{c}\text { TRANS- } \\
\text { PAR- } \\
\text { ENCY } \\
\text { (SECCHI } \\
\text { DISK) } \\
\text { (M) }\end{array}$ & $\begin{array}{c}\text { OXYGEN, } \\
\text { DIS- } \\
\text { SOLVED } \\
\text { (MG/L) }\end{array}$ & $\begin{array}{c}\text { OXYGEN, } \\
\text { DIS- } \\
\text { SOLVED } \\
\text { (PER- } \\
\text { CENT } \\
\text { SATUR- } \\
\text { ATION) }\end{array}$ & $\begin{array}{l}\text { HARD- } \\
\text { NESS } \\
\text { (MG/L } \\
\text { AS } \\
\text { CACO3) }\end{array}$ \\
\hline $\begin{array}{l}\text { MAR } \\
13 \ldots \\
13 \ldots \\
13 \ldots \\
13 \ldots\end{array}$ & $\begin{array}{l}1430 \\
1433 \\
1436 \\
1439\end{array}$ & $\begin{array}{r}1.0 \\
10.0 \\
20.0 \\
27.0\end{array}$ & $\begin{array}{l}401 \\
401 \\
401 \\
401\end{array}$ & $\begin{array}{l}8.3 \\
8.0 \\
7.8 \\
7.7\end{array}$ & $\begin{array}{l}14.5 \\
12.5 \\
12.5 \\
12.5\end{array}$ & $\begin{array}{c}.80 \\
-- \\
-- \\
--\end{array}$ & $\begin{array}{r}10.1 \\
9.4 \\
9.1 \\
8.0\end{array}$ & $\begin{array}{r}102 \\
91 \\
88 \\
78\end{array}$ & $\begin{array}{r}120 \\
-- \\
130\end{array}$ \\
\hline DATE & $\begin{array}{l}\text { HARD- } \\
\text { NESS, } \\
\text { NONCAR- } \\
\text { BONATE } \\
\text { (MG/L } \\
\text { CACO3) }\end{array}$ & $\begin{array}{l}\text { CALCIUM } \\
\text { DIS- } \\
\text { SOLVED } \\
\text { (MG /L } \\
\text { AS CA) }\end{array}$ & $\begin{array}{l}\text { MAGNE- } \\
\text { SIUM, } \\
\text { DIS- } \\
\text { SOLVED } \\
\text { (MG / L } \\
\text { AS MG) }\end{array}$ & $\begin{array}{l}\text { SODIUM, } \\
\text { DIS- } \\
\text { SOLVED } \\
\text { (MG/L } \\
\text { AS NA) }\end{array}$ & $\begin{array}{c}\text { SODIUM } \\
\text { AD- } \\
\text { SORP- } \\
\text { TION } \\
\text { RATIO }\end{array}$ & $\begin{array}{l}\text { POTAS- } \\
\text { SIUM, } \\
\text { DIS- } \\
\text { SOLVED } \\
\text { (MG/L } \\
\text { AS K) }\end{array}$ & $\begin{array}{c}\text { BICAR- } \\
\text { BONATE } \\
\text { (MG / L } \\
\text { AS } \\
\text { HCO3) }\end{array}$ & $\begin{array}{c}\text { CAR- } \\
\text { BONATE } \\
(M G / L \\
\text { AS } \mathrm{CO} 3)\end{array}$ & $\begin{array}{l}\text { SULFATE } \\
\text { DIS- } \\
\text { SOLVED } \\
\text { (MG/L } \\
\text { AS SO4) }\end{array}$ \\
\hline
\end{tabular}

MAR

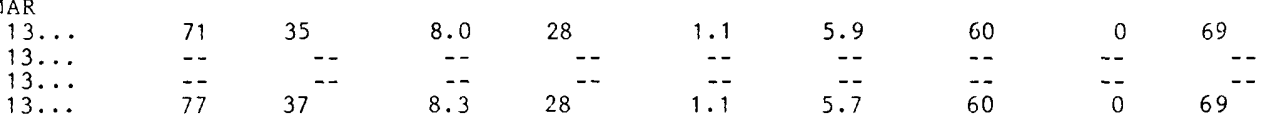


Table 9--CHEMICAL-QUALITY SURVEYS OF SOMERVILLE LAKE MARCH 13, 1978--Continued

301904096335601 SOMERVILLE LAKE SITE DC--Continued

\begin{tabular}{|c|c|c|c|c|c|c|c|c|}
\hline & $\begin{array}{l}\text { CHLO- } \\
\text { RIDE, } \\
\text { DIS- } \\
\text { SOLVED } \\
\text { (MG/L } \\
\text { AS CL) }\end{array}$ & $\begin{array}{l}\text { SILICA, } \\
\text { DIS- } \\
\text { SOLVED } \\
\text { (MG/L } \\
\text { AS } \\
\text { SIO2) }\end{array}$ & $\begin{array}{l}\text { SOLIDS } \\
\text { SUM OF } \\
\text { CONSTI- } \\
\text { TUENTS, } \\
\text { DIS- } \\
\text { SOLVED } \\
\text { (MG/L) }\end{array}$ & $\begin{array}{c}\text { NITRO- } \\
\text { GEN, } \\
\text { NO2+NO3 } \\
\text { TOTAL } \\
\text { (MG/L } \\
\text { AS N) }\end{array}$ & $\begin{array}{l}\text { NITRO- } \\
\text { GEN, } \\
\text { AMMONIA } \\
\text { TOTAL } \\
\text { (MG/L } \\
\text { AS N) }\end{array}$ & $\begin{array}{l}\text { PHOS- } \\
\text { PHORUS, } \\
\text { TOTAL } \\
\text { (MG/L } \\
\text { AS P) }\end{array}$ & $\begin{array}{l}\text { IRON, } \\
\text { DIS- } \\
\text { SOLVED } \\
\text { (UG/L } \\
\text { AS FE) }\end{array}$ & $\begin{array}{l}\text { MANGA- } \\
\text { NESE , } \\
\text { DIS- } \\
\text { SOLVED } \\
\text { (UG/L } \\
\text { AS MN) }\end{array}$ \\
\hline E & AS CL) & SIO2) & $(M G / L$ & & AS N) & AS P) & & $(\mathrm{MN})$ \\
\hline
\end{tabular}

\begin{tabular}{|c|c|c|c|c|c|c|c|c|}
\hline MAR & & & & & & & & \\
\hline $13 \ldots$ & 48 & 7.8 & 231 & .04 & .010 & .110 & 10 & 10 \\
\hline $13 \ldots$ & -- & -- & -- & -- & -- & -- & -- & - \\
\hline $13 \ldots$ & $48^{--}$ & $7-\overline{9}$ & 234 & $\overline{0}$ & 060 & $1 \overline{-}$ & $\overline{20}$ & $-\overline{20}$ \\
\hline
\end{tabular}

301817096364101 SOMERVILLE LAKE SITE EC

\begin{tabular}{|c|c|c|c|c|c|c|c|}
\hline DATE & TIME & $\begin{array}{l}\text { SAMP- } \\
\text { LING } \\
\text { DEPTH } \\
\text { (FT) }\end{array}$ & $\begin{array}{l}\text { SPE- } \\
\text { CIFIC } \\
\text { CON- } \\
\text { DUCT- } \\
\text { ANCE } \\
\text { (MICRO- } \\
\text { MHOS) }\end{array}$ & $\begin{array}{c}\text { PH } \\
\text { FIELD } \\
\text { (UNITS) }\end{array}$ & $\begin{array}{l}\text { TEMPER- } \\
\text { ATURE } \\
\text { WATER } \\
\text { (DEG } \quad \text { C) }\end{array}$ & $\begin{array}{c}\text { OXYGEN, } \\
\text { DIS- } \\
\text { SOLVED } \\
(M G / L)\end{array}$ & $\begin{array}{c}\text { OXYCEN, } \\
\text { DIS- } \\
\text { SOLVED } \\
\text { (PER- } \\
\text { CENT } \\
\text { SATUR- } \\
\text { ATION) }\end{array}$ \\
\hline \multicolumn{8}{|l|}{ MAR } \\
\hline $\begin{array}{l}13 \ldots \\
13 \ldots \\
13 \ldots \\
13 \ldots\end{array}$ & $\begin{array}{l}1505 \\
1507 \\
1509 \\
1510\end{array}$ & $\begin{array}{l}1.0 \\
10.0 \\
20.0 \\
25.0\end{array}$ & $\begin{array}{l}411 \\
411 \\
411 \\
411\end{array}$ & $\begin{array}{l}8.4 \\
7.9 \\
7.8 \\
7.8\end{array}$ & $\begin{array}{l}14.0 \\
12.5 \\
12.5 \\
12.5\end{array}$ & $\begin{array}{r}10.1 \\
9.0 \\
8.6 \\
8.5\end{array}$ & $\begin{array}{r}101 \\
87 \\
83 \\
83\end{array}$ \\
\hline
\end{tabular}

301754096380801 SOMERVILLE LAAE SITE FC

\begin{tabular}{|c|c|c|c|c|c|c|c|c|c|}
\hline DATE & TIME & $\begin{array}{l}\text { SAMP- } \\
\text { LING } \\
\text { DEPTH } \\
(\text { FT })\end{array}$ & $\begin{array}{l}\text { SPE- } \\
\text { CIFIC } \\
\text { CON- } \\
\text { DUCT- } \\
\text { ANCE } \\
\text { (MICRO- } \\
\text { MHOS) }\end{array}$ & $\begin{array}{c}\text { PH } \\
\text { FIELD } \\
\text { (UNI'TS) }\end{array}$ & 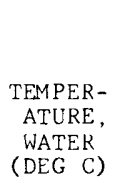 & $\begin{array}{l}\text { TRANS- } \\
\text { PAR- } \\
\text { ENCY } \\
\text { (SECCHI } \\
\text { DISK) } \\
(M)\end{array}$ & $\begin{array}{c}\text { OXYGEN, } \\
\text { DIS- } \\
\text { SOLVED } \\
\text { (MG/L) }\end{array}$ & $\begin{array}{c}\text { OXYGEN, } \\
\text { DIS- } \\
\text { SOLVED } \\
\text { (PER- } \\
\text { CENT } \\
\text { SATUR- } \\
\text { ATION) }\end{array}$ & $\begin{array}{l}\text { HARD- } \\
\text { NESS } \\
\text { (MG/L } \\
\text { AS } \\
\text { CACO3) }\end{array}$ \\
\hline $\begin{array}{l}\text { MAR } \\
13 \ldots \\
13 \ldots\end{array}$ & $\begin{array}{l}1525 \\
1529\end{array}$ & $\begin{array}{r}1.0 \\
13.0\end{array}$ & $\begin{array}{l}568 \\
575\end{array}$ & $\begin{array}{l}8.1 \\
7.5\end{array}$ & $\begin{array}{l}15.0 \\
14.0\end{array}$ & $\begin{array}{r}.40 \\
--\end{array}$ & $\begin{array}{l}9.4 \\
7.9\end{array}$ & $\begin{array}{l}96 \\
79\end{array}$ & $\begin{array}{l}180 \\
180\end{array}$ \\
\hline DATE & $\begin{array}{l}\text { HARD- } \\
\text { NESS } \\
\text { NONCAR- } \\
\text { BONATE } \\
\text { (MG/L } \\
\text { CACO3) }\end{array}$ & $\begin{array}{l}\text { CALCIUM } \\
\text { DIS- } \\
\text { SOLVED } \\
\text { (MG/L } \\
\text { AS CA) }\end{array}$ & $\begin{array}{c}\text { MAGNE- } \\
\text { SIUM, } \\
\text { DIS- } \\
\text { SOLVED } \\
\text { (MG/L } \\
\text { AS MG) }\end{array}$ & $\begin{array}{l}\text { SODIUM, } \\
\text { DIS- } \\
\text { SOLVED } \\
\text { (MG/L } \\
\text { AS NA) }\end{array}$ & $\begin{array}{c}\text { SODIUM } \\
\text { AD- } \\
\text { SORP- } \\
\text { TION } \\
\text { RATIO }\end{array}$ & $\begin{array}{l}\text { POTAS- } \\
\text { SIUM, } \\
\text { DIS- } \\
\text { SOLVED } \\
(M C / L \\
\text { AS K) }\end{array}$ & $\begin{array}{c}\text { BICAR- } \\
\text { BONATE } \\
\text { (MG } / \mathrm{L} \\
\text { AS } \\
\mathrm{HCO} 3)\end{array}$ & $\begin{array}{c}\text { CAR- } \\
\text { BONATE } \\
\text { (MG/L } \\
\text { AS CO3) }\end{array}$ & $\begin{array}{c}\text { SULFATE } \\
\text { DIS- } \\
\text { SOLVED } \\
\text { (MG/L } \\
\text { AS SO4) }\end{array}$ \\
\hline
\end{tabular}

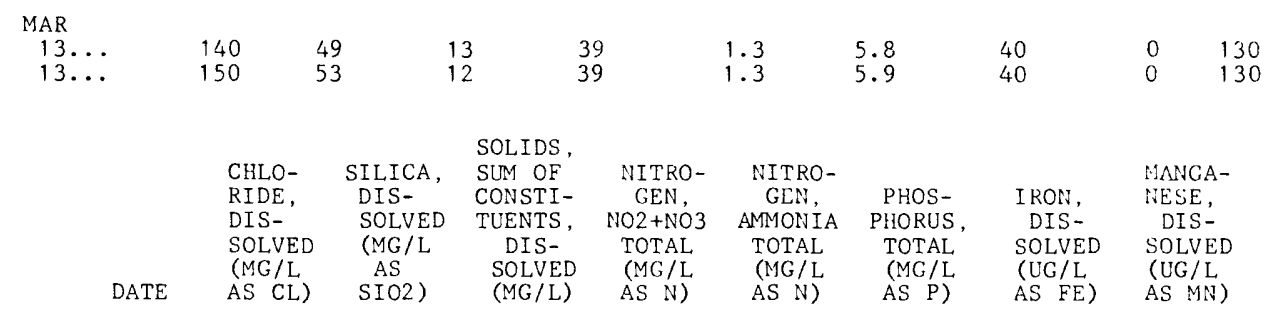

\begin{tabular}{|c|c|c|c|c|c|c|c|c|}
\hline MAR & 61 & & & & & $1 / 0$ & 20 & \\
\hline $13 \ldots$ & 66 & 9.2 & 335 & .05 & .080 & .150 & 20 & 130 \\
\hline
\end{tabular}


$\mathrm{FT}=$ feet $;$ MICROMHOS = micromhos per centimeter at $25^{\circ}$ Celsius; ${ }^{\circ} \mathrm{C}$ - degrees Celsius; $\mathrm{MG} / \mathrm{L}=$ milligrams per liter; UG/L = micrograms per liter

301908096313101 SOMERVILLE LAKE SITE AC

\begin{tabular}{|c|c|c|c|c|c|c|c|c|c|}
\hline DATE & TIME & $\begin{array}{l}\text { SAMP- } \\
\text { LING } \\
\text { DEPTH } \\
\text { (FT) }\end{array}$ & $\begin{array}{l}\text { SPE- } \\
\text { CIFIC } \\
\text { CON- } \\
\text { DUCT- } \\
\text { ANCE } \\
\text { (MICRO- } \\
\text { MHOS) }\end{array}$ & $\begin{array}{c}\text { PH } \\
\text { FIELD } \\
\text { (UNITS) }\end{array}$ & 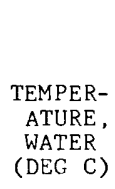 & $\begin{array}{l}\text { TRANS- } \\
\text { PAR- } \\
\text { ENCY } \\
\text { (SECCHI } \\
\text { DISK) } \\
\text { (M) }\end{array}$ & $\begin{array}{c}\text { OXYGEN, } \\
\text { DIS- } \\
\text { SOLVED } \\
\text { (MG/L) }\end{array}$ & $\begin{array}{c}\text { OXYGEN, } \\
\text { DIS- } \\
\text { SOLVED } \\
\text { (PER- } \\
\text { CENT } \\
\text { SATUR- } \\
\text { ATION) }\end{array}$ & $\begin{array}{l}\text { HARD- } \\
\text { NESS } \\
\text { (MG/L } \\
\text { AS } \\
\text { CACO3) }\end{array}$ \\
\hline $\begin{array}{l}\text { JUL } \\
14 \ldots \\
14 \ldots \\
14 \ldots \\
14 \ldots\end{array}$ & $\begin{array}{l}0922 \\
0924 \\
0927 \\
0930\end{array}$ & $\begin{array}{r}1.0 \\
10.0 \\
20.0 \\
27.0\end{array}$ & $\begin{array}{l}465 \\
465 \\
465 \\
467\end{array}$ & $\begin{array}{l}8.2 \\
7.5 \\
7.1 \\
7.1\end{array}$ & $\begin{array}{l}30.5 \\
30.0 \\
30.0 \\
30.0\end{array}$ & $\begin{array}{c}61 \\
-- \\
-- \\
--\end{array}$ & $\begin{array}{l}6.7 \\
5.1 \\
3.5 \\
3.3\end{array}$ & $\begin{array}{l}91 \\
69 \\
47 \\
45\end{array}$ & $\begin{array}{r}140 \\
-- \\
140\end{array}$ \\
\hline DATE & $\begin{array}{l}\text { HARD- } \\
\text { IIESS } \\
\text { NONCAR- } \\
\text { BOIATE } \\
\text { (NG/L } \\
\text { CACO3) }\end{array}$ & $\begin{array}{l}\text { CALCIUM } \\
\text { DIS- } \\
\text { SOLVED } \\
\text { (MG/L } \\
\text { AS CA) }\end{array}$ & $\begin{array}{l}\text { MAGNE- } \\
\text { SIUM, } \\
\text { DIS- } \\
\text { SOLVED } \\
\text { (MG/L } \\
\text { AS MG) }\end{array}$ & $\begin{array}{l}\text { SODIUM, } \\
\text { DIS- } \\
\text { SOLVED } \\
\text { (MG/L } \\
\text { AS NA) }\end{array}$ & $\begin{array}{c}\text { SODIUM } \\
\text { AD- } \\
\text { SORP- } \\
\text { TION } \\
\text { RATIO }\end{array}$ & $\begin{array}{l}\text { POTAS- } \\
\text { SIUM, } \\
\text { DIS- } \\
\text { SOLVED } \\
(M G / L \\
\text { AS K) }\end{array}$ & $\begin{array}{c}\text { BI CAR- } \\
\text { BONATE } \\
\text { (MG/L } \\
\text { AS } \\
\text { HCO3) }\end{array}$ & $\begin{array}{c}\text { CAR- } \\
\text { BONATE } \\
(\mathrm{MG} / \mathrm{L} \\
\text { AS CO3) }\end{array}$ & $\begin{array}{l}\text { SULFATE } \\
\text { DIS- } \\
\text { SOLVED } \\
\text { (MG/L } \\
\text { AS SO4) }\end{array}$ \\
\hline $\begin{array}{l}\text { JUL } \\
14 \ldots \\
14 \ldots \\
14 \ldots \\
14 \ldots\end{array}$ & $\begin{array}{l}85 \\
-- \\
-\overline{84}\end{array}$ & $39^{--}$ & $\begin{array}{c}9.4 \\
-- \\
-- \\
9.4\end{array}$ & 35 & $\begin{array}{l}1.3 \\
-- \\
1.2\end{array}$ & $\begin{array}{r}6.2 \\
-. \\
-- \\
6.2\end{array}$ & $\begin{array}{l}62 \\
-- \\
--\end{array}$ & $\begin{array}{r}0 \\
-- \\
-- \\
0\end{array}$ & $82^{--}$ \\
\hline $\mathrm{DA}$ & $\begin{array}{l}\text { CHLO- } \\
\text { RIDE, } \\
\text { DIS- } \\
\text { SOLVFD } \\
\text { (MC/L } \\
\text { AS CL) }\end{array}$ & $\begin{array}{l}\text { FLUO- } \\
\text { RIDE, } \\
\text { DIS- } \\
\text { SOLVED } \\
(M G / L \\
\text { AS F) }\end{array}$ & $\begin{array}{l}\text { SILICA, } \\
\text { DIS- } \\
\text { SOLVED } \\
\text { (MG/L } \\
\text { AS } \\
\text { SIO2) }\end{array}$ & $\begin{array}{l}\text { SOLIDS } \\
\text { SUM OF } \\
\text { CONSTI- } \\
\text { TUENTS, } \\
\text { DIS- } \\
\text { SOLVED } \\
\text { (MG/L) }\end{array}$ & $\begin{array}{c}\text { NITRO- } \\
\text { GEN, } \\
\text { NO2+NO3 } \\
\text { TOTAL } \\
\text { (MG/L } \\
\text { AS N) }\end{array}$ & $\begin{array}{l}\text { NITRO- } \\
\text { GEN, } \\
\text { AMMONIA } \\
\text { TOTAL } \\
\text { (MG/L } \\
\text { AS N) }\end{array}$ & $\begin{array}{l}\text { PHOS- } \\
\text { PHORUS, } \\
\text { TOTAL } \\
\text { (MG/L } \\
\text { AS P) }\end{array}$ & $\begin{array}{l}\text { IRON, } \\
\text { DIS- } \\
\text { SOLVED } \\
\text { (UG/L } \\
\text { AS FE) }\end{array}$ & $\begin{array}{l}\text { MANGA- } \\
\text { NESE, } \\
\text { DIS- } \\
\text { SOLVED } \\
\text { (UG/L } \\
\text { AS MN) }\end{array}$ \\
\hline
\end{tabular}

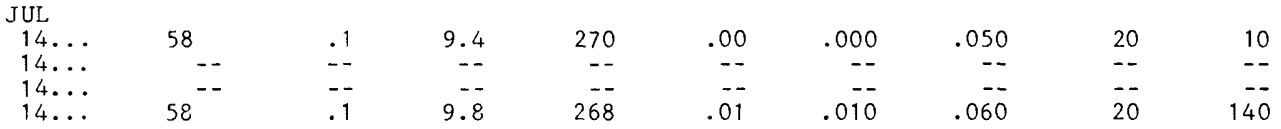

301940096315801 SOMERVILLE LAKE SITE AL

\begin{tabular}{|c|c|c|c|c|c|c|c|}
\hline DATE & TIME & $\begin{array}{l}\text { SAMP- } \\
\text { LING } \\
\text { DEPTH } \\
\text { (FT) }\end{array}$ & $\begin{array}{l}\text { SPE- } \\
\text { CIFIC } \\
\text { CON- } \\
\text { DUCT- } \\
\text { ANCE } \\
\text { (MICRO- } \\
\text { MHOS) }\end{array}$ & $\begin{array}{c}\text { PH } \\
\text { FIELD } \\
\text { (UNITS) }\end{array}$ & $\begin{array}{l}\text { TEMPER- } \\
\text { ATURE, } \\
\text { WATER } \\
\left(\begin{array}{ll}\text { DEG } & \text { C) }\end{array}\right.\end{array}$ & $\begin{array}{c}\text { OXYGEN } \\
\text { DIS- } \\
\text { SOLVED } \\
(\mathrm{MG} / \mathrm{L})\end{array}$ & $\begin{array}{l}\text { OXYGEN } \\
\text { DIS- } \\
\text { SOLVED } \\
\text { (PER- } \\
\text { CENT } \\
\text { SATUR- } \\
\text { ATION) }\end{array}$ \\
\hline IUL & & & & & & & \\
\hline $\begin{array}{l}14 \ldots \\
14 \ldots \\
14 \ldots\end{array}$ & $\begin{array}{l}0945 \\
0947 \\
0950\end{array}$ & $\begin{array}{r}1.0 \\
10.0 \\
20.0\end{array}$ & $\begin{array}{l}465 \\
465 \\
465\end{array}$ & $\begin{array}{l}8.5 \\
7.1 \\
7.0\end{array}$ & $\begin{array}{l}31.0 \\
30.0 \\
30.0\end{array}$ & $\begin{array}{l}7.8 \\
3.6 \\
2.6\end{array}$ & \\
\hline
\end{tabular}

302026096341501 SOMERVILLE LAKE SITE BC

\begin{tabular}{|c|c|c|c|c|c|c|c|}
\hline DATE & TIME & $\begin{array}{l}\text { SAMP- } \\
\text { LING } \\
\text { DEPTH } \\
(\mathrm{FT})\end{array}$ & $\begin{array}{l}\text { SPE- } \\
\text { CIFIC } \\
\text { CON- } \\
\text { DUCT- } \\
\text { ANCE } \\
\text { (MICRO- } \\
\text { MHOS) }\end{array}$ & $\begin{array}{c}\text { PH } \\
\text { FIELD } \\
\text { (UNITS) }\end{array}$ & $\begin{array}{c}\text { TEMPER- } \\
\text { ATURE, } \\
\text { WATER } \\
\text { (DEG C) }\end{array}$ & $\begin{array}{l}\text { TRANS - } \\
\text { PAR- } \\
\text { ENCY } \\
\text { (SECCHI } \\
\text { DISK) } \\
\text { (M) }\end{array}$ & $\begin{array}{c}\text { OXYGEN, } \\
\text { DIS- } \\
\text { SOLVED } \\
\text { (MG/L) }\end{array}$ \\
\hline \multicolumn{8}{|l|}{ JUL } \\
\hline $\begin{array}{l}14 \ldots \\
14 \ldots\end{array}$ & $\begin{array}{l}1000 \\
1003\end{array}$ & $\begin{array}{r}1.0 \\
10.0\end{array}$ & $\begin{array}{l}475 \\
475\end{array}$ & $\begin{array}{l}8.4 \\
8.4\end{array}$ & $\begin{array}{l}32.0 \\
31.5\end{array}$ & .55 & $\begin{array}{l}6.4 \\
6.3\end{array}$ \\
\hline
\end{tabular}


Table 10--CHEMICAL-QUALITY SURVEYS OF SOMERVILLE LAKE JULY 14, 1978--Continued

302026096341501 SOMERVILLE LAKE SITE BC--Continued

$\begin{array}{ccccccc} & \text { OXYGEN, } & & & & \\ & \text { DIS- } & \text { NITRO- } & \text { NITRO- } & & & \text { MANGA- } \\ & \text { SOLVED } & \text { GEN, } & \text { GEN, } & \text { PHOS- } & \text { IRON, } & \text { NESE, } \\ & \text { (PER- } & \text { NO2+NO3 } & \text { AMMONIA } & \text { PHORUS, } & \text { DIS- } & \text { DIS- } \\ & \text { CENT } & \text { TOTAL } & \text { TOTAL } & \text { TOTAL } & \text { SOLVED } & \text { SOLVED } \\ & \text { SATUR- } & \text { (MG/L } & \text { (MG/L } & \text { (MG /L } & \text { (UG/L } & \text { (UG /L } \\ \text { DATE } & \text { ATION) } & \text { AS N) } & \text { AS N) } & \text { AS P) } & \text { AS FE) } & \text { AS MN) }\end{array}$

$\begin{array}{lllllll}\text { JUL } & & & & & & \\ 14 \ldots & 89 & .01 & .000 & .040 & 20 & 0 \\ 14 \ldots & 86 & .01 & .000 & .040 & 30 & 0\end{array}$

301805096332501 SOMERVILLE LAKE SITE CC

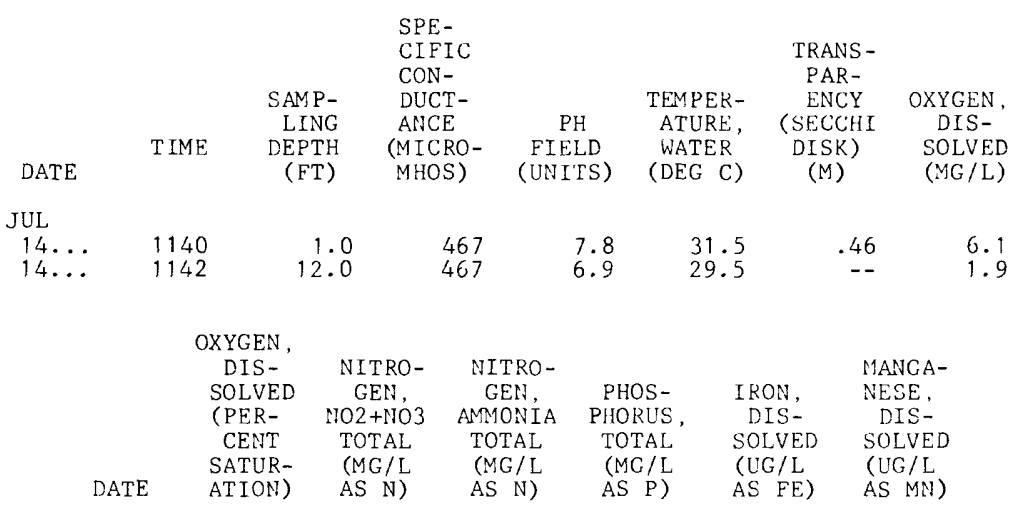

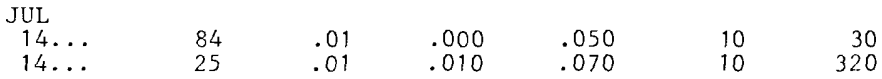

301847096334601 SOMERVILLE LAKE SITE DR

\begin{tabular}{|c|c|c|c|c|c|c|c|}
\hline DATE & TIME & $\begin{array}{l}\text { SAMP- } \\
\text { LING } \\
\text { DEPTH } \\
(\mathrm{FT})\end{array}$ & $\begin{array}{l}\text { SPE- } \\
\text { CIFIC } \\
\text { CON- } \\
\text { DUCT- } \\
\text { ANCE } \\
\text { (MICRO- } \\
\text { MHOS) }\end{array}$ & $\begin{array}{c}\text { PH } \\
\text { FIELD } \\
\text { (UNITS) }\end{array}$ & $\begin{array}{c}\text { TEMPER- } \\
\text { ATURE, } \\
\text { WATER } \\
\text { (DEG C) }\end{array}$ & $\begin{array}{c}\text { OXYGEN, } \\
\text { DIS- } \\
\text { SOLVED } \\
\text { (MG/L) }\end{array}$ & $\begin{array}{c}\text { OXYGEI, } \\
\text { DIS- } \\
\text { SOLVED } \\
\text { (PER- } \\
\text { CENT } \\
\text { SATUR- } \\
\text { ATION) }\end{array}$ \\
\hline JUL & & & & & & & \\
\hline $\begin{array}{l}14 \ldots \\
14 \ldots \\
14 \ldots\end{array}$ & $\begin{array}{l}1035 \\
1037 \\
1040\end{array}$ & $\begin{array}{r}1.0 \\
10.0 \\
20.0\end{array}$ & $\begin{array}{l}465 \\
465 \\
465\end{array}$ & $\begin{array}{l}8.4 \\
7.8 \\
7.1\end{array}$ & $\begin{array}{l}31.0 \\
30.5 \\
30.0\end{array}$ & $\begin{array}{l}6.8 \\
5.2 \\
2.9\end{array}$ & $\begin{array}{l}93 \\
70 \\
39\end{array}$ \\
\hline
\end{tabular}

301904096335601 SOMERVILLE LAKE SITE DC

\begin{tabular}{|c|c|c|c|c|c|c|c|c|c|}
\hline DATE & TIME & $\begin{array}{l}\text { SAMP- } \\
\text { LING } \\
\text { DEPTH } \\
\text { (FT) }\end{array}$ & $\begin{array}{l}\text { SPE- } \\
\text { CIFIC } \\
\text { CON- } \\
\text { DUCT- } \\
\text { ANCE } \\
\text { (MICRO- } \\
\text { MHOS) }\end{array}$ & $\begin{array}{c}\text { PH } \\
\text { FIELD } \\
\text { (UNITS) }\end{array}$ & $\begin{array}{l}\text { TEMPER- } \\
\text { ATURE, } \\
\text { WATER } \\
\text { (DEG C) }\end{array}$ & $\begin{array}{l}\text { TRANS- } \\
\text { PAR- } \\
\text { ENCY } \\
\text { (SECCHI } \\
\text { DISK) } \\
\text { (M) }\end{array}$ & $\begin{array}{c}\text { OXYGEN, } \\
\text { DIS- } \\
\text { SOLVED } \\
\text { (MG/L) }\end{array}$ & $\begin{array}{l}\text { OXYCEN, } \\
\text { DIS- } \\
\text { SOLVED } \\
\text { (PER- } \\
\text { CENT } \\
\text { SATUR- } \\
\text { ATION) }\end{array}$ & $\begin{array}{l}\text { HARD- } \\
\text { NESS } \\
(\mathrm{MC} / \mathrm{L} \\
\text { AS } \\
\text { CACO3) }\end{array}$ \\
\hline \multicolumn{10}{|l|}{ JUL } \\
\hline $\begin{array}{l}14 \ldots \\
14 \ldots \\
14 \ldots \\
14 \ldots\end{array}$ & $\begin{array}{l}1015 \\
1017 \\
1018 \\
1020\end{array}$ & $\begin{array}{r}1.0 \\
10.0 \\
20.0 \\
25.0\end{array}$ & $\begin{array}{l}465 \\
465 \\
465 \\
470\end{array}$ & $\begin{array}{l}8.5 \\
7.8 \\
7.0 \\
6.9\end{array}$ & $\begin{array}{l}31.5 \\
31.0 \\
30.0 \\
30.0\end{array}$ & $\begin{array}{c}67 \\
-- \\
-- \\
--\end{array}$ & $\begin{array}{r}7.0 \\
4.9 \\
2.1 \\
.3\end{array}$ & $\begin{array}{r}96 \\
67 \\
28 \\
4\end{array}$ & $\begin{array}{r}140 \\
-- \\
140\end{array}$ \\
\hline
\end{tabular}


Table 10--CHEMICAL-QUALITY SURVEYS OF SOMERVILLE LAKE JULY 14, 1978--Continued

\begin{tabular}{|c|c|c|c|c|c|c|c|c|c|}
\hline & & 3019040 & 6335601 & SOMERVI & LAKE & TE DC--C & ontinued & & \\
\hline DATE & $\begin{array}{l}\text { HARD- } \\
\text { NESS, } \\
\text { NONCAR- } \\
\text { BONATE } \\
\text { (MG/L } \\
\text { CACO3) }\end{array}$ & $\begin{array}{l}\text { CALCIUM } \\
\text { DIS- } \\
\text { SOLVED } \\
\text { (MG/L } \\
\text { AS CA) }\end{array}$ & $\begin{array}{l}\text { MAGNE- } \\
\text { SIUM, } \\
\text { DIS- } \\
\text { SOLVED } \\
\text { (MC/L } \\
\text { AS MG) }\end{array}$ & $\begin{array}{l}\text { SODIUM, } \\
\text { DIS- } \\
\text { SOLVED } \\
\text { (MG/L } \\
\text { AS NA) }\end{array}$ & $\begin{array}{c}\text { SODIUM } \\
\text { AD- } \\
\text { SORP- } \\
\text { TION } \\
\text { RATIO }\end{array}$ & $\begin{array}{l}\text { POTAS- } \\
\text { SIUM, } \\
\text { DIS- } \\
\text { SOLVED } \\
\text { (MG/L } \\
\text { AS K) }\end{array}$ & $\begin{array}{c}\text { BICAR- } \\
\text { BONATE } \\
\text { (MG /L } \\
\text { AS } \\
\text { HCO3) }\end{array}$ & $\begin{array}{c}\text { CAR- } \\
\text { BONATE } \\
\text { (MG / L } \\
\text { AS CO3) }\end{array}$ & $\begin{array}{l}\text { SULFATE } \\
\text { DIS- } \\
\text { SOLVED } \\
\text { (MG/L } \\
\text { AS SO4) }\end{array}$ \\
\hline
\end{tabular}

\begin{tabular}{|c|c|c|c|c|c|c|c|c|c|}
\hline JUL & & & & & & & & & \\
\hline $14 \ldots$ & 86 & 39 & 9.5 & 33 & 1.2 & 6.2 & 54 & 4 & 83 \\
\hline $14 \ldots$ & -- & -- & -- & -- & -- & -- & $\rightarrow$ & -- & \\
\hline $14 \ldots$ & $\overline{-1}$ & $0^{--}$ & -- & - & -- & -- & $=$ & - & \\
\hline $14 \ldots$ & 83 & 40 & 9.4 & 34 & 1.3 & 6.2 & 68 & 0 & 80 \\
\hline
\end{tabular}

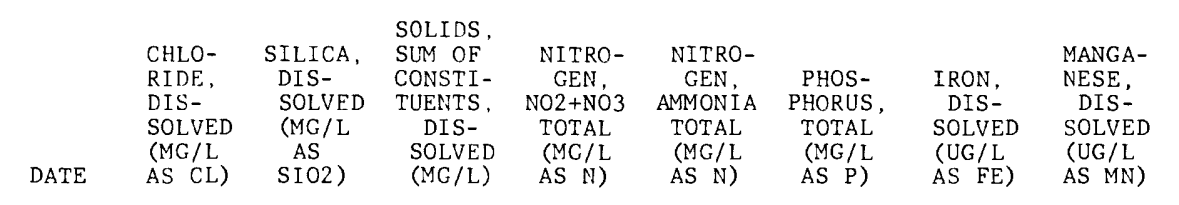

\begin{tabular}{|c|c|c|c|c|c|c|c|c|}
\hline JUL & & & & & & & & \\
\hline $14 \ldots$ & 56 & 9.6 & 267 & .00 & .000 & .050 & 30 & 10 \\
\hline $14 \ldots$ & -- & -- & -- & -- & -- & -- & -- & -- \\
\hline $14 \ldots$ & -- & -- & - & -- & -- & -- & -- & -- \\
\hline $14 \ldots$ & 59 & 10 & 273 & .01 & .080 & .090 & 110 & 660 \\
\hline
\end{tabular}

301817096364101 SOMERVILLE LAKE SITE EC

\begin{tabular}{|c|c|c|c|c|c|c|c|}
\hline DATE & TIME & $\begin{array}{c}\text { SAMP- } \\
\text { LING } \\
\text { DEPTH } \\
(\mathrm{FT})\end{array}$ & $\begin{array}{l}\text { SPE- } \\
\text { CIFIC } \\
\text { CON- } \\
\text { DUCT- } \\
\text { ANCE } \\
\text { (MICRO- } \\
\text { MHOS) }\end{array}$ & $\begin{array}{c}\text { PH } \\
\text { FIELD } \\
\text { (UNITS) }\end{array}$ & $\begin{array}{c}\text { TEMPER- } \\
\text { ATURE, } \\
\text { WATER } \\
\text { (DEG C) }\end{array}$ & $\begin{array}{c}\text { OXYGEN, } \\
\text { DIS- } \\
\text { SOLVED } \\
\text { (MG } / \mathrm{L})\end{array}$ & $\begin{array}{c}\text { OXYGEN, } \\
\text { DIS- } \\
\text { SOLVED } \\
\text { (PER- } \\
\text { CENT } \\
\text { SATUR- } \\
\text { ATION) }\end{array}$ \\
\hline \multicolumn{8}{|l|}{ JUL } \\
\hline $\begin{array}{l}14 \ldots \\
14 \ldots \\
14 \ldots\end{array}$ & $\begin{array}{l}1050 \\
1052 \\
1055\end{array}$ & $\begin{array}{r}1.0 \\
10.0 \\
21.0\end{array}$ & $\begin{array}{l}465 \\
465 \\
465\end{array}$ & $\begin{array}{l}7.9 \\
7.8 \\
7.3\end{array}$ & $\begin{array}{l}30.5 \\
30.0 \\
30.0\end{array}$ & $\begin{array}{l}6.0 \\
5.7 \\
4.2\end{array}$ & $\begin{array}{l}81 \\
77 \\
57\end{array}$ \\
\hline
\end{tabular}

301754096380801 SOMERVILLE LAKE SITE FC

\begin{tabular}{|c|c|c|c|c|c|c|c|c|c|}
\hline & & $\begin{array}{l}\text { SAMP- } \\
\text { LING }\end{array}$ & $\begin{array}{l}\text { SPE- } \\
\text { CIFIC } \\
\text { CON- } \\
\text { DUCT- } \\
\text { ANCE }\end{array}$ & $\mathrm{PH}$ & $\begin{array}{l}\text { TEMPER- } \\
\text { ATURE, }\end{array}$ & $\begin{array}{c}\text { TRANS- } \\
\text { PAR- } \\
\text { ENCY } \\
\text { (SECCHI }\end{array}$ & $\begin{array}{c}\text { OXYGEN, } \\
\text { DIS- }\end{array}$ & $\begin{array}{c}\text { OXYGEN } \\
\text { DIS- } \\
\text { SOLVED } \\
\text { (PER- } \\
\text { CENT }\end{array}$ & $\begin{array}{l}\text { HARD- } \\
\text { NESS } \\
\text { (MG/L }\end{array}$ \\
\hline DATE & TIME & $\begin{array}{c}\text { DEPTH } \\
\text { (FT) }\end{array}$ & $\begin{array}{l}\text { (MICRO- } \\
\text { MHOS) }\end{array}$ & $\begin{array}{l}\text { FIELD } \\
\text { (UNITS) }\end{array}$ & $\begin{array}{c}\text { WATER } \\
\text { (DEG } \quad \text { C) }\end{array}$ & $\begin{array}{c}\text { DISK) } \\
(\mathrm{M})\end{array}$ & $\begin{array}{l}\text { SOLVED } \\
(\mathrm{MG} / \mathrm{L})\end{array}$ & $\begin{array}{l}\text { SATUR- } \\
\text { ATION) }\end{array}$ & $\begin{array}{c}\text { AS } \\
\text { (ACO3) }\end{array}$ \\
\hline $\begin{array}{l}\text { JUL } \\
14 \ldots \\
14 \ldots\end{array}$ & $\begin{array}{l}1111 \\
1114\end{array}$ & $\begin{array}{l}1.0 \\
8.0\end{array}$ & $\begin{array}{l}478 \\
480\end{array}$ & $\begin{array}{l}7.3 \\
7.1\end{array}$ & $\begin{array}{l}31.0 \\
31.0\end{array}$ & .24 & $\begin{array}{l}4.8 \\
2.8\end{array}$ & $\begin{array}{l}66 \\
38\end{array}$ & $\begin{array}{l}140 \\
140\end{array}$ \\
\hline DATE & $\begin{array}{l}\text { HARD- } \\
\text { NESS, } \\
\text { NONCAR- } \\
\text { BONATE } \\
\text { (MG/L } \\
\text { CACO3) }\end{array}$ & $\begin{array}{l}\text { CALCI UM } \\
\text { DIS- } \\
\text { SOLVED } \\
\text { (MG/L } \\
\text { AS CA) }\end{array}$ & $\begin{array}{l}\text { MAGNE- } \\
\text { SIUM, } \\
\text { DIS- } \\
\text { SOLVED } \\
\text { (MG/L } \\
\text { AS MG) }\end{array}$ & $\begin{array}{l}\text { SODIUM, } \\
\text { DIS- } \\
\text { SOLVED } \\
\text { (MG/L } \\
\text { AS NA) }\end{array}$ & $\begin{array}{c}\text { SODIUM } \\
\text { AD- } \\
\text { SORP- } \\
\text { TION } \\
\text { RATIO }\end{array}$ & $\begin{array}{l}\text { POTAS- } \\
\text { SIUM, } \\
\text { DIS- } \\
\text { SOLVED } \\
(\text { MG/L } \\
\text { AS K) }\end{array}$ & $\begin{array}{c}\text { BICAR- } \\
\text { BONATE } \\
\text { (MG /L } \\
\text { AS } \\
\text { HCO3) }\end{array}$ & $\begin{array}{c}\text { CAR- } \\
\text { BONATE } \\
\text { (MG/L } \\
\text { AS CO3) }\end{array}$ & $\begin{array}{l}\text { SULFATE } \\
\text { DIS- } \\
\text { SOLVED } \\
\text { (MG/L } \\
\text { AS SO4) }\end{array}$ \\
\hline
\end{tabular}

\begin{tabular}{|c|c|c|c|c|c|c|c|c|}
\hline \multirow[t]{2}{*}{$\begin{array}{l}\text { JUL } \\
14 \ldots \\
14 \ldots\end{array}$} & $\begin{array}{l}87 \\
91\end{array}$ & & $\begin{array}{l}9.7 \\
9.9\end{array}$ & & $\begin{array}{l}1.3 \\
1.2\end{array}$ & $\begin{array}{l}6.8 \\
6.5\end{array}$ & $\begin{array}{l}64 \\
64\end{array}$ & $\begin{array}{l}0 \\
0\end{array}$ \\
\hline & $\begin{array}{l}\text { CHLO- } \\
\text { RIDE, } \\
\text { DIS- } \\
\text { SOLVED } \\
\text { (MG/L } \\
\text { AS CL) }\end{array}$ & $\begin{array}{l}\text { SILICA, } \\
\text { DIS- } \\
\text { SOLVED } \\
\text { (MG/L } \\
\text { AS } \\
\text { SIO2) }\end{array}$ & $\begin{array}{c}\text { SOLIDS, } \\
\text { SUM OF } \\
\text { CONSTI- } \\
\text { TUENTS, } \\
\text { DIS- } \\
\text { SOLVED } \\
\text { (MG/L) }\end{array}$ & $\begin{array}{c}\text { NITRO- } \\
\text { GEN, } \\
\text { NO2+NO3 } \\
\text { TOTAL } \\
\text { (MG/L } \\
\text { AS N) }\end{array}$ & $\begin{array}{l}\text { NITRO- } \\
\text { GEN } \\
\text { AMMONIA } \\
\text { TOTAL } \\
\text { (MG/L } \\
\text { AS N) }\end{array}$ & $\begin{array}{c}\text { PHOS- } \\
\text { PHORUS, } \\
\text { TOTAL } \\
\text { (MG/L } \\
\text { AS P) }\end{array}$ & $\begin{array}{l}\text { IRON, } \\
\text { DIS- } \\
\text { SOLVED } \\
\text { (UG/L } \\
\text { AS FE) }\end{array}$ & $\begin{array}{l}\text { MANGA- } \\
\text { NESE, } \\
\text { DIS- } \\
\text { SOLVED } \\
\text { (UG / L } \\
\text { AS MN) }\end{array}$ \\
\hline
\end{tabular}

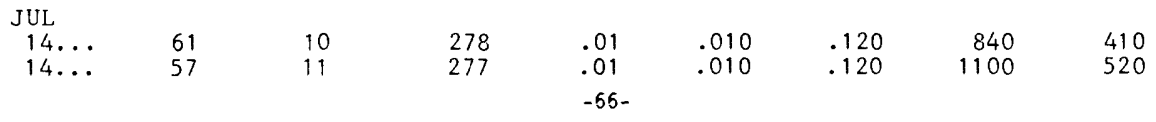


$\mathrm{FT}=$ feet $;$ MICROMHOS = micromhos per centimeter at $25^{\circ}$ Celsius; ${ }^{\circ} \mathrm{C}-$ degrees Celsius; $\mathrm{MG} / \mathrm{L}=$ milligrams per liter; $\mathrm{UC} / \mathrm{L}=$ micrograms per liter

301908096313101 SOMERVILLE LAKE SITE AC

\begin{tabular}{|c|c|c|c|c|c|c|c|c|c|}
\hline DATE & TIME & $\begin{array}{l}\text { SAMP- } \\
\text { LING } \\
\text { DEPTH } \\
(\mathrm{FT})\end{array}$ & $\begin{array}{l}\text { SPE- } \\
\text { CIFIC } \\
\text { CON- } \\
\text { DUCT- } \\
\text { ANCE } \\
\text { (MICRO- } \\
\text { MHOS) }\end{array}$ & $\begin{array}{c}\text { PH } \\
\text { FIELD } \\
\text { (UNITS) }\end{array}$ & $\begin{array}{l}\text { TEMPER- } \\
\text { ATURE, } \\
\text { WATER } \\
\text { (DEG C) }\end{array}$ & $\begin{array}{l}\text { TRANS- } \\
\text { PAR - } \\
\text { ENCY } \\
\text { (SECCHI } \\
\text { DISK) } \\
\text { (M) }\end{array}$ & $\begin{array}{c}\text { OXYGEN, } \\
\text { DIS- } \\
\text { SOLVED } \\
\text { (MG/L) }\end{array}$ & $\begin{array}{c}\text { OXYGEN, } \\
\text { DIS- } \\
\text { SOLVED } \\
\text { (PER- } \\
\text { CENT } \\
\text { SATUR- } \\
\text { ATION) }\end{array}$ & $\begin{array}{l}\text { HARD- } \\
\text { NESS } \\
(\mathrm{MG} / \mathrm{L} \\
\text { AS } \\
\mathrm{CACO} 3)\end{array}$ \\
\hline $\begin{array}{l}\text { OCT } \\
11 \ldots \\
11 \ldots \\
11 \ldots\end{array}$ & $\begin{array}{l}1115 \\
1120 \\
1125\end{array}$ & $\begin{array}{r}1.0 \\
10.0 \\
23.0\end{array}$ & $\begin{array}{l}479 \\
479 \\
479\end{array}$ & $\begin{array}{l}7.8 \\
7.7 \\
7.4\end{array}$ & $\begin{array}{l}24.0 \\
23.5 \\
23.5\end{array}$ & $\begin{array}{l}.70 \\
-- \\
--\end{array}$ & $\begin{array}{l}7.6 \\
7.3 \\
6.5\end{array}$ & $\begin{array}{l}93 \\
89 \\
78\end{array}$ & $\begin{array}{r}140 \\
140\end{array}$ \\
\hline DATE & $\begin{array}{l}\text { HARD- } \\
\text { NESS, } \\
\text { NONCAR- } \\
\text { BONATE } \\
\text { (MG/L } \\
\text { CACO3) }\end{array}$ & $\begin{array}{l}\text { CALCIUM } \\
\text { DIS- } \\
\text { SOLVED } \\
\text { (MG/L } \\
\text { AS CA) }\end{array}$ & $\begin{array}{l}\text { MAGNE- } \\
\text { SIUM, } \\
\text { DIS- } \\
\text { SOLVED } \\
\text { (MG/L } \\
\text { AS MG) }\end{array}$ & $\begin{array}{l}\text { SODIUM, } \\
\text { DIS- } \\
\text { SOLVED } \\
\text { (MG/L } \\
\text { AS NA) }\end{array}$ & $\begin{array}{c}\text { SODIUM } \\
\text { AD- } \\
\text { SORP- } \\
\text { TION } \\
\text { RATIO }\end{array}$ & $\begin{array}{l}\text { POTAS- } \\
\text { SIUM, } \\
\text { DIS- } \\
\text { SOLVED } \\
\text { (MG/L } \\
\text { AS K) }\end{array}$ & $\begin{array}{c}\text { BICAR- } \\
\text { BONATE } \\
\text { (MG /L } \\
\text { AS } \\
\text { HCO3) }\end{array}$ & $\begin{array}{c}\text { CAR- } \\
\text { BONATE } \\
\text { (MG/L } \\
\text { AS CO3) }\end{array}$ & $\begin{array}{l}\text { SULFATE } \\
\text { DIS- } \\
\text { SOLVED } \\
\text { (MG/L } \\
\text { AS SO4) }\end{array}$ \\
\hline $\begin{array}{r}\text { OCT } \\
11 \ldots \\
11 \ldots \\
11 \ldots\end{array}$ & $\begin{array}{l}82 \\
-- \\
81\end{array}$ & $40^{--}$ & $\begin{array}{r}8.8 \\
-\overline{6} \\
8.6\end{array}$ & 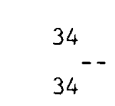 & $\begin{array}{r}1.3 \\
-2 \\
1.3\end{array}$ & $\begin{array}{r}6.6 \\
-.6 \\
6.6\end{array}$ & $\begin{array}{l}66 \\
-- \\
66\end{array}$ & $\begin{array}{r}0 \\
- \\
-\end{array}$ & ${ }_{87^{90}}^{--}$ \\
\hline DATE & $\begin{array}{l}\text { CHLO- } \\
\text { RIDE, } \\
\text { DIS- } \\
\text { SOLVED } \\
\text { (MG/L } \\
\text { AS CL) }\end{array}$ & $\begin{array}{l}\text { FLUO- } \\
\text { RIDE, } \\
\text { DIS- } \\
\text { SOLVED } \\
\text { (MG/L } \\
\text { AS F) }\end{array}$ & $\begin{array}{l}\text { SILICA, } \\
\text { DIS- } \\
\text { SOLVED } \\
\text { (MG/L } \\
\text { AS } \\
\text { SIO2) }\end{array}$ & $\begin{array}{l}\text { SOLIDS, } \\
\text { SUM OF } \\
\text { CONSTI- } \\
\text { TUENTS, } \\
\text { DIS- } \\
\text { SOLVED } \\
\text { (MC/L) }\end{array}$ & $\begin{array}{c}\text { NITRO- } \\
\text { GEN } \\
\text { NO2+NO3 } \\
\text { TOTAL } \\
\text { (MG/L } \\
\text { AS N) }\end{array}$ & $\begin{array}{c}\text { NITRO- } \\
\text { GEN, } \\
\text { AMMONIA } \\
\text { TOTAL } \\
\text { (MG/L } \\
\text { AS N) }\end{array}$ & $\begin{array}{l}\text { PHOS- } \\
\text { PHORUS, } \\
\text { TOTAL } \\
\text { (MG/L } \\
\text { AS P) }\end{array}$ & $\begin{array}{l}\text { IRON, } \\
\text { DIS- } \\
\text { SOLVED } \\
\text { (UG/L } \\
\text { AS FE) }\end{array}$ & $\begin{array}{l}\text { MANGA- } \\
\text { NESE, } \\
\text { DIS- } \\
\text { SOLVED } \\
\text { (UG/L } \\
\text { AS MN) }\end{array}$ \\
\hline $\begin{array}{c}\text { OCT } \\
11 \ldots \\
11 \ldots \\
11 \ldots\end{array}$ & $\begin{array}{l}57 \\
57^{--}\end{array}$ & $\begin{array}{l}.2 \\
-. \\
--\end{array}$ & $\begin{array}{l}11 \\
11^{-}\end{array}$ & $\begin{array}{r}280 \\
-\overline{277}\end{array}$ & $\begin{array}{l}.00 \\
.01 \\
.00\end{array}$ & $\begin{array}{l}.010 \\
.010 \\
.010\end{array}$ & $\begin{array}{l}.060 \\
.060 \\
.070\end{array}$ & $\begin{array}{r}10 \\
<10 \\
20\end{array}$ & $\begin{array}{r}0 \\
1 \\
40\end{array}$ \\
\hline
\end{tabular}

301940096315801 SOMERVILLE LAKE SITE AL

\begin{tabular}{|c|c|c|c|c|c|c|c|c|}
\hline DATE & TIME & $\begin{array}{c}\text { SAMP- } \\
\text { LING } \\
\text { DEPTH } \\
\text { (FT) }\end{array}$ & $\begin{array}{l}\text { SPE- } \\
\text { CIFIC } \\
\text { CON- } \\
\text { DUCT- } \\
\text { ANCE } \\
\text { (MICRO- } \\
\text { MHOS) }\end{array}$ & $\begin{array}{c}\text { PH } \\
\text { FIELD } \\
\text { (UNITS) }\end{array}$ & $\begin{array}{l}\text { TEMPER- } \\
\text { ATURE, } \\
\text { WATER } \\
\text { (DEG C) }\end{array}$ & $\begin{array}{l}\text { TRANS- } \\
\text { PAR- } \\
\text { ENCY } \\
\text { (SECCHI } \\
\text { DISK) } \\
\text { (M) }\end{array}$ & $\begin{array}{c}\text { OXYGEN, } \\
\text { DIS- } \\
\text { SOLVED } \\
\text { (MG/L) }\end{array}$ & $\begin{array}{c}\text { OXYGEN, } \\
\text { DIS- } \\
\text { SOLVED } \\
\text { (PER- } \\
\text { CENT } \\
\text { SATUR- } \\
\text { ATION) }\end{array}$ \\
\hline OCT & & & & & & & & \\
\hline $\begin{array}{l}11 \ldots \\
11 \ldots \\
11 \ldots\end{array}$ & $\begin{array}{l}1126 \\
1128 \\
1130\end{array}$ & $\begin{array}{r}1.0 \\
10.0 \\
23.0\end{array}$ & $\begin{array}{l}479 \\
479 \\
479\end{array}$ & $\begin{array}{l}7.7 \\
7.7 \\
7.7\end{array}$ & $\begin{array}{l}24.0 \\
23.5 \\
23.5\end{array}$ & $\begin{array}{c}.40 \\
-- \\
--\end{array}$ & $\begin{array}{l}7.2 \\
7.2 \\
7.2\end{array}$ & $\begin{array}{l}89 \\
87 \\
87\end{array}$ \\
\hline
\end{tabular}

302026096341501 SOMERVILLE LAKE SITE BC

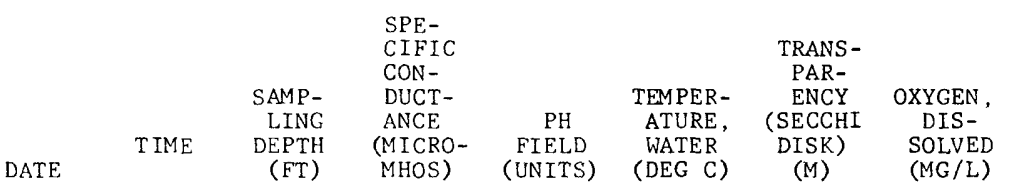

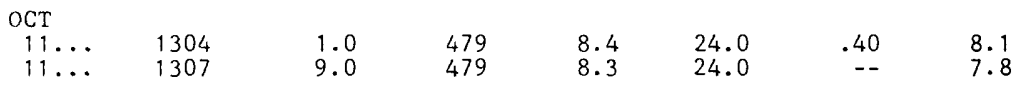


Table 11--CHEMICAL-QUALITY SURVEYS OF SOMERVILLE LAKE OCTOBER 11, 1978--Continued

302026096341501 SOMERVILLE LAKE SITE BC--Continued

\begin{tabular}{ccccccc}
\multicolumn{7}{c}{ OXYGEN, } \\
DIS- & NITRO- & NITRO- & & & \\
& SOLVED & GEN, & GEN, & PHOS- & IRON, & MANGA- \\
& (PER- & NO2+NO3 & AMMONIA & PHORUS, & DIS- & DIS- \\
& CENT & TOTAL & TOTAL & TOTAL & SOLVED & SOLVED \\
& SATUR- & (MG/L & (MG/L & (MG/L & (UG/L & (UC/L \\
DATE & ATION) & AS N) & AS N) & AS P) & AS FE) & AS MN)
\end{tabular}

\begin{tabular}{|c|c|c|c|c|c|}
\hline $\begin{array}{l}\text { OCT } \\
11 \ldots\end{array}$ & 100 & .00 & .000 & .070 & $<10$ \\
\hline $11 \ldots$ & 96 & .00 & .010 & .070 & $<10$ \\
\hline
\end{tabular}

301805096332501 SOMERVILLE LAKE SITE CC

\begin{tabular}{|c|c|c|c|c|c|c|c|}
\hline DATE & TIME & $\begin{array}{l}\text { SAMP- } \\
\text { LING } \\
\text { DEPTH } \\
\text { (FT) }\end{array}$ & $\begin{array}{l}\text { SPE- } \\
\text { CIFIC } \\
\text { CON- } \\
\text { DUCT- } \\
\text { ANCE } \\
\text { (MICRO- } \\
\text { MHOS) }\end{array}$ & $\begin{array}{c}\text { PH } \\
\text { FIELD } \\
\text { (UNITS) }\end{array}$ & $\begin{array}{c}\text { TEMPER- } \\
\text { ATURE, } \\
\text { WATER } \\
\text { (DEG } \\
\text { (D) }\end{array}$ & $\begin{array}{c}\text { TRANS- } \\
\text { PAR- } \\
\text { ENCY } \\
\text { (SECCHI } \\
\text { DISK) } \\
(M)\end{array}$ & $\begin{array}{c}\text { OXYGEN, } \\
\text { DIS - } \\
\text { SOLVED } \\
(M G / L)\end{array}$ \\
\hline $\mathrm{CT}$ & & & & & & & \\
\hline $\begin{array}{l}11 \ldots \\
11 \ldots\end{array}$ & $\begin{array}{l}1232 \\
1235\end{array}$ & $\begin{array}{r}1.0 \\
10.0\end{array}$ & $\begin{array}{l}479 \\
479\end{array}$ & $\begin{array}{l}7.8 \\
7.7\end{array}$ & $\begin{array}{l}23.5 \\
23.5\end{array}$ & $\begin{array}{c}.60 \\
--\end{array}$ & $\begin{array}{l}7.2 \\
7.0\end{array}$ \\
\hline
\end{tabular}

\begin{tabular}{ccccccc}
\multicolumn{7}{c}{ OXYGEN, } \\
DIS- & NITRO- & NITRO- & & & \\
& SOLVED & GEN, & GEN, & PHOS- & IRON, & NANGA- \\
& (PER- & NO2+NO3 & AMMONIA & PHORUS, & DIS- & DIS- \\
& CENT & TOTAL & TOTAL & TOTAL & SOLVED & SOLVED \\
& SATUR- & (MG/L & (MG/L & (MG/L & (UG/L & (UG/L \\
DATE & ATION) & AS N) & AS N) & AS P) & AS FE) & AS MN)
\end{tabular}

\begin{tabular}{|c|c|c|c|c|c|}
\hline $\mathrm{OCT}$ & & & & & \\
\hline $\begin{array}{l}11 \ldots \\
11 \ldots\end{array}$ & $\begin{array}{l}88 \\
84\end{array}$ & $\begin{array}{l}.01 \\
.00\end{array}$ & $\begin{array}{l}.010 \\
.010\end{array}$ & $\begin{array}{l}.070 \\
.070\end{array}$ & $\begin{array}{l}<10 \\
<10\end{array}$ \\
\hline
\end{tabular}

301847096334601 SOMERVILLE LAKE SITE DR

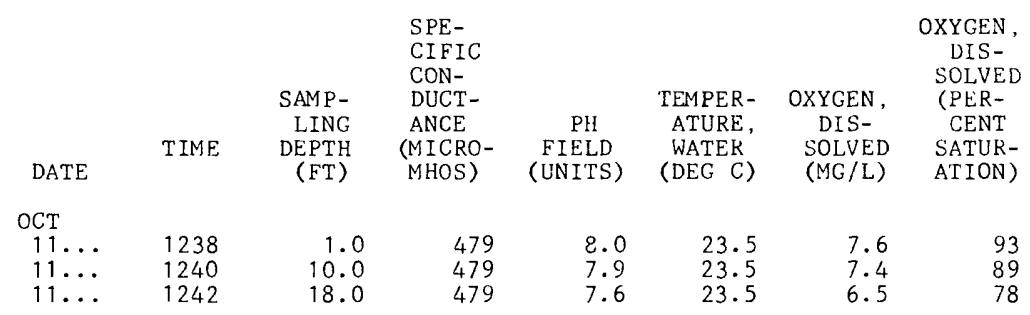

301904096335601 SOMERVILLE LAKE SITE DC

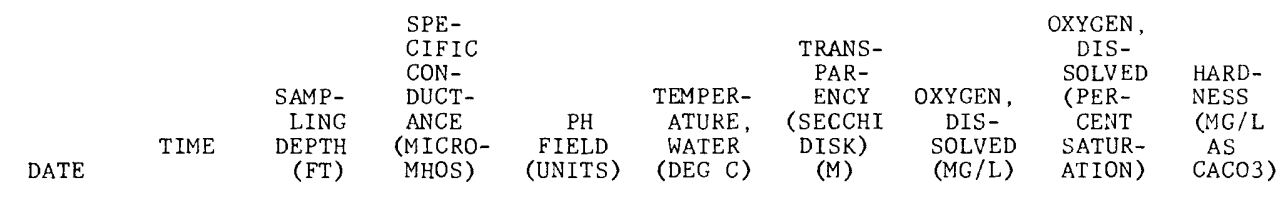

\begin{tabular}{|c|c|c|c|c|c|c|c|c|c|}
\hline OCT & & & & & & & & & \\
\hline $11 \ldots$ & 1248 & 1.0 & 479 & 8.0 & 24.0 & .60 & 7.5 & 93 & 140 \\
\hline $11 \ldots$ & 1251 & 10.0 & 479 & 7.9 & 24.0 & - & 7.3 & 89 & \\
\hline $11 \ldots$ & 1254 & 20.0 & 479 & 7.6 & 23.5 & -- & 6.6 & 81 & 14 \\
\hline
\end{tabular}


Table 11--CHEMICAL-QUALITY SURVEYS OF SOMERVILLE LAKE OCTOBER 11, 1978--Continued

301904096335601 SOMERVILLE LAKE SITE DC--Continued

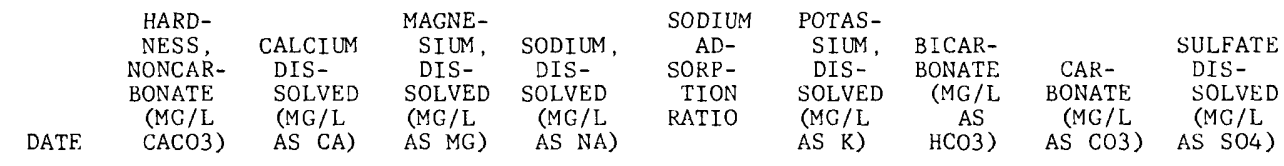

\begin{tabular}{|c|c|c|c|c|c|c|c|c|c|}
\hline OCT & & & & & & & & & \\
\hline $11 \ldots$ & 83 & 40 & 8.6 & 34 & 1.3 & 6.5 & 64 & 0 & 89 \\
\hline $11 \ldots$ & -- & -- & -- & -- & -- & $\cdots$ & -- & -- & \\
\hline $11 \ldots$ & 82 & 40 & 8.7 & 34 & 1.3 & 6.5 & 65 & 0 & 85 \\
\hline
\end{tabular}

\begin{tabular}{|c|c|c|c|c|c|c|c|c|}
\hline & $\begin{array}{l}\text { CHLO- } \\
\text { RIDE, } \\
\text { DIS- } \\
\text { SOLVED } \\
\text { (MG / L }\end{array}$ & $\begin{array}{l}\text { SILICA, } \\
\text { DIS- } \\
\text { SOLVED } \\
\text { (MG/L } \\
\text { AS }\end{array}$ & $\begin{array}{l}\text { SOLIDS, } \\
\text { SUM OF } \\
\text { CONSTI- } \\
\text { TUENTS, } \\
\text { DIS- } \\
\text { SOLVED }\end{array}$ & $\begin{array}{l}\text { NITRO- } \\
\text { GEN, } \\
\text { NO2+NO3 } \\
\text { TOTAL } \\
\text { (MG/L }\end{array}$ & $\begin{array}{c}\text { NITRO- } \\
\text { GEN } \\
\text { AMMONIA } \\
\text { TOTAL } \\
\text { (MG/L }\end{array}$ & $\begin{array}{c}\text { PHOS- } \\
\text { PHORUS, } \\
\text { TOTAL } \\
\text { (MG/L }\end{array}$ & $\begin{array}{l}\text { IRON, } \\
\text { DIS- } \\
\text { SOLVED } \\
\text { (UC/L }\end{array}$ & $\begin{array}{l}\text { MANGA- } \\
\text { NESE, } \\
\text { DIS- } \\
\text { SOLVED } \\
\text { (UG/L }\end{array}$ \\
\hline ATE & AS CL) & SIO2) & $(\mathrm{MC} / \mathrm{L})$ & AS N) & AS N) & AS P) & AS FE) & AS MN) \\
\hline
\end{tabular}

\begin{tabular}{|c|c|c|c|c|c|c|c|c|}
\hline $\mathrm{OCT}$ & & & & & & & & \\
\hline $11 \ldots$ & 56 & 11 & 277 & .01 & .010 & .070 & 20 & 0 \\
\hline $11 \ldots$ & $56^{--}$ & $11^{--}$ & $2 \overline{3}$ & $\overline{0}$ & $0 \overline{0}$ & -- & $\overline{10}$ & 0 \\
\hline
\end{tabular}

301817096364101 SOMERVILLF, LAKE SITE EC

\begin{tabular}{|c|c|c|c|c|c|c|c|}
\hline & & & $\begin{array}{l}\text { SPE- } \\
\text { CIFIC } \\
\text { CON- }\end{array}$ & & & & $\begin{array}{c}\text { OXYGEN, } \\
\text { DIS- } \\
\text { SOLVED }\end{array}$ \\
\hline DATE & T IME & $\begin{array}{l}\text { SAMP- } \\
\text { LING } \\
\text { DEPTH } \\
(F T)\end{array}$ & $\begin{array}{l}\text { DUCT- } \\
\text { ANCE } \\
\text { (MICRO- } \\
\text { MHOS) }\end{array}$ & $\begin{array}{c}\text { PH } \\
\text { FIELD } \\
\text { (UNITS) }\end{array}$ & $\begin{array}{l}\text { TEMPER- } \\
\text { ATURE, } \\
\text { WATER } \\
\text { (DEG C) }\end{array}$ & $\begin{array}{c}\text { OXYGEN, } \\
\text { DIS- } \\
\text { SOLVED } \\
\text { (MG/L) }\end{array}$ & $\begin{array}{l}\text { (PER- } \\
\text { CENT } \\
\text { SATUR- } \\
\text { ATION) }\end{array}$ \\
\hline
\end{tabular}

\begin{tabular}{|c|c|c|c|c|c|c|c|}
\hline \multicolumn{8}{|l|}{ OCT } \\
\hline $11 \ldots$ & $\begin{array}{l}1212 \\
1214 \\
1216\end{array}$ & $\begin{array}{r}1.0 \\
10.0 \\
10\end{array}$ & $\begin{array}{l}479 \\
479 \\
479\end{array}$ & $\begin{array}{l}7.8 \\
7.7 \\
7.6\end{array}$ & $\begin{array}{l}24.0 \\
23.5 \\
23\end{array}$ & $\begin{array}{l}7.2 \\
6.9 \\
6.5\end{array}$ & $\begin{array}{l}88 \\
83 \\
79\end{array}$ \\
\hline
\end{tabular}

301754096380801 SOMERVILLE LAKE SITE FC

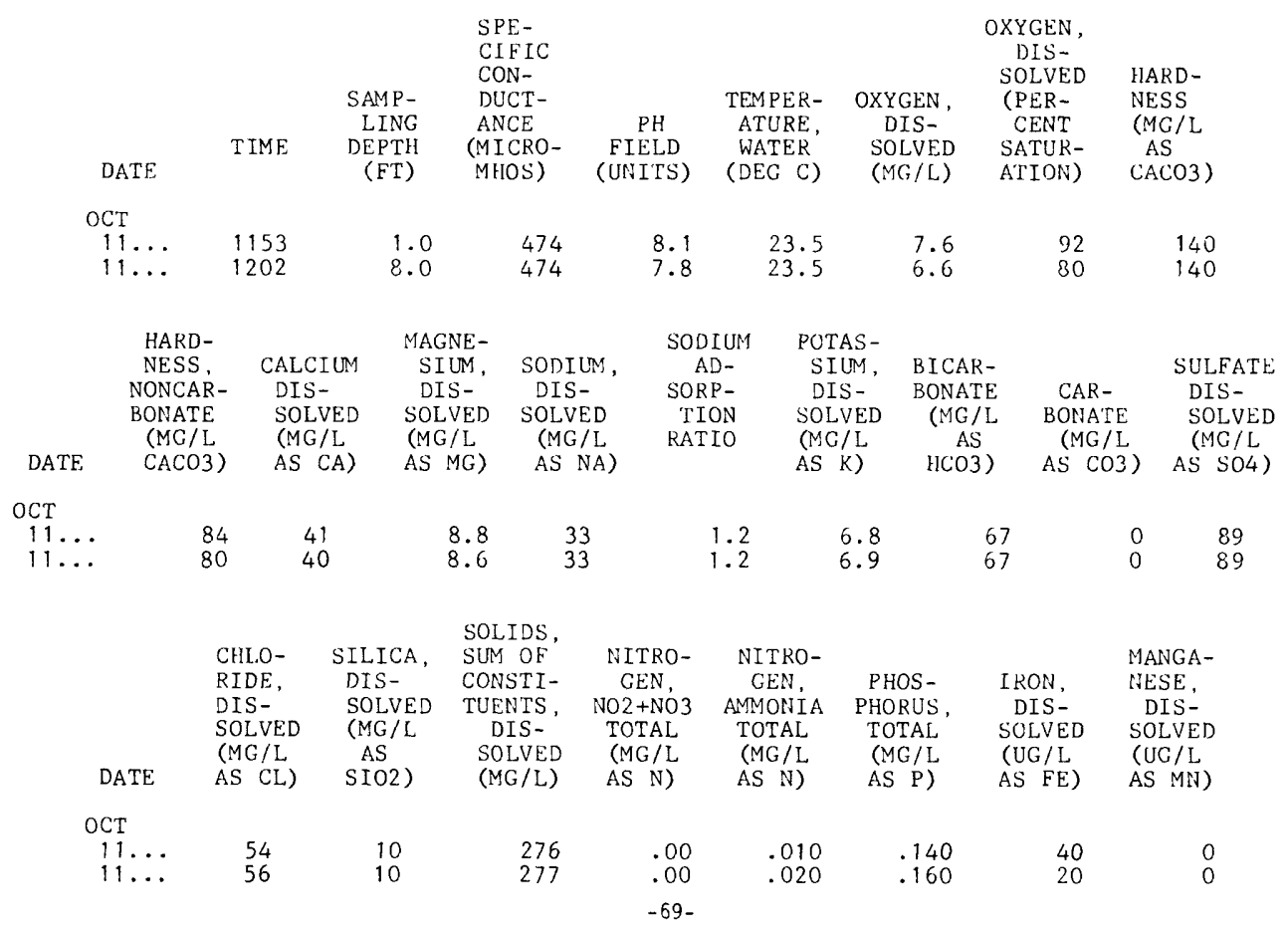


$\mathrm{FT}=$ feet; MICROMHOS = micromhos per centimeter at $25^{\circ}$ Celsius; ${ }^{\circ} \mathrm{C}-$ degrees Celsius; $\mathrm{MG} / \mathrm{L}=$ milligrams per liter; UG/L = micrograms per liter

301908096313101 SOMERVILLE LAKE SITE AC

\begin{tabular}{|c|c|c|c|c|c|c|c|c|c|}
\hline DATE & TIME & $\begin{array}{l}\text { SAMP- } \\
\text { LING } \\
\text { DEPTH } \\
(F \Gamma)\end{array}$ & $\begin{array}{l}\text { SPE- } \\
\text { CIFIC } \\
\text { CON- } \\
\text { DUCT- } \\
\text { ANCE } \\
\text { (MICRO- } \\
\text { MHOS) }\end{array}$ & $\begin{array}{c}\text { PH } \\
\text { FIELD } \\
\text { (UNITS) }\end{array}$ & $\begin{array}{c}\text { TEMPER- } \\
\text { ATURE, } \\
\text { WATER } \\
\text { (DEG C) }\end{array}$ & $\begin{array}{c}\text { TRANS- } \\
\text { PAR- } \\
\text { ENCY } \\
\text { (SECCHI } \\
\text { DISK) } \\
\text { (M) }\end{array}$ & $\begin{array}{c}\text { OXYGEN, } \\
\text { DIS- } \\
\text { SOLVED } \\
\text { (MG/L) }\end{array}$ & $\begin{array}{c}\text { OXYGEN, } \\
\text { DIS- } \\
\text { SOLVED } \\
\text { (PER- } \\
\text { CENT } \\
\text { SATUR- } \\
\text { ATION) }\end{array}$ & $\begin{array}{l}\text { HARD- } \\
\text { NESS } \\
(M G / L \\
\text { AS } \\
\text { CACO3) }\end{array}$ \\
\hline $\begin{array}{l}\text { ANI } \\
09 \ldots \\
09 \ldots \\
09 \ldots \\
09 \ldots\end{array}$ & $\begin{array}{l}1205 \\
1210 \\
1215 \\
1225\end{array}$ & $\begin{array}{r}1.0 \\
10.0 \\
20.0 \\
28.0\end{array}$ & $\begin{array}{l}445 \\
445 \\
445 \\
445\end{array}$ & $\begin{array}{l}7.6 \\
7.6 \\
7.6 \\
7.6\end{array}$ & $\begin{array}{l}5.5 \\
5.5 \\
5.5 \\
5.5\end{array}$ & $\begin{array}{l}.70 \\
-- \\
-- \\
--\end{array}$ & $\begin{array}{l}12.2 \\
12.2 \\
12.2 \\
12.1\end{array}$ & $\begin{array}{l}99 \\
99 \\
99 \\
98\end{array}$ & $\begin{array}{r}130 \\
-- \\
120\end{array}$ \\
\hline DATE & $\begin{array}{l}\text { HARD- } \\
\text { NESS } \\
\text { NONCAR- } \\
\text { BONATE } \\
\text { (MG/I, } \\
\text { CACO3) }\end{array}$ & $\begin{array}{l}\text { CALCIUM } \\
\text { DIS- } \\
\text { SOLVED } \\
\text { (MG/L } \\
\text { AS CA) }\end{array}$ & $\begin{array}{l}\text { MAGNE- } \\
\text { SIUM, } \\
\text { DIS- } \\
\text { SOLVED } \\
\text { (MC/L } \\
\text { AS MG) }\end{array}$ & $\begin{array}{l}\text { SODIUM, } \\
\text { DIS- } \\
\text { SOLVED } \\
\text { (MG/L } \\
\text { AS NA) }\end{array}$ & $\begin{array}{c}\text { SODIUM } \\
\text { AD- } \\
\text { SORP- } \\
\text { TION } \\
\text { RATIO }\end{array}$ & $\begin{array}{l}\text { POTAS- } \\
\text { SIUM, } \\
\text { DIS- } \\
\text { SOLVED } \\
\text { (MG/L } \\
\text { AS K) }\end{array}$ & $\begin{array}{c}\text { BICAR- } \\
\text { BONATE } \\
\text { (MG/L } \\
\text { AS } \\
\text { HCO3) }\end{array}$ & $\begin{array}{c}\text { CAR- } \\
\text { BONATE } \\
\text { (MG/L } \\
\text { AS CO3) }\end{array}$ & $\begin{array}{l}\text { SULFATE } \\
\text { DIS- } \\
\text { SOLVED } \\
\text { (MG/L } \\
\text { AS SO4) }\end{array}$ \\
\hline $\begin{array}{l}\text { JAN } \\
09 \ldots \\
09 \ldots \\
09 \ldots \\
09 \ldots\end{array}$ & $\begin{array}{l}77 \\
-- \\
-- \\
74\end{array}$ & $37^{--}$ & $\begin{array}{r}8.5 \\
-- \\
-- \\
8.2\end{array}$ & $31^{--}$ & $\begin{array}{r}1.2 \\
-- \\
-\overline{2} \\
1.2\end{array}$ & $\begin{array}{r}6.3 \\
-- \\
- \\
6.2\end{array}$ & $\begin{array}{l}61 \\
-- \\
\overline{61}\end{array}$ & $\begin{array}{r}0 \\
-- \\
-- \\
0\end{array}$ & 78 \\
\hline DATE. & $\begin{array}{l}\text { CHLO- } \\
\text { RIDE, } \\
\text { DIS- } \\
\text { SOLVED } \\
\text { (MC/L } \\
\text { AS CL) }\end{array}$ & $\begin{array}{l}\text { FLUO- } \\
\text { RIDE, } \\
\text { DIS- } \\
\text { SOLVED } \\
\text { (MG/L } \\
\text { AS F) }\end{array}$ & $\begin{array}{l}\text { SILICA, } \\
\text { DIS- } \\
\text { SOLVED } \\
\text { (MG/L } \\
\text { AS } \\
\text { SIO2) }\end{array}$ & $\begin{array}{l}\text { SOLIDS, } \\
\text { SUM OF } \\
\text { CONSTI- } \\
\text { TUENTS, } \\
\text { DIS- } \\
\text { SOLVED } \\
(\mathrm{MG} / \mathrm{L})\end{array}$ & $\begin{array}{c}\text { NITRO- } \\
\text { GEN, } \\
\text { NO2+NO3 } \\
\text { TOTAL } \\
\text { (MG/L } \\
\text { AS N) }\end{array}$ & $\begin{array}{l}\text { NITRO- } \\
\text { GEN, } \\
\text { AMMONIA } \\
\text { TOTAL } \\
\text { (MG/L } \\
\text { AS N) }\end{array}$ & $\begin{array}{c}\text { PHOS- } \\
\text { PHORUS, } \\
\text { TOTAL } \\
(M G / L \\
\text { AS P) }\end{array}$ & $\begin{array}{l}\text { IRON, } \\
\text { DIS- } \\
\text { SOLVED } \\
\text { (UG/L } \\
\text { AS FE) }\end{array}$ & $\begin{array}{l}\text { MANGA- } \\
\text { NESE, } \\
\text { DIS- } \\
\text { SOLVED } \\
\text { (UG/L } \\
\text { AS MN) }\end{array}$ \\
\hline JAN & & & & & & & & & \\
\hline $\begin{array}{l}09 \ldots \\
09 \ldots \\
09 \ldots\end{array}$ & $51^{--}$ & $\begin{array}{l}-- \\
--\end{array}$ & $\begin{array}{r}0.0 \\
-- \\
-- \\
8.6\end{array}$ & $\begin{array}{r}203 \\
-- \\
242\end{array}$ & .0 & $\begin{array}{r}.000 \\
.060\end{array}$ & $\begin{array}{r}.040 \\
.040\end{array}$ & 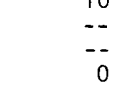 & -- \\
\hline
\end{tabular}

301940096315801 SOMERVILLE LAKE SITE AL

\begin{tabular}{|c|c|c|c|c|c|c|c|}
\hline & & & $\begin{array}{l}\text { SPE- } \\
\text { CIFIC } \\
\text { CON- }\end{array}$ & & & & $\begin{array}{c}\text { OXYGEN, } \\
\text { DIS- } \\
\text { SOLVED }\end{array}$ \\
\hline & & $\begin{array}{l}\text { SAMP- } \\
\text { LING }\end{array}$ & $\begin{array}{l}\text { DUCT- } \\
\text { ANCE }\end{array}$ & $\mathrm{PH}$ & $\begin{array}{l}\text { TEMPER- } \\
\text { ATURE, }\end{array}$ & $\begin{array}{l}\text { OXYGEN, } \\
\text { DIS- }\end{array}$ & $\begin{array}{l}\text { (PER- } \\
\text { CENT }\end{array}$ \\
\hline ATE & TIME & $\begin{array}{c}\text { DEPTH } \\
(\mathrm{FT})\end{array}$ & $\begin{array}{l}\text { (MICRO- } \\
\text { MHOS) }\end{array}$ & $\begin{array}{l}\text { FIEILD } \\
\text { (UNITS) }\end{array}$ & $\begin{array}{l}\text { WATER } \\
\text { (DEG C) }\end{array}$ & $\begin{array}{l}\text { SOLVED } \\
(\mathrm{MG} / \mathrm{L})\end{array}$ & $\begin{array}{l}\text { SATUR- } \\
\text { ATION) }\end{array}$ \\
\hline
\end{tabular}

\begin{tabular}{|c|c|c|c|c|c|c|c|}
\hline $\begin{array}{l}09 \ldots \\
09 \ldots \\
09 \ldots \\
09 \ldots\end{array}$ & $\begin{array}{l}1227 \\
1229 \\
1231 \\
1233\end{array}$ & $\begin{array}{r}1.0 \\
10.0 \\
20.0 \\
29.0\end{array}$ & $\begin{array}{l}445 \\
445 \\
445 \\
445\end{array}$ & $\begin{array}{l}7.6 \\
7.6 \\
7.6\end{array}$ & $\begin{array}{l}5.5 \\
5.5 \\
5.5 \\
5.5\end{array}$ & $\begin{array}{l}12.0 \\
11.9 \\
11.8\end{array}$ & $\begin{array}{l}98 \\
97 \\
96 \\
96\end{array}$ \\
\hline
\end{tabular}

302026096341501 SOMERVILLE LAKE SITE BC

\begin{tabular}{|c|c|c|c|c|c|c|c|}
\hline DATE & TIME & $\begin{array}{l}\text { SAMP- } \\
\text { LING } \\
\text { DEPTH } \\
\text { (FT) }\end{array}$ & $\begin{array}{l}\text { SPE- } \\
\text { CIFIC } \\
\text { CON- } \\
\text { DUCT- } \\
\text { ANCE } \\
\text { (MICRO- } \\
\text { MHOS) }\end{array}$ & $\begin{array}{c}\text { PH } \\
\text { FIELD } \\
\text { (UNITS) }\end{array}$ & $\begin{array}{c}\text { TEMPER- } \\
\text { ATURE, } \\
\text { WATER } \\
\text { (DEG C) }\end{array}$ & $\begin{array}{c}\text { TRANS- } \\
\text { PAR- } \\
\text { ENCY } \\
\text { (SECCHI } \\
\text { DISK) } \\
(M)\end{array}$ & $\begin{array}{c}\text { OXYGEN, } \\
\text { DIS- } \\
\text { SOLVED } \\
\text { (NG/L) }\end{array}$ \\
\hline \multicolumn{8}{|l|}{ JAN } \\
\hline $\begin{array}{l}09 \ldots \\
09 \ldots\end{array}$ & $\begin{array}{l}1246 \\
1255\end{array}$ & $\begin{array}{r}1.0 \\
13.0\end{array}$ & $\begin{array}{l}424 \\
424\end{array}$ & $\begin{array}{l}7.6 \\
7.6\end{array}$ & $\begin{array}{l}5.0 \\
5.0\end{array}$ & .60 & $\begin{array}{l}12.4 \\
12.6\end{array}$ \\
\hline
\end{tabular}


Table 12--CHEMICAL-QUALITY SURVEYS OF SOMERVILLE LAKE JANUARY 9, 1979--Continued

302026096341501 SOMERVILLE LAKE SITE BC--Continued

\begin{tabular}{ccccccc}
\multicolumn{1}{c}{ OXYGEN, } & \multicolumn{1}{c}{} & & \\
DIS- & NITRO- & NITRO- & & & MANGA- \\
SOLVED & GEN, & GEN, & PHOS- & IRON, & NESE, \\
& (PER- & NO2+NO3 & AMMONIA & PHORUS, & DIS- & DIS- \\
& CENT & TOTAL & TOTAL & TOTAL & SOLVED & SOLVED \\
& SATUR- & (MG/L & (MG/L & (MG/L & (UG/L & (UG/L \\
DATE & ATION) & AS N) & AS N) & AS P) & AS FE) & AS MN)
\end{tabular}

\begin{tabular}{|c|c|c|c|c|c|}
\hline $\begin{array}{l}09 \ldots \\
09 \ldots\end{array}$ & $\begin{array}{r}99 \\
101\end{array}$ & $\begin{array}{l}.05 \\
.05\end{array}$ & $\begin{array}{l}.080 \\
.100\end{array}$ & $\begin{array}{l}.050 \\
.060\end{array}$ & $\begin{array}{l}10 \\
10\end{array}$ \\
\hline
\end{tabular}

301805096332501 SOMERVILLE LAKE SITE CC

\begin{tabular}{|c|c|c|c|c|c|c|c|}
\hline DATE & TIME & $\begin{array}{l}\text { SAMP- } \\
\text { LING } \\
\text { DEPTH } \\
\text { (FT) }\end{array}$ & $\begin{array}{l}\text { SPE- } \\
\text { CIFIC } \\
\text { CON- } \\
\text { DUCT- } \\
\text { ANCE } \\
\text { (MICRO- } \\
\text { MHOS) }\end{array}$ & $\begin{array}{c}\text { PH } \\
\text { FIELD } \\
\text { (UNI'TS) }\end{array}$ & $\begin{array}{c}\text { TEMPER- } \\
\text { ATURE, } \\
\text { WATER } \\
\left(\begin{array}{ll}\text { DEG } & \text { C) }\end{array}\right.\end{array}$ & $\begin{array}{c}\text { TRANS- } \\
\text { PAR- } \\
\text { ENCY } \\
\text { (SECCHI } \\
\text { DISK) } \\
\text { (M) }\end{array}$ & $\begin{array}{c}\text { OXYGEN, } \\
\text { DIS- } \\
\text { SOLVED } \\
(\mathrm{MG} / \mathrm{L})\end{array}$ \\
\hline \multicolumn{8}{|c|}{ JAN } \\
\hline $\begin{array}{l}09 \ldots \\
09 . . .\end{array}$ & $\begin{array}{l}1342 \\
1350\end{array}$ & $\begin{array}{r}1.0 \\
10.0\end{array}$ & $\begin{array}{l}445 \\
445\end{array}$ & $\begin{array}{l}7.6 \\
7.6\end{array}$ & $\begin{array}{l}5.5 \\
5.0\end{array}$ & $\begin{array}{r}.70 \\
--\end{array}$ & $\begin{array}{l}12.2 \\
12.0\end{array}$ \\
\hline
\end{tabular}

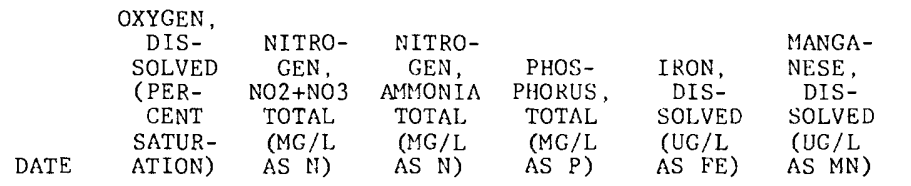

\begin{tabular}{|c|c|c|c|c|c|c|}
\hline $\begin{array}{l}09 \ldots \\
09 \ldots\end{array}$ & $\begin{array}{l}99 \\
96\end{array}$ & $\begin{array}{l}.05 \\
.04\end{array}$ & $\begin{array}{l}.100 \\
.040\end{array}$ & $\begin{array}{l}.040 \\
.050\end{array}$ & $\begin{array}{l}0 \\
0\end{array}$ & 20 \\
\hline
\end{tabular}

301847096334601 SOMERVILLE LAKE SITE DR

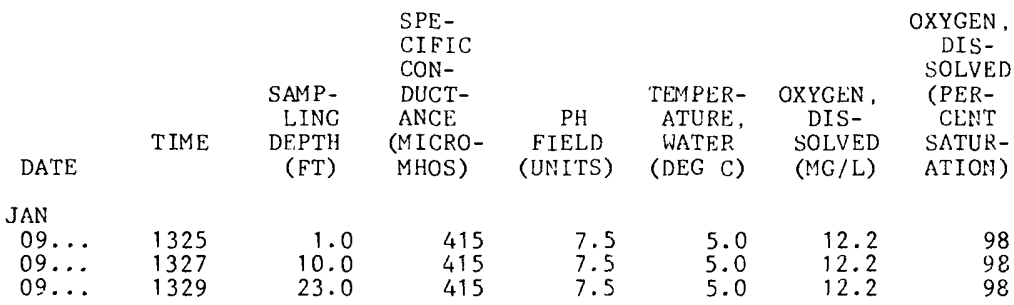

301904096335601 SOMERVILLE LAKE SITE DC

\begin{tabular}{|c|c|c|c|c|c|c|c|c|c|}
\hline DATE & TIME & $\begin{array}{l}\text { SAMP- } \\
\text { LING } \\
\text { DEPTH } \\
\text { (FT) }\end{array}$ & $\begin{array}{l}\text { SPE- } \\
\text { CIFIC } \\
\text { CON- } \\
\text { DUGT- } \\
\text { ANCE } \\
\text { (MICRO- } \\
\text { MHOS) }\end{array}$ & $\begin{array}{c}\text { PH } \\
\text { FIELD } \\
\text { (UNITS) }\end{array}$ & $\begin{array}{l}\text { TEMPER- } \\
\text { ATURE, } \\
\text { WATFR } \\
\text { (DEG C) }\end{array}$ & $\begin{array}{c}\text { TRANS- } \\
\text { PAR- } \\
\text { ENCY } \\
\text { (SECCHI } \\
\text { DISK) } \\
\text { (M) }\end{array}$ & $\begin{array}{c}\text { OXYGEN, } \\
\text { DIS- } \\
\text { SOLVED } \\
(M G / L)\end{array}$ & $\begin{array}{l}\text { OXYGEN, } \\
\text { DIS- } \\
\text { SOLVED } \\
\text { (PER- } \\
\text { CENT } \\
\text { SATUR- } \\
\text { ATION) }\end{array}$ & $\begin{array}{l}\text { HARD- } \\
\text { NESS } \\
\text { (MG/L } \\
\text { AS } \\
\text { CACO3) }\end{array}$ \\
\hline \multicolumn{10}{|l|}{ JAN } \\
\hline $\begin{array}{l}09 \ldots \\
09 \ldots \\
09 \ldots \\
09 \ldots\end{array}$ & $\begin{array}{l}1303 \\
1308 \\
1311 \\
1317\end{array}$ & $\begin{array}{r}1.0 \\
10.0 \\
20.0 \\
28.0\end{array}$ & $\begin{array}{l}415 \\
415 \\
415 \\
415\end{array}$ & $\begin{array}{l}7.5 \\
7.5 \\
7.5 \\
7.5\end{array}$ & $\begin{array}{l}5.0 \\
5.0 \\
5.0 \\
5.0\end{array}$ & $\begin{array}{c}.50 \\
-- \\
-- \\
--\end{array}$ & $\begin{array}{l}12.2 \\
12.1 \\
12.1 \\
11.9\end{array}$ & $\begin{array}{l}98 \\
97 \\
97 \\
95\end{array}$ & $\begin{array}{c}110 \\
-- \\
110\end{array}$ \\
\hline
\end{tabular}


Table 12--CHEMICAL-QUALITY SURVEYS OF SOMERVILLE LAKE JANUARY 9, 1979--Continued

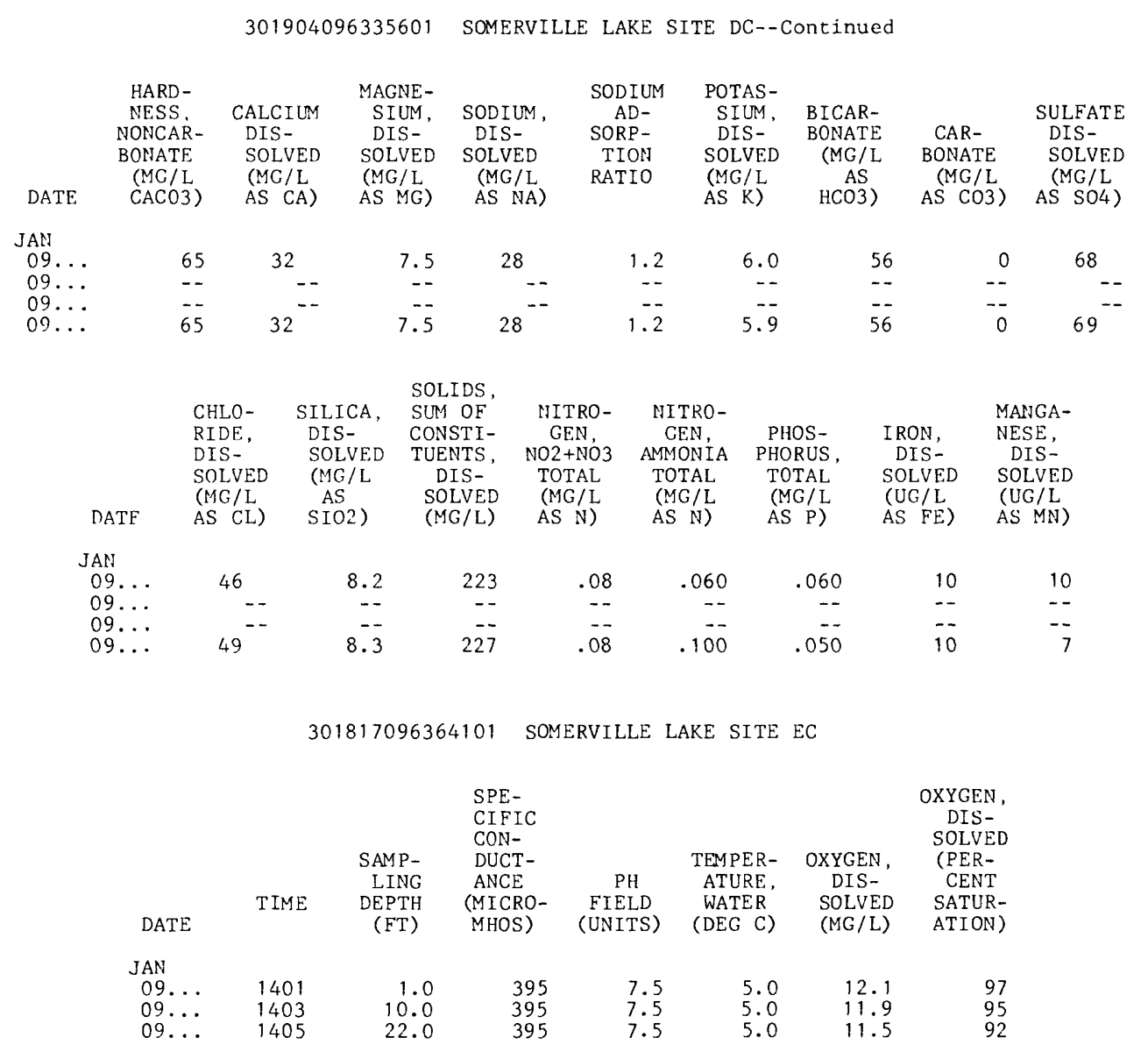




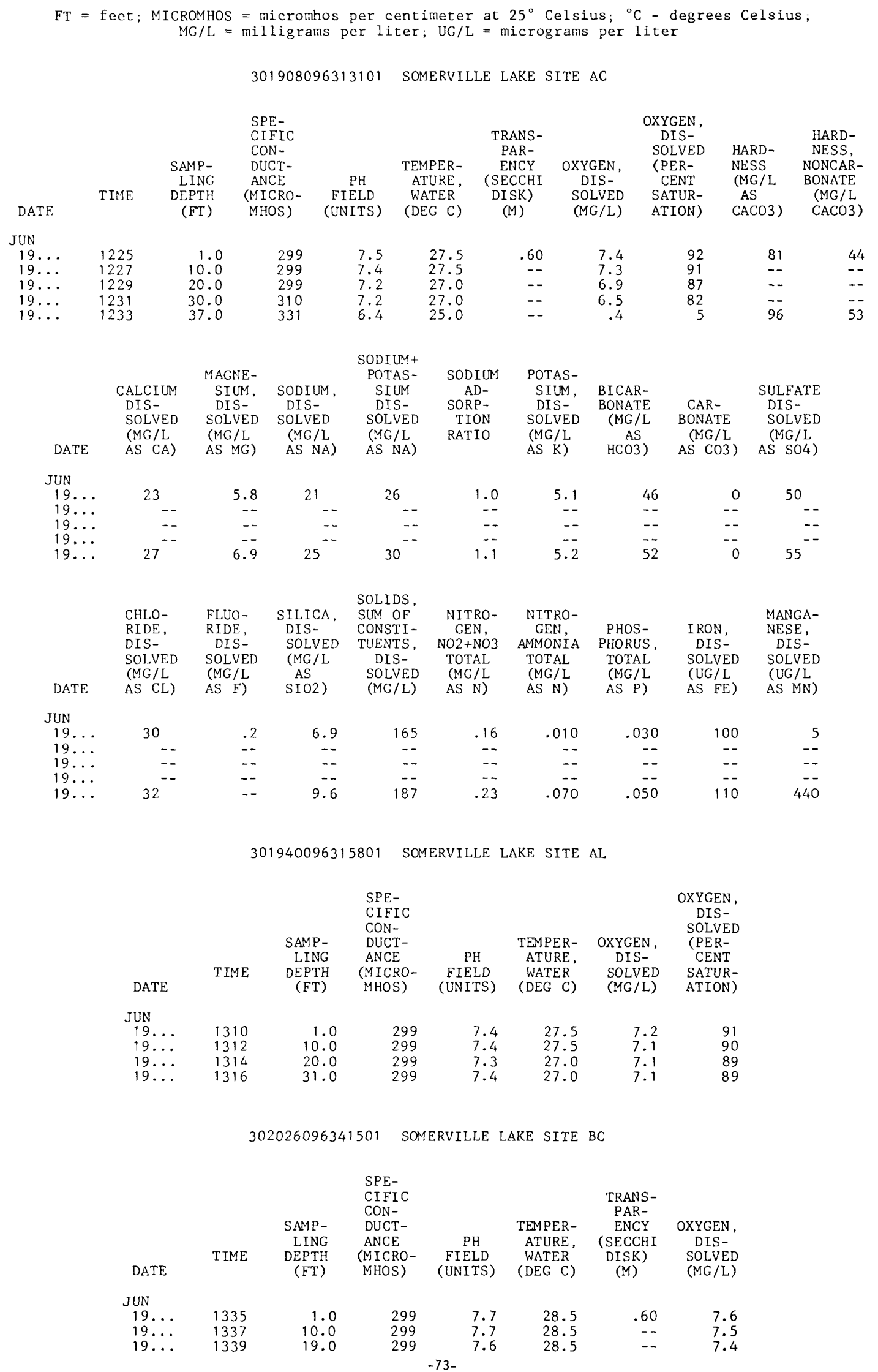


Table 13--CHEMICAL-QUALITY SURVEYS OF SOMERVILLE LAKE JUNE 19, 1979--Continued

302026096341501 SOMERVILLE LAKE SITE BC--Continued

\begin{tabular}{ccccccc}
\multicolumn{1}{c}{ OXYGEN, } & & & & \\
DIS- & NITRO- & NITRO- & & MANGA- \\
SOLVED & GEN, & GEN, & PHOS- & IRON, & NESE, \\
& (PER- & NO2+NO3 & AMMONIA & PHORUS, & DIS- & DIS- \\
CENT & TOTAL & TOTAL & TOTAL & SOLVED & SOLVED \\
& SATUR- & (MG/L & (MG/L & (MG/L & (UG/L & (UG/L \\
DATE & ATION) & AS N) & AS N) & AS P) & AS FE) & AS MN)
\end{tabular}

$\begin{array}{rrrrrrr}\text { JUN } & & & & & & \\ 19 \ldots & 98 & .06 & .030 & .030 & 60 & 10 \\ 19 \ldots & 97 & -\overline{-} & .040 & .030 & 70 & -- \\ 19 \ldots & 95 & .08 & .040\end{array}$

301805096332501 SOMERVILLE LAKE SITE CC

\begin{tabular}{|c|c|c|c|c|c|c|c|}
\hline DATE & TIME & $\begin{array}{c}\text { SAMP- } \\
\text { LING } \\
\text { DEPTH } \\
\text { (FT) }\end{array}$ & $\begin{array}{l}\text { SPE- } \\
\text { CIFIC } \\
\text { CON- } \\
\text { DUCT- } \\
\text { ANCE } \\
\text { (MICRO- } \\
\text { MHOS) }\end{array}$ & $\begin{array}{c}\text { PH } \\
\text { FIE.LD } \\
\text { (UNITS) }\end{array}$ & 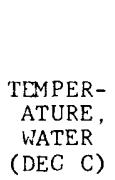 & $\begin{array}{l}\text { TRANS- } \\
\text { PAR- } \\
\text { ENCY } \\
\text { (SECCHI } \\
\text { DISK) } \\
(M)\end{array}$ & $\begin{array}{c}\text { OXYGEN, } \\
\text { DIS- } \\
\text { SOLVED } \\
\text { (MG/L) }\end{array}$ \\
\hline \multicolumn{8}{|l|}{ JUN } \\
\hline $\begin{array}{l}19 \ldots \\
19 \ldots \\
19 \ldots\end{array}$ & $\begin{array}{l}1540 \\
1542 \\
1544\end{array}$ & $\begin{array}{r}1.0 \\
10.0 \\
20.0\end{array}$ & $\begin{array}{l}308 \\
308 \\
308\end{array}$ & $\begin{array}{l}7.1 \\
7.1 \\
6.8\end{array}$ & $\begin{array}{l}27.0 \\
27.0 \\
26.5\end{array}$ & $\begin{array}{l}.60 \\
-- \\
--\end{array}$ & $\begin{array}{l}6.6 \\
6.5 \\
4.4\end{array}$ \\
\hline
\end{tabular}

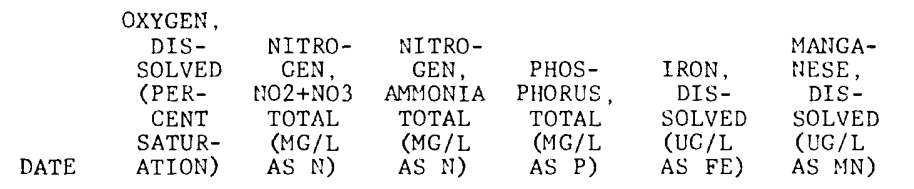

\begin{tabular}{|c|c|c|c|c|c|c|}
\hline JUN & & & & & & \\
\hline $19 \ldots$ & 84 & .21 & .020 & .020 & 80 & 20 \\
\hline $19 \ldots$ & 82 & - & -- & -- & $-\overline{0}$ & 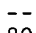 \\
\hline $19 \ldots$ & 55 & .21 & .040 & .030 & 150 & 8 \\
\hline
\end{tabular}

301847096334601 SOMERVILLE LAKE SITE DR

\begin{tabular}{|c|c|c|c|c|c|c|c|}
\hline DATE & TIME & $\begin{array}{c}\text { SAMP- } \\
\text { LING } \\
\text { DEPTH } \\
\text { (FT) }\end{array}$ & $\begin{array}{l}\text { SPE- } \\
\text { CIFIC } \\
\text { CON- } \\
\text { DUCT- } \\
\text { ANCE } \\
\text { (MICRO- } \\
\text { MHOS) }\end{array}$ & $\begin{array}{c}\text { PH } \\
\text { FIELD } \\
\text { (UNITS) }\end{array}$ & $\begin{array}{c}\text { TEMPER- } \\
\text { ATURE, } \\
\text { WATER } \\
\text { (DEG C) }\end{array}$ & $\begin{array}{c}\text { OXYGEN, } \\
\text { DIS- } \\
\text { SOLVED } \\
\text { (MG } / L)\end{array}$ & $\begin{array}{l}\text { OXYGEN, } \\
\text { DIS- } \\
\text { SOLVED } \\
\text { (PER- } \\
\text { CENT } \\
\text { SATUR- } \\
\text { ATION) }\end{array}$ \\
\hline \multicolumn{8}{|l|}{ JUN } \\
\hline $\begin{array}{l}19 \ldots \\
19 \ldots \\
19 \ldots \\
19 \ldots\end{array}$ & $\begin{array}{l}1415 \\
1417 \\
1419 \\
1421\end{array}$ & $\begin{array}{r}1.0 \\
10.0 \\
20.0 \\
32.0\end{array}$ & $\begin{array}{l}258 \\
268 \\
268 \\
308\end{array}$ & $\begin{array}{l}7.3 \\
7.2 \\
7.1 \\
6.6\end{array}$ & $\begin{array}{l}27.5 \\
27.5 \\
27.0 \\
26.0\end{array}$ & $\begin{array}{l}7.0 \\
6.9 \\
6.3 \\
2.1\end{array}$ & $\begin{array}{l}89 \\
87 \\
79 \\
26\end{array}$ \\
\hline
\end{tabular}

301904096335601 SOMERVILLE IAAKE SITE DC

\begin{tabular}{|c|c|c|c|c|c|c|c|c|c|}
\hline DATE & TIME & $\begin{array}{l}\text { SAMP- } \\
\text { LING } \\
\text { DEPTH } \\
\text { (FT) }\end{array}$ & $\begin{array}{l}\text { SPE- } \\
\text { CIFIC } \\
\text { CON- } \\
\text { DUCT- } \\
\text { ANCE } \\
\text { (MICRO- } \\
\text { MHOS) }\end{array}$ & $\begin{array}{c}\text { PH } \\
\text { FIELD } \\
\text { (UNITS) }\end{array}$ & $\begin{array}{c}\text { TEMPER- } \\
\text { ATURE, } \\
\text { WATER } \\
\text { (DEG C) }\end{array}$ & $\begin{array}{l}\text { TRANS- } \\
\text { PAR- } \\
\text { ENCY } \\
\text { (SECCHI } \\
\text { DISK) } \\
(M)\end{array}$ & $\begin{array}{c}\text { OXYGEN } \\
\text { DIS- } \\
\text { SOLVED } \\
(M G / L)\end{array}$ & $\begin{array}{c}\text { OXYGEN, } \\
\text { DIS- } \\
\text { SOLVED } \\
\text { (PER- } \\
\text { CENT } \\
\text { SATUR- } \\
\text { ATION) }\end{array}$ & $\begin{array}{l}\text { HARD- } \\
\text { NESS } \\
(\mathrm{MG} / \mathrm{L} \\
\mathrm{AS} \\
\mathrm{CACO} 3)\end{array}$ \\
\hline \multicolumn{10}{|l|}{ JUN } \\
\hline $\begin{array}{l}19 \ldots \\
19 \ldots \\
19 \ldots \\
19 \ldots\end{array}$ & $\begin{array}{l}1350 \\
1352 \\
1354 \\
1356\end{array}$ & $\begin{array}{r}1.0 \\
10.0 \\
20.0 \\
34.0\end{array}$ & $\begin{array}{l}258 \\
268 \\
268 \\
314\end{array}$ & $\begin{array}{l}7.5 \\
7.3 \\
7.3 \\
6.6\end{array}$ & $\begin{array}{l}28.0 \\
27.5 \\
27.5 \\
26.0\end{array}$ & $\begin{array}{c}.50 \\
-- \\
-- \\
--\end{array}$ & $\begin{array}{l}7.5 \\
6.9 \\
6.9 \\
1.4\end{array}$ & $\begin{array}{l}96 \\
87 \\
87 \\
17\end{array}$ & $\begin{array}{l}74 \\
-- \\
-\overline{88}\end{array}$ \\
\hline
\end{tabular}


Table 13--CHEMICAL-QUALITY SURVEYS OF SOMERVILLE LAKE JUNE 19, 1979--Continued

301904096335601 SOMERVILLE LAKE SITE DC--Continued

\begin{tabular}{|c|c|c|c|c|c|c|c|c|c|}
\hline & $\begin{array}{c}\text { HARD- } \\
\text { NESS, } \\
\text { NONCAR- } \\
\text { BONATE } \\
\text { (MG/L }\end{array}$ & $\begin{array}{l}\text { CALCIUM } \\
\text { DIS- } \\
\text { SOLVED } \\
\text { (MG/L }\end{array}$ & $\begin{array}{l}\text { MAGNE- } \\
\text { SIUM, } \\
\text { DIS- } \\
\text { SOLVED } \\
\text { (MG/L }\end{array}$ & $\begin{array}{l}\text { SODIUM, } \\
\text { DIS- } \\
\text { SOLVED } \\
\text { (MG/L }\end{array}$ & $\begin{array}{c}\text { SODIUM+ } \\
\text { POTAS- } \\
\text { SIUM } \\
\text { DIS- } \\
\text { SOLVED } \\
\text { (MG/L }\end{array}$ & $\begin{array}{c}\text { SODIUM } \\
\text { AD- } \\
\text { SORP- } \\
\text { TION } \\
\text { RATIO }\end{array}$ & $\begin{array}{l}\text { POTAS- } \\
\text { SIUM, } \\
\text { DIS- } \\
\text { SOLVED } \\
\text { (MG/L }\end{array}$ & $\begin{array}{c}\text { BICAR- } \\
\text { BONATE } \\
\text { (MG/L } \\
\text { AS }\end{array}$ & $\begin{array}{l}\text { CAR- } \\
\text { BONATE } \\
\text { (MG / L }\end{array}$ \\
\hline $\mathrm{ATE}$ & (ACO3) & AS (A) & AS MG) & AS NA) & AS NA) & & AS $\mathrm{K}$ & $\mathrm{HCO} 3)$ & $\mathrm{AS}(\mathrm{CO})$ \\
\hline
\end{tabular}

JUN

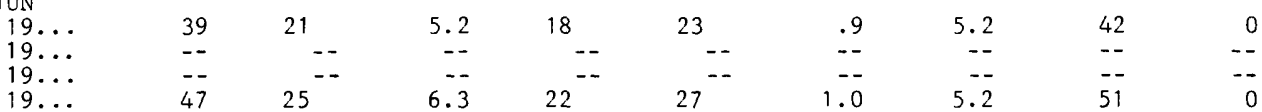

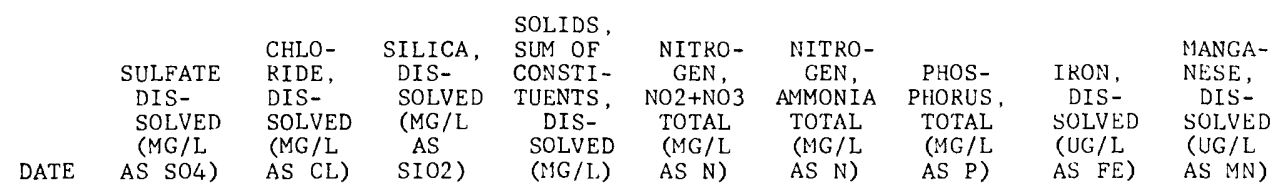

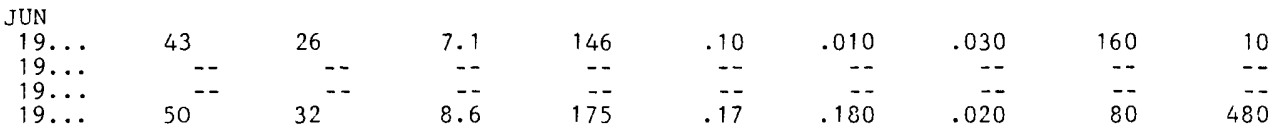

301817096364101 SOMERVILLE LAKE SITE EC

\begin{tabular}{|c|c|c|c|c|c|c|c|}
\hline DATE & TIME & $\begin{array}{l}\text { SAMP- } \\
\text { LING } \\
\text { DEPTH } \\
\text { (FT) }\end{array}$ & $\begin{array}{l}\text { SPE- } \\
\text { CIFIC } \\
\text { CON- } \\
\text { DUCT- } \\
\text { ANCE } \\
\text { (MICRO- } \\
\text { MHOS) }\end{array}$ & $\begin{array}{c}\text { PH } \\
\text { FIELD } \\
\text { (UNI'TS) }\end{array}$ & $\begin{array}{c}\text { TEMPER- } \\
\text { ATURE, } \\
\text { WATER } \\
\text { (DEG } \quad \text { C) }\end{array}$ & $\begin{array}{c}\text { OXYGEN, } \\
\text { DIS- } \\
\text { SOLVED } \\
\text { (MG/L) }\end{array}$ & $\begin{array}{c}\text { OXYGEN, } \\
\text { DIS- } \\
\text { SOLVED } \\
\text { (PER- } \\
\text { CENT } \\
\text { SATUR- } \\
\text { ATION) }\end{array}$ \\
\hline \multicolumn{8}{|l|}{ JUN } \\
\hline $\begin{array}{l}19 \ldots \\
19 \ldots \\
19 \ldots \\
19 \ldots\end{array}$ & $\begin{array}{l}1435 \\
1437 \\
1439 \\
1441\end{array}$ & $\begin{array}{r}1.0 \\
10.0 \\
20.0 \\
30.0\end{array}$ & $\begin{array}{l}266 \\
266 \\
278 \\
290\end{array}$ & $\begin{array}{l}7.0 \\
7.0 \\
6.9 \\
6.8\end{array}$ & $\begin{array}{l}27.0 \\
27.0 \\
27.0 \\
26.5\end{array}$ & $\begin{array}{l}6.3 \\
6.2 \\
5.9 \\
5.2\end{array}$ & $\begin{array}{l}79 \\
78 \\
74 \\
65\end{array}$ \\
\hline
\end{tabular}

301754096380801 SOMERVILLE LAKE SITE FC

\begin{tabular}{|c|c|c|c|c|c|c|c|c|c|}
\hline DATE & T IME & $\begin{array}{l}\text { SAMP- } \\
\text { LING } \\
\text { DEPTH } \\
\text { (FT) }\end{array}$ & $\begin{array}{l}\text { SPE- } \\
\text { CIFIC } \\
\text { CON- } \\
\text { DUCT- } \\
\text { ANCE } \\
\text { (MICRO- } \\
\text { MHOS) }\end{array}$ & $\begin{array}{c} \\
\text { PH } \\
\text { FIELD } \\
\text { (UNI'TS) }\end{array}$ & $\begin{array}{l}\text { TEMPER- } \\
\text { ATURE, } \\
\text { WATER } \\
\text { (DEG C) }\end{array}$ & $\begin{array}{l}\text { TRANS- } \\
\text { PAR- } \\
\text { ENCY } \\
\text { (SECCHI } \\
\text { DISK) } \\
\text { (M) }\end{array}$ & $\begin{array}{c}\text { OXYGEN, } \\
\text { DIS- } \\
\text { SOLVED } \\
\text { (MG/L) }\end{array}$ & $\begin{array}{c}\text { OXYGEN, } \\
\text { DIS- } \\
\text { SOLVED } \\
\text { (PER- } \\
\text { CENT } \\
\text { SATUR- } \\
\text { ATION) }\end{array}$ & $\begin{array}{l}\text { HARD- } \\
\text { NESS } \\
\text { (MG/L } \\
\text { AS } \\
\text { CACO3) }\end{array}$ \\
\hline $\begin{array}{l}\text { JUN } \\
19 \ldots \\
19 \ldots \\
19 \ldots\end{array}$ & $\begin{array}{l}1505 \\
1507 \\
1509\end{array}$ & $\begin{array}{r}1.0 \\
10.0 \\
20.0\end{array}$ & $\begin{array}{l}205 \\
205 \\
238\end{array}$ & $\begin{array}{l}6.8 \\
6.8 \\
6.6\end{array}$ & $\begin{array}{l}28.0 \\
27.5 \\
26.5\end{array}$ & $\begin{array}{c}.30 \\
-- \\
--\end{array}$ & $\begin{array}{l}5.6 \\
5.3 \\
3.4\end{array}$ & $\begin{array}{l}71 \\
67 \\
42\end{array}$ & $\begin{array}{l}59 \\
-67\end{array}$ \\
\hline DATE & $\begin{array}{c}\text { HARD- } \\
\text { NESS, } \\
\text { NONCAR- } \\
\text { BONATE } \\
\text { (MG / L } \\
\text { CACO3) }\end{array}$ & $\begin{array}{l}\text { CALCIUM } \\
\text { DIS- } \\
\text { SOLVED } \\
\text { (MG/L } \\
\text { AS CA) }\end{array}$ & $\begin{array}{l}\text { MAGNE- } \\
\text { SIUM, } \\
\text { DIS- } \\
\text { SOLVED } \\
\text { (MG/L } \\
\text { AS MG) }\end{array}$ & $\begin{array}{l}\text { SODIUM, } \\
\text { DIS- } \\
\text { SOLVED } \\
\text { (MG } / L \\
\text { AS NA) }\end{array}$ & $\begin{array}{c}\text { SODIUM+ } \\
\text { POTAS- } \\
\text { SIUM } \\
\text { DIS- } \\
\text { SOLVED } \\
\text { (MG/L } \\
\text { AS NA) }\end{array}$ & $\begin{array}{c}\text { SODIUM } \\
\text { AD- } \\
\text { SORP- } \\
\text { TION } \\
\text { RATIO }\end{array}$ & $\begin{array}{l}\text { POTAS- } \\
\text { SIUM, } \\
\text { DIS- } \\
\text { SOLVED } \\
\text { (MG / L } \\
\text { AS K) }\end{array}$ & $\begin{array}{c}\text { BICAR } \\
\text { BONATE } \\
\text { (MG / L } \\
\text { AS } \\
\text { HCO3) }\end{array}$ & $\begin{array}{c}\text { CAR- } \\
\text { BONATE } \\
(\mathrm{MG} / \mathrm{L} \\
\text { AS CO3) }\end{array}$ \\
\hline
\end{tabular}

\begin{tabular}{|c|c|c|c|c|c|c|c|c|c|}
\hline JUN & & & & & & & & & \\
\hline $19 \ldots$ & 22 & 17 & 3.9 & 12 & 16 & .7 & 4.4 & 44 & 0 \\
\hline $19 \ldots$ & $\overline{31}$ & $0^{--}$ & $-\overline{8}$ & - & -- & -- & -- & - & -- \\
\hline $19 \ldots$ & 31 & 19 & 4.8 & 16 & 21 & .9 & 4.6 & 44 & 0 \\
\hline
\end{tabular}


Table 13--CHEMICAL-QUALITY SURVEYS OF SOMERVILLE LAKE JUNE 19, 1979--Continued

301754096380801 SOMERVILLE LAKE SITE FC--Continued

$\begin{array}{llll}\text { CHLO- } & \text { SOLIDS, } & & \\ \text { SLM OF } & \text { NITRO- NITRO- } & \text { MANGA- }\end{array}$

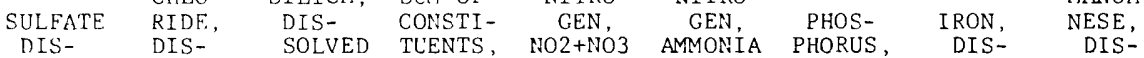

SOLVED SOLVED (MG/L DIS- TOTAL TOTAL TOTAL SOLVED SOLVED

(MG/L (MG/L AS SOLVED (MG/L (MG/L (MG/L (UG/L ) (UG/L)

DATE AS SO4) AS (L) SIO2) (MG/L) AS N) AS N) AS P) AS FE) AS MN)

\begin{tabular}{|c|c|c|c|c|c|c|c|c|c|}
\hline 19. & & & & & & & & & \\
\hline $10 \ldots$ & $-\cdots$ & -0 & 2.8 & 180 & - & 0 & _- & $0<0$ & 130 \\
\hline $19 \ldots$ & 37 & 23 & 8.6 & 135 & 23 & .070 & 050 & 170 & 22 \\
\hline
\end{tabular}


$\mathrm{FT}=$ feet $;$ MICROMHOS = inicromhos per centimeter at $25^{\circ}$ Celsius; ${ }^{\circ} \mathrm{C}$ - degrees Celsius; $M G / L=$ milligrams per liter; UG/L = micrograms per liter

301908096313101 SOMERVILLE LAKE SITE AC

\begin{tabular}{|c|c|c|c|c|c|c|c|c|c|c|}
\hline DATF & TIME & $\begin{array}{l}\text { SANP- } \\
\text { LING } \\
\text { DEPTH } \\
\text { (FT) }\end{array}$ & $\begin{array}{l}\text { SPE- } \\
\text { CIFIC } \\
\text { CON- } \\
\text { DUCT- } \\
\text { ANCE } \\
\text { (MICRO- } \\
\text { MHOS) }\end{array}$ & $\begin{array}{c}\text { PH } \\
\text { FIELD } \\
\text { (UNITS) }\end{array}$ & $\begin{array}{c}\text { TEMPER- } \\
\text { ATURE } \\
\text { WATER } \\
\text { (DEC C) }\end{array}$ & $\begin{array}{c}\text { TRANS- } \\
\text { PAR- } \\
\text { ENCY } \\
\text { (SECCHI } \\
\text { DISK) } \\
\text { (M) }\end{array}$ & $\begin{array}{c}\text { OXYGEN, } \\
\text { DIS- } \\
\text { SOLVED } \\
(\mathrm{MG} / \mathrm{L})\end{array}$ & $\begin{array}{c}\text { OXYGEN, } \\
\text { DIS- } \\
\text { SOLVED } \\
\text { (PER- } \\
\text { CENT } \\
\text { SATUR- } \\
\text { ATION) }\end{array}$ & $\begin{array}{l}\text { HARD- } \\
\text { NESS } \\
\text { (MG/L } \\
\text { AS } \\
\text { CACO } 3)\end{array}$ & $\begin{array}{c}\text { HARD- } \\
\text { NESS, } \\
\text { NONCAR- } \\
\text { BONATE } \\
\text { (MG/L } \\
\text { CACO3) }\end{array}$ \\
\hline \multicolumn{11}{|l|}{ AUG } \\
\hline $03 \ldots$ & 1310 & 1.0 & 279 & 8.4 & 31.0 & 1.10 & 8.7 & 116 & 85 & \\
\hline & 1314 & 10.0 & 279 & 8 & 31. & -. & & 115 & -- & \\
\hline & 1318 & 20.0 & 279 & 8.2 & 30 & -- & & 107 & -- & \\
\hline $03 \ldots$ & 1322 & 29.0 & 279 & 7.7 & 30.5 & - & 6.4 & 84 & 85 & \\
\hline
\end{tabular}

\begin{tabular}{|c|c|c|c|c|c|c|c|c|c|}
\hline & $\begin{array}{l}\text { CALCIUM } \\
\text { DIS- } \\
\text { SOLVED } \\
\text { (MG/L }\end{array}$ & $\begin{array}{l}\text { MAGNE- } \\
\text { SIUM, } \\
\text { DIS- } \\
\text { SOLVED } \\
\text { (MG/L }\end{array}$ & $\begin{array}{l}\text { SODIUM, } \\
\text { DIS- } \\
\text { SOLVED } \\
\text { (MG/L }\end{array}$ & $\begin{array}{l}\text { SODIUM+ } \\
\text { POTAS- } \\
\text { SIUM } \\
\text { DIS- } \\
\text { SOLVED } \\
\text { (MC/I. }\end{array}$ & $\begin{array}{l}\text { SODIUM } \\
\text { AD- } \\
\text { SORP- } \\
\text { TION } \\
\text { RATIO }\end{array}$ & $\begin{array}{l}\text { POTAS- } \\
\text { SIUM, } \\
\text { DIS- } \\
\text { SOLVED } \\
\text { (MG/L }\end{array}$ & $\begin{array}{c}\text { BICAR- } \\
\text { BONATE } \\
\text { (MG/L } \\
\text { AS }\end{array}$ & $\begin{array}{l}\text { CAR- } \\
\text { BONATE } \\
\text { (MG/L }\end{array}$ & $\begin{array}{l}\text { SULFATE } \\
\text { DIS- } \\
\text { SOLVED } \\
\text { (MG/L }\end{array}$ \\
\hline ATE & AS (A) & AS MG) & AS NA) & AS NA) & & AS $K$ ) & $\mathrm{HCO} 3)$ & AS $\mathrm{CO} 3$ ) & AS SO4) \\
\hline
\end{tabular}

AUG

\begin{tabular}{|c|c|c|c|c|c|c|c|c|c|}
\hline $03 \ldots$ & 25 & 5.5 & 18 & 23 & .9 & 5.2 & 52 & 0 & 43 \\
\hline $03 \ldots$ & -- & $\ldots$ & - & - & $\ldots$ & - & $\ldots$ & -- & -- \\
\hline $03 \ldots$ & -- & -- & - & -. & -- & -- & -- & -- & - \\
\hline $3 \ldots$ & 25 & 5.4 & 19 & 24 & .9 & 5.2 & 52 & 0 & 44 \\
\hline & $\begin{array}{l}\text { CHLO- } \\
\text { RIDE, } \\
\text { DIS- } \\
\text { SOLVED } \\
\text { (MG/L } \\
\text { AS CL) }\end{array}$ & $\begin{array}{l}\text { FLUO- } \\
\text { RIDE, } \\
\text { DIS- } \\
\text { SOLVED } \\
\text { (MG/L } \\
\text { AS F) }\end{array}$ & $\begin{array}{l}\text { SILICA, } \\
\text { DIS- } \\
\text { SOLVED } \\
\text { (MG/L } \\
\text { AS } \\
\text { SIO2) }\end{array}$ & $\begin{array}{c}\text { SOLIDS } \\
\text { SUM OF } \\
\text { CONSTI- } \\
\text { TUENTS, } \\
\text { DIS- } \\
\text { SOLVED }\end{array}$ & $\begin{array}{c}\text { NITRO- } \\
\text { GEN, } \\
\text { NO2+NO3 } \\
\text { TOTAL } \\
\text { (MG/L } \\
\text { AS N) }\end{array}$ & $\begin{array}{l}\text { NITRO- } \\
\text { GEN, } \\
\text { AMMONIA } \\
\text { TOTAL } \\
\text { (MG/L }\end{array}$ & $\begin{array}{c}\text { PHOS- } \\
\text { PHORUS, } \\
\text { TOTAL } \\
\text { (MG/L }\end{array}$ & $\begin{array}{l}\text { IRON, } \\
\text { DIS- } \\
\text { SOLVED } \\
\text { (UG/L }\end{array}$ & $\begin{array}{l}\text { MANGA- } \\
\text { NESE, } \\
\text { DIS- } \\
\text { SOLVED } \\
\text { (UG/L }\end{array}$ \\
\hline DATE & AS (L) & AS F) & SIO2) & $(M G / L)$ & AS N) & AS N) & AS P) & AS FE) & AS MN) \\
\hline
\end{tabular}

\begin{tabular}{|c|c|c|c|c|c|c|c|c|c|}
\hline \multicolumn{10}{|l|}{ AUG } \\
\hline $03 \ldots$ & 29 & .2 & 7.7 & 159 & .00 & .000 & .040 & 0 & 10 \\
\hline $03 \ldots$ & -- & -- & -- & -- & -- & -- & -- & -- & - \\
\hline $03 \ldots$ & -- & - & -- & -- & .00 & .010 & .040 & 0 & 10 \\
\hline $03 \ldots$ & 29 & -- & 8.2 & 162 & .00 & .010 & .040 & 0 & 180 \\
\hline
\end{tabular}

301940096315801 SOMERVILLE LAKE SITE AL

\begin{tabular}{|c|c|c|c|c|c|c|c|}
\hline & & & $\begin{array}{l}\text { SPE- } \\
\text { CIFIC } \\
\text { CON- }\end{array}$ & & & & $\begin{array}{c}\text { OXYGEN, } \\
\text { DIS- } \\
\text { SOIVED }\end{array}$ \\
\hline & & $\begin{array}{l}\text { SAMP- } \\
\text { LING }\end{array}$ & $\begin{array}{l}\text { DUCT- } \\
\text { ANCE }\end{array}$ & $\mathrm{PH}$ & $\begin{array}{l}\text { TEMPER- } \\
\text { ATURE, }\end{array}$ & $\begin{array}{c}\text { OXYGEN, } \\
\text { DIS- }\end{array}$ & $\begin{array}{l}\text { (PER- } \\
\text { CENT }\end{array}$ \\
\hline DATE & ГIME, & $\begin{array}{c}\text { DEPTH } \\
\text { (FT) }\end{array}$ & $\begin{array}{l}\text { (MICRO- } \\
\text { MHOS) }\end{array}$ & $\begin{array}{l}\text { FIELD } \\
\text { (UNITS) }\end{array}$ & $\begin{array}{c}\text { WATER } \\
\left(\begin{array}{ll}\text { DEG } & \text { C) }\end{array}\right.\end{array}$ & $\begin{array}{l}\text { SOLVED } \\
(M G / L)\end{array}$ & $\begin{array}{l}\text { SATUK- } \\
\text { ATION) }\end{array}$ \\
\hline
\end{tabular}

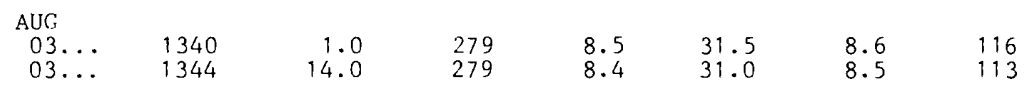

302026096341501 SOMERVILLE LAKE SITE BC

\begin{tabular}{|c|c|c|c|c|c|c|c|c|c|c|c|c|}
\hline DATE & TIME & $\begin{array}{r}\text { SAMP- } \\
\text { LING } \\
\text { DEPTH } \\
\text { (FT) }\end{array}$ & $\begin{array}{l}\text { SPE- } \\
\text { CIFIC } \\
\text { CON- } \\
\text { ACT- } \\
\text { ANCE } \\
\text { (MICRO- } \\
\text { MHOS) }\end{array}$ & $\begin{array}{c}\text { PH } \\
\text { FIELD } \\
\text { (UNITS) }\end{array}$ & 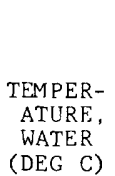 & $\begin{array}{c}\text { OXYGEN, } \\
\text { DIS- } \\
\text { SOLVED } \\
\text { (MG/L) }\end{array}$ & $\begin{array}{c}\text { OXYGEN, } \\
\text { DIS- } \\
\text { SOLVED } \\
\text { (PER- } \\
\text { CENT } \\
\text { SATUR- } \\
\text { ATION) }\end{array}$ & $\begin{array}{l}\text { NITRO- } \\
\text { GEN, } \\
\text { NO2+NO3 } \\
\text { TOTAL } \\
\text { (MG/L } \\
\text { AS N) }\end{array}$ & $\begin{array}{c}\text { NITRO- } \\
\text { GEN, } \\
\text { AMMONIA } \\
\text { TOTAL } \\
\text { (MG/L } \\
\text { AS N) }\end{array}$ & $\begin{array}{c}\text { PHOS- } \\
\text { PHORUS, } \\
\text { TOTAL } \\
\text { (MG/L } \\
\text { AS P) }\end{array}$ & $\begin{array}{l}\text { IKON, } \\
\text { DIS- } \\
\text { SULVED } \\
\text { (UG/L } \\
\text { AS FE) }\end{array}$ & $\begin{array}{l}\text { MANGA- } \\
\text { NESE, } \\
\text { DIS- } \\
\text { SOLVED } \\
\text { (UG/L } \\
\text { AS MN) }\end{array}$ \\
\hline
\end{tabular}

$\begin{array}{lrrrrrrrrrrrr}\text { AUG } & & & & & & & & \\ 03 \ldots & 1535 & 1.0 & 279 & 8.1 & 31.0 & 7.9 & 105 & .00 & .010 & .040 & 0 & 10 \\ 03 \ldots & 1539 & 14.0 & 279 & 7.5 & 30.0 & 6.0 & 79 & .04 & .010 & .050 & 10 & 10\end{array}$ 
Table 14--CHEMICAL-QUALITY SURVEYS OF SOMERVILLE LAKE AUGUST 3, 1979--Continued

301805096332501 SOMERVILLE LAKE SITE CC

\begin{tabular}{|c|c|c|c|c|c|c|c|c|c|c|c|c|}
\hline DATE & TIME & $\begin{array}{l}\text { SAMP- } \\
\text { LING } \\
\text { DEPTH } \\
(\mathrm{FT})\end{array}$ & $\begin{array}{l}\text { SPE- } \\
\text { CIFIC } \\
\text { CON- } \\
\text { DUCT- } \\
\text { ANCE } \\
\text { (MICRO- } \\
\text { MHOS) }\end{array}$ & $\begin{array}{c}\text { PH } \\
\text { FIELD } \\
\text { (UNITS) }\end{array}$ & $\begin{array}{c}\text { TEMPER- } \\
\text { ATURE, } \\
\text { WATER } \\
\text { (DEG C) }\end{array}$ & $\begin{array}{c}\text { OXYGEN, } \\
\text { DIS- } \\
\text { SOLVED } \\
(\mathrm{MG} / \mathrm{L})\end{array}$ & $\begin{array}{c}\text { OXYGEN, } \\
\text { DIS- } \\
\text { SOLVED } \\
\text { (PER- } \\
\text { CENT } \\
\text { SATUR- } \\
\text { ATION) }\end{array}$ & $\begin{array}{l}\text { NITRO- } \\
\text { GEN, } \\
\text { NO2+NO3 } \\
\text { TOTAL } \\
\text { (MG/L } \\
\text { AS N) }\end{array}$ & $\begin{array}{l}\text { NITRO- } \\
\text { GEN, } \\
\text { AMMONIA } \\
\text { TOTAL } \\
\text { (MG/L } \\
\text { AS N) }\end{array}$ & $\begin{array}{l}\text { PHOS- } \\
\text { PHORUS, } \\
\text { TOTAL } \\
\text { (MG/L } \\
\text { AS P) }\end{array}$ & $\begin{array}{l}\text { IRON, } \\
\text { DIS- } \\
\text { SOLVED } \\
\text { (UG/L } \\
\text { AS FE) }\end{array}$ & $\begin{array}{l}\text { MAINGA- } \\
\text { NESE, } \\
\text { DIS- } \\
\text { SOLVED } \\
\text { (UG/L } \\
\text { AS MN) }\end{array}$ \\
\hline
\end{tabular}

AUG

$03 \ldots . .1555$

1555
1559

11.0

279
279

2.9

$\begin{array}{ll}30.5 & 7.7 \\ 29.5 & 5.9\end{array}$

101
78

.00

$\begin{array}{ll}.010 & .040 \\ .010 & .040\end{array}$

0
0

301847096334601 SOMERVILLE LAKE SITE DR

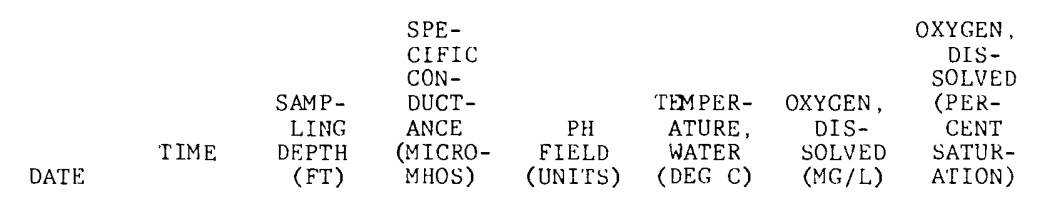

\begin{tabular}{|c|c|c|c|c|c|c|c|}
\hline \multicolumn{8}{|l|}{ AUG } \\
\hline $\begin{array}{l}03 \ldots \\
03 \ldots \\
03 \ldots \\
03 \ldots\end{array}$ & $\begin{array}{l}1430 \\
1434 \\
1438 \\
1442\end{array}$ & $\begin{array}{l}1.0 \\
10.0 \\
20.0 \\
24.0\end{array}$ & $\begin{array}{l}283 \\
283 \\
283 \\
283\end{array}$ & $\begin{array}{l}8.3 \\
8.0 \\
7.0 \\
6.7\end{array}$ & $\begin{array}{l}31.0 \\
30.0 \\
29.5 \\
29.0\end{array}$ & $\begin{array}{r}8.2 \\
7.6 \\
4.5 \\
.6\end{array}$ & $\begin{array}{r}109 \\
100 \\
59 \\
8\end{array}$ \\
\hline
\end{tabular}

301904096335601 SOMERVILLF LAKE SITE DC

\begin{tabular}{|c|c|c|c|c|c|c|c|c|c|}
\hline DATE & T IME, & $\begin{array}{l}\text { SAMP- } \\
\text { LING } \\
\text { DEPTH } \\
\text { (FT) }\end{array}$ & $\begin{array}{l}\text { SPE- } \\
\text { CIEIC } \\
\text { CON- } \\
\text { DUCT- } \\
\text { ANCE } \\
\text { (MICRO- } \\
\text { MHOS) }\end{array}$ & $\begin{array}{c}\text { PH } \\
\text { FIELD } \\
\text { (UNITS) }\end{array}$ & $\begin{array}{l}\text { TEMPER- } \\
\text { ATURE, } \\
\text { WATER } \\
\text { (DEG }\end{array}$ & $\begin{array}{c}\text { OXYGEN, } \\
\text { DIS- } \\
\text { SOLVED } \\
(\mathrm{MG} / \mathrm{L})\end{array}$ & $\begin{array}{c}\text { OXYGEN, } \\
\text { DIS- } \\
\text { SOLVED } \\
\text { (PER- } \\
\text { CENT } \\
\text { SATUR- } \\
\text { ATION) }\end{array}$ & $\begin{array}{l}\text { HARD- } \\
\text { NESS } \\
\text { (MG/L } \\
\text { AS } \\
\text { CACO3) }\end{array}$ & $\begin{array}{l}\text { HARD- } \\
\text { NESS } \\
\text { NONCAR- } \\
\text { BONATE } \\
\text { (MG/L } \\
\text { CACO3) }\end{array}$ \\
\hline $\begin{array}{l}\text { AUG } \\
03 \ldots \\
03 \ldots \\
03 \ldots \\
03 \ldots\end{array}$ & $\begin{array}{l}1410 \\
1414 \\
1418 \\
1422\end{array}$ & $\begin{array}{r}1.0 \\
10.0 \\
20.0 \\
28.0\end{array}$ & $\begin{array}{l}283 \\
283 \\
283 \\
283\end{array}$ & $\begin{array}{l}8.5 \\
7.9 \\
7.2 \\
6.9\end{array}$ & $\begin{array}{l}31.0 \\
30.0 \\
29.5 \\
29.5\end{array}$ & $\begin{array}{l}8.8 \\
7.1 \\
5.6 \\
2.8\end{array}$ & $\begin{array}{r}117 \\
93 \\
74 \\
37\end{array}$ & $\begin{array}{l}85 \\
-- \\
78\end{array}$ & $\begin{array}{l}41 \\
-- \\
34\end{array}$ \\
\hline DATE & $\begin{array}{l}\text { CALCIUM } \\
\text { DIS- } \\
\text { SOLVED } \\
\text { (MG/L } \\
\text { AS CA) }\end{array}$ & $\begin{array}{l}\text { MAGNE- } \\
\text { SIUM, } \\
\text { DIS- } \\
\text { SOLVED } \\
\text { (MG/L } \\
\text { AS MG) }\end{array}$ & $\begin{array}{l}\text { SODIUM, } \\
\text { DIS- } \\
\text { SOLVED } \\
\text { (MG/L } \\
\text { AS NA) }\end{array}$ & $\begin{array}{c}\text { SODIUM+ } \\
\text { POTAS- } \\
\text { SIUM } \\
\text { DIS- } \\
\text { SOLVED } \\
\text { (MG/L } \\
\text { AS NA) }\end{array}$ & $\begin{array}{c}\text { SODIUM } \\
\text { AD- } \\
\text { SORP- } \\
\text { TION } \\
\text { RATIO }\end{array}$ & $\begin{array}{l}\text { POTAS- } \\
\text { SIUM, } \\
\text { DIS- } \\
\text { SOLVED } \\
\text { (MG/L } \\
\text { AS K) }\end{array}$ & $\begin{array}{c}\text { BICAR- } \\
\text { BONATE } \\
\text { (MG / L } \\
\text { AS } \\
\text { HCO3) }\end{array}$ & $\begin{array}{c}\text { CAR- } \\
\text { BONATE } \\
\text { (MG/L } \\
\text { AS CO3) }\end{array}$ & $\begin{array}{l}\text { SULFATE } \\
\text { DIS- } \\
\text { SOLVED } \\
\text { (MG/L } \\
\text { AS SO4) }\end{array}$ \\
\hline
\end{tabular}

\begin{tabular}{|c|c|c|c|c|c|c|c|c|c|}
\hline \multicolumn{10}{|l|}{ AUG } \\
\hline $03 \ldots$ & 25 & 5.6 & 20 & 20 & .9 & 5.3 & 54 & 0 & 44 \\
\hline $03 \ldots$ & -- & -- & -- & -- & -- & -- & -- & -- & -- \\
\hline $03 \ldots$ & -- & -- & -- & -- & -- & -- & -- & -- & $\cdots$ \\
\hline $03 \ldots$ & 22 & 5.7 & 18 & 23 & .9 & 5.2 & 54 & 0 & 43 \\
\hline
\end{tabular}

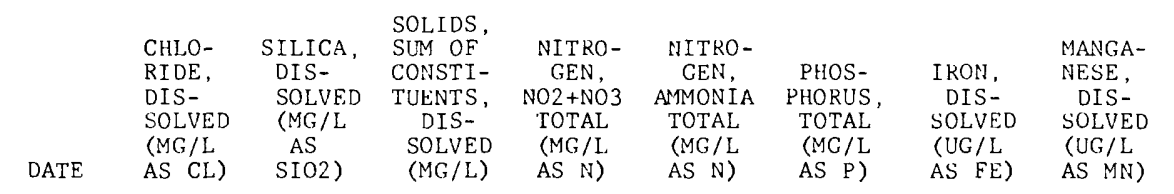

\begin{tabular}{|c|c|c|c|c|c|c|c|c|}
\hline \multicolumn{9}{|l|}{ AUG } \\
\hline $03 \ldots$ & 30 & 8.1 & 165 & .02 & .000 & .050 & 0 & 10 \\
\hline $03 \ldots$ & -- & -- & -- & -- & - & -- & -- & -- \\
\hline $03 \ldots$ & -- & - & $=-$ & .00 & .010 & .040 & 0 & 40 \\
\hline $03 \ldots$ & 29 & 8.5 & 158 & .00 & .030 & .050 & 0 & 200 \\
\hline
\end{tabular}


Table 14--CHEMICAL-QUALITY SURVEYS OF SOMERVILLE LAKF, AUGUST 3, 1979--Continued

301817096364101 SOMERVILLE. LAKE, SITE EC

\begin{tabular}{|c|c|c|c|c|c|c|c|}
\hline DA'TE & T IME & $\begin{array}{l}\text { SAMP- } \\
\text { LING } \\
\text { DEPTH } \\
\text { (FT) }\end{array}$ & $\begin{array}{l}\text { SPE- } \\
\text { CIFIC } \\
\text { CON- } \\
\text { DUCT- } \\
\text { ANCE } \\
\text { (MICRO- } \\
\text { MHOS) }\end{array}$ & $\begin{array}{c}\text { PH } \\
\text { FIELD } \\
\text { (UNITS) }\end{array}$ & $\begin{array}{l}\text { TEMPER- } \\
\text { ATURE, } \\
\text { WATER } \\
\text { (DEG }\end{array}$ & $\begin{array}{l}\text { OXYGEN, } \\
\text { DIS- } \\
\text { SOLVED } \\
(M G / L)\end{array}$ & $\begin{array}{l}\text { OXYGEN, } \\
\text { DIS- } \\
\text { SOLVED } \\
\text { (PER- } \\
\text { CENT } \\
\text { SATUR- } \\
\text { ATION) }\end{array}$ \\
\hline $\begin{array}{l}\text { AUG } \\
03 \ldots \\
03 \ldots \\
03 \ldots\end{array}$ & $\begin{array}{l}1520 \\
1524 \\
1528\end{array}$ & $\begin{array}{r}1.0 \\
10.0 \\
24.0\end{array}$ & $\begin{array}{l}284 \\
284 \\
284\end{array}$ & $\begin{array}{l}8.2 \\
7.4 \\
6.9\end{array}$ & $\begin{array}{l}30.5 \\
29.5 \\
29.5\end{array}$ & $\begin{array}{l}8.7 \\
6.5 \\
3.9\end{array}$ & $\begin{array}{r}115 \\
85 \\
51\end{array}$ \\
\hline
\end{tabular}

301754096380801 SOMERVILLE LAKE SITE FC

\begin{tabular}{|c|c|c|c|c|c|c|c|c|c|}
\hline DATE & TIME & $\begin{array}{l}\text { SAMP- } \\
\text { LING } \\
\text { DEPTH } \\
\text { (FT) }\end{array}$ & $\begin{array}{l}\text { SPE- } \\
\text { CIFIC } \\
\text { CON- } \\
\text { DUCT- } \\
\text { ANCE } \\
\text { (MICRO- } \\
\text { MHOS) }\end{array}$ & $\begin{array}{c}\text { PH } \\
\text { FIELD } \\
\text { (UNITS) }\end{array}$ & $\begin{array}{l}\text { TEMPER- } \\
\text { ATURE, } \\
\text { WATER } \\
\text { (DEG C) }\end{array}$ & $\begin{array}{c}\text { OXYGEN, } \\
\text { DIS- } \\
\text { SOLVED } \\
(\mathrm{MG} / \mathrm{L})\end{array}$ & $\begin{array}{c}\text { OXYGEN, } \\
\text { DIS- } \\
\text { SOLVED } \\
\text { (PER- } \\
\text { CENT } \\
\text { SATUR- } \\
\text { ATION) }\end{array}$ & $\begin{array}{l}\text { HARD- } \\
\text { NESS } \\
(\mathrm{NG} / \mathrm{L} \\
\text { AS } \\
\text { CACO3) }\end{array}$ & $\begin{array}{c}\text { HARD- } \\
\text { NESS, } \\
\text { NONCAR- } \\
\text { BONATE } \\
\text { (MG / L } \\
\text { CACO3) }\end{array}$ \\
\hline $\begin{array}{l}\text { AUG } \\
03 \ldots \\
03 \ldots\end{array}$ & $\begin{array}{l}1500 \\
1504\end{array}$ & $\begin{array}{r}1.0 \\
13.0\end{array}$ & $\begin{array}{l}322 \\
307\end{array}$ & $\begin{array}{l}8.1 \\
6.9\end{array}$ & $\begin{array}{l}31.5 \\
29.5\end{array}$ & $\begin{array}{l}8.7 \\
3.2\end{array}$ & $\begin{array}{r}118 \\
42\end{array}$ & $\begin{array}{l}99 \\
92\end{array}$ & $\begin{array}{l}52 \\
46\end{array}$ \\
\hline DATE & $\begin{array}{l}\text { CALCIUM } \\
\text { DIS- } \\
\text { SOLVED } \\
\text { (MG/L } \\
\text { AS CA) }\end{array}$ & $\begin{array}{l}\text { MAGNE- } \\
\text { SIUM, } \\
\text { DIS- } \\
\text { SOLVED } \\
\text { (MG/L } \\
\text { AS MG) }\end{array}$ & $\begin{array}{l}\text { SODIUM, } \\
\text { DIS- } \\
\text { SOLVED } \\
\text { (MG/L } \\
\text { AS NA) }\end{array}$ & $\begin{array}{c}\text { SODIUM+ } \\
\text { POTAS- } \\
\text { SIUM } \\
\text { DIS- } \\
\text { SOLVED } \\
\text { (MG/L } \\
\text { AS NA) }\end{array}$ & $\begin{array}{c}\text { SODIUM } \\
\text { AD- } \\
\text { SORP- } \\
\text { 'TION } \\
\text { RAT IO }\end{array}$ & $\begin{array}{l}\text { POIAS- } \\
\text { SIUM, } \\
\text { DIS- } \\
\text { SOLVED } \\
\text { (MG/L } \\
\text { AS K) }\end{array}$ & $\begin{array}{c}\text { BICAR- } \\
\text { BONATE } \\
\text { (MG/L } \\
\text { AS } \\
\text { HCO3) }\end{array}$ & $\begin{array}{c}\text { CAR- } \\
\text { BONA'IE } \\
(M G / L \\
\text { AS CO3) }\end{array}$ & $\begin{array}{l}\text { SULFATE } \\
\text { DIS- } \\
\text { SOLVLD } \\
\text { (:IG/L } \\
\text { AS SO4) }\end{array}$ \\
\hline
\end{tabular}

\begin{tabular}{|c|c|c|c|c|c|c|c|c|c|}
\hline AUG & & & & & & & & & \\
\hline $\begin{array}{l}03 \ldots \\
03 \ldots\end{array}$ & $\begin{array}{l}29 \\
27\end{array}$ & $\begin{array}{l}6.4 \\
6.0\end{array}$ & $\begin{array}{l}22 \\
21\end{array}$ & $\begin{array}{l}28 \\
26\end{array}$ & $\begin{array}{l}1.0 \\
1.0\end{array}$ & $\begin{array}{l}5.5 \\
5.3\end{array}$ & $\begin{array}{l}57 \\
56\end{array}$ & 0 & $\begin{array}{l}50 \\
47\end{array}$ \\
\hline
\end{tabular}

\begin{tabular}{|c|c|c|c|c|c|c|c|c|}
\hline & $\begin{array}{l}\text { CHLO- } \\
\text { RIDE, } \\
\text { DIS- } \\
\text { SOLVED } \\
\text { (MG/L }\end{array}$ & $\begin{array}{l}\text { SILICA, } \\
\text { DIS- } \\
\text { SOLVED } \\
\text { (MG/L } \\
\text { AS }\end{array}$ & $\begin{array}{l}\text { SOLIDS, } \\
\text { SUM OF } \\
\text { CONSTI- } \\
\text { TUENTS, } \\
\text { DIS- } \\
\text { SOLVED }\end{array}$ & $\begin{array}{l}\text { NITRO- } \\
\text { GEN } \\
\text { NO2+NO3 } \\
\text { TOTAL } \\
\text { (MG/L }\end{array}$ & $\begin{array}{l}\text { NITRO- } \\
\text { GEN, } \\
\text { AMMONIA } \\
\text { TOTAL } \\
\text { (MG/L }\end{array}$ & $\begin{array}{l}\text { PHOS- } \\
\text { PHORUS, } \\
\text { TOTAL } \\
\text { (MG/L }\end{array}$ & $\begin{array}{l}\text { IRON, } \\
\text { DIS- } \\
\text { SOLVED } \\
\text { (UG / L }\end{array}$ & $\begin{array}{l}\text { MANGA- } \\
\text { NESE, } \\
\text { DIS- } \\
\text { SOLVED } \\
\text { (UG/L }\end{array}$ \\
\hline DATE & AS CL) & SIO2) & $(M G / L)$ & AS N) & AS N) & & AS FE) & $A S$ MN) \\
\hline
\end{tabular}

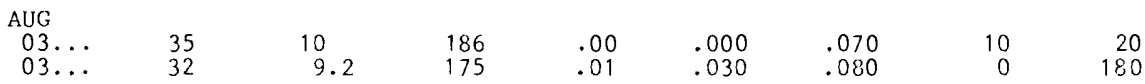


$\mathrm{FT}=$ feet, MICROMHOS = nicromhos per centimeter at $25^{\circ}$ Celsius; ${ }^{\circ} \mathrm{C}$ - degrees Celsius; $M G / L=$ milligrams per liter; UG/L = micrograms per liter

301908096313101 SOMERVILLE LAKE SITE AC

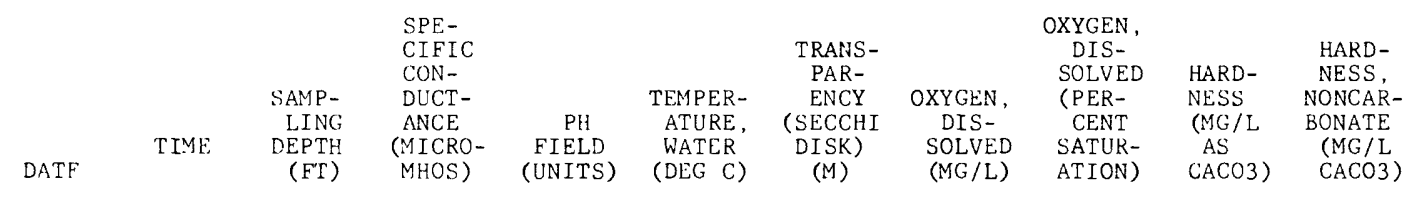

\begin{tabular}{|c|c|c|c|c|c|c|c|c|c|c|}
\hline \multicolumn{11}{|l|}{ JAN } \\
\hline $30 \ldots$ & 1320 & 1.0 & 318 & 7.6 & 11.0 & 1.00 & 10.0 & 91 & 90 & 44 \\
\hline $30 \ldots$ & 1322 & 10.0 & 318 & 7.6 & 11.0 & -- & 10.0 & 91 & -- & $=$ \\
\hline $30 \ldots$ & 1324 & 20.0 & 318 & 7.6 & 11.0 & -- & 9.9 & 90 & $=$ & $\cdots$ \\
\hline
\end{tabular}

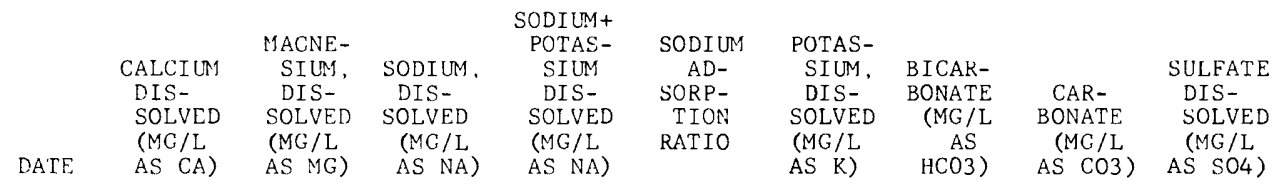

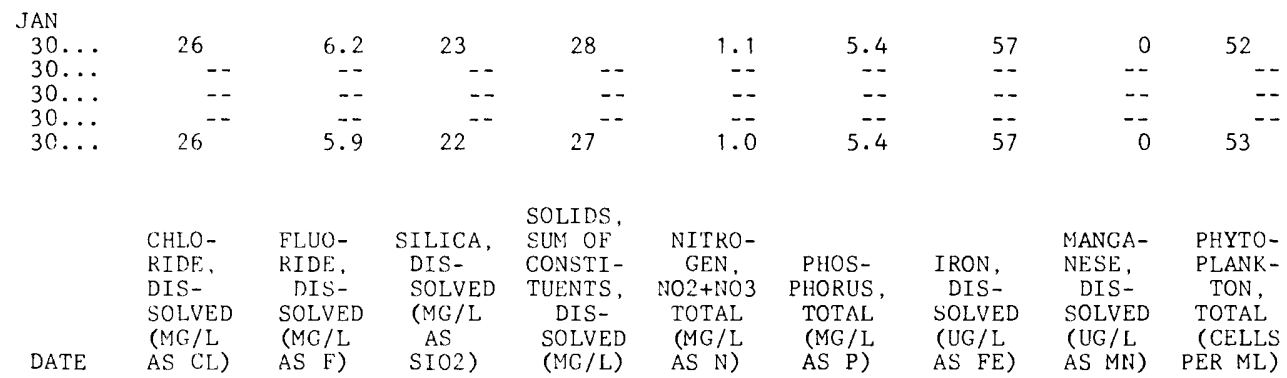

\begin{tabular}{|c|c|c|c|c|c|c|c|c|c|}
\hline JAN & & & & & & & & & \\
\hline $30 \ldots$ & 33 & .1 & 9.6 & 183 & .00 & .030 & $<10$ & $<1$ & -- \\
\hline $30 \ldots$ & -- & -- & -- & -- & - & - & -- & -. & 380000 \\
\hline $30 \ldots$ & -- & -- & -- & -- & .01 & .040 & 20 & 10 & -- \\
\hline $30 \ldots$ & - & - - & -- & - - & -. & - - & $-\cdots$ & -- & - \\
\hline $30 \ldots$ & 33 & - - & 9.5 & 183 & .01 & .040 & $<10$ & 5 & - \\
\hline
\end{tabular}

301940096315801 SOMERVILLE LAKE SITE AL

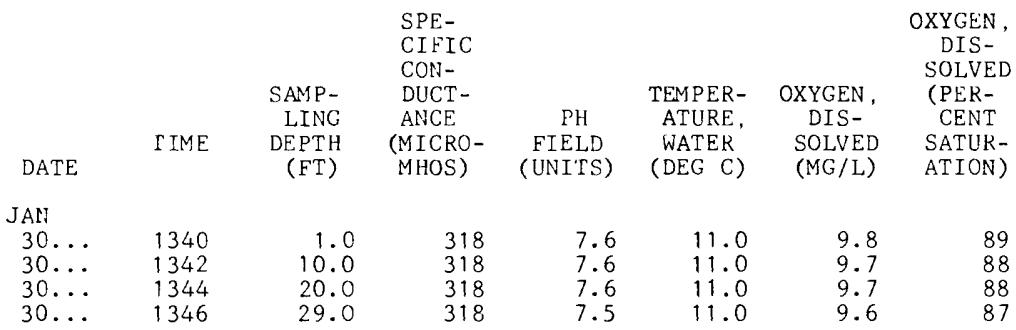


Table 15--CHEMICAL-QUALITY SURVEYS OF SOMERVILLE LAKE JANUARY 30, 1980--Continued

302026096341501 SOMERVILLE LAKE SITE BC

\begin{tabular}{|c|c|c|c|c|c|c|c|}
\hline DATE & TIME & $\begin{array}{l}\text { SAMP- } \\
\text { LING } \\
\text { DEPTH } \\
\text { (FT) }\end{array}$ & $\begin{array}{l}\text { SPE- } \\
\text { CIFIC } \\
\text { CON- } \\
\text { DUCT- } \\
\text { ANCE } \\
\text { (MICRO- } \\
\text { MHOS) }\end{array}$ & $\begin{array}{c}\text { PH } \\
\text { FIELD } \\
\text { (UNITS) }\end{array}$ & 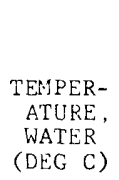 & $\begin{array}{c}\text { OXYGEN, } \\
\text { DIS- } \\
\text { SOLVED } \\
\text { (MG/L) }\end{array}$ & $\begin{array}{l}\text { OXYGEN, } \\
\text { DIS- } \\
\text { SOLVED } \\
\text { (PER- } \\
\text { CENT } \\
\text { SATUR- } \\
\text { ATION) }\end{array}$ \\
\hline IAN & & & & & & & \\
\hline $\begin{array}{l}30 \ldots \\
30 \ldots \\
30 \ldots\end{array}$ & $\begin{array}{l}1400 \\
1402 \\
1404\end{array}$ & $\begin{array}{r}1.0 \\
10.0 \\
15.0\end{array}$ & $\begin{array}{l}306 \\
306 \\
306\end{array}$ & $\begin{array}{l}7.6 \\
7.6 \\
7.5\end{array}$ & $\begin{array}{l}10.5 \\
10.5 \\
10.5\end{array}$ & $\begin{array}{l}9.9 \\
9.9 \\
9.7\end{array}$ & $\begin{array}{l}89 \\
89 \\
87\end{array}$ \\
\hline
\end{tabular}

301805096332501 SOMERVILLE LAKE SITE CC

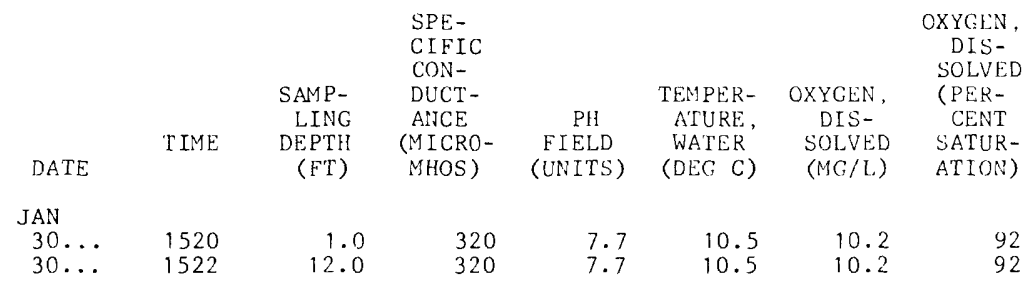

301847096334601 SOMERVILLE LAKE SI'TE DR

\begin{tabular}{|c|c|c|c|c|c|c|c|}
\hline DATE & TIME & $\begin{array}{l}\text { SAMP- } \\
\text { LING } \\
\text { DEPTH } \\
\text { (FT) }\end{array}$ & $\begin{array}{l}\text { SPE- } \\
\text { CIFIC } \\
\text { CON- } \\
\text { DUCT- } \\
\text { ANCE } \\
\text { (MICRO- } \\
\text { MHOS) }\end{array}$ & $\begin{array}{c}\text { PH } \\
\text { FILLD } \\
\text { (UNITS) }\end{array}$ & $\begin{array}{c}\text { TEMPER- } \\
\text { ATURC, } \\
\text { WATER } \\
\text { (DEG C) }\end{array}$ & $\begin{array}{c}\text { OXYGEN, } \\
\text { DIS- } \\
\text { SOLVED } \\
\text { (MG/L) }\end{array}$ & $\begin{array}{c}\text { OXYGEN, } \\
\text { DIS- } \\
\text { SOLVED } \\
\text { (PER- } \\
\text { CENT } \\
\text { SATUR- } \\
\text { ATION) }\end{array}$ \\
\hline \multicolumn{8}{|l|}{ JAN } \\
\hline $\begin{array}{l}30 \ldots \\
30 \ldots \\
30 \ldots\end{array}$ & $\begin{array}{l}1420 \\
1422 \\
1424\end{array}$ & $\begin{array}{r}1.0 \\
10.0 \\
24.0\end{array}$ & $\begin{array}{l}321 \\
321 \\
321\end{array}$ & $\begin{array}{l}7.6 \\
7.5 \\
7.5\end{array}$ & $\begin{array}{l}11.0 \\
11.0 \\
11.0\end{array}$ & $\begin{array}{l}9.6 \\
9.5 \\
9.5\end{array}$ & $\begin{array}{l}87 \\
86 \\
86\end{array}$ \\
\hline
\end{tabular}

301904096335601 SOMERVILLE LAKE SITE DC

\begin{tabular}{|c|c|c|c|c|c|c|c|c|c|}
\hline DATE & TIME & $\begin{array}{l}\text { SAMP- } \\
\text { LING } \\
\text { DFPTH } \\
\text { (FT) }\end{array}$ & $\begin{array}{l}\text { SPE- } \\
\text { CIFIC } \\
\text { CON- } \\
\text { DUCT- } \\
\text { ANCE } \\
\text { (MICRO- } \\
\text { MHOS) }\end{array}$ & $\begin{array}{c}\text { PH } \\
\text { FIELD } \\
\text { (UNITS) }\end{array}$ & 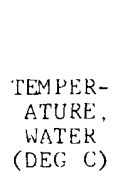 & $\begin{array}{l}\text { TRANS- } \\
\text { PAR- } \\
\text { ENCY } \\
\text { (SECCHI } \\
\text { DISK) } \\
\text { (M) }\end{array}$ & $\begin{array}{c}\text { OXYGEN, } \\
\text { DIS- } \\
\text { SOLVED } \\
\text { (MG/L) }\end{array}$ & $\begin{array}{c}\text { OXYCLN, } \\
\text { DLS- } \\
\text { SOI,VEI) } \\
\text { (PER- } \\
\text { CENT } \\
\text { SATUK- } \\
\text { A'TION) }\end{array}$ & $\begin{array}{l}\text { HARD - } \\
\text { NESS } \\
\text { (MG/L } \\
\text { AS } \\
\mathrm{CACO} 3)\end{array}$ \\
\hline $\begin{array}{l}\text { JAN } \\
30 \ldots \\
30 \ldots \\
30 \ldots \\
30 \ldots\end{array}$ & $\begin{array}{l}1410 \\
1412 \\
1414 \\
1416\end{array}$ & $\begin{array}{l}1.0 \\
10.0 \\
20.0 \\
25.0\end{array}$ & $\begin{array}{l}328 \\
328 \\
328 \\
328\end{array}$ & $\begin{array}{l}7.6 \\
7.5 \\
7.5 \\
7.5\end{array}$ & $\begin{array}{l}11.0 \\
11.0 \\
11.0 \\
11.0\end{array}$ & $\begin{array}{l}.70 \\
-- \\
-- \\
--\end{array}$ & $\begin{array}{l}9.7 \\
9.6 \\
9.6 \\
9.5\end{array}$ & $\begin{array}{l}88 \\
87 \\
87 \\
86\end{array}$ & $\begin{array}{l}93 \\
-. \\
93\end{array}$ \\
\hline & $\begin{array}{l}\text { HARD- } \\
\text { NESS, } \\
\text { NONCAR- } \\
\text { BONATE } \\
\text { (MG/L } \\
\text { CACO3) }\end{array}$ & $\begin{array}{l}\text { CALCIUM } \\
\text { DIS- } \\
\text { SOLVED } \\
\text { (MG/L } \\
\text { AS CA) }\end{array}$ & $\begin{array}{l}\text { MAGNE- } \\
\text { SIUM, } \\
\text { DIS- } \\
\text { SOLVED } \\
\text { (MG/L } \\
\text { AS MG) }\end{array}$ & $\begin{array}{l}\text { SODIUM, } \\
\text { DIS- } \\
\text { SOLVED } \\
\text { (MG/L } \\
\text { AS NA) }\end{array}$ & $\begin{array}{c}\text { SODIUM+ } \\
\text { POTAS- } \\
\text { SIUM } \\
\text { DIS- } \\
\text { SOLVED } \\
\text { (MG/I. } \\
\text { AS MA) }\end{array}$ & $\begin{array}{c}\text { SODIUM } \\
\text { AD- } \\
\text { SOKP- } \\
\text { TION } \\
\text { RATIO }\end{array}$ & $\begin{array}{l}\text { POTAS- } \\
\text { SIUM, } \\
\text { DIS- } \\
\text { SOLVED } \\
(M G / L \\
\text { AS K) }\end{array}$ & $\begin{array}{c}\text { BLCAR- } \\
\text { BONATE } \\
\text { (MG/L } \\
\text { AS } \\
\text { HCO3) }\end{array}$ & $\begin{array}{c}\text { CAR- } \\
\text { BONATE } \\
\text { (MG/L } \\
\text { AS CO3) }\end{array}$ \\
\hline
\end{tabular}

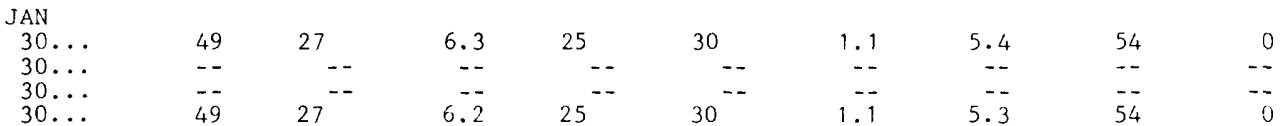

$-81-$ 
Table 15--CHEMICAL-QUALITY SURVEYS OF SOMERVILLE LAKE JANUARY 30, 1980--Continued

301904096335601 SOMERVILLE LAKE SITE DC--Continued

\begin{tabular}{|c|c|c|c|c|c|c|c|c|}
\hline DATE, & $\begin{array}{l}\text { SULFATE } \\
\text { DIS- } \\
\text { SOLVED } \\
\text { (MG/L } \\
\text { AS SO4) }\end{array}$ & $\begin{array}{l}\text { CHLO- } \\
\text { RIDE, } \\
\text { DIS- } \\
\text { SOLVED } \\
\text { (MG/L } \\
\text { AS CL) }\end{array}$ & $\begin{array}{l}\text { SILICA, } \\
\text { DIS- } \\
\text { SOLVED } \\
\text { (MG/L } \\
\text { AS } \\
\text { SIO2) }\end{array}$ & $\begin{array}{c}\text { SOLIDS, } \\
\text { SUM OF } \\
\text { CONSTI- } \\
\text { TUENTS, } \\
\text { DIS- } \\
\text { SOLVED } \\
\text { (MG/L) }\end{array}$ & $\begin{array}{c}\text { NITRO- } \\
\text { GEN } \\
\text { NO2+NO3 } \\
\text { TOTAL } \\
\text { (MG/L } \\
\text { AS N) }\end{array}$ & $\begin{array}{l}\text { PHOS- } \\
\text { PHORUS, } \\
\text { TOTAL } \\
\text { (MG/L } \\
\text { AS P) }\end{array}$ & $\begin{array}{l}\text { IRON, } \\
\text { DIS- } \\
\text { SOLVED } \\
\text { (UG / L } \\
\text { AS FE) }\end{array}$ & $\begin{array}{l}\text { MANGA- } \\
\text { NESE, } \\
\text { DIS- } \\
\text { SOLVED } \\
\text { (UG/L } \\
\text { AS MN) }\end{array}$ \\
\hline \multicolumn{9}{|l|}{ JAN } \\
\hline $\begin{array}{l}30 \ldots \\
30 \ldots\end{array}$ & $57 \ldots$ & 35 & $\begin{array}{r}9.8 \\
--\end{array}$ & $\begin{array}{c}192 \\
--\end{array}$ & $\begin{array}{l}.00 \\
.01\end{array}$ & $\begin{array}{l}.050 \\
.050\end{array}$ & $\begin{array}{l}20 \\
40\end{array}$ & $\begin{array}{r}1 \\
10\end{array}$ \\
\hline $30 \ldots$ & -- & -- & -- & $=-$ & -- & $=-$ & - & -1 \\
\hline $30 \ldots$ & 55 & 35 & 9.8 & 190 & .01 & .070 & 20 & 10 \\
\hline
\end{tabular}

$301 \varepsilon 17096364101$ SOMERVILLE LAKE SITE EC

\begin{tabular}{|c|c|c|c|c|c|c|c|}
\hline DATE & IIME & $\begin{array}{l}\text { SAMP- } \\
\text { LING } \\
\text { DEPTH } \\
\text { (FT) }\end{array}$ & $\begin{array}{l}\text { SPE- } \\
\text { CIEIC } \\
\text { CON- } \\
\text { DUCT- } \\
\text { ANCE } \\
\text { (MICRO- } \\
\text { MHOS) }\end{array}$ & $\begin{array}{c}\text { PH } \\
\text { FIELD } \\
\text { (UNITS) }\end{array}$ & 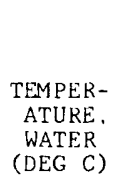 & $\begin{array}{c}\text { OXYGEN, } \\
\text { DIS- } \\
\text { SOLVED } \\
(M G / L)\end{array}$ & $\begin{array}{c}\text { OXYGEN, } \\
\text { DIS- } \\
\text { SOLVED } \\
\text { (PER- } \\
\text { CENT } \\
\text { SATUR- } \\
\text { ATION) }\end{array}$ \\
\hline \multicolumn{8}{|l|}{ JAN } \\
\hline $\begin{array}{l}30 \ldots \\
30 \ldots \\
30 \ldots\end{array}$ & $\begin{array}{l}1440 \\
1442 \\
1444\end{array}$ & $\begin{array}{r}1.0 \\
10.0 \\
17.0\end{array}$ & $\begin{array}{l}323 \\
323 \\
323\end{array}$ & $\begin{array}{l}7.4 \\
7.4 \\
7.3\end{array}$ & $\begin{array}{l}10.5 \\
10.5 \\
10.5\end{array}$ & $\begin{array}{l}9.4 \\
9.4 \\
9.3\end{array}$ & $\begin{array}{l}85 \\
85 \\
84\end{array}$ \\
\hline
\end{tabular}

301754096380801 SOMERVILLE LAKE SITE FC

\begin{tabular}{|c|c|c|c|c|c|c|c|c|c|}
\hline DATE & TIME & $\begin{array}{l}\text { SAMP- } \\
\text { LING } \\
\text { DEPTH } \\
(\mathrm{FT})\end{array}$ & $\begin{array}{l}\text { SPE- } \\
\text { CIFIC } \\
\text { CON- } \\
\text { DUCT- } \\
\text { ANCE } \\
\text { (MICRO- } \\
\text { MHOS) }\end{array}$ & $\begin{array}{c}\text { PH } \\
\text { FIELD } \\
\text { (UNITS) }\end{array}$ & 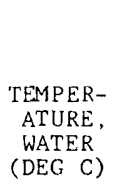 & $\begin{array}{c}\text { TRANS- } \\
\text { PAR- } \\
\text { ENCY } \\
\text { (SECCHI } \\
\text { DISK) } \\
\text { (M) }\end{array}$ & $\begin{array}{c}\text { OXYGEN, } \\
\text { DIS- } \\
\text { SOLVED } \\
\text { (MG /L) }\end{array}$ & $\begin{array}{c}\text { OXYGEN, } \\
\text { DIS- } \\
\text { SOLVED } \\
\text { (PER- } \\
\text { CENT } \\
\text { SATUR- } \\
\text { ATION) }\end{array}$ & $\begin{array}{l}\text { HARD- } \\
\text { NESS } \\
(\mathrm{MG} / \mathrm{L} \\
\mathrm{AS} \\
\mathrm{CACO} 3)\end{array}$ \\
\hline $\begin{array}{l}\text { JAN } \\
30 \ldots \\
30 \ldots \\
30 \ldots\end{array}$ & $\begin{array}{l}1455 \\
1456 \\
1457\end{array}$ & $\begin{array}{r}1.0 \\
.3 \\
14.0\end{array}$ & $\begin{array}{r}283 \\
--7 \\
317\end{array}$ & $\begin{array}{l}7.0 \\
-- \\
6.9\end{array}$ & $\begin{array}{r}10.0 \\
-- \\
9.5\end{array}$ & $\begin{array}{l}.20 \\
-- \\
--\end{array}$ & $\begin{array}{r}8.6 \\
-.5 \\
8.5\end{array}$ & $\begin{array}{l}77 \\
-- \\
75\end{array}$ & $\begin{array}{l}78 \\
-\overline{85}\end{array}$ \\
\hline DATE & $\begin{array}{l}\text { HARD- } \\
\text { NESS } \\
\text { NONCAR- } \\
\text { BONATE. } \\
\text { (MG/L } \\
\text { CACO3) }\end{array}$ & $\begin{array}{l}\text { CALCIUM } \\
\text { DIS- } \\
\text { SOLVED } \\
\text { (MG/L } \\
\text { AS CA) }\end{array}$ & $\begin{array}{l}\text { MAGNE- } \\
\text { SIUM. } \\
\text { DIS- } \\
\text { SOLVED } \\
\text { (MG/L } \\
\text { AS MC) }\end{array}$ & $\begin{array}{l}\text { SODIUM, } \\
\text { DIS- } \\
\text { SOLVED } \\
\text { (MG/L } \\
\text { AS NA) }\end{array}$ & $\begin{array}{c}\text { SODIUM+ } \\
\text { POTAS- } \\
\text { SIUM } \\
\text { DIS- } \\
\text { SOLVED } \\
\text { (MG/L } \\
\text { AS NA) }\end{array}$ & $\begin{array}{c}\text { SODIUM } \\
\text { AD- } \\
\text { SORP- } \\
\text { TION } \\
\text { RATIO }\end{array}$ & $\begin{array}{l}\text { POTAS- } \\
\text { SIUM, } \\
\text { DIS- } \\
\text { SOLVED } \\
(M G / L \\
\text { AS K) }\end{array}$ & $\begin{array}{c}\text { BICAR- } \\
\text { BONATE } \\
\text { (MG / L } \\
\text { AS } \\
\text { HCO3) }\end{array}$ & $\begin{array}{c}\text { CAR- } \\
\text { BONATE } \\
(\mathrm{MG} / \mathrm{L} \\
\mathrm{AS} \mathrm{CO} 3)\end{array}$ \\
\hline
\end{tabular}
JAN

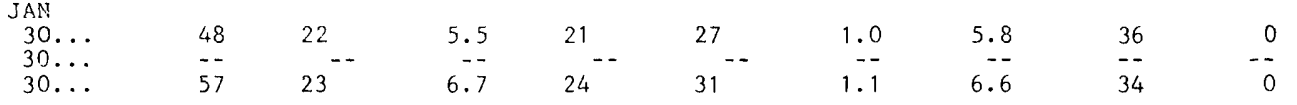

\begin{tabular}{|c|c|c|c|c|c|c|c|c|c|}
\hline & $\begin{array}{l}\text { SULFATE } \\
\text { DIS- } \\
\text { SOLVED } \\
\text { (MG / L }\end{array}$ & $\begin{array}{l}\text { CHLO- } \\
\text { RIDE, } \\
\text { DIS- } \\
\text { SOLVED } \\
\text { (MG/L }\end{array}$ & $\begin{array}{l}\text { SILICA, } \\
\text { DIS- } \\
\text { SOLVED } \\
\text { (MG/L } \\
\text { AS }\end{array}$ & $\begin{array}{l}\text { SOLIDS, } \\
\text { SUM OF } \\
\text { CONSTI- } \\
\text { TUENTS, } \\
\text { DIS- } \\
\text { SOLVED }\end{array}$ & $\begin{array}{l}\text { NITRO- } \\
\text { GEN, } \\
\text { NO2+NO3 } \\
\text { TOTAL } \\
\text { (MG/L }\end{array}$ & $\begin{array}{l}\text { PHOS- } \\
\text { PHORUS, } \\
\text { TOTAL } \\
\text { (MG/L }\end{array}$ & $\begin{array}{l}\text { IRON, } \\
\text { DIS- } \\
\text { SOLVED } \\
\text { (UG/L }\end{array}$ & $\begin{array}{l}\text { MANGA- } \\
\text { NESE, } \\
\text { DIS- } \\
\text { SOLVED } \\
\text { (UG/L }\end{array}$ & $\begin{array}{l}\text { PHYTO- } \\
\text { PLANK- } \\
\text { TON , } \\
\text { TOTAL } \\
\text { (CELLS }\end{array}$ \\
\hline $\mathrm{ATE}$ & AS S04) & AS CL) & SIO2) & $(M G / L)$ & AS N) & AS P) & AS FE) & AS $M N$ ) & PER ML) \\
\hline
\end{tabular}

\begin{tabular}{|c|c|c|c|c|c|c|c|c|c|}
\hline JAN & & & & & & & & & \\
\hline $\begin{array}{l}30 \ldots \\
30 \ldots\end{array}$ & & 33 & 11 & $\begin{array}{r}173 \\
--\end{array}$ & .11 & .120 & $\begin{array}{r}180 \\
--\end{array}$ & $\begin{array}{l}40 \\
--\end{array}$ & $970 \overline{0}$ \\
\hline $30 \ldots$ & 66 & 33 & 13 & 190 & .08 & .150 & 350 & 80 & - \\
\hline
\end{tabular}


$\mathrm{FT}=$ feet $;$ MICROMHOS = micromhos per centimeter at $25^{\circ} \mathrm{Celsius;}{ }^{\circ} \mathrm{C}$ - degrees Celsius; $M G / L=$ milligrams per liter; UG/L = micrograms per liter

301908096313101 SOMERVILLE LAKE SI'TE AC

\begin{tabular}{|c|c|c|c|c|c|c|c|c|c|}
\hline DATE & TIME & $\begin{array}{c}\text { SAMP- } \\
\text { LING } \\
\text { DEPTH } \\
\text { (FT) }\end{array}$ & $\begin{array}{l}\text { SPE- } \\
\text { CIFIC } \\
\text { CON- } \\
\text { DUCT- } \\
\text { ANCE } \\
\text { (MICRO- } \\
\text { MHOS) }\end{array}$ & $\begin{array}{c}\text { PH } \\
\text { FIELD } \\
\text { (UNITS) }\end{array}$ & $\begin{array}{c}\text { TEMPER- } \\
\text { ATURE, } \\
\text { WATER } \\
\text { (DEG C) }\end{array}$ & $\begin{array}{c}\text { TRANS - } \\
\text { PAR- } \\
\text { ENCY } \\
\text { (SECCHI } \\
\text { DISK) } \\
(M)\end{array}$ & $\begin{array}{c}\text { OXYGEN, } \\
\text { DIS- } \\
\text { SOLVED } \\
\text { (MG/L) }\end{array}$ & $\begin{array}{c}\text { OXYGEN, } \\
\text { DIS- } \\
\text { SOLVED } \\
\text { (PER- } \\
\text { CENT } \\
\text { SATUR- } \\
\text { ATION) }\end{array}$ & $\begin{array}{l}\text { HARD- } \\
\text { NESS } \\
\text { (MG/L } \\
\text { AS } \\
\text { CACO3) }\end{array}$ \\
\hline $\begin{array}{r}\text { MAY } \\
21 \ldots \\
21 \ldots \\
21 \ldots \\
21 \ldots \\
21 \ldots\end{array}$ & $\begin{array}{l}1122 \\
1123 \\
1124 \\
1126 \\
1128\end{array}$ & $\begin{array}{r}1.0 \\
1.5 \\
10.0 \\
20.0 \\
30.0\end{array}$ & $\begin{array}{r}364 \\
-- \\
364 \\
364 \\
362\end{array}$ & $\begin{array}{l}7.9 \\
-- \\
7.4 \\
7.0 \\
6.8\end{array}$ & $\begin{array}{r}24.0 \\
.- \\
23.0 \\
23.0 \\
22.5\end{array}$ & $\begin{array}{c}91 \\
-- \\
-- \\
-- \\
--\end{array}$ & $\begin{array}{r}8.2 \\
-- \\
7.7 \\
6.4 \\
3.8\end{array}$ & $\begin{array}{l}98 \\
-- \\
90 \\
74 \\
44\end{array}$ & $\begin{array}{r}110 \\
=- \\
-\overline{-} \\
110\end{array}$ \\
\hline DATE & $\begin{array}{l}\text { HARD- } \\
\text { NESS } \\
\text { NONCAR- } \\
\text { BONATE } \\
\text { (MG/L } \\
\text { CACO3) }\end{array}$ & $\begin{array}{l}\text { CALCIUM } \\
\text { DIS- } \\
\text { SOLVED } \\
\text { (MG/L } \\
\text { AS CA) }\end{array}$ & $\begin{array}{l}\text { MAGNE- } \\
\text { SIUM, } \\
\text { DIS- } \\
\text { SOLVED } \\
\text { (MG/L } \\
\text { AS MG) }\end{array}$ & $\begin{array}{l}\text { SODIUM, } \\
\text { DIS- } \\
\text { SOLVED } \\
\text { (MG/L } \\
\text { AS NA) }\end{array}$ & $\begin{array}{c}\text { SODIUM } \\
\text { AD- } \\
\text { SORP- } \\
\text { TION } \\
\text { RATIO }\end{array}$ & $\begin{array}{l}\text { POTAS- } \\
\text { SIUM, } \\
\text { DIS- } \\
\text { SOLVED } \\
\text { (MG/L } \\
\text { AS K) }\end{array}$ & $\begin{array}{c}\text { BICAR- } \\
\text { BONATE } \\
\text { (MG/L } \\
\text { AS } \\
\text { HCO3) }\end{array}$ & $\begin{array}{c}\text { CAR- } \\
\text { BONATE } \\
\text { (MG/L } \\
\text { AS CO3) }\end{array}$ & $\begin{array}{l}\text { SULEATE } \\
\text { DIS- } \\
\text { SOLVED } \\
\text { (MG/L } \\
\text { AS SO4) }\end{array}$ \\
\hline $\begin{array}{r}\text { MAY } \\
21 \ldots \\
21 \ldots \\
21 \ldots \\
21 \ldots \\
21 \ldots\end{array}$ & $\begin{array}{l}62 \\
-- \\
-- \\
62\end{array}$ & ${ }_{30}^{30}$ & $\begin{array}{l}7.3 \\
-- \\
-- \\
-- \\
7.3\end{array}$ & $25^{--}$ & $\begin{array}{r}1.1 \\
-- \\
-- \\
-- \\
1.1\end{array}$ & $\begin{array}{r}5.7 \\
-- \\
-- \\
-- \\
5.8\end{array}$ & $\begin{array}{l}52 \\
-- \\
-- \\
52\end{array}$ & $\begin{array}{c}0 \\
-- \\
-- \\
0\end{array}$ & $\begin{array}{r}64 \\
62^{--}\end{array}$ \\
\hline DATE & $\begin{array}{l}\text { CHLO- } \\
\text { RIDE, } \\
\text { DIS- } \\
\text { SOLVED } \\
\text { (MG/L } \\
\text { AS CL) }\end{array}$ & $\begin{array}{l}\text { FLUO- } \\
\text { RIDE, } \\
\text { DIS- } \\
\text { SOLVED } \\
\text { (MG/L } \\
\text { AS F) }\end{array}$ & $\begin{array}{l}\text { SILICA, } \\
\text { DIS- } \\
\text { SOLVED } \\
\text { (MG/L } \\
\text { AS } \\
\text { SIO2) }\end{array}$ & $\begin{array}{l}\text { SOLIDS, } \\
\text { SUM OF } \\
\text { CONSTI- } \\
\text { TUENTS, } \\
\text { DIS- } \\
\text { SOLVED } \\
\text { (MG/L) }\end{array}$ & $\begin{array}{l}\text { NITRO- } \\
\text { GEN. } \\
\text { NO2+NO3 } \\
\text { TOTAL } \\
\text { (MG/L } \\
\text { AS N) }\end{array}$ & $\begin{array}{c}\text { PHOS- } \\
\text { PHORUS, } \\
\text { TOTAL } \\
\text { (MG/L } \\
\text { AS P) }\end{array}$ & $\begin{array}{l}\text { IRON, } \\
\text { DIS- } \\
\text { SOLVED } \\
\text { (UG/L } \\
\text { AS FE) }\end{array}$ & $\begin{array}{l}\text { NANGA- } \\
\text { NESE, } \\
\text { DIS- } \\
\text { SOLVED } \\
\text { (UG/L } \\
\text { AS MN) }\end{array}$ & $\begin{array}{l}\text { PHYTO- } \\
\text { PLANK- } \\
\text { TON, } \\
\text { TOTAL } \\
\text { (CELLS } \\
\text { PER ML) }\end{array}$ \\
\hline
\end{tabular}

MAY

\begin{tabular}{|c|c|c|c|c|c|c|c|c|c|}
\hline $21 \ldots$ & 38 & .1 & 7.1 & 203 & .03 & .030 & $<10$ & 4 & - \\
\hline $21 \ldots$ & -- & - & - & -- & -- & $\cdots$ & -- & - & 52000 \\
\hline $1 \ldots$ & -- & - & - & -- & .02 & .040 & 40 & 20 & -- \\
\hline $1 \ldots$ & -- & - & -- & -- & - & -- & -- & -- & -- \\
\hline$\ldots$ & 38 & -- & 8.2 & 203 & .06 & .040 & $<10$ & 140 & 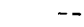 \\
\hline
\end{tabular}

301940096315801 SOMERVILLE LAKE SITE AL

\begin{tabular}{|c|c|c|c|c|c|c|c|}
\hline & & & $\begin{array}{l}\text { SPE- } \\
\text { CIFIC } \\
\text { CON- }\end{array}$ & & & & $\begin{array}{c}\text { OXYGEN, } \\
\text { DIS- } \\
\text { SOLVED }\end{array}$ \\
\hline & & $\begin{array}{l}\text { SAMP- } \\
\text { LING }\end{array}$ & $\begin{array}{l}\text { DUCT- } \\
\text { ANCE }\end{array}$ & $\mathrm{PH}$ & $\begin{array}{l}\text { 'TEMPER- } \\
\text { ATURE, }\end{array}$ & $\begin{array}{c}\text { OXYGEN, } \\
\text { DIS- }\end{array}$ & $\begin{array}{c}\text { (PER- } \\
\text { CENT }\end{array}$ \\
\hline DATE & TIME & $\begin{array}{c}\text { DEPTH } \\
\text { (FT) }\end{array}$ & $\begin{array}{l}\text { (MICRO- } \\
\text { MHOS) }\end{array}$ & $\begin{array}{l}\text { FIELD } \\
\text { (UNITS) }\end{array}$ & $\begin{array}{l}\text { WATER } \\
\left(\begin{array}{ll}\text { DEG } & \text { C) }\end{array}\right.\end{array}$ & $\begin{array}{l}\text { SOLVLD } \\
(\mathrm{MG} / \mathrm{L})\end{array}$ & $\begin{array}{l}\text { SATUR- } \\
\text { A'TION ) }\end{array}$ \\
\hline
\end{tabular}

\begin{tabular}{|c|c|c|c|c|c|c|c|}
\hline \multicolumn{8}{|l|}{ MAY } \\
\hline $\begin{array}{l}21 \ldots \\
21 \ldots\end{array}$ & $\begin{array}{l}1149 \\
1151\end{array}$ & $\begin{array}{r}1.0 \\
10.0\end{array}$ & $\begin{array}{l}364 \\
364\end{array}$ & $\begin{array}{l}7.9 \\
7.5\end{array}$ & $\begin{array}{l}24.5 \\
23.5\end{array}$ & $\begin{array}{l}8.6 \\
7.9\end{array}$ & $\begin{array}{r}102 \\
93\end{array}$ \\
\hline $21 \ldots$ & 1153 & 22.0 & 364 & 6.9 & 23.0 & 5.6 & 65 \\
\hline
\end{tabular}

302026096341501 SOMERVILLE LAKE SITE BC

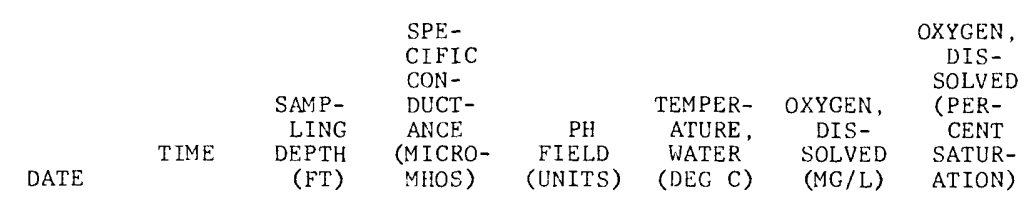

\begin{tabular}{|c|c|c|c|c|c|c|c|}
\hline & & & & & & & \\
\hline $\begin{array}{l}21 \ldots \\
21 \ldots \\
21 \ldots\end{array}$ & $\begin{array}{l}1204 \\
1206 \\
1208\end{array}$ & $\begin{array}{r}1.0 \\
10.0 \\
15.0\end{array}$ & $\begin{array}{l}347 \\
357 \\
340\end{array}$ & $\begin{array}{l}8.3 \\
8.1 \\
6.8\end{array}$ & $\begin{array}{l}26.0 \\
24.5 \\
23.5\end{array}$ & $\begin{array}{l}9.6 \\
9.0 \\
5.1\end{array}$ & $\begin{array}{r}119 \\
107 \\
60\end{array}$ \\
\hline
\end{tabular}

$-83-$ 
Table 16--CHEMICAL-QUALITY SURVEYS OF SOMERVILLE LAKE MAY 21, 1980--Continued

301805096332501 SOMERVILLE LAKE SITE CC

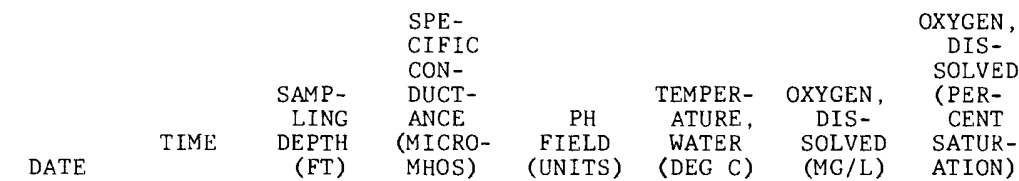

\begin{tabular}{|c|c|c|c|c|c|c|c|}
\hline MAY & & & & & & & \\
\hline $\begin{array}{l}21 \ldots \\
21 \ldots\end{array}$ & $\begin{array}{l}1341 \\
1343\end{array}$ & $\begin{array}{r}1.0 \\
14.0\end{array}$ & $\begin{array}{l}363 \\
363\end{array}$ & $\begin{array}{l}8.4 \\
7.0\end{array}$ & $\begin{array}{l}26.0 \\
23.5\end{array}$ & $\begin{array}{r}10.1 \\
6.2\end{array}$ & $\begin{array}{r}125 \\
73\end{array}$ \\
\hline
\end{tabular}

301847096334601 SOMERVILLE LAKE SITE DR

\begin{tabular}{|c|c|c|c|c|c|c|c|}
\hline DATE & TIME & $\begin{array}{l}\text { SAMP- } \\
\text { LING } \\
\text { DEPTH } \\
(\mathrm{FT})\end{array}$ & $\begin{array}{l}\text { SPE- } \\
\text { CIFIC } \\
\text { CON- } \\
\text { DUCT- } \\
\text { ANCE } \\
\text { (MICRO- } \\
\text { MHOS) }\end{array}$ & $\begin{array}{c}\text { PH } \\
\text { FIELD } \\
\text { (UNITS) }\end{array}$ & $\begin{array}{l}\text { TEMPER- } \\
\text { ATURE, } \\
\text { WATER } \\
\left(\begin{array}{ll}\text { DEG } & \text { C) }\end{array}\right.\end{array}$ & $\begin{array}{c}\text { OXYGEN, } \\
\text { DIS- } \\
\text { SOLVED } \\
(M G / L)\end{array}$ & $\begin{array}{c}\text { OXYGEN, } \\
\text { DIS- } \\
\text { SOLVED } \\
\text { (PER- } \\
\text { CENT } \\
\text { SATUR- } \\
\text { ATION) }\end{array}$ \\
\hline \multicolumn{8}{|l|}{ MAY } \\
\hline $\begin{array}{l}21 \ldots \\
21 \ldots \\
21 \ldots \\
21 \ldots\end{array}$ & $\begin{array}{l}1234 \\
1236 \\
1238 \\
1240\end{array}$ & $\begin{array}{r}1.0 \\
10.0 \\
20.0 \\
26.0\end{array}$ & $\begin{array}{l}368 \\
368 \\
368 \\
368\end{array}$ & $\begin{array}{l}8.4 \\
7.3 \\
6.8 \\
6.7\end{array}$ & $\begin{array}{l}26.0 \\
23.5 \\
22.5 \\
22.5\end{array}$ & $\begin{array}{l}9.9 \\
7.3 \\
5.2 \\
3.5\end{array}$ & $\begin{array}{r}122 \\
86 \\
60 \\
41\end{array}$ \\
\hline
\end{tabular}

301904096335601 SOMERVILLE I.AKE. SITE DC

\begin{tabular}{|c|c|c|c|c|c|c|c|c|c|c|c|c|}
\hline DATE & TIME & \multicolumn{2}{|c|}{$\begin{array}{l}\text { SPE- } \\
\text { CIFIC } \\
\text { CON- } \\
\text { DUCT- } \\
\text { ANCE } \\
\text { (MICRO- } \\
\text { MHOS) }\end{array}$} & \multicolumn{2}{|c|}{$\begin{array}{c}\text { PH } \\
\text { FIELD } \\
\text { (UNITS) }\end{array}$} & 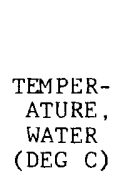 & \multicolumn{2}{|c|}{$\begin{array}{l}\text { TRANS- } \\
\text { PAR- } \\
\text { ENCY } \\
\text { (SECCHI } \\
\text { DISK) } \\
(M)\end{array}$} & \multicolumn{2}{|c|}{$\begin{array}{c}\text { OXYGEN, } \\
\text { DIS- } \\
\text { SOLVED } \\
(\mathrm{MG} / \mathrm{L})\end{array}$} & \multicolumn{2}{|c|}{$\begin{array}{c}\text { OXYGEN, } \\
\text { DIS- } \\
\text { SOLVED } \\
\text { (PER- } \\
\text { CENT } \\
\text { SATUR- } \\
\text { ATION) }\end{array}$} \\
\hline $\begin{array}{l}\text { MAY } \\
21 \ldots \\
21 \ldots \\
21 \ldots \\
21 \ldots\end{array}$ & $\begin{array}{l}1217 \\
1219 \\
1221 \\
1223\end{array}$ & $\begin{array}{l}1.0 \\
10.0 \\
20.0 \\
28.0\end{array}$ & $\begin{array}{l}354 \\
368 \\
368 \\
368\end{array}$ & & $\begin{array}{l}8.4 \\
7.2 \\
6.8 \\
6.7\end{array}$ & $\begin{array}{l}26.5 \\
23.5 \\
23.0 \\
22.5\end{array}$ & & $\begin{array}{c}.82 \\
-- \\
-- \\
--\end{array}$ & & $\begin{array}{l}9.7 \\
7.0 \\
4.6 \\
2.9\end{array}$ & & $\begin{array}{r}121 \\
82 \\
53 \\
34\end{array}$ \\
\hline $\begin{array}{l}\text { HARD- } \\
\text { NESS } \\
\text { (MG/L } \\
\text { AS } \\
\text { CACO3) }\end{array}$ & $\begin{array}{l}\text { HARD- } \\
\text { NESS } \\
\text { NONCAR- } \\
\text { BONATE } \\
\text { (MG/L } \\
\text { CACO3) }\end{array}$ & $\begin{array}{l}\text { CALCIUM } \\
\text { DIS- } \\
\text { SOLVED } \\
\text { (MG/L } \\
\text { AS CA) }\end{array}$ & $\begin{array}{r}\text { MAC } \\
\text { SI } \\
\text { DI } \\
\text { SOI } \\
\text { (MC } \\
\text { AS }\end{array}$ & $\begin{array}{l}\text { NE- } \\
\text { UM, } \\
\text { S- } \\
\text { UED } \\
/ L \\
\text { MG) }\end{array}$ & $\begin{array}{r}\text { SOD } \\
\text { DIS } \\
\text { SOL } \\
\text { (MC } \\
\text { AS }\end{array}$ & $\begin{array}{r}\text { SOI } \\
\text { SOI } \\
\text { TI } \\
\text { RAT }\end{array}$ & & $\begin{array}{r}\text { POT } \\
\text { S } \\
\text { D } \\
\text { SOL } \\
\text { (MC } \\
\text { AS }\end{array}$ & $\begin{array}{l}\text { AS- } \\
\text { UM, } \\
\text { S- } \\
\text { VED } \\
\text { / L } \\
\text { K) }\end{array}$ & $\begin{array}{r}\text { BICA } \\
\text { BONA } \\
\text { (MC } \\
A \\
H C C\end{array}$ & & $\begin{array}{c}\text { CAR- } \\
\text { BONATE } \\
(\mathrm{MG} / \mathrm{L} \\
\text { AS CO3) }\end{array}$ \\
\hline
\end{tabular}

\begin{tabular}{|c|c|c|c|c|c|c|c|c|c|}
\hline MAY & 99 & 58 & 28 & 70 & 25 & 11 & 5.6 & 50 & 0 \\
\hline $21 \ldots$ & $\ldots$ & - & - - & - - & - & - & -. & $=$ & - \\
\hline $21 \ldots$ & - - & - - & - - & -. & - & -- & -. & -- & -- \\
\hline $21 \ldots$ & 110 & 61 & 30 & 7.4 & 26 & 1.1 & 5.9 & 54 & 0 \\
\hline
\end{tabular}

\begin{tabular}{|c|c|c|c|c|c|c|c|c|}
\hline DATE & $\begin{array}{l}\text { SULFATE } \\
\text { DIS- } \\
\text { SOLVED } \\
\text { (MG/L } \\
\text { AS SO4) }\end{array}$ & $\begin{array}{l}\text { CHLO- } \\
\text { RIDE, } \\
\text { DIS- } \\
\text { SOLVED } \\
\text { (MG/L } \\
\text { AS CL) }\end{array}$ & $\begin{array}{l}\text { SILICA, } \\
\text { DIS- } \\
\text { SOLVED } \\
\text { (MG/L } \\
\text { AS } \\
\text { SIO2) }\end{array}$ & $\begin{array}{l}\text { SOLIDS, } \\
\text { SUM OF } \\
\text { CONSTI- } \\
\text { TUENTS, } \\
\text { DIS- } \\
\text { SOLVED } \\
\text { (MG/L) }\end{array}$ & $\begin{array}{c}\text { NITRO- } \\
\text { GEN, } \\
\text { NO2+NO3 } \\
\text { TOTAL } \\
\text { (MG/L } \\
\text { AS N) }\end{array}$ & $\begin{array}{l}\text { PHOS- } \\
\text { PHORUS, } \\
\text { TOTAL } \\
\text { (MG/L } \\
\text { AS P) }\end{array}$ & $\begin{array}{l}\text { IRON, } \\
\text { DIS- } \\
\text { SOLVED } \\
\text { (UG/L } \\
\text { AS FE) }\end{array}$ & $\begin{array}{l}\text { MANGA- } \\
\text { NESE, } \\
\text { DIS- } \\
\text { SOLVED } \\
\text { (UG/L } \\
\text { AS MN) }\end{array}$ \\
\hline \multicolumn{9}{|l|}{ MAY } \\
\hline $21 \ldots$ & 61 & 37 & 7.1 & 195 & .01 & .030 & 110 & 30 \\
\hline $21 \ldots$ & -- & -- & -- & -- & - & -- & -- & -- \\
\hline $21 \ldots$ & -- & -- & -- & -- & .02 & .040 & 190 & 50 \\
\hline $21 \ldots$ & 63 & 39 & 9.2 & 208 & .03 & .070 & 360 & 260 \\
\hline
\end{tabular}


301817096364101 SOMERVILLE LAKE SITE EC

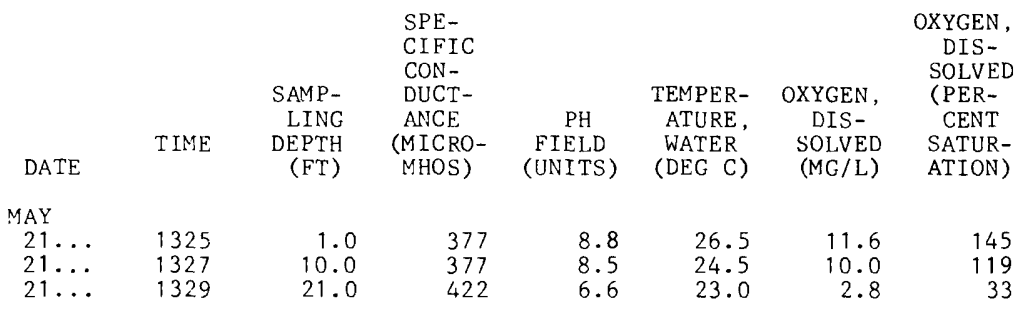

301754096380801 SOMERVILLE LAKE SITE FC

\begin{tabular}{|c|c|c|c|c|c|c|c|c|c|}
\hline DATE: & TIME & $\begin{array}{l}\text { SAMP- } \\
\text { LING } \\
\text { DEPTH } \\
\text { (FT) }\end{array}$ & $\begin{array}{l}\text { SPE- } \\
\text { CIFIC } \\
\text { CON- } \\
\text { DUCT- } \\
\text { ANCE } \\
\text { (MICRO- } \\
\text { MHOS) }\end{array}$ & $\begin{array}{c}\text { PH } \\
\text { FIELD } \\
\text { (UNITS) }\end{array}$ & $\begin{array}{c}\text { TEMPER- } \\
\text { ATURE, } \\
\text { WATER } \\
\text { (DEG C) }\end{array}$ & $\begin{array}{l}\text { TRANS- } \\
\text { PAR- } \\
\text { ENCY } \\
\text { (SECCHI } \\
\text { DISK) } \\
\text { (M) }\end{array}$ & $\begin{array}{c}\text { OXYGEN, } \\
\text { DIS- } \\
\text { SOLVED } \\
\text { (MG/L) }\end{array}$ & $\begin{array}{c}\text { OXYGEN, } \\
\text { DIS- } \\
\text { SOLVED } \\
\text { (PER- } \\
\text { CENT } \\
\text { SATUR- } \\
\text { ATION) }\end{array}$ & $\begin{array}{l}\text { HARD- } \\
\text { NESS } \\
(M G / L \\
\text { AS } \\
\text { CACO3) }\end{array}$ \\
\hline $\begin{array}{l}\text { MAY } \\
21 \ldots \\
21 \ldots \\
21 \ldots \\
21 \ldots\end{array}$ & $\begin{array}{l}1259 \\
1300 \\
1301 \\
1303\end{array}$ & $\begin{array}{r}1.0 \\
.4 \\
10.0 \\
14.0\end{array}$ & $\begin{array}{r}319 \\
-- \\
293 \\
293\end{array}$ & $\begin{array}{r}7.9 \\
- \\
6.6 \\
6.6\end{array}$ & $\begin{array}{r}27.5 \\
-- \\
25.0 \\
24.5\end{array}$ & $\begin{array}{c}.24 \\
-- \\
-- \\
--\end{array}$ & $\begin{array}{r}9.4 \\
-- \\
5.0 \\
4.8\end{array}$ & $\begin{array}{r}119 \\
-- \\
60 \\
57\end{array}$ & $\begin{array}{l}87 \\
-- \\
84\end{array}$ \\
\hline DATE: & $\begin{array}{l}\text { HARD- } \\
\text { NESS, } \\
\text { NONCAR- } \\
\text { BONATE } \\
\text { (MC/L } \\
\text { CACO3) }\end{array}$ & $\begin{array}{l}\text { CALCIUM } \\
\text { DIS- } \\
\text { SOLVED } \\
\text { (MG/L } \\
\text { AS CA) }\end{array}$ & $\begin{array}{l}\text { MAGNE- } \\
\text { SIUM, } \\
\text { DIS- } \\
\text { SOLVED } \\
\text { (MG/L } \\
\text { AS MG) }\end{array}$ & $\begin{array}{l}\text { SODIUM, } \\
\text { DIS- } \\
\text { SOLVED } \\
\text { (MG/L } \\
\text { AS NA) }\end{array}$ & $\begin{array}{c}\text { SODIUM } \\
\text { AD- } \\
\text { SORP- } \\
\text { TION } \\
\text { RATIO }\end{array}$ & $\begin{array}{l}\text { POTAS- } \\
\text { SIUM, } \\
\text { DIS- } \\
\text { SOLVED } \\
(\mathrm{MG} / \mathrm{L} \\
\text { AS K) }\end{array}$ & $\begin{array}{c}\text { BICAR- } \\
\text { BONATE } \\
\text { (MG / L } \\
\text { AS } \\
\text { HCO3) }\end{array}$ & $\begin{array}{c}\text { CAR- } \\
\text { BONATE } \\
\text { (MG/L } \\
\text { AS CO3) }\end{array}$ & $\begin{array}{l}\text { SULFATE } \\
\text { DIS- } \\
\text { SOLVED } \\
\text { (MG/L } \\
\text { AS SO4) }\end{array}$ \\
\hline
\end{tabular}

\begin{tabular}{|c|c|c|c|c|c|c|c|c|c|}
\hline MAY & & & & & & & & & \\
\hline $21 \ldots$ & 51 & 24 & 6.6 & 23 & 1.1 & 6.2 & 44 & 0 & 53 \\
\hline $21 \ldots$ & -- & -- & -- & -- & -- & -- & -- & -- & -- \\
\hline $21 \ldots$ & - & -- & -- & $1^{--}$ & $-\overline{-}$ & $-\overline{8}$ & $-\overline{-}$ & $-\overline{0}$ & $0^{--}$ \\
\hline & 46 & 23 & 6.4 & 21 & 1.0 & 6.8 & 46 & 0 & 49 \\
\hline
\end{tabular}

\begin{tabular}{|c|c|c|c|c|c|c|c|c|}
\hline DATF & $\begin{array}{l}\text { CHLO- } \\
\text { RIDE, } \\
\text { DIS- } \\
\text { SOLVED } \\
\text { (MG/L } \\
\text { AS CL) }\end{array}$ & $\begin{array}{l}\text { SILICA, } \\
\text { DIS- } \\
\text { SOLVED } \\
\text { (MG/L } \\
\text { AS } \\
\text { SIO2) }\end{array}$ & $\begin{array}{c}\text { SOLIDS, } \\
\text { SUM OF } \\
\text { CONSTI- } \\
\text { TUENTS, } \\
\text { DIS- } \\
\text { SOLVED } \\
\text { (MG/L) }\end{array}$ & $\begin{array}{c}\text { NITRO- } \\
\text { GEN, } \\
\text { NO2+NO3 } \\
\text { TOTAL } \\
\text { (MG/L } \\
\text { AS N) }\end{array}$ & $\begin{array}{l}\text { PHOS- } \\
\text { PHORUS, } \\
\text { TOTAL } \\
\text { (MG/L } \\
\text { AS P) }\end{array}$ & $\begin{array}{l}\text { IRON, } \\
\text { DIS- } \\
\text { SOLVED } \\
\text { (UG / L } \\
\text { AS FE) }\end{array}$ & $\begin{array}{l}\text { MANGA- } \\
\text { NESE, } \\
\text { DIS- } \\
\text { SOLVED } \\
\text { (UG/L } \\
\text { AS MN) }\end{array}$ & $\begin{array}{c}\text { PHYTO- } \\
\text { PLANK- } \\
\text { TON, } \\
\text { TOTAL } \\
\text { (CELLS } \\
\text { PER ML) }\end{array}$ \\
\hline \multicolumn{9}{|l|}{ MAY } \\
\hline $21 \ldots$ & 35 & 11 & 181 & .02 & .170 & 410 & 50 & -- \\
\hline $21 \ldots$ & -- & - & -- & - & -- & - & -- & 36000 \\
\hline & - & - & - & -- & -- & -- & -- & -- \\
\hline & 31 & 15 & 176 & .05 & .110 & 1100 & 220 & -- \\
\hline
\end{tabular}


$\mathrm{FT}=$ feet $;$ MICROMHOS $=$ micromhos per centimeter at $25^{\circ}$ Celsius; ${ }^{\circ} \mathrm{C}$ - degrees Celsius; $\mathrm{MG} / \mathrm{L}=\mathrm{milligrams}$ per liter; UG/L = micrograms per liter

301908096313101 SOMERVILLE LAKE SITE AC

\begin{tabular}{|c|c|c|c|c|c|c|c|c|c|}
\hline DATE & TIME & $\begin{array}{l}\text { SAMP- } \\
\text { LING } \\
\text { DEPTH } \\
(\mathrm{FT})\end{array}$ & $\begin{array}{l}\text { SPE- } \\
\text { CIFIC } \\
\text { CON- } \\
\text { DUCT- } \\
\text { ANCE } \\
\text { (MICRO- } \\
\text { MHOS) }\end{array}$ & $\begin{array}{c}\text { PH } \\
\text { FIELD } \\
\text { (UNITS) }\end{array}$ & $\begin{array}{l}\text { TEMPER- } \\
\text { ATURE, } \\
\text { WATER } \\
\text { (DEG C) }\end{array}$ & $\begin{array}{l}\text { TRANS- } \\
\text { PAR- } \\
\text { ENCY } \\
\text { (SECCHI } \\
\text { DISK) } \\
\text { (M) }\end{array}$ & $\begin{array}{c}\text { OXYGEN, } \\
\text { UIS- } \\
\text { SOLVED } \\
\text { (MG/L) }\end{array}$ & $\begin{array}{c}\text { OXYGEN, } \\
\text { DIS- } \\
\text { SOLVED } \\
\text { (PER- } \\
\text { CENT } \\
\text { SATUR- } \\
\text { ATION) }\end{array}$ & $\begin{array}{l}\text { HARD- } \\
\text { NESS } \\
(1 \mathrm{G} / \mathrm{L} \\
\mathrm{AS} \\
\mathrm{CACO} 3)\end{array}$ \\
\hline $\begin{array}{l}\text { AUG } \\
29 \ldots \\
29 \ldots \\
29 \ldots \\
29 \ldots\end{array}$ & $\begin{array}{l}0920 \\
0922 \\
0924 \\
0926\end{array}$ & $\begin{array}{r}1.0 \\
10.0 \\
20.0 \\
25.0\end{array}$ & $\begin{array}{l}428 \\
428 \\
428 \\
428\end{array}$ & $\begin{array}{l}7.1 \\
6.9 \\
6.9 \\
6.9\end{array}$ & $\begin{array}{l}28.5 \\
28.5 \\
28.5 \\
28.5\end{array}$ & $\begin{array}{c}61 \\
-- \\
-- \\
--\end{array}$ & $\begin{array}{l}5.5 \\
4.6 \\
4.6 \\
4.6\end{array}$ & $\begin{array}{l}70 \\
58 \\
58 \\
58\end{array}$ & $\begin{array}{c}110 \\
10 \\
110\end{array}$ \\
\hline DATE & $\begin{array}{l}\text { HARD- } \\
\text { NESS } \\
\text { NONCAR- } \\
\text { BONATE } \\
\text { (MG/L } \\
\text { CACO3) }\end{array}$ & $\begin{array}{l}\text { CALCIUM } \\
\text { DIS- } \\
\text { SOLVED } \\
\text { (MG/L } \\
\text { AS CA) }\end{array}$ & $\begin{array}{l}\text { MAGNE- } \\
\text { SIUM, } \\
\text { DIS- } \\
\text { SOLVED } \\
\text { (MC/L } \\
\text { AS MG) }\end{array}$ & $\begin{array}{l}\text { SODIUM, } \\
\text { DIS- } \\
\text { SOLVED } \\
\text { (MG/L } \\
\text { AS NA) }\end{array}$ & $\begin{array}{l}\text { SODIUM } \\
\text { AD- } \\
\text { SORP- } \\
\text { TION } \\
\text { RATIO }\end{array}$ & $\begin{array}{l}\text { POTAS- } \\
\text { SIUM, } \\
\text { DIS- } \\
\text { SOLVED } \\
(M G / L \\
\text { AS K) }\end{array}$ & $\begin{array}{c}\text { BICAR- } \\
\text { BONATE } \\
\text { (MG /L } \\
\text { AS } \\
\text { HCO3) }\end{array}$ & $\begin{array}{c}\text { CAR- } \\
\text { BONATE } \\
\text { (MG/L } \\
\text { AS CO3) }\end{array}$ & $\begin{array}{l}\text { SULFATE } \\
\text { DIS- } \\
\text { SOLVED } \\
\text { (NG/L } \\
\text { AS SO4) }\end{array}$ \\
\hline
\end{tabular}

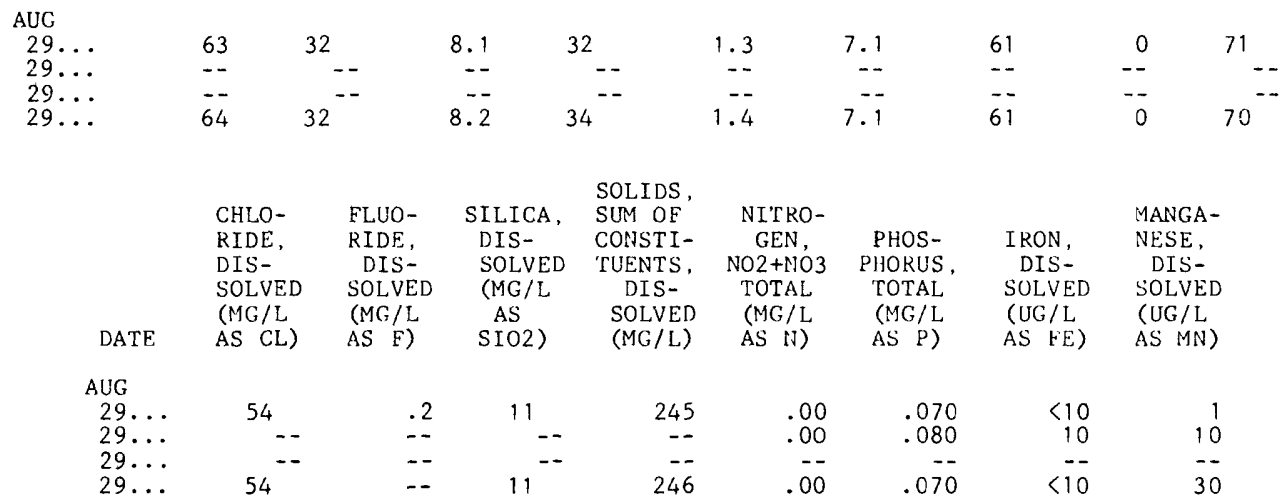

301940096315801 SOMERVILLE LAKE SITE AL

\begin{tabular}{|c|c|c|c|c|c|c|c|}
\hline & & & $\begin{array}{l}\text { SPE- } \\
\text { CIFIC } \\
\text { CON- }\end{array}$ & & & & $\begin{array}{c}\text { OXYGEN, } \\
\text { DIS- } \\
\text { SOLVED }\end{array}$ \\
\hline & & $\begin{array}{l}\text { SAMP- } \\
\text { LING }\end{array}$ & $\begin{array}{l}\text { DUCT- } \\
\text { ANCE }\end{array}$ & $\mathrm{PH}$ & $\begin{array}{l}\text { TEMPER- } \\
\text { ATURE, }\end{array}$ & $\begin{array}{l}\text { OXYGEN, } \\
\text { DIS- }\end{array}$ & $\begin{array}{l}\text { (PEK- } \\
\text { CENT }\end{array}$ \\
\hline ATE & TIME & $\begin{array}{c}\text { DEPTH } \\
\text { (FT) }\end{array}$ & $\begin{array}{l}\text { (MICRO- } \\
\text { MHOS) }\end{array}$ & $\begin{array}{l}\text { FIELDD } \\
\text { (UNI'TS) }\end{array}$ & $\begin{array}{l}\text { WATER } \\
\left(\begin{array}{ll}\text { DEG } & \text { C) }\end{array}\right.\end{array}$ & $\begin{array}{l}\text { SOLVED } \\
(M G / L)\end{array}$ & $\begin{array}{l}\text { SATUR- } \\
\text { ATIOIJ) }\end{array}$ \\
\hline
\end{tabular}

\begin{tabular}{|c|c|c|c|c|c|c|c|}
\hline \multicolumn{8}{|l|}{ AUG } \\
\hline $\begin{array}{l}29 \ldots \\
29 \ldots\end{array}$ & $\begin{array}{l}0945 \\
0947\end{array}$ & $\begin{array}{r}1.0 \\
10.0 \\
13.0\end{array}$ & $\begin{array}{l}428 \\
428\end{array}$ & $\begin{array}{l}7.4 \\
7.1 \\
7\end{array}$ & $\begin{array}{l}29.0 \\
28.5 \\
28.5\end{array}$ & $\begin{array}{l}6.8 \\
5.6 \\
5.6\end{array}$ & $\begin{array}{l}87 \\
71 \\
71\end{array}$ \\
\hline
\end{tabular}

302026096341501 SOMERVILLE LAKE SITE BC

\begin{tabular}{|c|c|c|c|c|c|c|c|}
\hline DATE & TIME & $\begin{array}{l}\text { SAMP- } \\
\text { LING } \\
\text { DEPTH } \\
(\mathrm{FT})\end{array}$ & $\begin{array}{l}\text { SPE- } \\
\text { CIFIC } \\
\text { CON- } \\
\text { DUCT- } \\
\text { ANCE } \\
\text { (MICRO- } \\
\text { MHOS) }\end{array}$ & $\begin{array}{c}\text { PH } \\
\text { FIELD } \\
\text { (UNITS) }\end{array}$ & $\begin{array}{c}\text { TEMPER- } \\
\text { ATURE , } \\
\text { WATER } \\
\text { (DEG C) }\end{array}$ & $\begin{array}{c}\text { OXYGEN, } \\
\text { DIS- } \\
\text { SOLVED } \\
\text { (MG/L) }\end{array}$ & $\begin{array}{c}\text { OXYGEN, } \\
\text { DIS- } \\
\text { SOLVED } \\
\text { (PER- } \\
\text { CENT } \\
\text { SATUR- } \\
\text { ATION) }\end{array}$ \\
\hline $\begin{array}{l}\text { AUG } \\
29 \ldots \\
29 \ldots\end{array}$ & $\begin{array}{l}1005 \\
1007\end{array}$ & $\begin{array}{l}1.0 \\
8.0\end{array}$ & $\begin{array}{l}432 \\
432\end{array}$ & $\begin{array}{l}7.9 \\
7.5\end{array}$ & $\begin{array}{l}30.0 \\
30.0\end{array}$ & $\begin{array}{l}6.8 \\
5.9\end{array}$ & $\begin{array}{l}88 \\
77\end{array}$ \\
\hline
\end{tabular}


Table 17--CHEMICAL-QUALITY SURVEYS OF SOMERVILLE LAKE AUGUST 29, 1980--Continued

301805096332501 SOMERVILLE LAKE SITE CC

\begin{tabular}{|c|c|c|c|c|c|c|c|}
\hline DATE & TIME & $\begin{array}{l}\text { SAMP- } \\
\text { LING } \\
\text { DEPTH } \\
\text { (FT) }\end{array}$ & $\begin{array}{l}\text { SPE- } \\
\text { CIFIC } \\
\text { CON- } \\
\text { DUCT- } \\
\text { ANCE } \\
\text { (MICRO- } \\
\text { MHOS) }\end{array}$ & $\begin{array}{c}\text { PH } \\
\text { FIELD } \\
\text { (UNITS) }\end{array}$ & $\begin{array}{l}\text { TEMPER- } \\
\text { ATURE, } \\
\text { WATER } \\
\text { (DEG C) }\end{array}$ & $\begin{array}{c}\text { OXYGEN, } \\
\text { DIS- } \\
\text { SOLVED } \\
(M G / L)\end{array}$ & $\begin{array}{c}\text { OXYGEN, } \\
\text { DIS- } \\
\text { SOLVED } \\
\text { (PER- } \\
\text { CENT } \\
\text { SATUR- } \\
\text { ATION) }\end{array}$ \\
\hline $\begin{array}{l}\text { AUGG } \\
29 \ldots \\
29 \ldots\end{array}$ & $\begin{array}{l}1215 \\
1217\end{array}$ & $\begin{array}{l}1.0 \\
8.0\end{array}$ & $\begin{array}{l}432 \\
432\end{array}$ & $\begin{array}{l}7.5 \\
6.8\end{array}$ & $\begin{array}{l}29.5 \\
28.5\end{array}$ & $\begin{array}{l}6.6 \\
3.2\end{array}$ & $\begin{array}{l}85 \\
41\end{array}$ \\
\hline
\end{tabular}

301847096334601 SOMERVILLE, LAAKE SITE DR

\begin{tabular}{|c|c|c|c|c|c|c|c|}
\hline DATE & TIME & $\begin{array}{r}\text { SAMP- } \\
\text { LING } \\
\text { DEPTH } \\
\text { (FT) }\end{array}$ & $\begin{array}{l}\text { SPE- } \\
\text { CIFIC } \\
\text { CON- } \\
\text { DUCT- } \\
\text { ANCE } \\
\text { (MICRO- } \\
\text { MHOS) }\end{array}$ & $\begin{array}{c}\text { PH } \\
\text { FIELD } \\
\text { (UNI'S) }\end{array}$ & $\begin{array}{c}\text { TEMPER- } \\
\text { ATURE, } \\
\text { WATER } \\
\text { (DEG C) }\end{array}$ & $\begin{array}{c}\text { OXYGEN, } \\
\text { DIS- } \\
\text { SOLVED } \\
(M G / L)\end{array}$ & $\begin{array}{c}\text { OXYGEN } \\
\text { DIS- } \\
\text { SOLVED } \\
\text { (PER- } \\
\text { CENT } \\
\text { SATUR- } \\
\text { ATION) }\end{array}$ \\
\hline & & & & & & & \\
\hline $\begin{array}{l}29 \ldots \\
29 \ldots \\
29 \ldots\end{array}$ & $\begin{array}{l}1045 \\
1047 \\
1049\end{array}$ & $\begin{array}{r}1.0 \\
10.0 \\
19.0\end{array}$ & $\begin{array}{l}432 \\
432 \\
432\end{array}$ & $\begin{array}{l}7.7 \\
6.9 \\
6.8\end{array}$ & $\begin{array}{l}29.5 \\
29.0 \\
29.0\end{array}$ & $\begin{array}{l}6.9 \\
4.0 \\
2.6\end{array}$ & 88 \\
\hline
\end{tabular}

301904096335601 SOMERVILLE, LAKE SITE DC

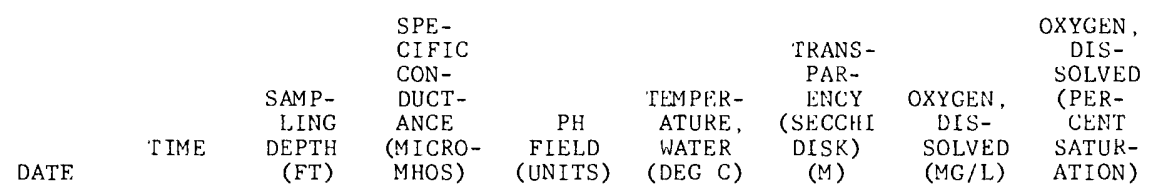

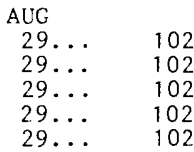

MHOS) (UNITS)

$\begin{array}{lll} & (M) & (M G / L)\end{array}$

ION)

\begin{tabular}{|c|c|c|c|c|c|c|c|c|c|}
\hline \multicolumn{10}{|c|}{ AUG } \\
\hline & $\begin{array}{l}29 \ldots \\
29 \ldots \\
29 \ldots \\
29 \ldots \\
29 \ldots\end{array}$ & $\begin{array}{l}1020 \\
1022 \\
1024 \\
1026 \\
1028\end{array}$ & $\begin{array}{r}1.0 \\
5.0 \\
10.0 \\
20.0 \\
25.0\end{array}$ & $\begin{array}{l}432 \\
432 \\
432 \\
432 \\
432\end{array}$ & $\begin{array}{l}7.8 \\
7.4 \\
7.2 . \\
7.0 \\
7.0\end{array}$ & $\begin{array}{l}29.5 \\
29.0 \\
29.0 \\
29.0 \\
29.0\end{array}$ & $\begin{array}{c}61 \\
-- \\
-- \\
-- \\
--\end{array}$ & $\begin{array}{l}6.8 \\
5.8 \\
5.3 \\
4.6 \\
4.6\end{array}$ & $\begin{array}{l}87 \\
74 \\
68 \\
59 \\
59\end{array}$ \\
\hline DATE & $\begin{array}{l}\text { HARD- } \\
\text { NESS } \\
\text { (MG/L } \\
\text { AS } \\
\text { CACO3) }\end{array}$ & $\begin{array}{l}\text { HARD- } \\
\text { NESS } \\
\text { NONCAR- } \\
\text { BONATE } \\
(\mathrm{MG} / \mathrm{L} \\
\text { CACO3) }\end{array}$ & $\begin{array}{l}\text { CALC IUM } \\
\text { DIS- } \\
\text { SOLVED } \\
\text { (MG/L } \\
\text { AS CA) }\end{array}$ & $\begin{array}{l}\text { MAGNE- } \\
\text { SIUM, } \\
\text { DIS- } \\
\text { SOLVED } \\
\text { (MG/L } \\
\text { AS MG) }\end{array}$ & $\begin{array}{l}\text { SODIUM, } \\
\text { DIS- } \\
\text { SOLVED } \\
\text { (MG/L } \\
\text { AS NA) }\end{array}$ & $\begin{array}{c}\text { SODIUM } \\
\text { AD- } \\
\text { SORP- } \\
\text { TION } \\
\text { RAIIO }\end{array}$ & $\begin{array}{l}\text { POTAS- } \\
\text { SIUM, } \\
\text { DIS- } \\
\text { SOLVED } \\
(M G / L \\
\text { AS K) }\end{array}$ & $\begin{array}{c}\text { BICAK- } \\
\text { BONATE } \\
(\mathrm{MG} / \mathrm{L} \\
\text { AS } \\
\mathrm{HCO} 3)\end{array}$ & $\begin{array}{c}\text { CAR- } \\
\text { BONATE } \\
(M C / L \\
\text { AS CO3) }\end{array}$ \\
\hline
\end{tabular}

\begin{tabular}{|c|c|c|c|c|c|c|c|c|c|}
\hline \multicolumn{10}{|l|}{ AUC } \\
\hline $29 \ldots$ & 120 & 72 & 35 & 8.6 & 33 & 1.3 & 7.1 & 62 & 0 \\
\hline $29 \ldots$ & -- & $\cdots$ & -- & -- & -- & -- & -- & -- & -- \\
\hline $29 \ldots$ & -- & -- & -- & -- & -- & -- & -- & -- & -- \\
\hline $29 \ldots$ & -- & $=-$ & -- & -- & -- & -- & -- & - & -- \\
\hline $29 \ldots$ & 120 & 65 & 33 & 8.2 & 33 & 1.3 & 7.1 & 62 & 0 \\
\hline
\end{tabular}

\begin{tabular}{|c|c|c|c|c|c|c|c|c|}
\hline DATE & $\begin{array}{l}\text { SULFATE } \\
\text { DIS- } \\
\text { SOLVED } \\
\text { (MG/L } \\
\text { AS SO4) }\end{array}$ & $\begin{array}{l}\text { CHLO- } \\
\text { RIDE, } \\
\text { DIS- } \\
\text { SOLVED } \\
\text { (MG/L } \\
\text { AS CL) }\end{array}$ & $\begin{array}{l}\text { SILICA, } \\
\text { DIS- } \\
\text { SOLVED } \\
\text { (MG/L } \\
\text { AS } \\
\text { SIO2) }\end{array}$ & $\begin{array}{l}\text { SOLIDS, } \\
\text { SUM OF } \\
\text { CONSTI- } \\
\text { TUENTS, } \\
\text { DIS- } \\
\text { SOLVED } \\
\text { (MG/L) }\end{array}$ & $\begin{array}{c}\text { NITRO- } \\
\text { GEN, } \\
\text { NO2+NO3 } \\
\text { TOTAL } \\
(\mathrm{MC} / \mathrm{L} \\
\text { AS N) }\end{array}$ & $\begin{array}{c}\text { PHOS- } \\
\text { PHORUS, } \\
\text { TOTAL } \\
\text { (MG/L } \\
\text { AS P) }\end{array}$ & $\begin{array}{l}\text { IRON, } \\
\text { DIS- } \\
\text { SOLVED } \\
\text { (UG/L } \\
\text { AS FE) }\end{array}$ & $\begin{array}{l}\text { MANGA- } \\
\text { NESE, } \\
\text { DIS- } \\
\text { SOLVED } \\
\text { (UG/L } \\
\text { AS MN) }\end{array}$ \\
\hline \multicolumn{9}{|l|}{ AUG } \\
\hline $29 \ldots$ & 73 & 57 & 11 & 255 & .00 & .070 & $<10$ & 3 \\
\hline $29 \ldots$ & -- & - & -- & -- & -- & -- & -- & -- \\
\hline $29 \ldots$ & -- & -- & -- & -- & .00 & .080 & 10 & 10 \\
\hline $29 \ldots$ & -- & $=-$ & -- & -- & -- & - & -- & -- \\
\hline $29 \ldots$ & 70 & 54 & 11 & 247 & .00 & .090 & $<10$ & 80 \\
\hline
\end{tabular}


Table 17--CHEMICAL-QUALITY SURVEYS OF SOMERVILLE LAKE AUGUST 29, 1980--Continued

301817096364101 SOMERVILLE LAKE SITE EC

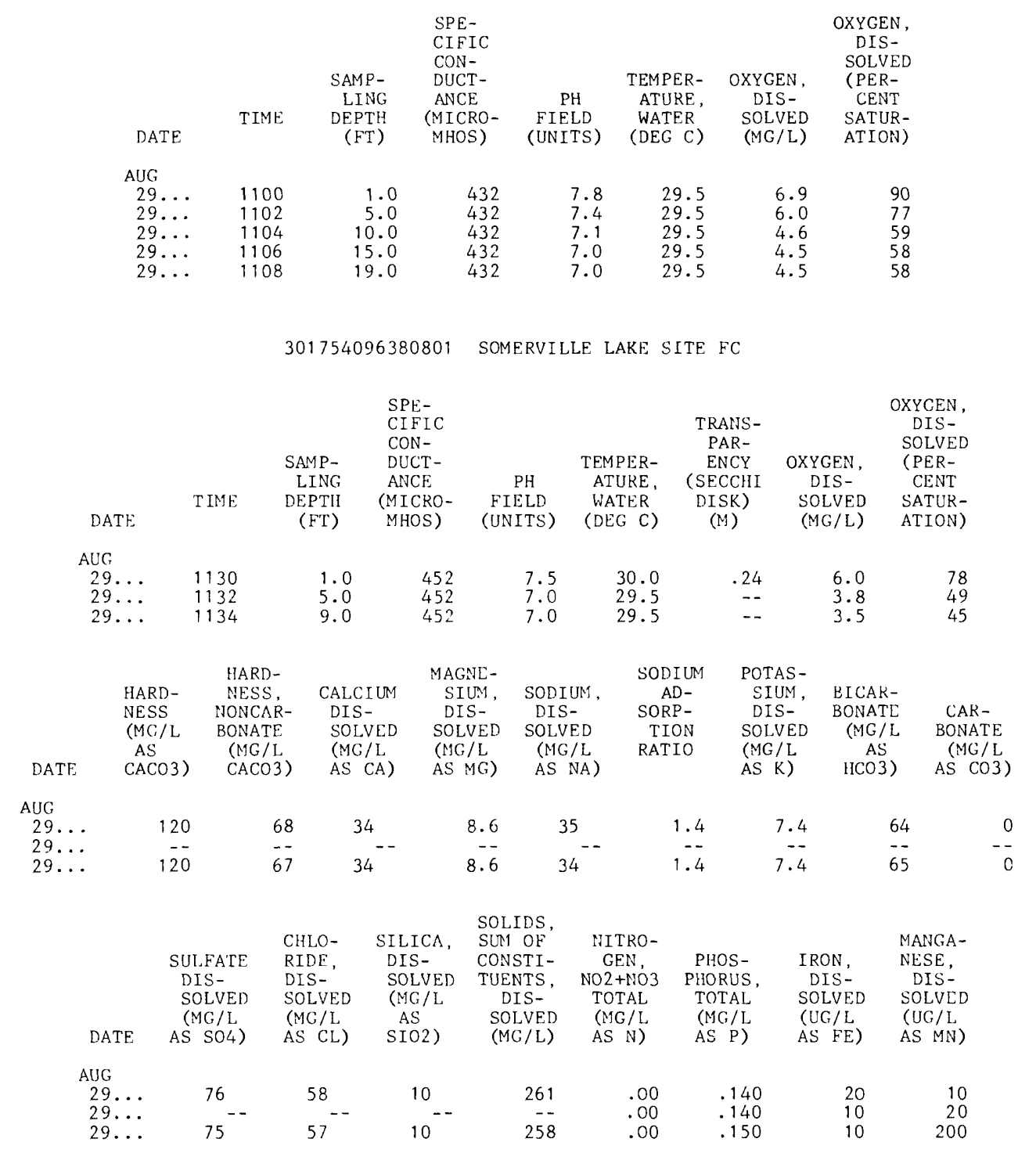

